Supporting Information:

\title{
Simple Open-Chain Phosphite-Olefin as Ligand for Rh-Catalyzed Asymmetric Arylation of Cyclic Ketimines: Efficient Enantioselective Access to gem-Diaryl $\alpha$-Amino Acid Derivatives
}

\author{
$\mathrm{Yi} \mathrm{Li}^{\dagger}$, Yue-Na $\mathrm{Yu}^{\dagger}$ and Ming-Hua $\mathrm{Xu}^{*}$ \\ ( ${ }^{\dagger}$ These authors contributed equally)
}

State Key Laboratory of Drug Research, Shanghai Institute of Materia Medica, Chinese Academy of Sciences, 555 Zuchongzhi Road, Shanghai 201203

E-mail:xumh@simm.ac.cn

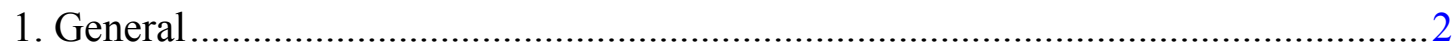

2. General procedure for the synthesis of $\mathbf{L 1 1}$ and characterization data of $\mathbf{L 1 1} \ldots \ldots \ldots . .2$

3. Copies of ${ }^{1} \mathrm{H},{ }^{13} \mathrm{C}$ NMR and ${ }^{31} \mathrm{P}$ NMR for $\mathbf{L 1 1}$ and $\mathrm{Rh} / \mathbf{L} 11$ complex. .....................

4. General procedures for $\mathrm{Rh}$-catalyzed 1,2-addition to cyclic imeines $\mathbf{1}$................... 8

5. General procedures for $\mathrm{Rh}$-catalyzed 1,2-addition to cyclic imeines 4 .................. 8

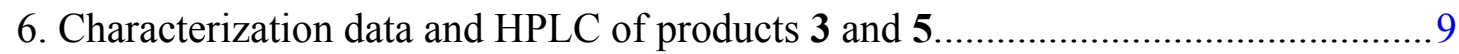

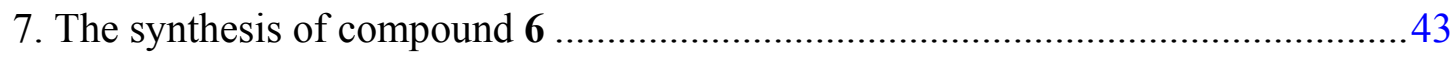

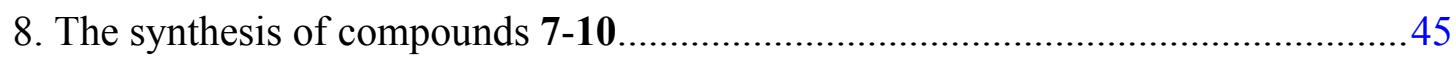

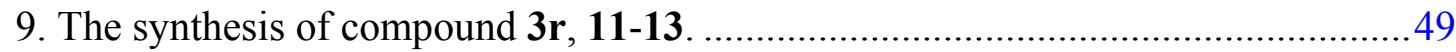

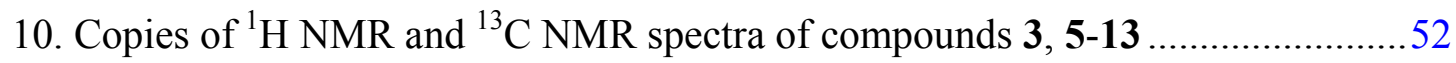




\section{General}

All reactions were carried out in flame-dried glassware with magnetic stirring under an atmosphere of argon. Solvents were dried and distilled by standard procedures. NMR spectra were recorded on a Varian spectrometer $\left(300 \mathrm{MHz}\right.$ for ${ }^{1} \mathrm{H}, 100 \mathrm{MHz}, 125 \mathrm{MHz}$ for ${ }^{13} \mathrm{C}, 200 \mathrm{MHz}$ for $\left.{ }^{31} \mathrm{P}\right)$. Chemical shifts are reported in $\delta$ ppm referenced to an internal $\mathrm{SiMe}_{4}$ standard for ${ }^{1} \mathrm{H} \mathrm{NMR}$ and chloroform- $d(\delta 77.16)$ for ${ }^{13} \mathrm{C}$ NMR. HPLC was performed on a JASCO 2000 instrument by using Daicel column with hexane/i-propanol as the eluent. High Resolution Mass Spectra (HRMS) were recorded on an Orbitrap mass spectrometer with ESI resource or Magnetic Sector for EI.

\section{General procedure for the synthesis of L11 and characterization data of L11.}

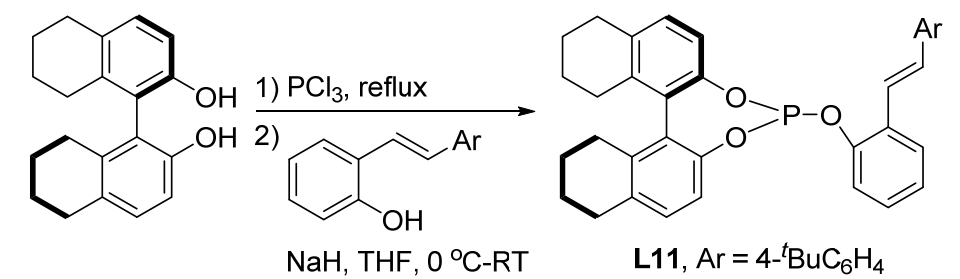

A solution of $(R)-\mathrm{H}_{8}$ - $\mathrm{BINOL}$ ( 1 equiv) and $\mathrm{PCl}_{3}$ (5 equiv) was stirred at $70{ }^{\circ} \mathrm{C}$ under argon overnight. The mixture was concentrated on a rotary evaporator and gave corresponding $(R)-\mathrm{H}_{8}$-BINOL-PCl. To a suspension of sodium hydride $(1.20 \mathrm{mmol}, 1.2$ equiv) in THF, (E)-2-(4-(tert-butyl)styryl)phenol $\left(1.20 \mathrm{mmol}, 1.2\right.$ equiv) was added at $0{ }^{\circ} \mathrm{C}$ under argon. After stirring for 30 minutes, freshly prepared $(R)-\mathrm{H}_{8} \mathrm{BINOL}-\mathrm{PCl}(1.0 \mathrm{mmol}, 1.0$ equiv) in $2 \mathrm{~mL}$ dry THF was added to the mixture. Subsequently, the mixture was allowed to come to RT and stirred for $3 \mathrm{~h}$. The solvent was removed under vacuum and the residue was purified by silica gel column chromatography to afford the corresponding ligand $\mathbf{L 1 1}$ as white foam $355 \mathrm{mg}, 62 \%$ yield, ${ }^{1} \mathrm{H}$ NMR $\left(300 \mathrm{MHz}, \mathrm{CDCl}_{3}\right) \delta 7.59(\mathrm{~d}, J=6.0 \mathrm{~Hz}, 1 \mathrm{H}), 7.39-7.25(\mathrm{~m}, 5 \mathrm{H}), 7.18-7.14(\mathrm{~m}, 2 \mathrm{H})$, 6.97-7.11 (m, 4H), $6.87(\mathrm{~s}, 2 \mathrm{H}), 2.77-2.59(\mathrm{~m}, 6 \mathrm{H}), 2.29-2.20(\mathrm{~m}, 2 \mathrm{H}), 1.92-1.68(\mathrm{~m}, 6 \mathrm{H})$, 1.56-1.52 (m, 2H), $1.28(\mathrm{~s}, 9 \mathrm{H}) ;{ }^{13} \mathrm{C}$ NMR $\left(125 \mathrm{MHz}, \mathrm{CDCl}_{3}\right) \delta 150.9,149.6\left(\mathrm{~d}, J_{\mathrm{CP}}=8.3 \mathrm{~Hz}\right)$, $146.1,145.8\left(\mathrm{~d}, J_{\mathrm{CP}}=3.8 \mathrm{~Hz}\right), 138.7,137.8,135.2,135.0,134.3,129.9,129.8\left(\mathrm{~d}, J_{\mathrm{CP}}=3.8 \mathrm{~Hz}\right)$, $129.61,129.56,129.48,128.4,127.9\left(\mathrm{~d}, J_{\mathrm{CP}}=2.5 \mathrm{~Hz}\right), 126.7,126.5,125.7,124.4,122.4,120.5(\mathrm{~d}$, $\left.J_{\mathrm{CP}}=13.6 \mathrm{~Hz}\right), 119.1,119.0,34.8,31.5,29.3,28.0,27.9,22.84,22.76,22.7,22.6 ;{ }^{31} \mathrm{P} \mathrm{NMR}(200$ $\mathrm{MHz}, \mathrm{CDCl}_{3}$ ) $\delta$ 138.0; HRMS (EI) for $\mathrm{C}_{38} \mathrm{H}_{39} \mathrm{O}_{3} \mathrm{P}[\mathrm{M}]^{+}$calcd 574.2637, found 574.2634. 
3. Copies of ${ }^{1} \mathrm{H},{ }^{13} \mathrm{C}$ NMR and ${ }^{31} \mathrm{P}$ NMR for $\mathrm{L} 11$ and Rh/L11 complex.

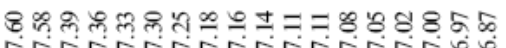

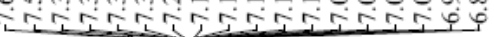

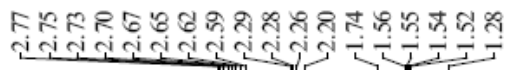

$\stackrel{8}{i}$
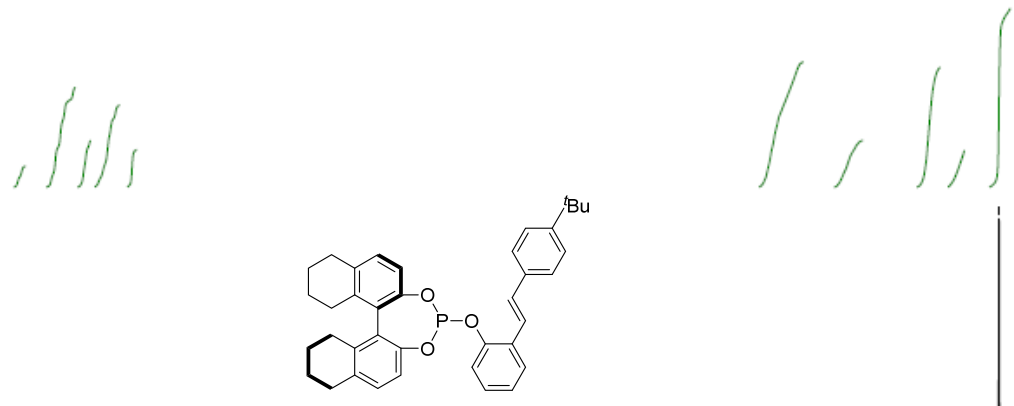

L11
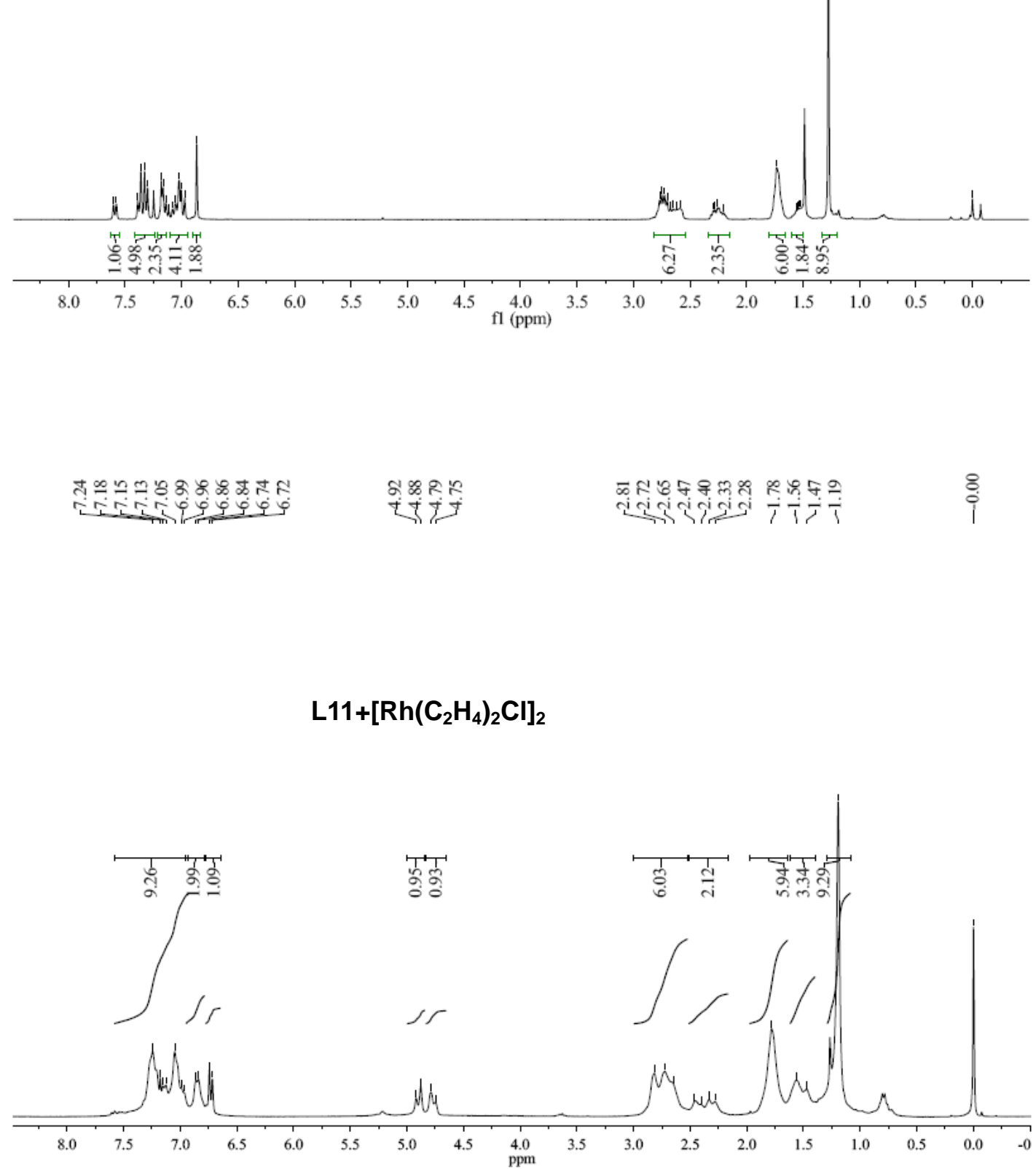


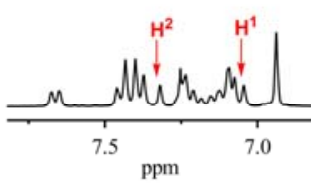

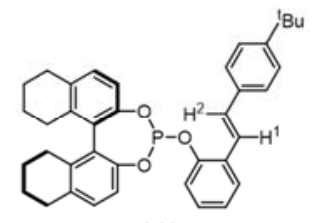

L11

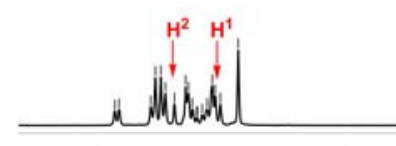

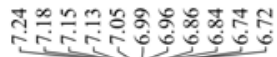

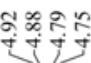
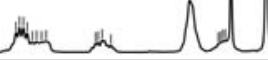

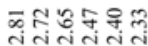

舟年的

$\mathrm{L} 11+\left[\mathrm{Rh}\left(\mathrm{C}_{2} \mathrm{H}_{4}\right)_{2} \mathrm{Cl}\right]_{2}$
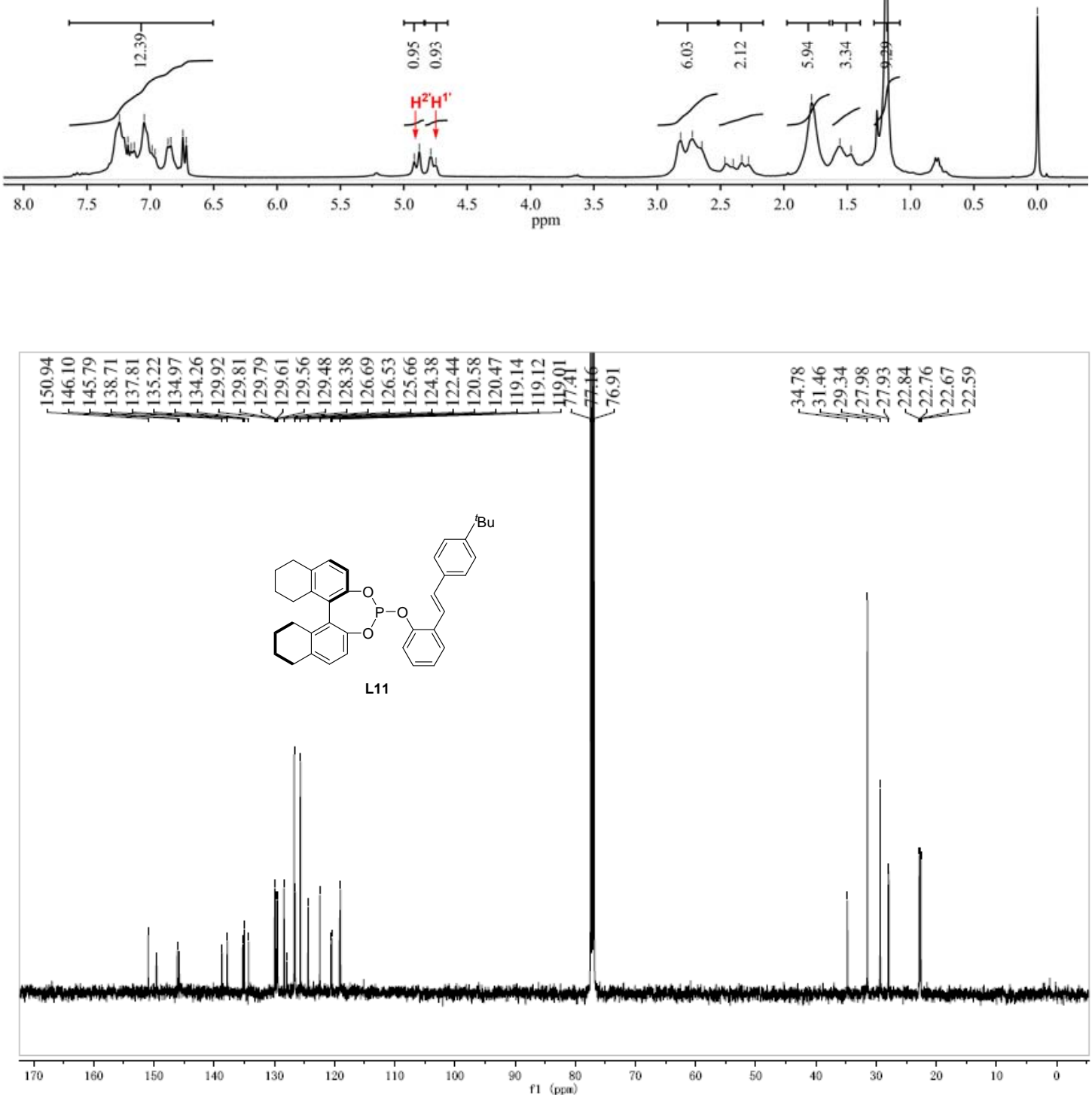

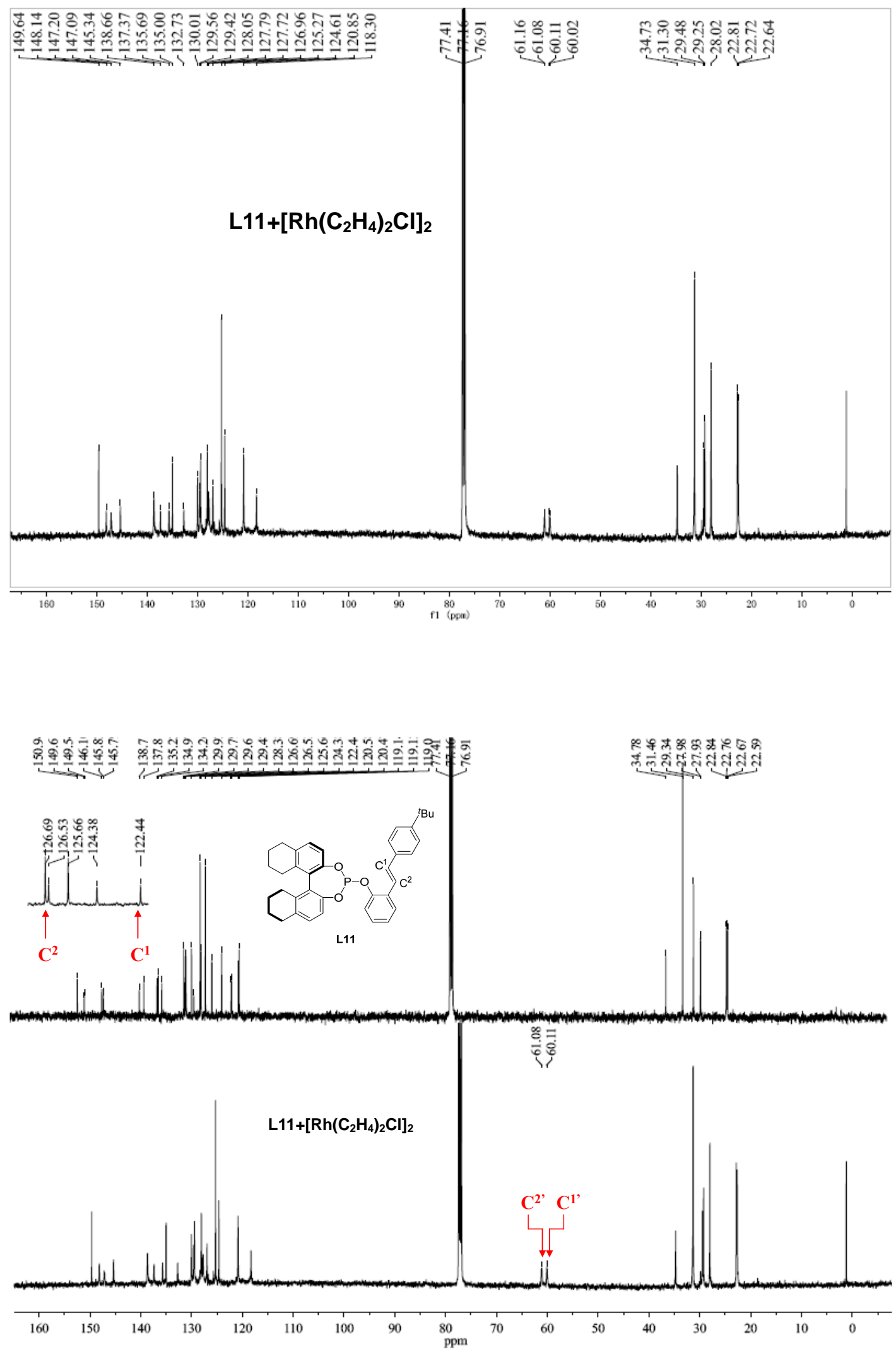
$\underset{\substack{0 \\ \frac{1}{1}}}{1}$

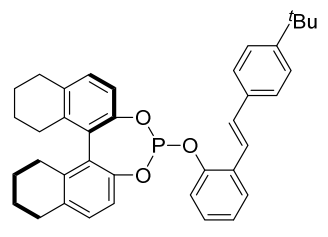

L11

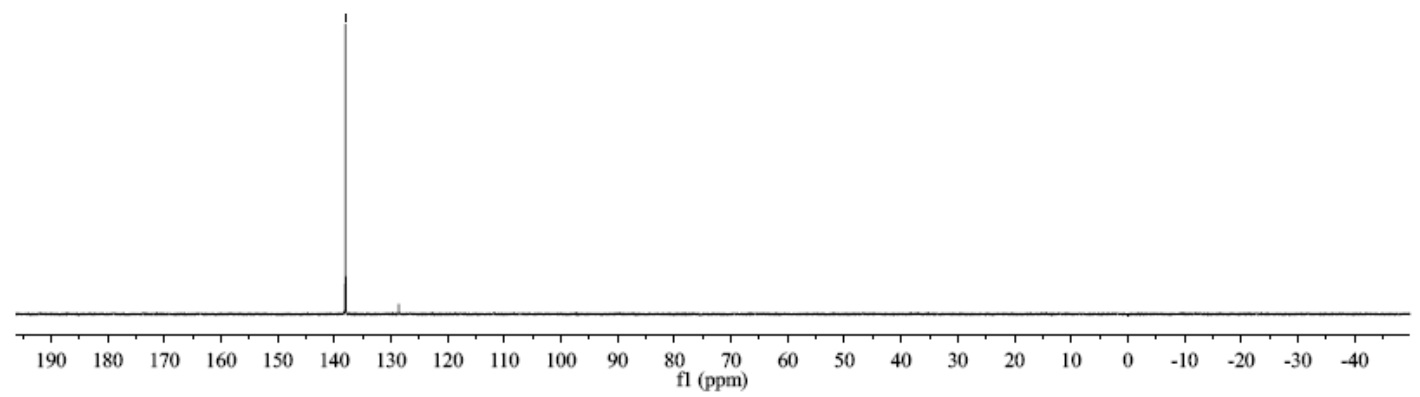

$\infty \overline{0}$
$\frac{9}{1}$

\section{$\operatorname{L11}+\left[\mathrm{Rh}\left(\mathrm{C}_{2} \mathrm{H}_{4}\right)_{2} \mathrm{Cl}\right]_{2}$}

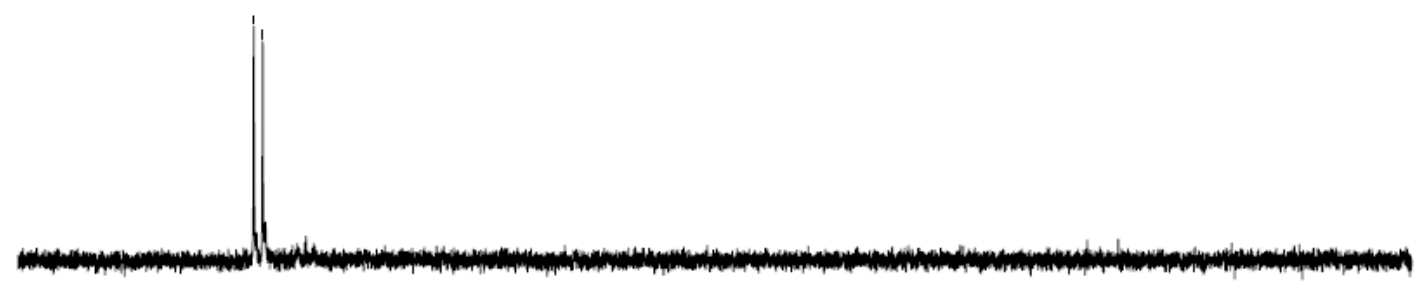

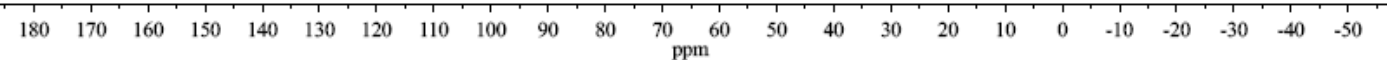




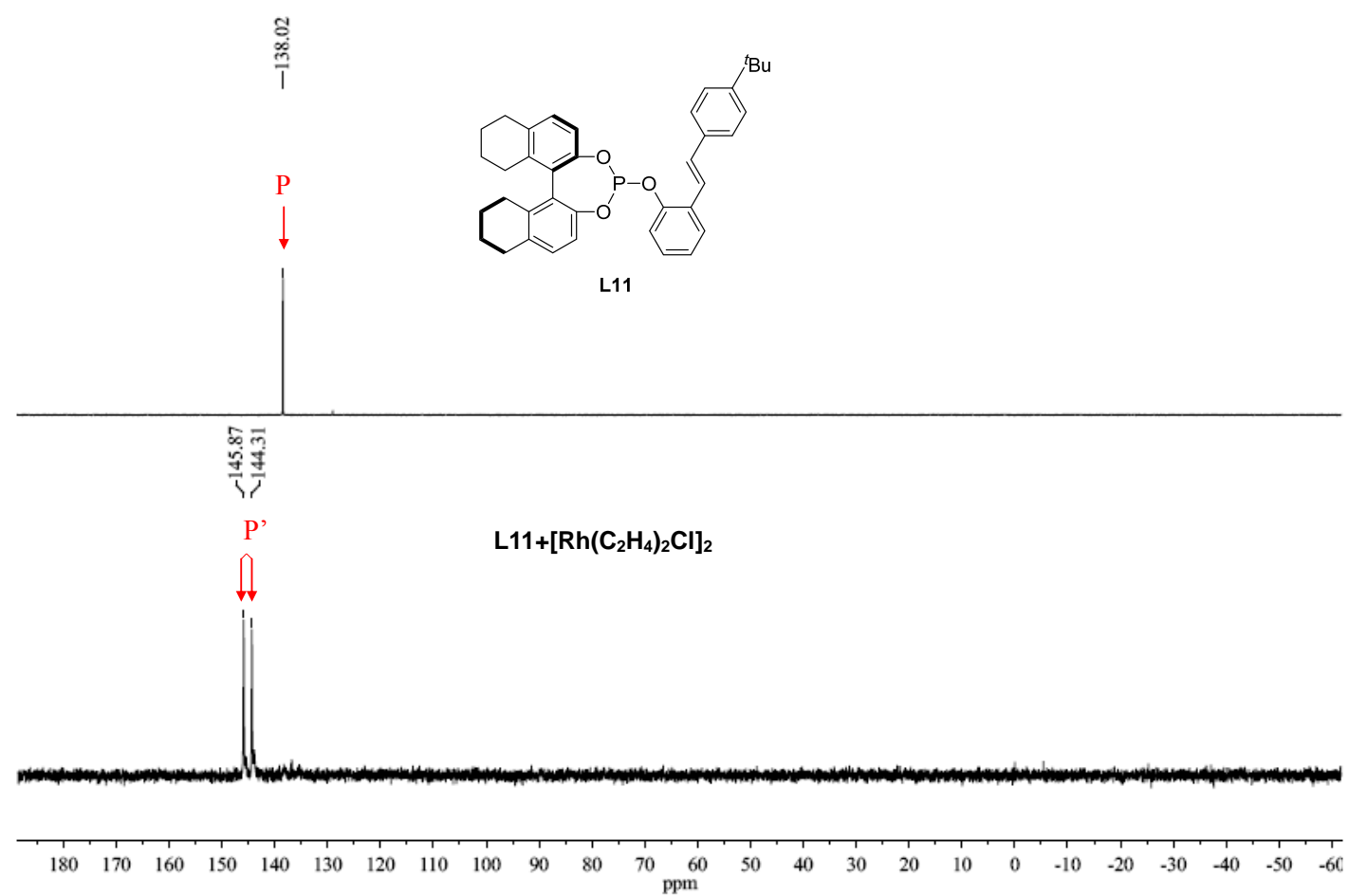




\section{General procedures for Rh-catalyzed 1,2-addition to cyclic imeines 1 .}

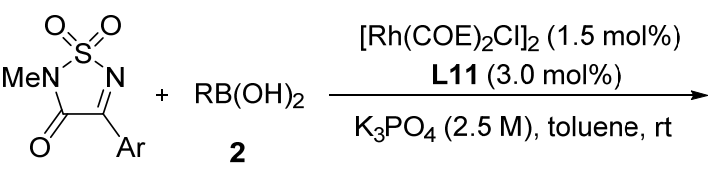

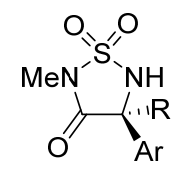

3

Under an argon atmosphere, a solution of cyclic imines $1(0.25 \mathrm{mmol}),\left[\mathrm{Rh}(\mathrm{COE})_{2} \mathrm{Cl}\right]_{2}(1.5 \mathrm{~mol} \%$, $2.7 \mathrm{mg}, 0.0075 \mathrm{mmol}$ of Rh), ligand $\mathbf{L 1 1}$ (3 $\mathrm{mol} \%, 4.3 \mathrm{mg}, 0.0075 \mathrm{mmol})$, and boronic acid 2 (0.5 $\mathrm{mmol}$ ) in $1.0 \mathrm{~mL}$ of toluene was stirred at room temperature for $30 \mathrm{~min}$. To this mixture was added aqueous $\mathrm{K}_{3} \mathrm{PO}_{4}(100 \mu \mathrm{L}, 2.5 \mathrm{M}, 0.25 \mathrm{mmol})$. After being stirred at room temperature for 3-12h (3 hours for 3a, 3c, 3o', 3p'; 6 hours for 3h, 3i, 3l-n, 3a', 3b', 3d'; 12 hours for 3b, 3d, 3e-g, 3j, 3k, 30-q), the mixture was concentrated under reduced pressure. The residue was purified by silica gel column chromatography to afford the corresponding product 3 .

\section{General procedures for Rh-catalyzed 1,2-addition to cyclic imeines 4 .}

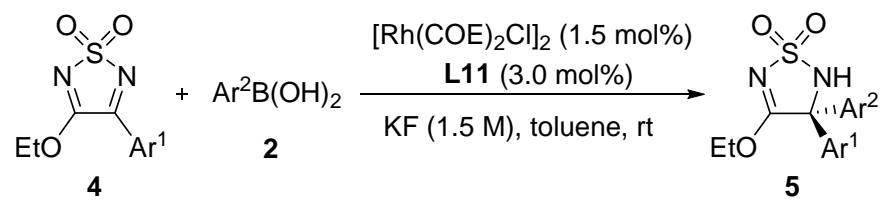

Under an argon atmosphere, a solution of cyclic imines $4(0.25 \mathrm{mmol}),\left[\mathrm{Rh}(\mathrm{COE})_{2} \mathrm{Cl}\right]_{2}(1.5 \mathrm{~mol} \%$, $2.7 \mathrm{mg}, 0.0075 \mathrm{mmol}$ of Rh), ligand $\mathbf{L 1 1}$ (3 $\mathrm{mol} \%, 4.3 \mathrm{mg}, 0.0075 \mathrm{mmol}$ ), and arylboronic acid 2 $(0.5 \mathrm{mmol})$ in $1.0 \mathrm{~mL}$ of toluene was stirred at room temperature for $30 \mathrm{~min}$. To this mixture was added aqueous KF (167 $\mu \mathrm{L}, 1.5 \mathrm{M}, 0.25 \mathrm{mmol})$. After being stirred at room temperature for 3-12h ( 3 hours for $\mathbf{5 a}, \mathbf{5 c}, \mathbf{5 h}, \mathbf{5 i}, \mathbf{5 k} ; 6$ hours for $\mathbf{5 d}, \mathbf{5 f}, \mathbf{5 g} ; 12$ hours for $\mathbf{5 b}, \mathbf{5 e}, \mathbf{5 j}, \mathbf{5 l}$ ), the mixture was concentrated under reduced pressure. The residue was purified by silica gel column chromatography to afford the corresponding product $\mathbf{5}$. 


\section{Characterization data and HPLC of products 3 and 5.}

(R)-2-methyl-4-phenyl-4-(p-tolyl)-1,2,5-thiadiazolidin-3-one 1,1-dioxide (3a)

White solid, $99 \%$ yield, $96 \%$ ee; $\mathrm{mp} 108-110{ }^{\circ} \mathrm{C}$.<smiles>Cc1ccc([C@]2(c3ccccc3)NS(=O)(=O)NC2=O)cc1</smiles>

$3 a$

$[\alpha]_{\mathrm{D}}{ }^{25}=+15.2\left(c 0.49, \mathrm{CHCl}_{3}\right) ;{ }^{1} \mathrm{H}$ NMR $\left(300 \mathrm{MHz}, \mathrm{CDCl}_{3}\right) \delta 7.47-7.40$ (m, 2H), $7.37-7.31(\mathrm{~m}, 3 \mathrm{H}), 7.28(\mathrm{~d}, J=8.3 \mathrm{~Hz}, 2 \mathrm{H}), 7.14(\mathrm{~d}, J=8.4 \mathrm{~Hz}$, 2H), $5.40(\mathrm{~s}, 1 \mathrm{H}), 3.11(\mathrm{~s}, 3 \mathrm{H}), 2.33(\mathrm{~s}, 3 \mathrm{H}) ;{ }^{13} \mathrm{C} \mathrm{NMR}\left(125 \mathrm{MHz}, \mathrm{CDCl}_{3}\right)$ $\delta 168.8,139.3,138.2,135.4,129.6,129.2,128.8,127.7,127.6,75.4,26.2$, 21.2; IR (KBr) v 3185, 1709, 1461, 1405, 1332, 1180, 1071, $971 \mathrm{~cm}^{-1}$; HRMS (ESI) for $\mathrm{C}_{16} \mathrm{H}_{15} \mathrm{O}_{3} \mathrm{~N}_{2} \mathrm{~S}$ [M-H] calcd 315.0809, found 315.0816.

HPLC ( $N$-Me derivative): Chiralpak IC column $(250 \mathrm{~mm})$; detected at $220 \mathrm{~nm}$; hexane/ $i$-propanol =97/3; flow $=0.7 \mathrm{~mL} / \mathrm{min}$; Retention time: $25.1 \mathrm{~min}$ (major), $28.6 \mathrm{~min}$.

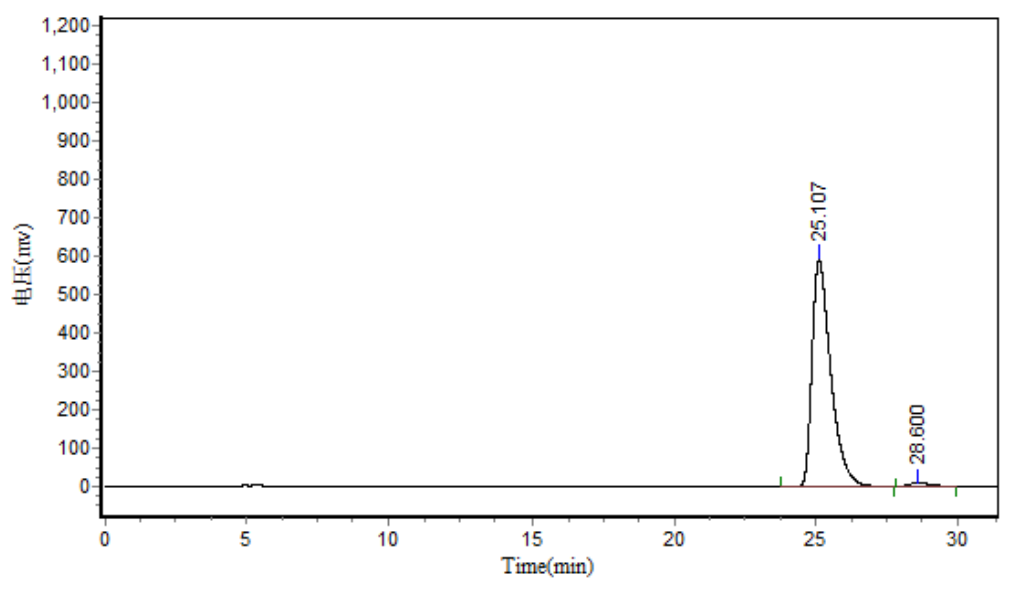

\begin{tabular}{|c|c|c|c|c|c|}
\hline \multicolumn{6}{|c|}{ Results } \\
\hline Peak No. & Peak ID & Ret Time & Height & Area & Conc. \\
\hline 1 & & 25.107 & 587456.563 & 27034008.000 & 98.1924 \\
\hline 2 & & 28.600 & 10331.174 & 497655.063 & 1.8076 \\
\hline Total & & & 597787.736 & 27531663.063 & 100.0000 \\
\hline
\end{tabular}

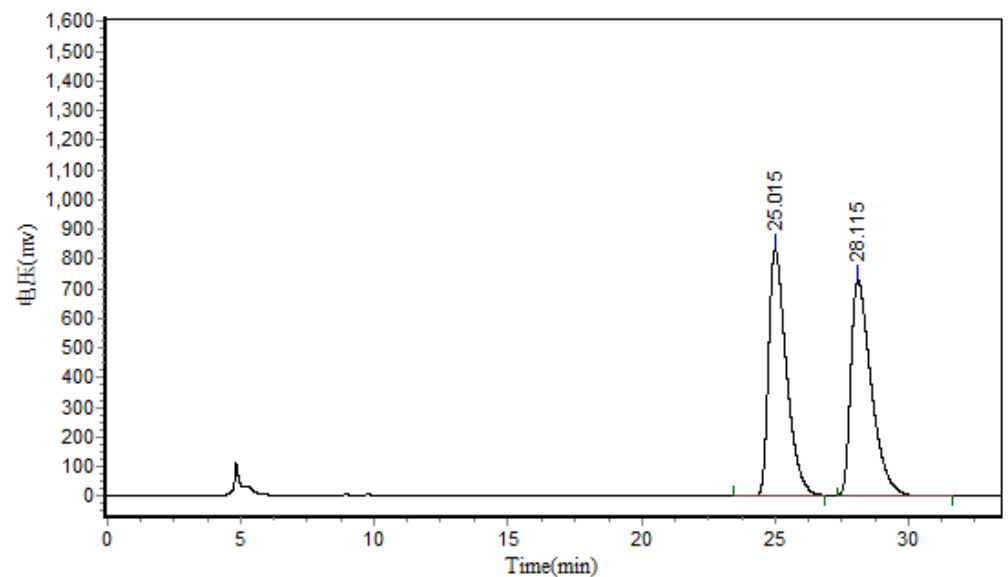

\begin{tabular}{|c|c|c|c|c|c|}
\hline \multicolumn{6}{|c|}{ Results } \\
\hline Peak No. & Peak ID & Ret Time & Height & Area & Conc. \\
\hline 1 & & 25.015 & 831891.625 & 38836504.000 & 49.8222 \\
\hline 2 & & 28.115 & 728628.938 & 39113720.000 & 50.1778 \\
\hline Total & & & 1560520.563 & 77950224.000 & 100.0000 \\
\hline
\end{tabular}


(R)-4-(4-chlorophenyl)-2-methyl-4-phenyl-1,2,5-thiadiazolidin-3-one 1,1-dioxide (3b)

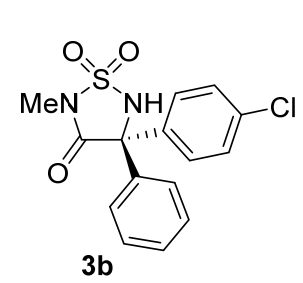

White solid, $95 \%$ yield, $96 \%$ ee; $\mathrm{mp} 135-137^{\circ} \mathrm{C}$.

$[\alpha]_{\mathrm{D}}{ }^{25}=-48.1\left(c 0.51, \mathrm{CHCl}_{3}\right) ;{ }^{1} \mathrm{H}$ NMR $\left(300 \mathrm{MHz}, \mathrm{CDCl}_{3}\right) \delta 7.47(\mathrm{~d}, J=$ $2.0 \mathrm{~Hz}, 1 \mathrm{H}), 7.45(\mathrm{~d}, J=2.0 \mathrm{~Hz}, 1 \mathrm{H}), 7.41-7.28(\mathrm{~m}, 7 \mathrm{H}), 5.40(\mathrm{~s}, 1 \mathrm{H}), 3.15$ (s, 3H); ${ }^{13} \mathrm{C}$ NMR $\left(125 \mathrm{MHz}, \mathrm{CDCl}_{3}\right) \delta 168.4,138.3,136.2,135.5,129.6$, 129.23, 129.17, 129.1, 127.6, 75.0, 26.4; IR (KBr) v 3433, 3184, 1710, 1491, $1448,1419,1342,1218,1179,1092,1067,1014,969 \mathrm{~cm}^{-1}$; HRMS (ESI) for $\mathrm{C}_{15} \mathrm{H}_{12} \mathrm{O}_{3} \mathrm{~N}_{2} \mathrm{SCl}[\mathrm{M}-\mathrm{H}]^{-}$calcd 335.0263, found 335.0267.

HPLC: Chiralpak AY-H column $(250 \mathrm{~mm})$; detected at $220 \mathrm{~nm}$; hexane $/ i$-propanol $=80 / 20$; flow $=$ $0.7 \mathrm{~mL} / \mathrm{min}$; Retention time: $41.9 \mathrm{~min}, 62.6 \mathrm{~min}$ (major).

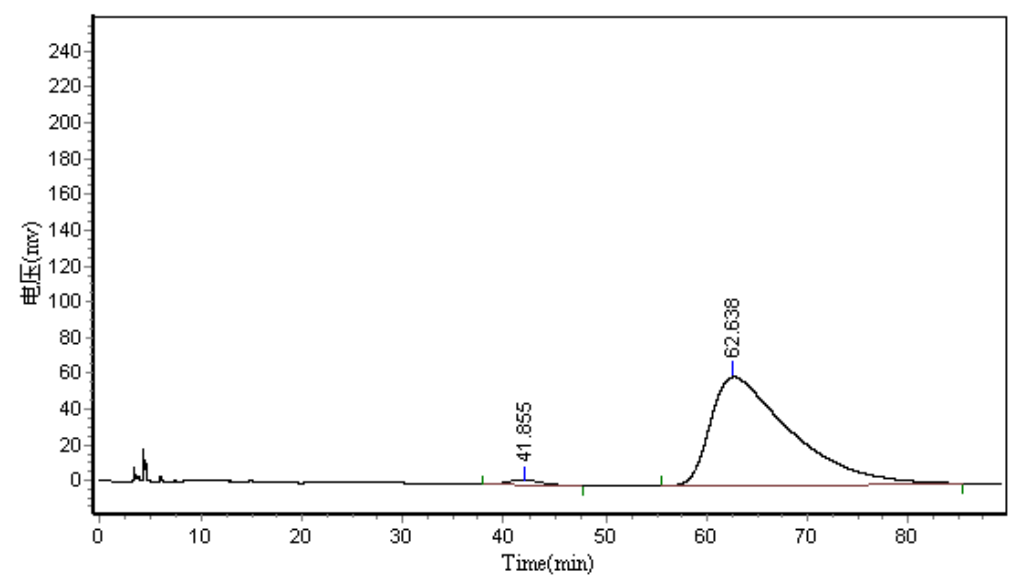

Results

\begin{tabular}{|c|c|c|c|c|c|}
\hline Peak No. & Peak ID & Ret Time & Height & Area & Conc. \\
\hline 1 & & 41.855 & 2890.220 & 690986.000 & 2.0318 \\
\hline 2 & & 62.638 & 60527.301 & 33318006.000 & 97.9682 \\
\hline Total & & & 63417.521 & 34008992.000 & 100.0000 \\
\hline
\end{tabular}

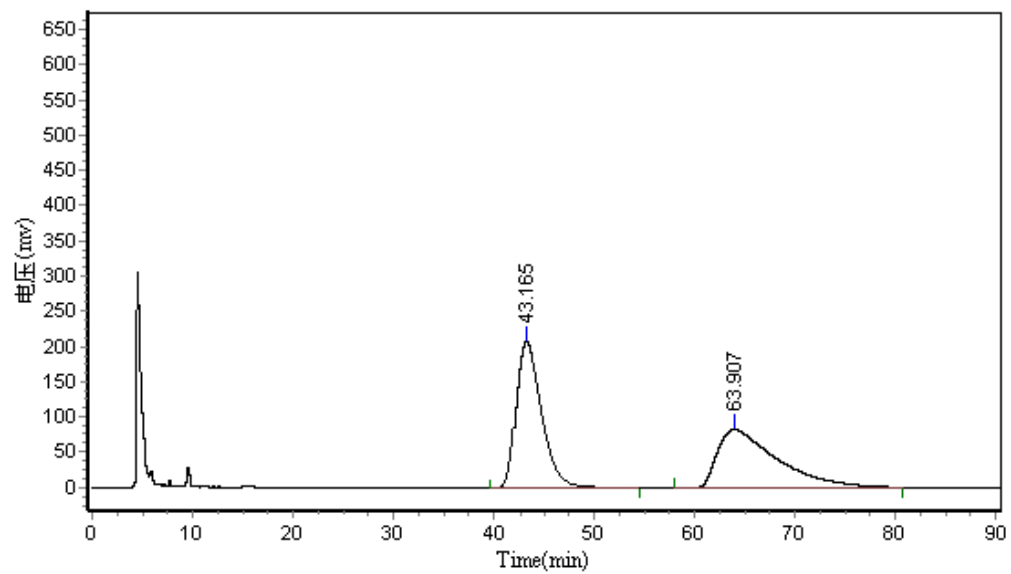

\begin{tabular}{|c|c|c|c|c|c|}
\hline \multicolumn{6}{|c|}{ Results } \\
\hline Peak No. & Peak ID & Ret Time & Height & Area & Conc. \\
\hline 1 & & 43.165 & 208089.063 & 36603732.000 & 51.3001 \\
\hline 2 & & 63.907 & 83746.961 & 34748368.000 & 48.6999 \\
\hline Total & & & 291836.023 & 71352100.000 & 100.0000 \\
\hline
\end{tabular}


(R)-4-(4-methoxyphenyl)-2-methyl-4-phenyl-1,2,5-thiadiazolidin-3-one 1,1-dioxide (3c)

White solid, $99 \%$ yield, $92 \%$ ee; $\mathrm{mp} 113-115{ }^{\circ} \mathrm{C}$.

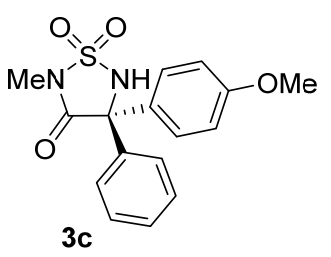

$[\alpha]_{\mathrm{D}}{ }^{25}=+9.1\left(c 0.535, \mathrm{CHCl}_{3}\right) ;{ }^{1} \mathrm{H} \mathrm{NMR}\left(300 \mathrm{MHz}, \mathrm{CDCl}_{3}\right) \delta 7.46(\mathrm{dd}$, $J=6.3,3.3 \mathrm{~Hz}, 2 \mathrm{H}), 7.36(\mathrm{dd}, J=6.1,2.8 \mathrm{~Hz}, 4 \mathrm{H}), 7.31(\mathrm{~s}, 1 \mathrm{H}), 6.86(\mathrm{~d}$, $J=8.9 \mathrm{~Hz}, 2 \mathrm{H}), 5.30(\mathrm{~s}, 1 \mathrm{H}), 3.78(\mathrm{~s}, 3 \mathrm{H}), 3.15(\mathrm{~s}, 3 \mathrm{H}) ;{ }^{13} \mathrm{C}$ NMR $(125$ $\left.\mathrm{MHz}, \mathrm{CDCl}_{3}\right) \delta 169.0,160.2,138.3,130.3,129.2,129.1,128.9,127.7$, 114.3, 75.3, 55.5, 26.3; IR (KBr) v 3166, 1714, 1615, 1513, 1418, 1336, 1254, 1171, 1032, $974 \mathrm{~cm}^{-1}$; HRMS (ESI) for $\mathrm{C}_{16} \mathrm{H}_{15} \mathrm{O}_{4} \mathrm{~N}_{2} \mathrm{~S}[\mathrm{M}-\mathrm{H}]^{-}$calcd 331.0758, found 331.0762 .

HPLC ( $N$-Me derivative): Chiralpak IC column $(250 \mathrm{~mm})$; detected at $220 \mathrm{~nm}$; hexane $/ i$-propanol $=97 / 3$; flow $=0.7 \mathrm{~mL} / \mathrm{min}$; Retention time: $35.3 \mathrm{~min}$ (major), $45.1 \mathrm{~min}$.

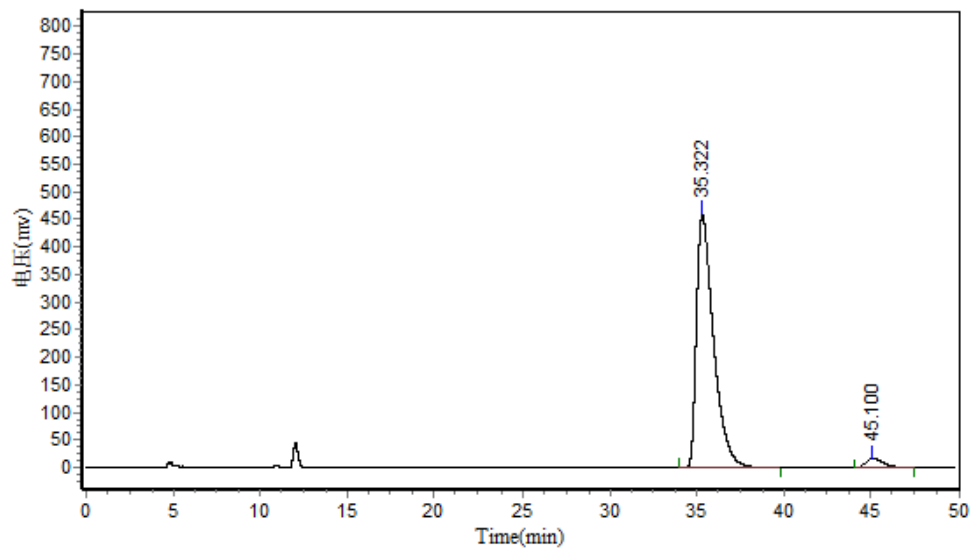

Results

\begin{tabular}{|c|c|c|c|c|c|}
\hline \multicolumn{6}{|c|}{ Nesuits } \\
\hline Peak No. & Peak ID & Ret Time & Height & Area & Conc. \\
\hline 1 & & 35.322 & 458665.063 & 31441812.000 & 96.0839 \\
\hline 2 & & 45.100 & 16979.051 & 1281469.875 & 3.9161 \\
\hline Total & & & 475644.113 & 32723281.875 & 100.0000 \\
\hline
\end{tabular}

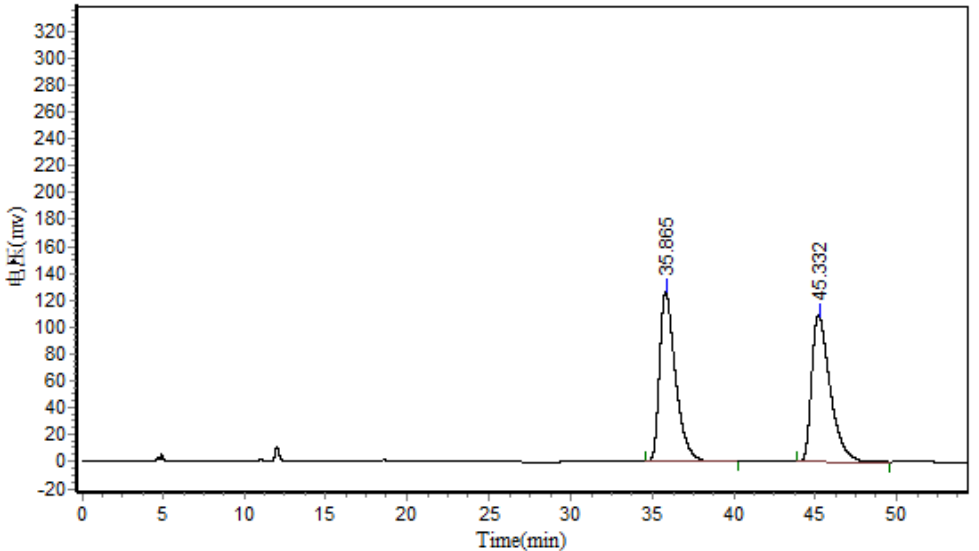

\begin{tabular}{|c|c|c|c|c|c|}
\hline \multicolumn{6}{|c|}{ Results } \\
\hline Peak No. & Peak ID & Ret Time & Height & Area & Conc. \\
\hline 1 & & 35.865 & 125986.961 & 8719130.000 & 49.9480 \\
\hline 2 & & 45.332 & 108997.930 & 8737295.000 & 50.0520 \\
\hline Total & & & 234984.891 & 17456425.000 & 100.0000 \\
\hline
\end{tabular}


(R)-4-(4-fluorophenyl)-2-methyl-4-phenyl-1,2,5-thiadiazolidin-3-one 1,1-dioxide (3d)

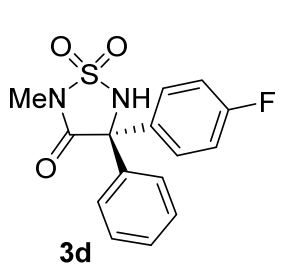

White solid, $94 \%$ yield, $94 \%$ ee; $\mathrm{mp} 143-146^{\circ} \mathrm{C}$.

$[\alpha]_{\mathrm{D}}{ }^{25}=-35.0\left(c 0.555, \mathrm{CHCl}_{3}\right) ;{ }^{1} \mathrm{H}$ NMR $\left(300 \mathrm{MHz}, \mathrm{CDCl}_{3}\right) \delta 7.54-7.46$ (m, 2H), 7.39 (s, 5H), $7.13-7.00(\mathrm{~m}, 2 \mathrm{H}), 5.46(\mathrm{~s}, 1 \mathrm{H}), 3.17(\mathrm{~s}, 3 \mathrm{H}) ;{ }^{13} \mathrm{C}$ $\operatorname{NMR}\left(125 \mathrm{MHz}, \mathrm{CDCl}_{3}\right) \delta 168.6,163.1\left(\mathrm{~d},{ }^{1} J_{\mathrm{CF}}=248.8 \mathrm{~Hz}\right), 138.4,133.7$, $129.8\left(\mathrm{~d},{ }^{3} J_{\mathrm{CF}}=8.4 \mathrm{~Hz}\right), 129.5,129.1,127.6,115.8\left(\mathrm{~d},{ }^{2} J_{\mathrm{CF}}=21.3 \mathrm{~Hz}\right), 75.0$, 26.3; ${ }^{19} \mathrm{~F} \mathrm{NMR}\left(471 \mathrm{MHz}, \mathrm{CDCl}_{3}\right) \delta-112.1$ (s); IR (KBr) v 3433, 3180, 1712, $1606,1508,1409,1342,1225,1187,1164,1069,969 \mathrm{~cm}^{-1}$; HRMS (ESI) for $\mathrm{C}_{15} \mathrm{H}_{12} \mathrm{O}_{3} \mathrm{~N}_{2} \mathrm{FS}$ [M-H] calcd 319.0558, found 319.0560.

HPLC ( $N$-Boc derivative): Chiralpak IC column $(250 \mathrm{~mm})$; detected at $220 \mathrm{~nm}$; hexane $/ i$-propanol $=97 / 3$; flow $=0.7 \mathrm{~mL} / \mathrm{min}$; Retention time: $14.8 \mathrm{~min}, 18.3 \mathrm{~min}$ (major).

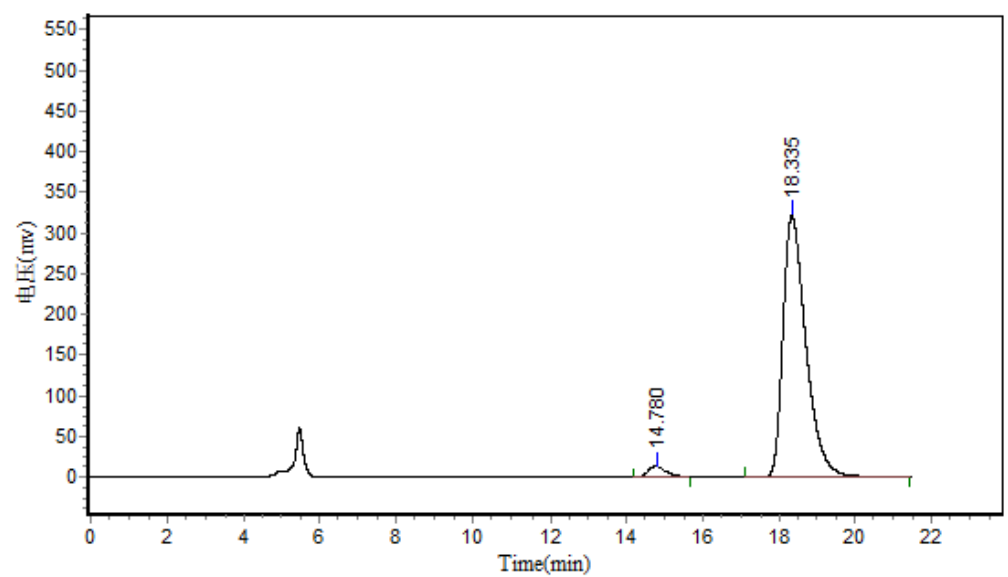

\begin{tabular}{|c|c|c|c|c|c|}
\hline \multicolumn{6}{|c|}{ Results } \\
\hline Peak No. & Peak ID & Ret Time & Height & Area & Conc. \\
\hline 1 & & 14.780 & 13800.821 & 440820.281 & 3.1764 \\
\hline 2 & & 18.335 & 321122.844 & 13437330.000 & 96.8236 \\
\hline Total & & & 334923.665 & 13878150.281 & 100.0000 \\
\hline
\end{tabular}

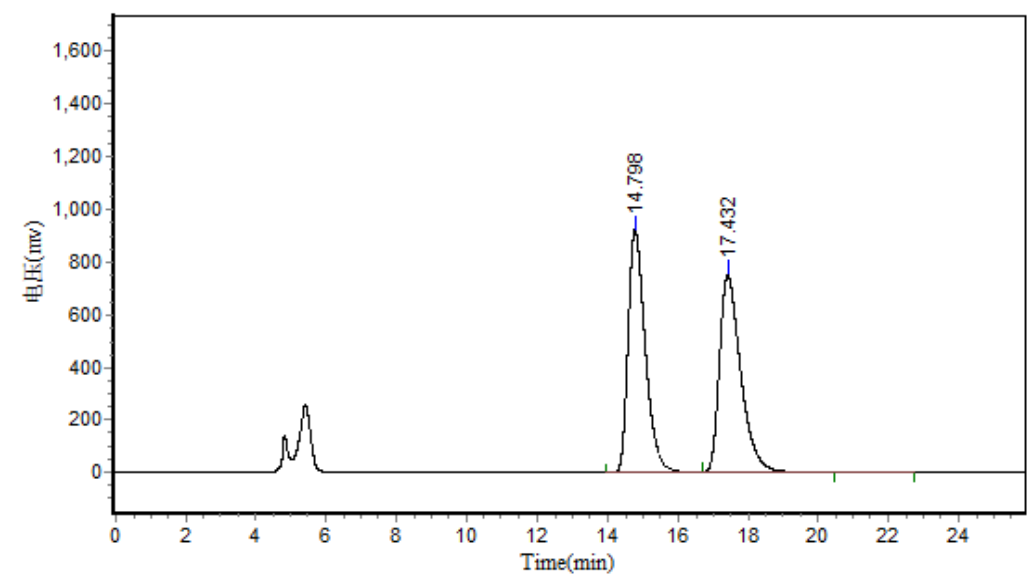

Results

\begin{tabular}{cccccc} 
Peak No. & Peak ID & Ret Time & Height & Area & Conc. \\
\hline 1 & 14.798 & 918173.375 & 31803374.000 & 50.3561 \\
2 & 17.432 & 744133.063 & 31353524.000 & 49.6439 \\
\hline Total & & 1662306.438 & 63156898.000 & 100.0000
\end{tabular}


(R)-4-(4-bromophenyl)-2-methyl-4-phenyl-1,2,5-thiadiazolidin-3-one 1,1-dioxide (3e)

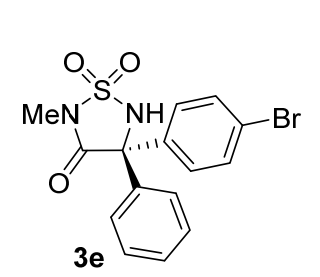

White solid, $96 \%$ yield, $96 \%$ ee; $\mathrm{mp} 149-152^{\circ} \mathrm{C}$.

$[\alpha]_{\mathrm{D}}{ }^{25}=-43.0\left(c 0.54, \mathrm{CHCl}_{3}\right) ;{ }^{1} \mathrm{H}$ NMR $\left(300 \mathrm{MHz}, \mathrm{CDCl}_{3}\right) \delta 7.52(\mathrm{~d}, J=$

$2.0 \mathrm{~Hz}, 1 \mathrm{H}), 7.49(\mathrm{~d}, J=2.1 \mathrm{~Hz}, 1 \mathrm{H}), 7.42(\mathrm{~d}, J=2.0 \mathrm{~Hz}, 1 \mathrm{H}), 7.40-7.29$ $(\mathrm{m}, 6 \mathrm{H}), 5.34(\mathrm{~s}, 1 \mathrm{H}), 3.16(\mathrm{~s}, 3 \mathrm{H}) ;{ }^{13} \mathrm{C}$ NMR $\left(125 \mathrm{MHz}, \mathrm{CDCl}_{3}\right) \delta 168.3$, 138.3, 136.7, 132.0, 129.7, 129.5, 129.3, 127.6, 123.8, 75.1, 26.4; IR (KBr) $v$ 3450, 3156, 1713, 1486, 1421, 1342, $1174 \mathrm{~cm}^{-1}$; HRMS (ESI) for $\mathrm{C}_{15} \mathrm{H}_{12} \mathrm{O}_{3} \mathrm{~N}_{2} \mathrm{BrS}$ [M-H] $]^{-}$calcd 378.9757, found 378.9766.

HPLC: Chiralpak IC column $(250 \mathrm{~mm})$; detected at $220 \mathrm{~nm}$; hexane $/ i$-propanol $=99 / 1$; flow $=1.2$ $\mathrm{mL} / \mathrm{min}$; Retention time: $30.5 \mathrm{~min}, 38.0 \mathrm{~min}$ (major).

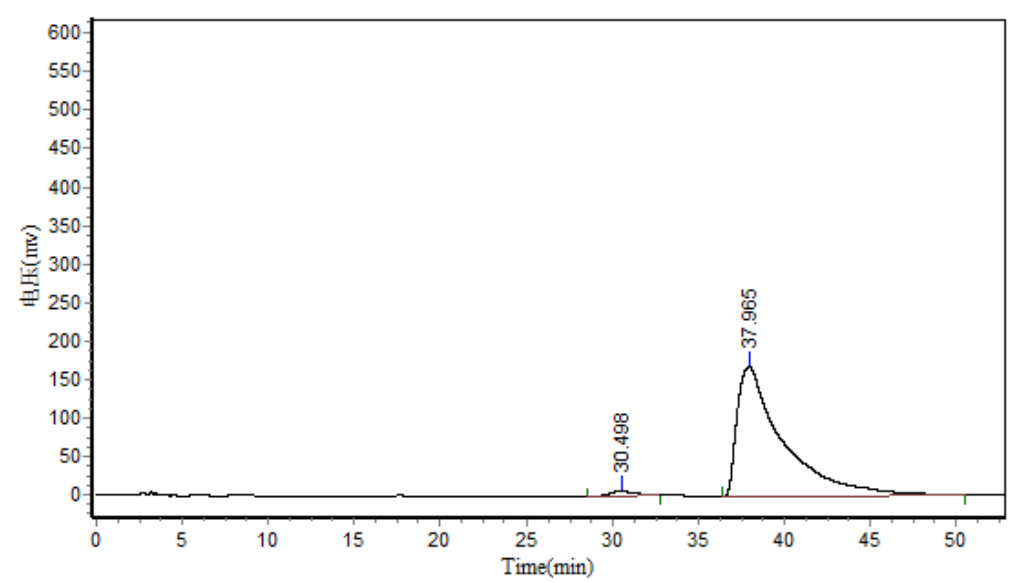

\begin{tabular}{cccccc} 
& \multicolumn{2}{c}{ Results } & Area & Conc. \\
\hline Peak No. & Peak ID & Ret Time & Height & 616223.313 & 1.9323 \\
2 & 30.498 & 5904.020 & 31275020.000 & 98.0677 \\
\hline Total & 37.965 & 167973.219 & 31891243.313 & 100.0000
\end{tabular}

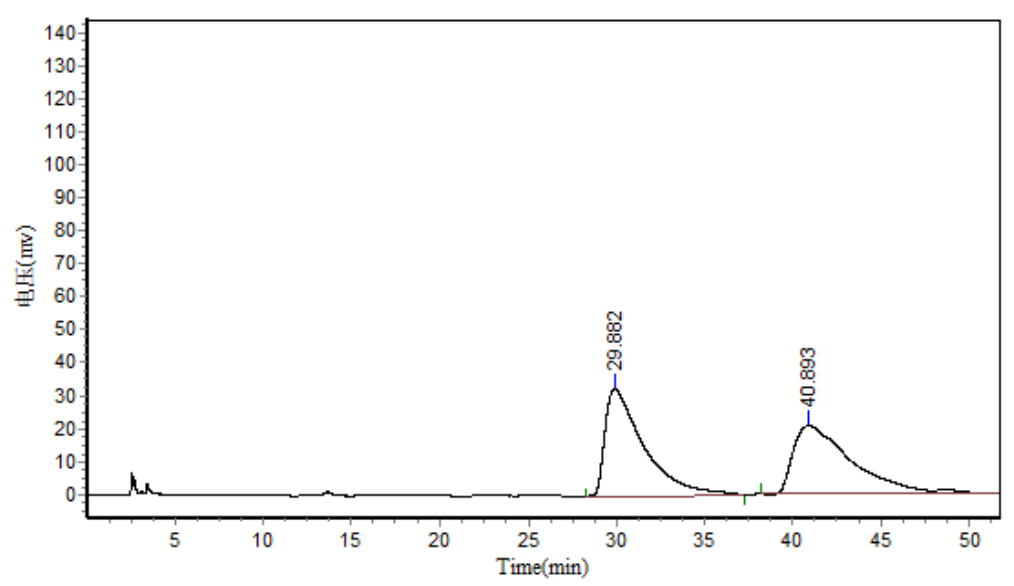

Results

\begin{tabular}{|c|c|c|c|c|c|}
\hline \multicolumn{6}{|c|}{ 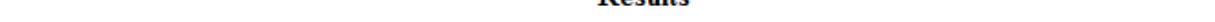 } \\
\hline Peak No. & Peak ID & Ret Time & Height & Area & Conc. \\
\hline 1 & & 29.882 & 32275.133 & 4918198.500 & 50.5123 \\
\hline 2 & & 40.893 & 20542.166 & 4818433.500 & 49.4877 \\
\hline Total & & & 52817.299 & 9736632.000 & 100.0000 \\
\hline
\end{tabular}


(R)-4-([1,1'-biphenyl]-4-yl)-2-methyl-4-phenyl-1,2,5-thiadiazolidin-3-one 1,1-dioxide (3f)<smiles></smiles>

White solid, $92 \%$ yield, $94 \%$ ee; $\mathrm{mp} 147-149^{\circ} \mathrm{C}$.

$[\alpha]_{\mathrm{D}}{ }^{25}=-6.6\left(c 0.505, \mathrm{CHCl}_{3}\right) ;{ }^{1} \mathrm{H} \mathrm{NMR}\left(300 \mathrm{MHz}, \mathrm{CDCl}_{3}\right) \delta 7.54(\mathrm{dd}, J=$ 12.0, $5.7 \mathrm{~Hz}, 5 \mathrm{H}), 7.50-7.40(\mathrm{~m}, 4 \mathrm{H}), 7.40-7.24(\mathrm{M}, 5 \mathrm{H}), 5.42(\mathrm{~s}, 1 \mathrm{H})$,

$3.14(\mathrm{~s}, 3 \mathrm{H}) ;{ }^{13} \mathrm{C}$ NMR $\left(100 \mathrm{MHz}, \mathrm{CDCl}_{3}\right) \delta 168.7,142.1,140.0,138.2$, 137.0, 129.4, 129.0, 128.97, 128.1, 127.9, 127.7, 127.6, 127.2, 75.4, 26.3; IR $(\mathrm{KBr}) v 3214,1717,1712,1332,1171,1071,978 \mathrm{~cm}^{-1}$; HRMS (ESI) for $\mathrm{C}_{21} \mathrm{H}_{17} \mathrm{O}_{3} \mathrm{~N}_{2} \mathrm{~S}[\mathrm{M}-\mathrm{H}]^{-}$calcd 377.0965, found 377.0973.

HPLC (N-Boc derivative): Chiralcel OZ-H column $(250 \mathrm{~mm})$; detected at $220 \mathrm{~nm}$; hexane $/ i$-propanol $=99 / 1$; flow $=0.5 \mathrm{~mL} / \mathrm{min}$; Retention time: $34.1 \mathrm{~min}, 39.4 \mathrm{~min}$ (major).

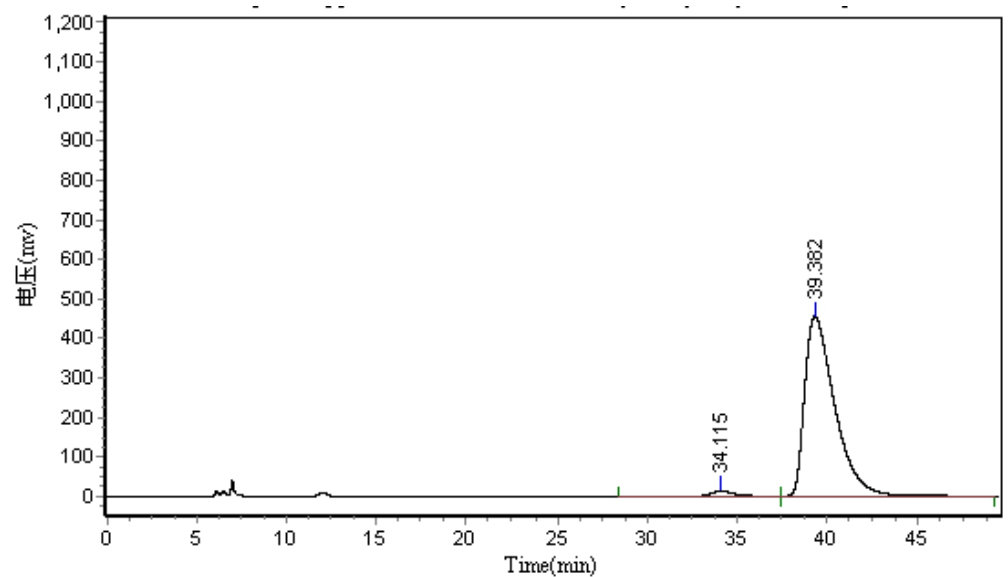

\begin{tabular}{|c|c|c|c|c|c|}
\hline \multicolumn{6}{|c|}{ Results } \\
\hline Peak No. & Peak ID & Ret Time & Height & Area & Conc. \\
\hline 1 & & 34.115 & 13876.675 & 1554674.250 & 2.8295 \\
\hline 2 & & 39.382 & 455826.844 & 53390516.000 & 97.1705 \\
\hline Total & & & 469703.519 & 54945190.250 & 100.0000 \\
\hline
\end{tabular}

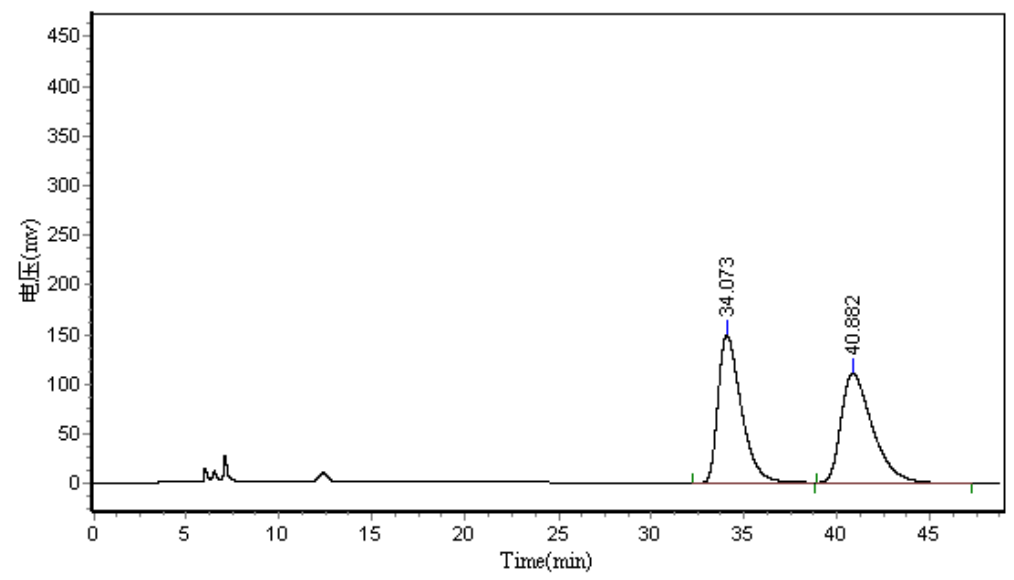

Results

\begin{tabular}{cccccc} 
Peak No. & Peak D & Ret Time & Height & Area & Conc. \\
\hline 1 & 34.073 & 148510.313 & 12894390.000 & 49.7393 \\
2 & 40.882 & 109786.914 & 13029547.000 & 50.2607 \\
\hline Total & & 258297.227 & 25923937.000 & 100.0000
\end{tabular}


(R)-4-(3-fluorophenyl)-2-methyl-4-phenyl-1,2,5-thiadiazolidin-3-one 1,1-dioxide (3g)<smiles>CC(=O)NC(C(C)=O)(c1ccccc1)c1ccccc1</smiles>

White solid, $91 \%$ yield, $95 \%$ ee; $\mathrm{mp} 135-137^{\circ} \mathrm{C}$.

$[\alpha]_{\mathrm{D}}{ }^{25}=-39.9\left(c 0.495, \mathrm{CHCl}_{3}\right) ;{ }^{1} \mathrm{H}$ NMR $\left(300 \mathrm{MHz}, \mathrm{CDCl}_{3}\right) \delta 7.45-7.30(\mathrm{~m}$, $7 \mathrm{H}), 7.29-7.20(\mathrm{~m}, 1 \mathrm{H}), 7.12-7.00(\mathrm{~m}, 1 \mathrm{H}), 5.39(\mathrm{~s}, 1 \mathrm{H}), 3.17(\mathrm{~s}, 3 \mathrm{H}) ;{ }^{13} \mathrm{C}$ $\operatorname{NMR}\left(125 \mathrm{MHz}, \mathrm{CDCl}_{3}\right) \delta 168.2,162.8\left(\mathrm{~d},{ }^{1} J_{\mathrm{CF}}=246.3 \mathrm{~Hz}\right), 140.1\left(\mathrm{~d},{ }^{3} J_{\mathrm{CF}}=\right.$ $7.2 \mathrm{~Hz}), 138.2,130.5\left(\mathrm{~d},{ }^{3} J_{\mathrm{CF}}=8.2 \mathrm{~Hz}\right), 129.6,129.2,127.6,123.4\left(\mathrm{~d},{ }^{4} J_{\mathrm{CF}}=2.9\right.$ $\mathrm{Hz}), 116.4\left(\mathrm{~d},{ }^{2} J_{\mathrm{CF}}=20.0 \mathrm{~Hz}\right), 115.2\left(\mathrm{~d},{ }^{2} J_{\mathrm{CF}}=23.8 \mathrm{~Hz}\right), 75.0,26.4 ;{ }^{19} \mathrm{~F}$ NMR $\left(471 \mathrm{MHz}, \mathrm{CDCl}_{3}\right) \delta-111.0(\mathrm{~s})$; IR (KBr) $v 3448,3172,1703,1591,1408,1338,1220,1185,1055$ $\mathrm{cm}^{-1}$; HRMS (ESI) for $\mathrm{C}_{15} \mathrm{H}_{12} \mathrm{O}_{3} \mathrm{~N}_{2} \mathrm{FS}[\mathrm{M}-\mathrm{H}]^{-}$calcd 319.0558, found 319.0562 .

HPLC (N-Boc derivative): Chiralcel OZ-H column $(250 \mathrm{~mm})$; detected at $220 \mathrm{~nm}$; hexane $/ i$-propanol $=99 / 1$; flow $=0.3 \mathrm{~mL} / \mathrm{min}$; Retention time: $39.0 \mathrm{~min}, 40.8 \mathrm{~min}$ (major).

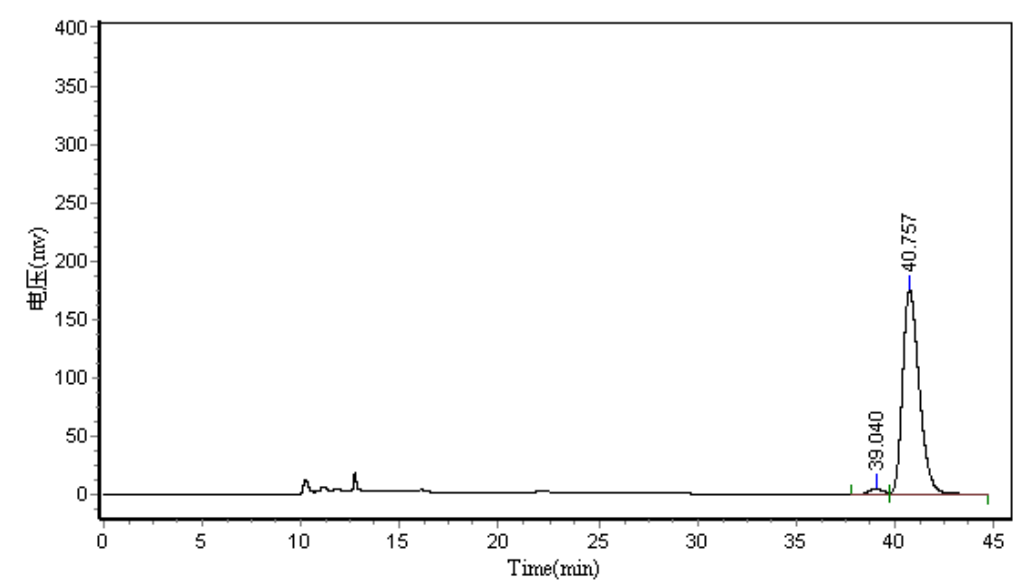

Results

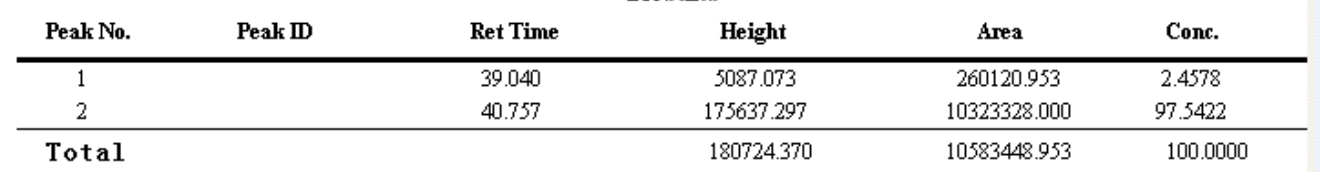

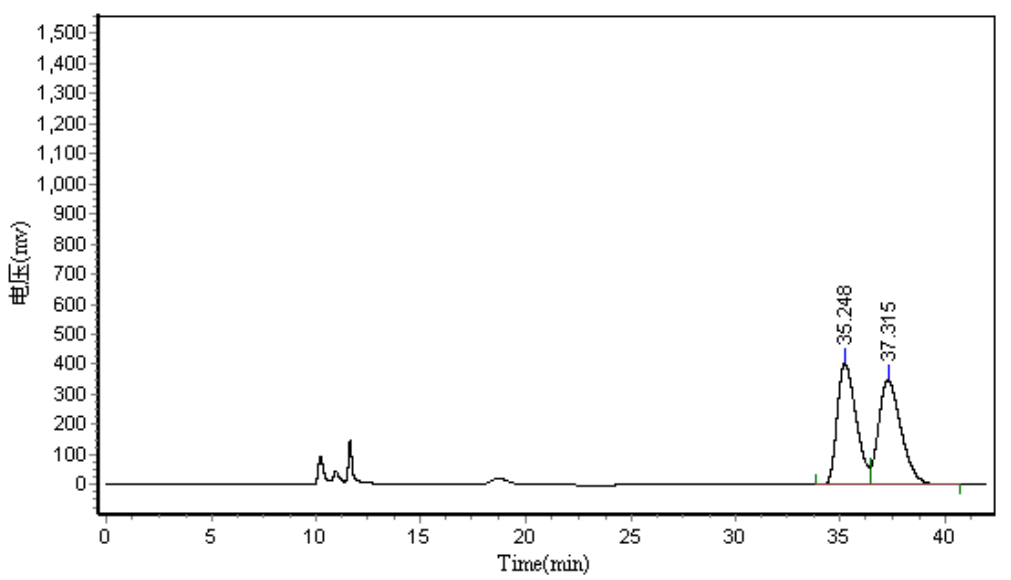

Results

\begin{tabular}{|c|c|c|c|c|c|}
\hline \\
\hline Peak No. & Peak ID & Ret Time & Height & Area & Conc. \\
\hline 1 & & 35.248 & 401680.344 & 25394610.000 & 49.2109 \\
\hline 2 & & 37.315 & 348938.875 & 26209016.000 & 50.7891 \\
\hline Total & & & 750619.219 & 51603626.000 & 100.0000 \\
\hline
\end{tabular}


(R)-2-methyl-4-phenyl-4-(m-tolyl)-1,2,5-thiadiazolidin-3-one 1,1-dioxide (3h)<smiles>Cc1ccc(C2(c3ccccc3)OS(=O)(=O)N(C)C2=O)cc1</smiles>

White solid, $96 \%$ yield, $94 \%$ ee; $\mathrm{mp} 121-123^{\circ} \mathrm{C}$.

$[\alpha]_{\mathrm{D}}{ }^{25}=+17.1\left(c 0.5, \mathrm{CHCl}_{3}\right) ;{ }^{1} \mathrm{H}$ NMR $\left(300 \mathrm{MHz}, \mathrm{CDCl}_{3}\right) \delta 7.54-7.43(\mathrm{~m}$, $2 \mathrm{H}), 7.40-7.33(\mathrm{~m}, 3 \mathrm{H}), 7.27-7.13(\mathrm{~m}, 4 \mathrm{H}), 5.17(\mathrm{~s}, 1 \mathrm{H}), 3.17(\mathrm{~s}, 3 \mathrm{H}), 2.33(\mathrm{~s}$, $3 \mathrm{H}) ;{ }^{13} \mathrm{C}$ NMR $\left(100 \mathrm{MHz}, \mathrm{CDCl}_{3}\right) \delta 168.7,138.9,138.3,138.1,130.2,129.3$, 128.9, 128.1, 127.7, 124.8, 75.6, 26.3, 21.6; IR (KBr) v 3171, 1715, 1405, 1340, 1184, 1068, $1050 \mathrm{~cm}^{-1}$; HRMS (ESI) for $\mathrm{C}_{16} \mathrm{H}_{15} \mathrm{O}_{3} \mathrm{~N}_{2} \mathrm{~S}$ [M-H] calcd 315.0809, found 315.0817 .

HPLC (N-Me derivative): Chiralpak AY-H column $(250 \mathrm{~mm})$; detected at $220 \mathrm{~nm}$; hexane $/ i$-propanol $=70 / 30$; flow $=0.7 \mathrm{~mL} / \mathrm{min}$; Retention time: $16.8 \mathrm{~min}$ (major), $38.3 \mathrm{~min}$.

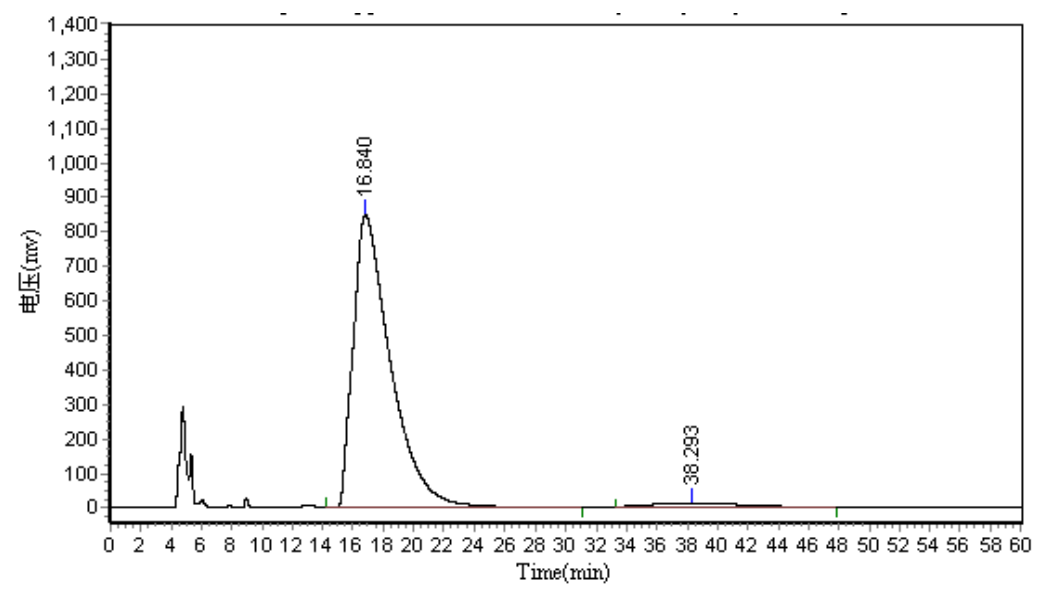

Results

\begin{tabular}{|c|c|c|c|c|c|}
\hline Peak No. & Peak DD & Ret Time & Height & Area & Conc. \\
\hline 1 & & 16.840 & 847875.188 & 145312272.000 & 96.7265 \\
\hline 2 & & 38.293 & 11180.368 & 4917712.500 & 3.2735 \\
\hline Total & & & 859055.556 & 150229984.500 & 100.0000 \\
\hline
\end{tabular}

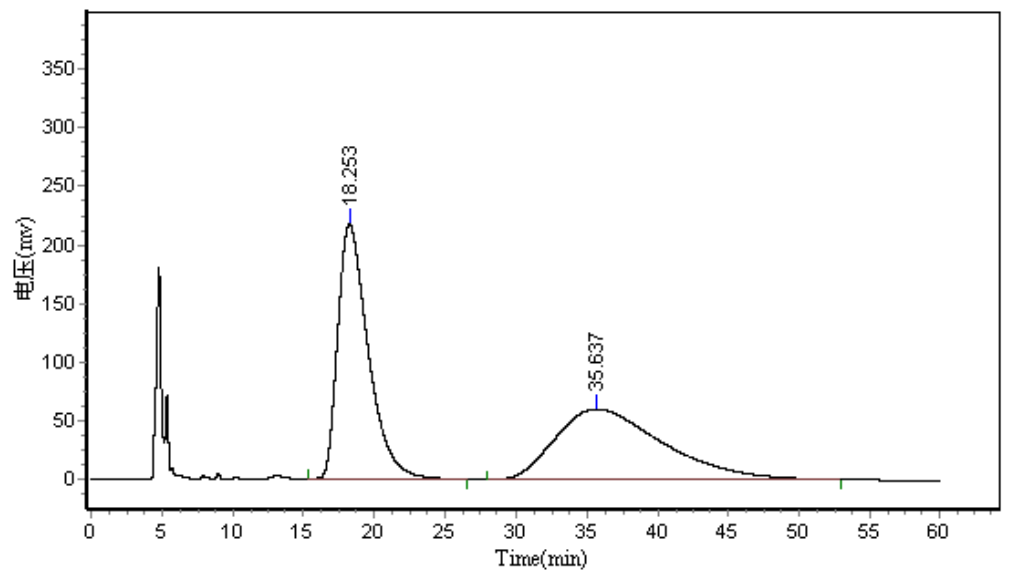

\begin{tabular}{|c|c|c|c|c|c|}
\hline \multicolumn{6}{|c|}{ Results } \\
\hline Peak No. & Peak ID & Ret Time & Height & Area & Conc. \\
\hline 1 & & 18.253 & 216798.719 & 32576768.000 & 50.5689 \\
\hline 2 & & 35.637 & 60253.402 & 31843790.000 & 49.4311 \\
\hline Total & & & 277052.121 & 64420558.000 & 100.000 \\
\hline
\end{tabular}


(R)-2-methyl-4-(naphthalen-2-yl)-4-phenyl-1,2,5-thiadiazolidin-3-one 1,1-dioxide (3i)

White solid, $98 \%$ yield, $94 \%$ ee; $\mathrm{mp} 128-130{ }^{\circ} \mathrm{C}$.

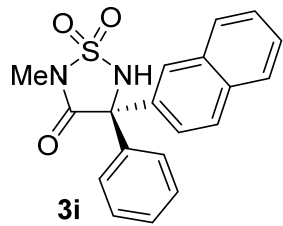

$[\alpha]_{\mathrm{D}}^{25}=+13.3\left(c 0.49, \mathrm{CHCl}_{3}\right) ;{ }^{1} \mathrm{H}$ NMR $\left(300 \mathrm{MHz}, \mathrm{CDCl}_{3}\right) 8.03(\mathrm{~d}, J=$ $1.8 \mathrm{~Hz}, 1 \mathrm{H}), 7.85-7.74(\mathrm{~m}, 3 \mathrm{H}), 7.55-7.47(\mathrm{~m}, 2 \mathrm{H}), 7.47-7.39(\mathrm{~m}, 3 \mathrm{H})$, 7.35 (dt, $J=4.9,2.6 \mathrm{~Hz}, 3 \mathrm{H}), 5.44(\mathrm{~s}, 1 \mathrm{H}), 3.13(\mathrm{~s}, 3 \mathrm{H}) ;{ }^{13} \mathrm{C}$ NMR $(125$ $\left.\mathrm{MHz}, \mathrm{CDCl}_{3}\right) \delta 168.6,138.2,135.2,133.3,132.8,129.4,129.04,129.01$, $128.7,127.8,127.7,127.3,127.1,126.9,124.8,75.7,26.3$; IR (KBr) $v$ $3441,3245,1735,1387,1327,1177 \mathrm{~cm}^{-1}$; HRMS (ESI) for $\mathrm{C}_{19} \mathrm{H}_{15} \mathrm{O}_{3} \mathrm{~N}_{2} \mathrm{~S}[\mathrm{M}-\mathrm{H}]^{-}$calcd 351.0809, found 351.0814 .

HPLC ( $N$-Boc derivative): Chiralpak IC column $(250 \mathrm{~mm})$; detected at $220 \mathrm{~nm}$; hexane $/ i$-propanol $=97 / 3$; flow $=0.7 \mathrm{~mL} / \mathrm{min}$; Retention time: $31.4 \mathrm{~min}$ (major), $36.0 \mathrm{~min}$.

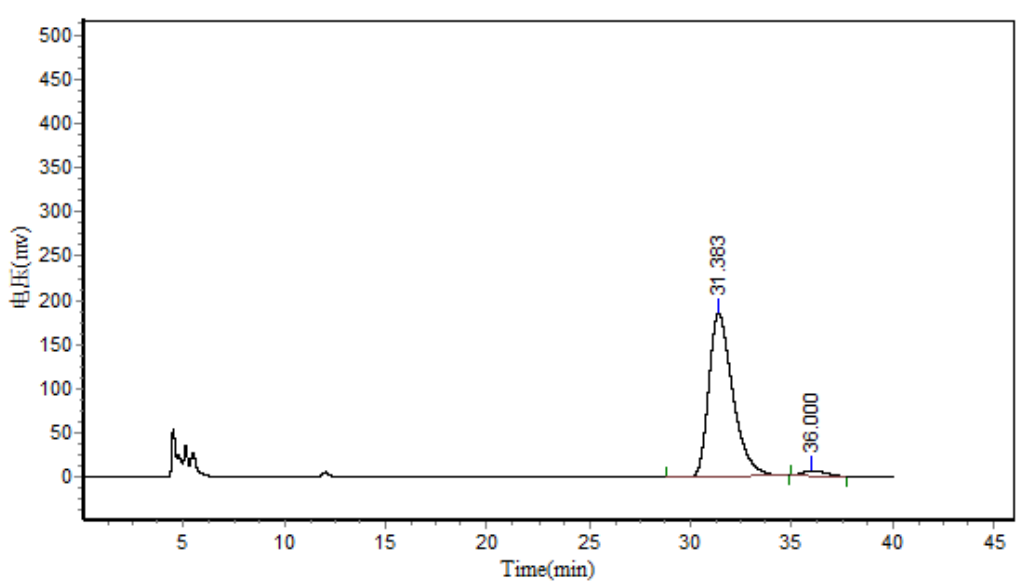

Results

\begin{tabular}{|c|c|c|c|c|c|}
\hline Peak No. & Peak ID & Ret Time & Height & Area & Conc. \\
\hline 1 & & 31.383 & 184808.297 & 15295094.000 & 96.9729 \\
\hline 2 & & 36.000 & 5945.305 & 477445.281 & 3.0271 \\
\hline Total & & & 190753.602 & 15772539.281 & 100.0000 \\
\hline
\end{tabular}

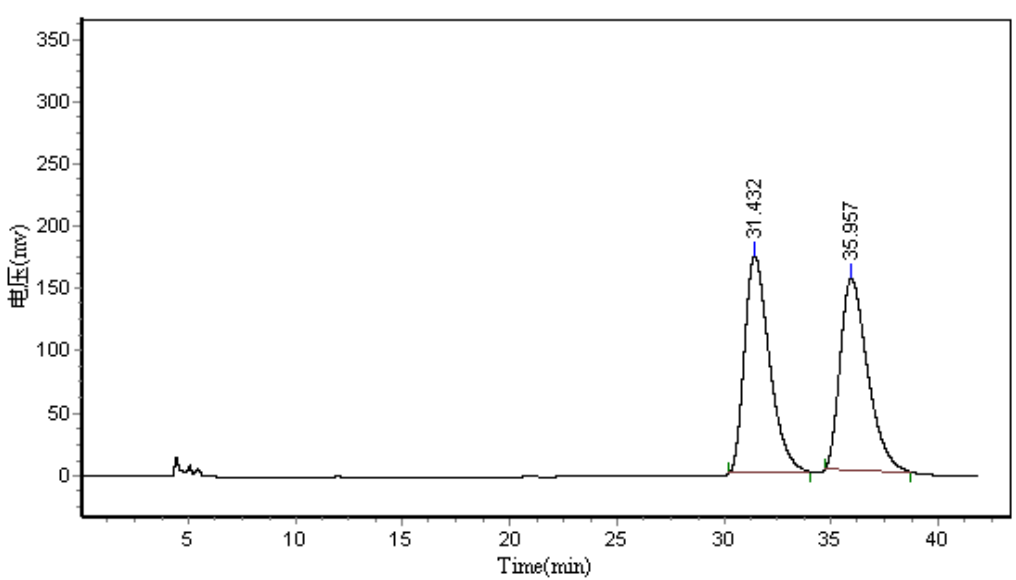

Results

\begin{tabular}{|c|c|c|c|c|c|}
\hline Peak No. & Peak ID & Ret Time & Height & Area & Conc. \\
\hline 1 & & 31.432 & 174121.813 & 14180786.000 & 50.7715 \\
\hline 2 & & 35.957 & 154242.453 & 13749800.000 & 49.2285 \\
\hline Total & & & 328364.266 & 27930586.000 & 100.0000 \\
\hline
\end{tabular}


(R)-2-methyl-4-(naphthalen-1-yl)-4-phenyl-1,2,5-thiadiazolidin-3-one 1,1-dioxide (3j)

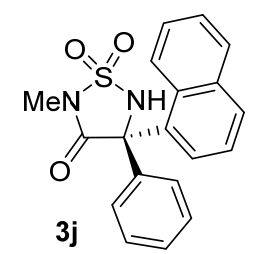

White solid, $93 \%$ yield, $84 \%$ ee; $\mathrm{mp} 125-128^{\circ} \mathrm{C}$.

$[\alpha]_{\mathrm{D}}{ }^{25}=+264.2\left(c 0.53, \mathrm{CHCl}_{3}\right) ;{ }^{1} \mathrm{H}$ NMR $\left(300 \mathrm{MHz}, \mathrm{CDCl}_{3}\right) \delta 7.90(\mathrm{~d}, J=8.0$ $\mathrm{Hz}, 1 \mathrm{H}), 7.85(\mathrm{~d}, J=8.0 \mathrm{~Hz}, 1 \mathrm{H}), 7.78(\mathrm{~d}, J=7.0 \mathrm{~Hz}, 1 \mathrm{H}), 7.66-7.46(\mathrm{~m}, 4 \mathrm{H})$, $7.44-7.21(\mathrm{~m}, 5 \mathrm{H}), 5.60(\mathrm{~s}, 1 \mathrm{H}), 3.13(\mathrm{~s}, 3 \mathrm{H}) ;{ }^{13} \mathrm{C} \mathrm{NMR}\left(125 \mathrm{MHz}, \mathrm{CDCl}_{3}\right) \delta$ $167.7,138.5,135.3,133.4,131.1,129.6,129.0,128.9,127.0,126.8,126.2$, 125.3, 125.0, 76.6 , 26.3; IR (KBr) v 3449, 3227, 1735, 1399, 1328, 1173, 1036, 1018, $954 \mathrm{~cm}^{-1}$; HRMS HRMS (ESI) for $\mathrm{C}_{19} \mathrm{H}_{15} \mathrm{O}_{3} \mathrm{~N}_{2} \mathrm{~S}$ [M-H] $]^{-}$calcd 351.0809, found 351.0811.

HPLC (N-Boc derivative): Chiralpak AD-H column $(250 \mathrm{~mm})$; detected at $220 \mathrm{~nm}$; hexane $/ i$-propanol $=99 / 1$; flow $=0.7 \mathrm{~mL} / \mathrm{min}$; Retention time: $33.1 \mathrm{~min}$ (major), $41.5 \mathrm{~min}$.

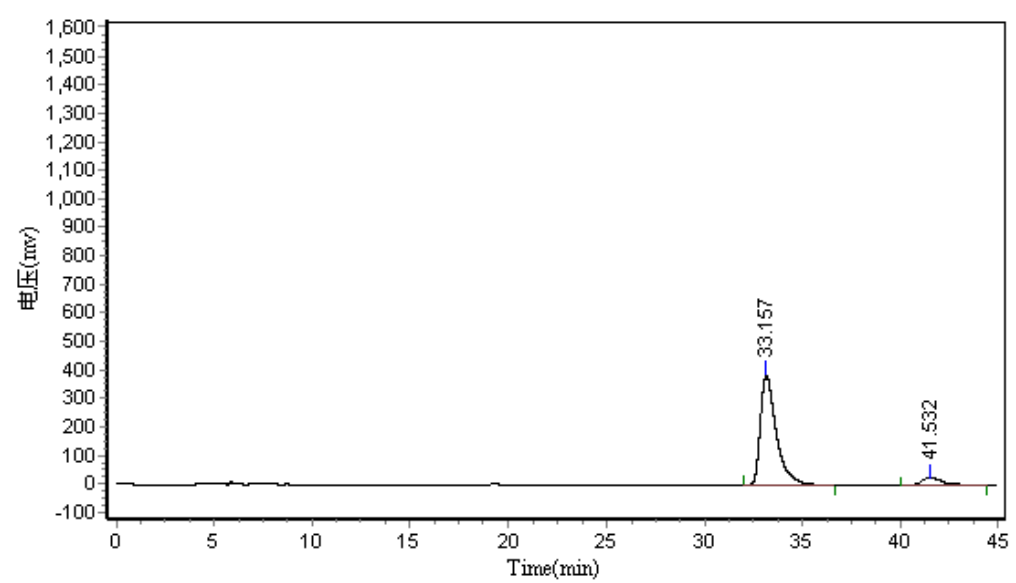

\begin{tabular}{|c|c|c|c|c|c|}
\hline \multicolumn{6}{|c|}{ Results } \\
\hline Peak No. & Peak ID & Ret Time & Height & Area & Conc. \\
\hline 1 & & 33.157 & 384109.344 & 21653472.000 & 92.0450 \\
\hline 2 & & 41.532 & 26999.109 & 1871416.000 & 7.9550 \\
\hline Total & & & 411108.453 & 23524888.000 & 100.0000 \\
\hline
\end{tabular}

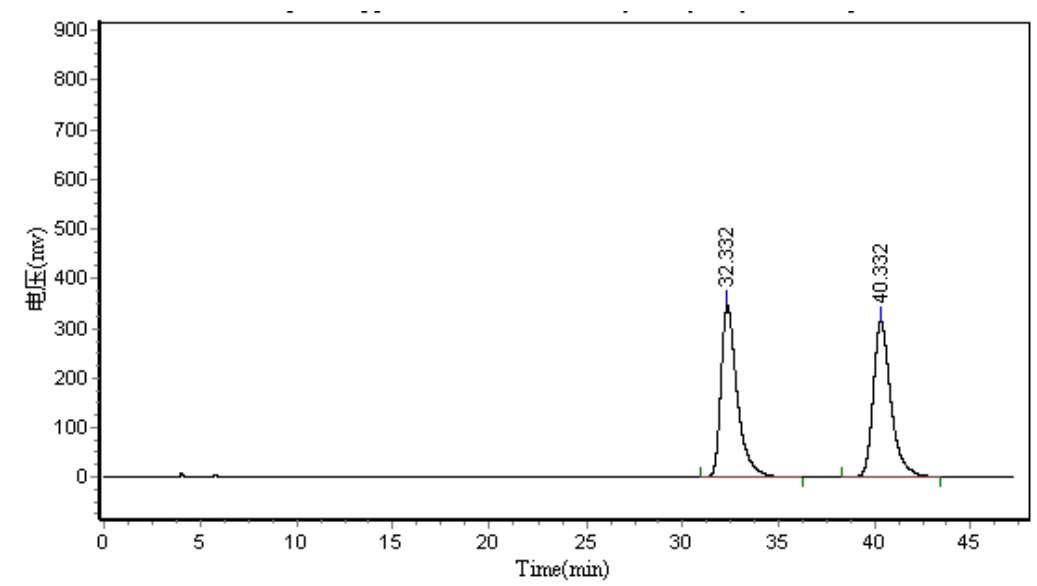

Results

\begin{tabular}{cccccc} 
Peak No. & Peak D & Ret Time & Height & Area & Conc. \\
\hline 1 & 32.332 & 343783.375 & 20195314.000 & 50.1020 \\
2 & 40.332 & 313774.469 & 20113050.000 & 49.8980 \\
\hline Total & & 657557.844 & 40308364.000 & 100.0000
\end{tabular}


(R)-2-methyl-4-phenyl-4-(o-tolyl)-1,2,5-thiadiazolidin-3-one 1,1-dioxide (3k)<smiles>CN1O[N+](C)([O-])C(c2ccccc2)(c2ccccc2)C1=O</smiles>

White solid, $90 \%$ yield, $85 \%$ ee; $\mathrm{mp} 110-113{ }^{\circ} \mathrm{C}$.

$[\alpha]_{\mathrm{D}}{ }^{25}=+165.3\left(c 0.48, \mathrm{CHCl}_{3}\right) ;{ }^{1} \mathrm{H} \mathrm{NMR}\left(300 \mathrm{MHz}, \mathrm{CDCl}_{3}\right) \delta 7.54(\mathrm{dd}, J=6.3$, $2.6 \mathrm{~Hz}, 2 \mathrm{H}), 7.42-7.30(\mathrm{~m}, 3 \mathrm{H}), 7.27(\mathrm{~d}, J=7.6 \mathrm{~Hz}, 2 \mathrm{H}), 7.17(\mathrm{t}, J=6.7 \mathrm{~Hz}$, $2 \mathrm{H}), 5.43(\mathrm{~s}, 1 \mathrm{H}), 3.10(\mathrm{~s}, 3 \mathrm{H}), 2.00(\mathrm{~s}, 3 \mathrm{H}) ;{ }^{13} \mathrm{C} \mathrm{NMR}\left(100 \mathrm{MHz}, \mathrm{CDCl}_{3}\right) \delta$ $168.3,137.6,137.1,136.8,133.3,129.8,128.82,128.78,128.7,127.3,126.4$, 76.2, 26.2, 21.0; IR (KBr) v 3425, 3245, 1745, 1450, 1395, 1332, 1296, 1174, $1062,969 \mathrm{~cm}^{-1}$; HRMS (ESI) for $\mathrm{C}_{16} \mathrm{H}_{15} \mathrm{O}_{3} \mathrm{~N}_{2} \mathrm{~S}[\mathrm{M}-\mathrm{H}]^{-}$calcd 315.0809, found 315.0815.

HPLC ( $N$-Boc derivative): Chiralpak IC column $(250 \mathrm{~mm})$; detected at $220 \mathrm{~nm}$; hexane/i-propanol $=97 / 3$; flow $=0.7 \mathrm{~mL} / \mathrm{min}$; Retention time: $20.8 \mathrm{~min}, 25.7 \mathrm{~min}$ (major).

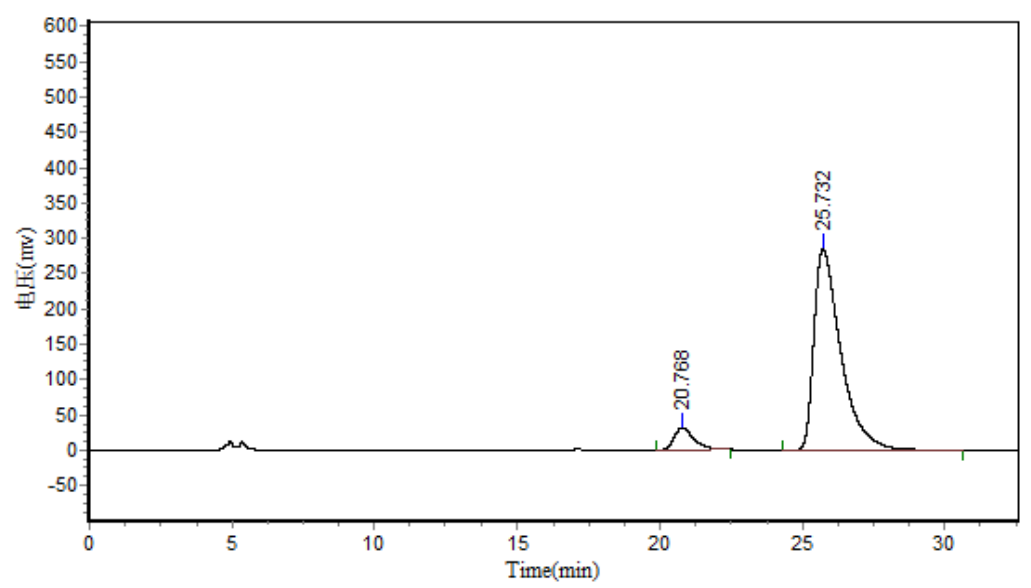

\begin{tabular}{|c|c|c|c|c|c|}
\hline \multicolumn{6}{|c|}{ Results } \\
\hline Peak No. & Peak ID & Ret Time & Height & Area & Conc. \\
\hline 1 & & 20.768 & 30872.959 & 1511848.000 & 7.5014 \\
\hline 2 & & 25.732 & 283889.500 & 18642274.000 & 92.4986 \\
\hline Total & & & 314762.459 & 20154122.000 & 100.0000 \\
\hline
\end{tabular}

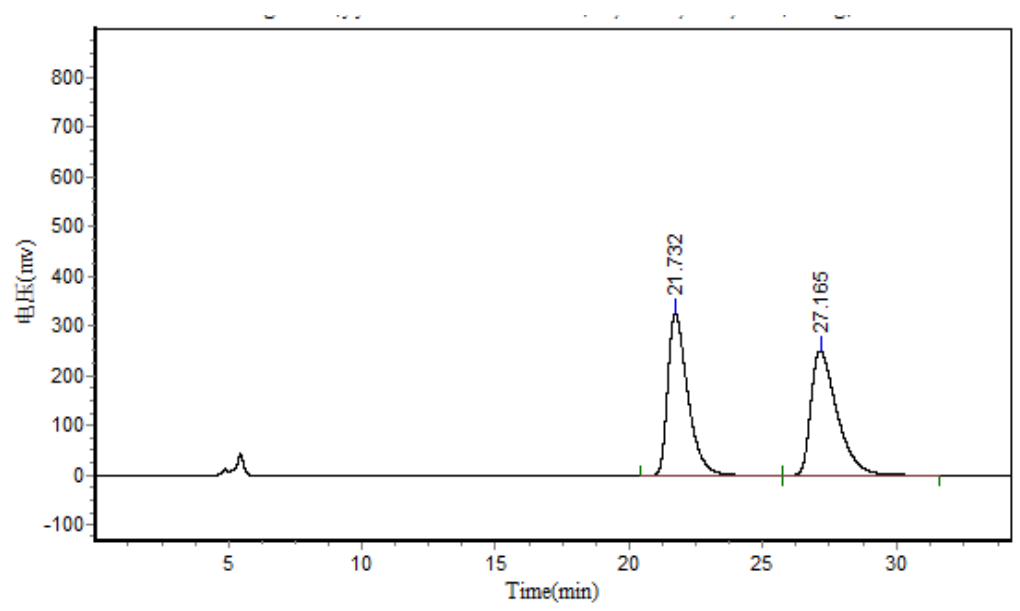

Results

\begin{tabular}{cccccc} 
Peak No. & Peak ID & Ret Time & Height & Area & Conc. \\
\hline 1 & 21.732 & 325565.500 & 17061804.000 & 49.6971 \\
2 & 27.165 & 250395.563 & 17269782.000 & 50.3029 \\
\hline Total & & 575961.063 & 34331586.000 & 100.0000
\end{tabular}


(R)-2-methyl-4-phenyl-4-(thiophen-3-yl)-1,2,5-thiadiazolidin-3-one 1,1-dioxide (3I)

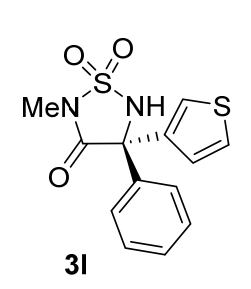

White solid, $45 \%$ yield, $98 \%$ ee; $\mathrm{mp} 103-105^{\circ} \mathrm{C}$.

$[\alpha]_{\mathrm{D}}{ }^{25}=+8.5\left(c 0.4, \mathrm{CHCl}_{3}\right) ;{ }^{1} \mathrm{H} \mathrm{NMR}\left(300 \mathrm{MHz}, \mathrm{CDCl}_{3}\right) \delta 7.71-7.57(\mathrm{~m}, 2 \mathrm{H})$, $7.40-7.27(\mathrm{~m}, 4 \mathrm{H}), 6.29(\mathrm{~d}, J=3.1 \mathrm{~Hz}, 1 \mathrm{H}), 6.21(\mathrm{~d}, J=3.3 \mathrm{~Hz}, 1 \mathrm{H}), 5.53(\mathrm{~s}$, $1 \mathrm{H}), 3.09(\mathrm{~s}, 3 \mathrm{H}) ;{ }^{13} \mathrm{C}$ NMR $\left(125 \mathrm{MHz}, \mathrm{CDCl}_{3}\right) \delta 167.8,141.4,137.9,129.8$, 129.1, 128.2, 127.8, 127.4, 127.1, 72.7, 26.5; IR (KBr) v 3439, 1729, 1632, 1417, 1337, 1185, $1012 \mathrm{~cm}^{-1}$; HRMS (ESI) for $\mathrm{C}_{13} \mathrm{H}_{11} \mathrm{O}_{3} \mathrm{~N}_{2} \mathrm{~S}_{2}[\mathrm{M}-\mathrm{H}]^{-}$calcd 307.0217, found 307.0222 .

HPLC ( $N$-Boc derivative): Chiralpak IC column $(250 \mathrm{~mm})$; detected at $254 \mathrm{~nm}$; hexane $/ i$-propanol =97/3; flow $=1.0 \mathrm{~mL} / \mathrm{min}$; Retention time: $19.4 \mathrm{~min}$ (major), $22.0 \mathrm{~min}$.

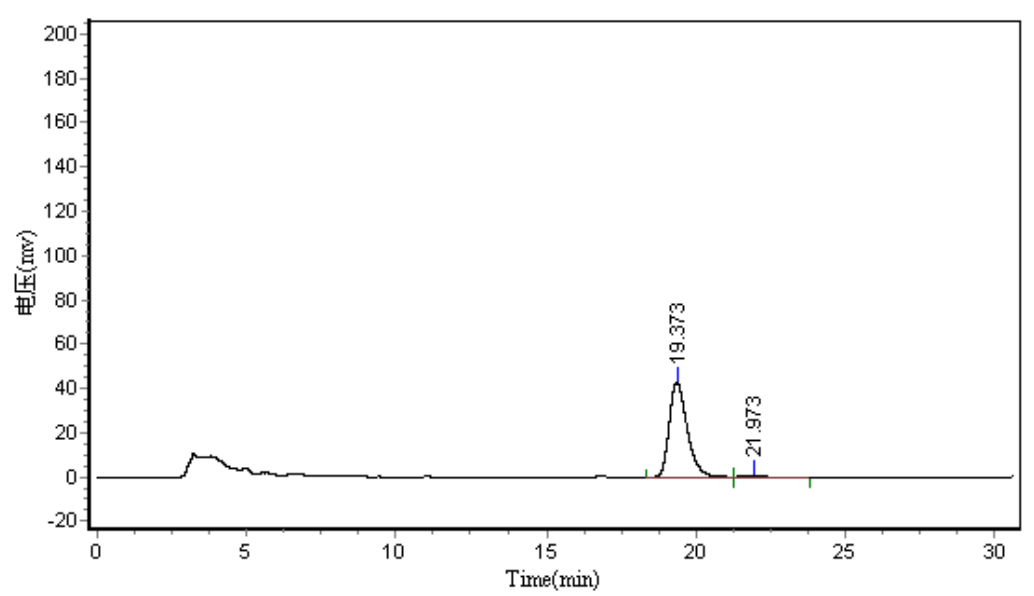

\begin{tabular}{|c|c|c|c|c|c|}
\hline \multicolumn{6}{|c|}{ Results } \\
\hline Peak No. & Peak ID & Ret Time & Height & Area & Conc. \\
\hline 1 & & 19.373 & 42925.945 & 1800459.500 & 98.9019 \\
\hline 2 & & 21.973 & 343.323 & 19990.338 & 1.0981 \\
\hline Total & & & 43269.268 & 1820449.838 & 100.0000 \\
\hline
\end{tabular}

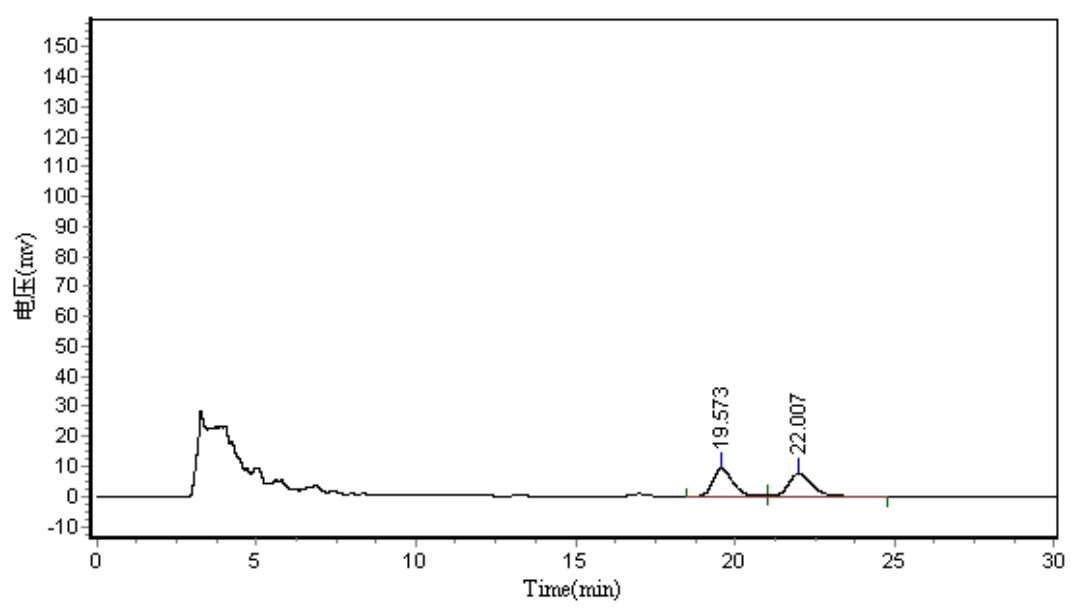

Results

\begin{tabular}{|c|c|c|c|c|c|}
\hline Peak No. & Peak ID & Ret Time & Height & Area & Conc. \\
\hline 1 & & 19.573 & 9250.647 & 396740.125 & 51.2186 \\
\hline 2 & & 22.007 & 7428.716 & 377860.875 & 48.7814 \\
\hline Total & & & 16679.363 & 774601.000 & 100.0000 \\
\hline
\end{tabular}


(R)-4-(furan-3-yl)-2-methyl-4-phenyl-1,2,5-thiadiazolidin-3-one 1,1-dioxide (3m)

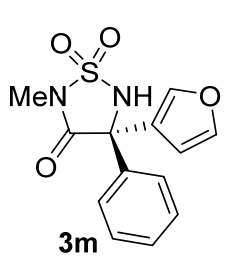

White solid, $72 \%$ yield, $98 \%$ ee; $\mathrm{mp} 120-122^{\circ} \mathrm{C}$.

$[\alpha]_{\mathrm{D}}{ }^{25}=-2.8\left(c 0.46, \mathrm{CHCl}_{3}\right) ;{ }^{1} \mathrm{H} \mathrm{NMR}\left(300 \mathrm{MHz}, \mathrm{CDCl}_{3}\right) \delta 7.71-7.57(\mathrm{~m}, 2 \mathrm{H})$, $7.40-7.27(\mathrm{~m}, 4 \mathrm{H}), 6.29$ (d, $J=3.1 \mathrm{~Hz}, 1 \mathrm{H}), 6.21(\mathrm{~d}, J=3.3 \mathrm{~Hz}, 1 \mathrm{H}), 5.53(\mathrm{~s}$, $1 \mathrm{H}), 3.09(\mathrm{~s}, 3 \mathrm{H}) ;{ }^{13} \mathrm{C}$ NMR $\left(125 \mathrm{MHz}, \mathrm{CDCl}_{3}\right) \delta 166.2,149.5,144.4,134.5$, 129.6, 128.8, 127.1, 112.5, 111.1, 70.9, 26.3; IR (KBr) v 3431, 1724, 1648, 1420, 1333, $1179 \mathrm{~cm}^{-1}$; HRMS (ESI) for $\mathrm{C}_{13} \mathrm{H}_{11} \mathrm{O}_{4} \mathrm{~N}_{2} \mathrm{~S}$ [M-H] calcd 291.0445, found 291.0451.

HPLC ( $N$-Boc derivative): Chiralpak IC column $(250 \mathrm{~mm})$; detected at $220 \mathrm{~nm}$; hexane/i-propanol $=97 / 3$; flow $=0.7 \mathrm{~mL} / \mathrm{min}$; Retention time: $29.3 \mathrm{~min}$ (major), $33.0 \mathrm{~min}$.

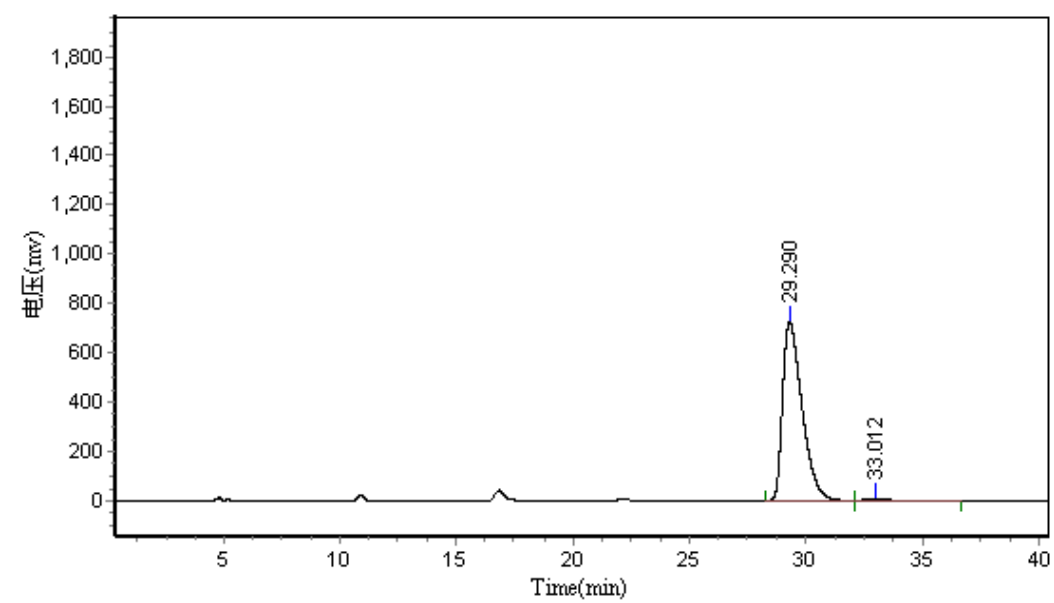

Results

\begin{tabular}{|c|c|c|c|c|c|}
\hline Peak No. & Peak ID & Ret Time & Height & Area & Conc. \\
\hline 1 & & 29.290 & 727032.125 & 42307708.000 & 98.8324 \\
\hline 2 & & 33.012 & 6929.660 & 499817.438 & 1.1676 \\
\hline Total & & & 733961.785 & 42807525.438 & 100.0000 \\
\hline
\end{tabular}

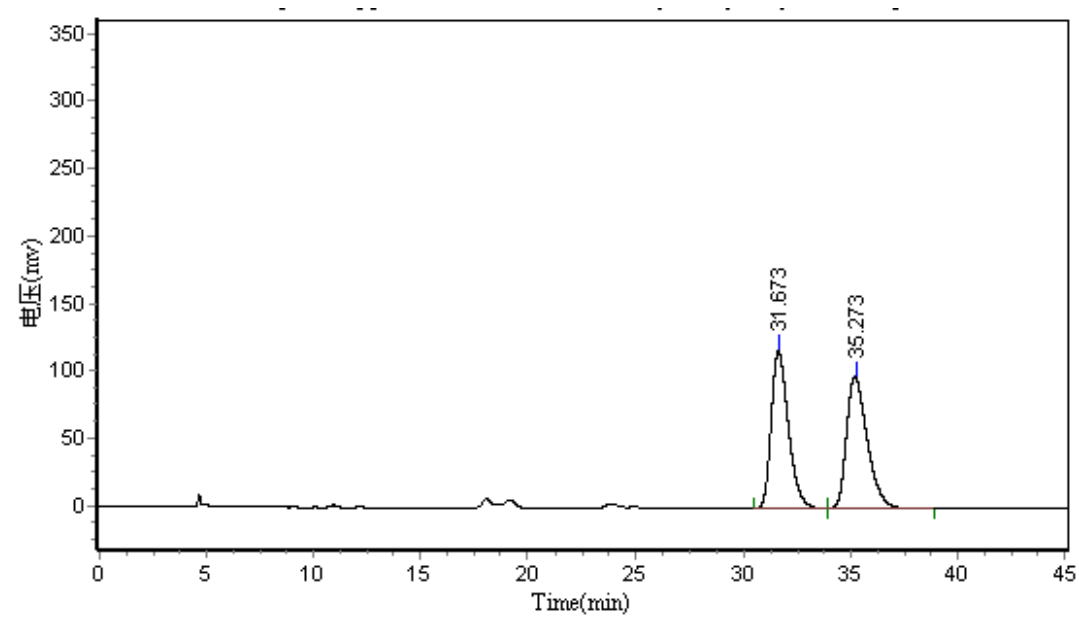

\begin{tabular}{|c|c|c|c|c|c|}
\hline \multicolumn{6}{|c|}{ Results } \\
\hline Peak No. & Peak ID & Ret Time & Height & Area & Conc. \\
\hline 1 & & 31.673 & 116769.164 & 6574740.000 & 49.6534 \\
\hline 2 & & 35.273 & 97589.133 & 6666524.500 & 50.3466 \\
\hline Total & & & 214358.297 & 13241264.500 & 100.0000 \\
\hline
\end{tabular}




\section{(S,E)-2-methyl-4-phenyl-4-styryl-1,2,5-thiadiazolidin-3-one 1,1-dioxide (3n)}<smiles>CN1C(=O)[C@](/C=C/c2ccccc2)(c2ccccc2)NS1(=O)=O</smiles>

$3 n$

White solid, $91 \%$ yield, $66 \%$ ee; $\mathrm{mp} 150-157^{\circ} \mathrm{C}$.

$[\alpha]_{\mathrm{D}}{ }^{25}=+19.8\left(c 0.555, \mathrm{CHCl}_{3}\right) ;{ }^{1} \mathrm{H}$ NMR $\left(400 \mathrm{MHz}, \mathrm{CDCl}_{3}\right) \delta 7.57-7.50$

$(\mathrm{m}, 2 \mathrm{H}), 7.41(\mathrm{dd}, J=8.8,4.0 \mathrm{~Hz}, 5 \mathrm{H}), 7.37-7.26(\mathrm{~m}, 3 \mathrm{H}), 6.96(\mathrm{~d}, J=15.8$ $\mathrm{Hz}, 1 \mathrm{H}), 6.51(\mathrm{~d}, J=15.8 \mathrm{~Hz}, 1 \mathrm{H}), 5.21(\mathrm{~s}, 1 \mathrm{H}), 3.17(\mathrm{~s}, 3 \mathrm{H}) ;{ }^{13} \mathrm{C}$ NMR $(126$ $\left.\mathrm{MHz}, \mathrm{CDCl}_{3}\right) \delta 168.2,137.1,135.1,134.2,129.6,129.3,128.93,128.86$, 127.2, 127.0, 125.1, 73.6, 26.2; IR (KBr) v 3451, 3240, 1708, 1388, 1337, 1185, 991, 746, $691 \mathrm{~cm}^{-1}$; HRMS (ESI) for $\mathrm{C}_{17} \mathrm{H}_{17} \mathrm{O}_{3} \mathrm{~N}_{2} \mathrm{~S}[\mathrm{M}+\mathrm{H}]^{-}$calcd 329.0954, found 329.0959 .

HPLC (N-Boc derivative): Chiralpak IC column (250 mm); detected at $220 \mathrm{~nm}$; hexane/i-propanol $=99 / 1$; flow $=0.7 \mathrm{~mL} / \mathrm{min}$; Retention time: $30.5 \mathrm{~min}, 37.1 \mathrm{~min}$ (major).

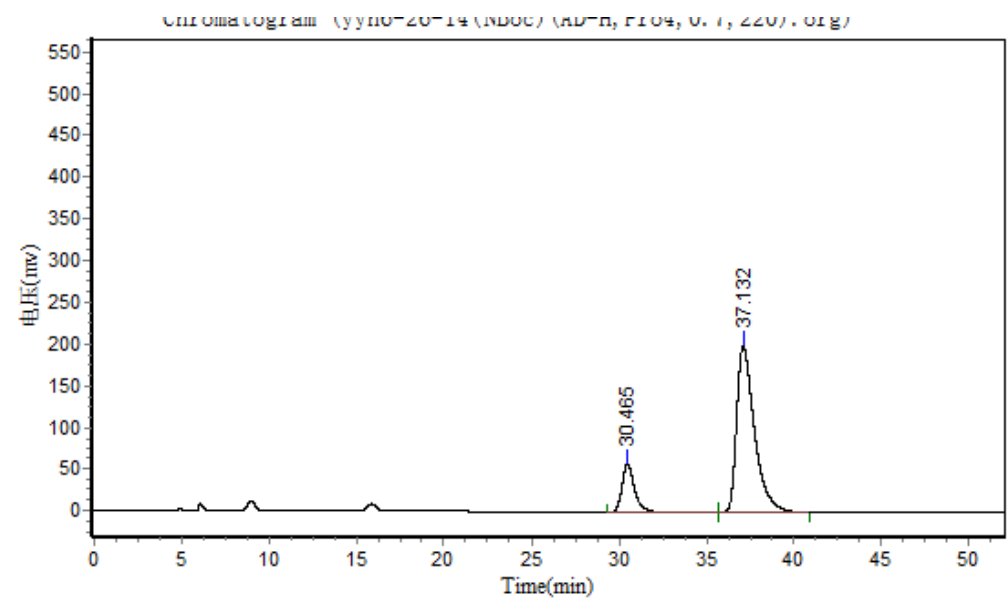

Results

\begin{tabular}{|c|c|c|c|c|c|}
\hline Peak No. & Peak ID & Ret Time & Height & Area & Conc. \\
\hline 1 & & 30.465 & 57947.223 & 2937453.750 & 17.0800 \\
\hline 2 & & 37.132 & 199641.484 & 14260803.000 & 82.9201 \\
\hline Total & & & 257588.707 & 17198256.750 & 100.0000 \\
\hline
\end{tabular}

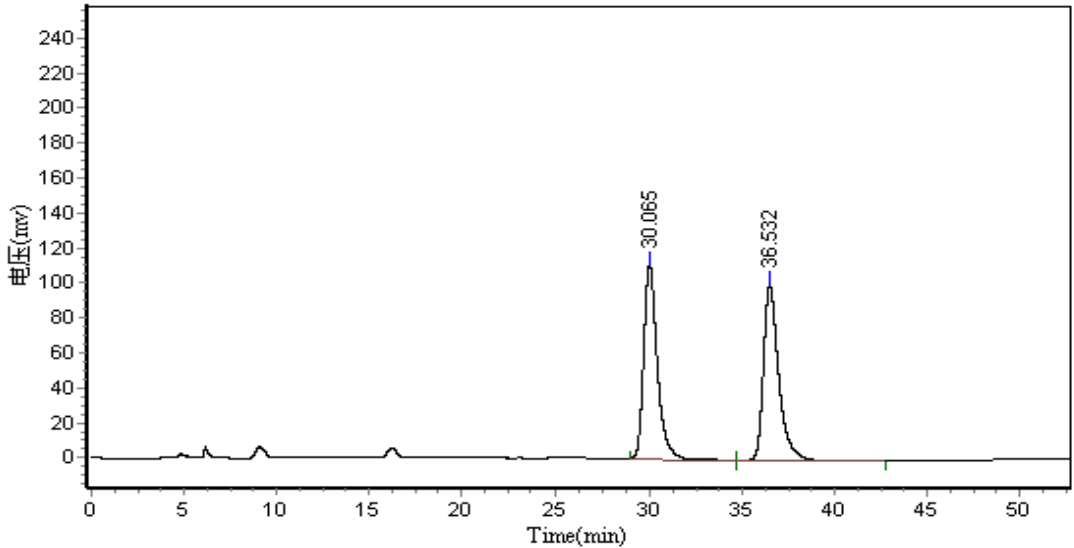

Results

\begin{tabular}{|c|c|c|c|c|c|}
\hline Peak No. & Peak ID & Ret Time & Height & Area & Conc. \\
\hline$\overline{1}$ & & 30.065 & 111538.750 & 5775746.500 & 50.1026 \\
\hline 2 & & 36.532 & 100058.156 & 5752091.500 & 49.8974 \\
\hline Total & & & 211596.906 & 11527838.000 & 100.0000 \\
\hline
\end{tabular}


(S)-2-methyl-4-phenyl-4-(p-tolyl)-1,2,5-thiadiazolidin-3-one 1,1-dioxide (3a')<smiles>Cc1ccc(C2(c3ccccc3)NS(=O)(=O)[C@H](C)C2=O)cc1</smiles>

White solid, 96\% yield, $94 \%$ ee; mp $107-109^{\circ} \mathrm{C}$.

$[\alpha]_{\mathrm{D}}{ }^{25}=-10.7\left(c 0.44, \mathrm{CHCl}_{3}\right) ;{ }^{1} \mathrm{H} \mathrm{NMR}\left(300 \mathrm{MHz}, \mathrm{CDCl}_{3}\right) \delta 7.53-7.44(\mathrm{~m}$, 2H), $7.41-7.35(\mathrm{~m}, 3 \mathrm{H}), 7.32(\mathrm{~d}, J=8.3 \mathrm{~Hz}, 2 \mathrm{H}), 7.18(\mathrm{~d}, J=8.0 \mathrm{~Hz}, 2 \mathrm{H})$, $5.06(\mathrm{~s}, 1 \mathrm{H}), 3.19(\mathrm{~s}, 3 \mathrm{H}), 2.35(\mathrm{~s}, 3 \mathrm{H}) ;{ }^{13} \mathrm{C} \mathrm{NMR}\left(125 \mathrm{MHz}, \mathrm{CDCl}_{3}\right) \delta 168.9$, $139.5,138.2,135.5,129.8,129.3,129.0,127.7,127.6,75.6,26.4,21.3$; IR (KBr) $v$ 3185, 1710, 1460, 1405, 1334, 1180, 1071, $970 \mathrm{~cm}^{-1}$; HRMS (ESI) for $\mathrm{C}_{16} \mathrm{H}_{17} \mathrm{~N}_{2} \mathrm{O}_{3} \mathrm{~S}[\mathrm{M}+\mathrm{H}]^{+}$calcd 317.0954, found 317.0959.

HPLC ( $N$-Me derivative): Chiralpak IC column $(250 \mathrm{~mm})$; detected at $220 \mathrm{~nm}$; hexane/i-propanol =97/3; flow $=0.7 \mathrm{~mL} / \mathrm{min}$; Retention time: $22.5 \mathrm{~min}, 24.5 \mathrm{~min}$ (major).

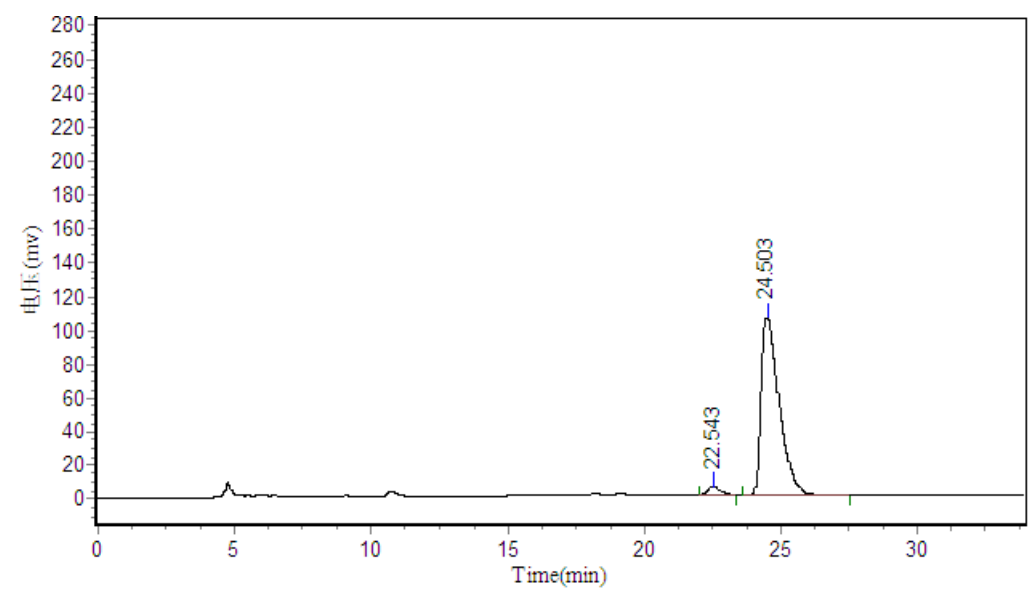

Results

\begin{tabular}{cccccc} 
Peak No. & Peak I & Ret Time & Height & Area & Conc. \\
\hline 1 & 22.543 & 4755.738 & 161939.594 & 3.2490 \\
2 & 24.503 & 104848.109 & 4822418.000 & 96.7510 \\
\hline Total & & 109603.848 & 4984357.594 & 100.0000
\end{tabular}

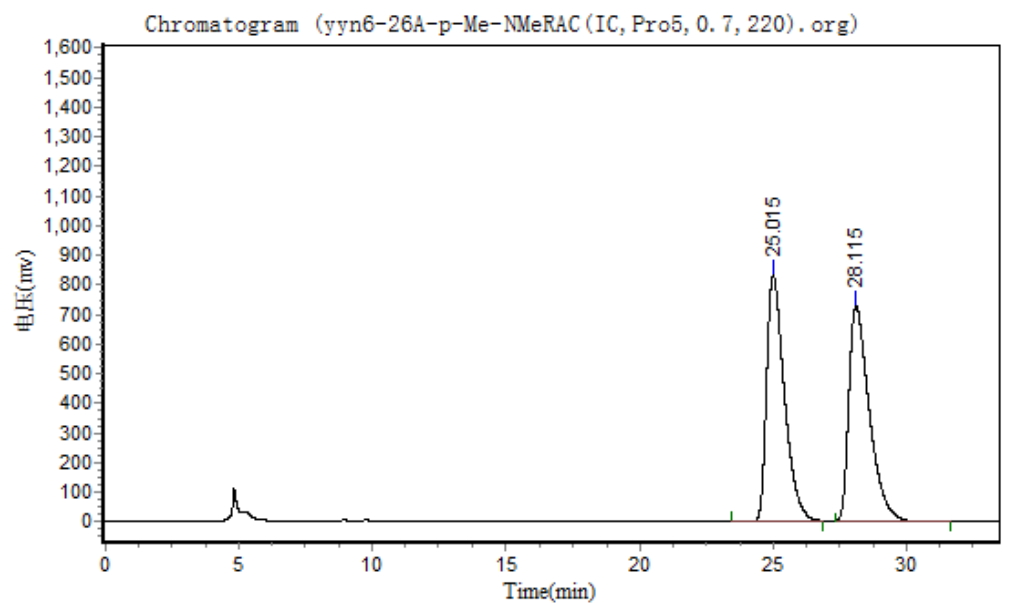

\begin{tabular}{|c|c|c|c|c|c|}
\hline \multicolumn{6}{|c|}{ Results } \\
\hline Peak No. & Peak ID & Ret Time & Height & Area & Conc. \\
\hline 1 & & 25.015 & 831891.625 & 38836504.000 & 49.8222 \\
\hline 2 & & 28.115 & 728628.938 & 39113720.000 & 50.1778 \\
\hline Total & & & 1560520.563 & 77950224.000 & 100.0000 \\
\hline
\end{tabular}


(R)-4-(4-chlorophenyl)-2-methyl-4-(p-tolyl)-1,2,5-thiadiazolidin-3-one 1,1-dioxide (3o)<smiles>Cc1ccc(C2(c3ccc(Cl)cc3)NS(=O)(=O)[C@H](C)C2=O)cc1</smiles>

White solid, $95 \%$ yield, $95 \%$ ee; $\mathrm{mp} 138-140{ }^{\circ} \mathrm{C}$.

$[\alpha]_{\mathrm{D}}{ }^{25}=-51.9\left(c 0.465, \mathrm{CHCl}_{3}\right) ;{ }^{1} \mathrm{H}$ NMR $\left(300 \mathrm{MHz}, \mathrm{CDCl}_{3}\right) \delta 7.51(\mathrm{~d}, J=$ $8.8 \mathrm{~Hz}, 2 \mathrm{H}), 7.39-7.32(\mathrm{~m}, 2 \mathrm{H}), 7.19(\mathrm{~d}, J=3.3 \mathrm{~Hz}, 4 \mathrm{H}), 5.06(\mathrm{~s}, 1 \mathrm{H})$, $3.19(\mathrm{~s}, 3 \mathrm{H}), 2.35(\mathrm{~s}, 3 \mathrm{H}) ;{ }^{13} \mathrm{C} \mathrm{NMR}\left(125 \mathrm{MHz}, \mathrm{CDCl}_{3}\right) \delta 168.6,139.9$, 136.2, 135.6, 135.5, 130.0, 129.2, 129.1, 127.5, 75.0, 26.5, 21.3; IR (KBr) $v$ 3438, 3185, 1711, 1489, 1411, 1339, 1212, 1186, 1091, 1071, 1013, 969 $\mathrm{cm}^{-1}$; HRMS (ESI) for $\mathrm{C}_{16} \mathrm{H}_{16} \mathrm{O}_{3} \mathrm{~N}_{2} \mathrm{ClS}[\mathrm{M}+\mathrm{H}]^{+}$calcd 351.0565, found 351.0558 .

HPLC ( $N$-Boc derivative): Chiralpak IC column $(250 \mathrm{~mm})$; detected at $220 \mathrm{~nm}$; hexane/i-propanol $=97 / 3$; flow $=0.7 \mathrm{~mL} / \mathrm{min}$; Retention time: $13.8 \mathrm{~min}, 15.1 \mathrm{~min}$ (major)

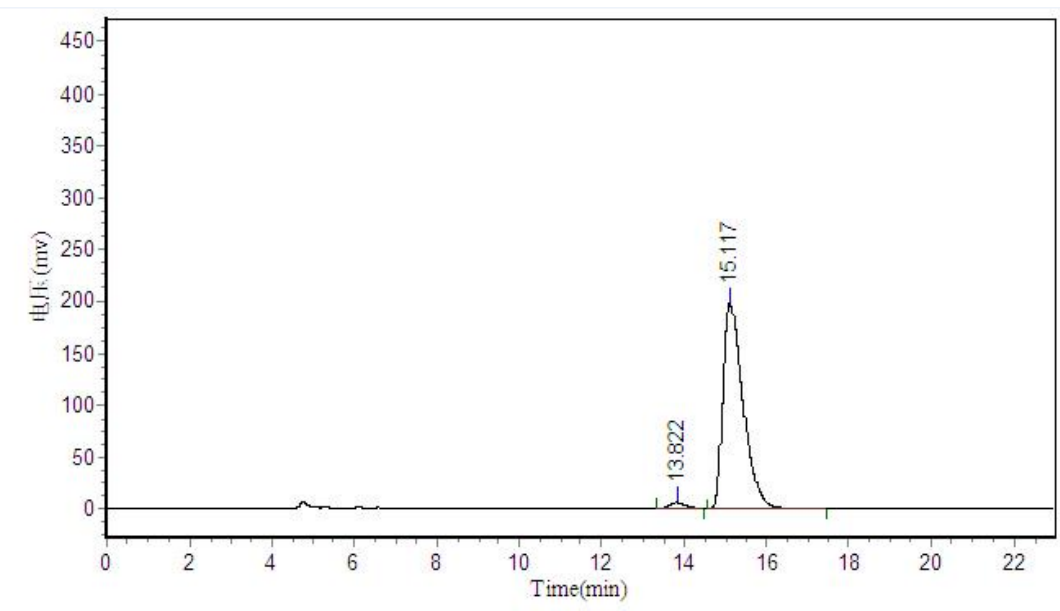

Results

\begin{tabular}{|c|c|c|c|c|c|}
\hline Peak No. & Peak ID & Ret Time & Height & Area & Conc. \\
\hline 1 & & 13.822 & 6589.772 & 172675.094 & 2.4666 \\
\hline 2 & & 15.117 & 198433.766 & 6827878.000 & 97.5334 \\
\hline Total & & & 205023.538 & 7000553.094 & 100.0000 \\
\hline
\end{tabular}

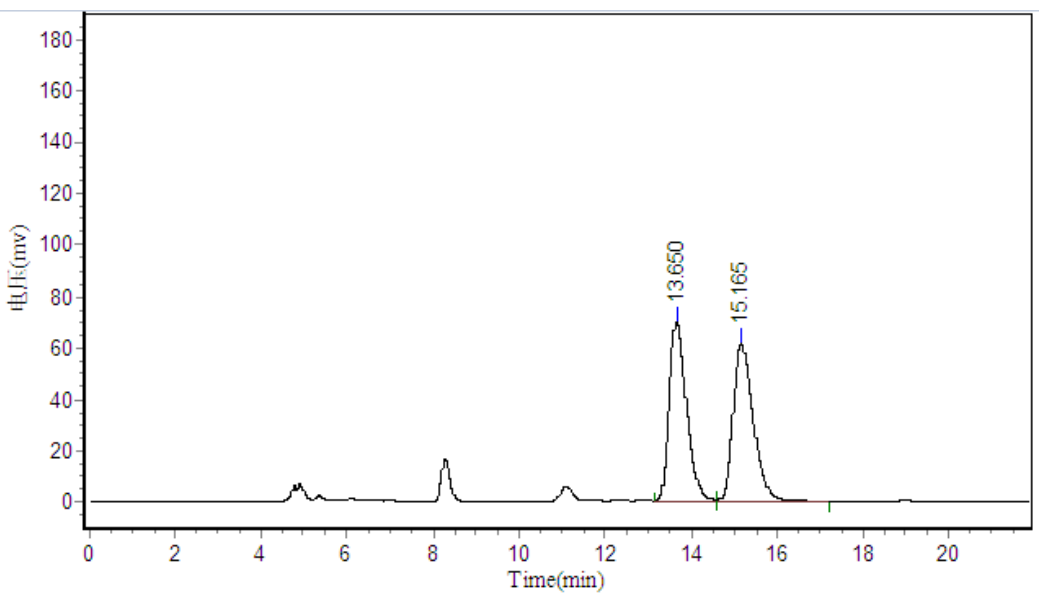

Results

\begin{tabular}{|c|c|c|c|c|c|}
\hline Peak No. & Peak ID & Ret Time & Height & Area & Conc. \\
\hline 1 & & 13.650 & 69660.328 & 1974518.125 & 49.5292 \\
\hline 2 & & 15.165 & 61286.012 & 2012059.625 & 50.4708 \\
\hline Total & & & 130946.340 & 3986577.750 & 100.0000 \\
\hline
\end{tabular}


(R)-4-(4-fluorophenyl)-2-methyl-4-(p-tolyl)-1,2,5-thiadiazolidin-3-one 1,1-dioxide (3p)<smiles>Cc1ccc(C(NS(=O)(=O)[O-])c2ccc(F)cc2)cc1</smiles>

White solid, 94\% yield, 94\% ee; $\mathrm{mp} 129-131{ }^{\circ} \mathrm{C}$.

$[\alpha]_{\mathrm{D}}{ }^{25}=-40.8\left(c 0.56, \mathrm{CHCl}_{3}\right) ;{ }^{1} \mathrm{H} \mathrm{NMR}\left(300 \mathrm{MHz}, \mathrm{CDCl}_{3}\right) \delta 7.53(\mathrm{dd}, J=$ 8.7, $5.2 \mathrm{~Hz}, 2 \mathrm{H}), 7.24(\mathrm{~d}, J=8.3 \mathrm{~Hz}, 2 \mathrm{H}), 7.18(\mathrm{~d}, J=8.2 \mathrm{~Hz}, 2 \mathrm{H}), 7.07$ (t, $J$ $=8.6 \mathrm{~Hz}, 2 \mathrm{H}), 5.06(\mathrm{~s}, 1 \mathrm{H}), 3.19(\mathrm{~s}, 3 \mathrm{H}), 2.36(\mathrm{~s}, 3 \mathrm{H}),{ }^{13} \mathrm{C}$ NMR $(125 \mathrm{MHz}$, $\left.\mathrm{CDCl}_{3}\right) \delta 168.8,163.2\left(\mathrm{~d},{ }^{1} J_{\mathrm{CF}}=248.8 \mathrm{~Hz}\right), 139.8,135.7,133.7,130.0,129.8$ $\left(\mathrm{d},{ }^{3} J_{\mathrm{CF}}=8.8 \mathrm{~Hz}\right), 127.5,115.9\left(\mathrm{~d},{ }^{2} J_{\mathrm{CF}}=22.5 \mathrm{~Hz}\right), 115.8,75.1,26.4,21.3$; ${ }^{19} \mathrm{~F}$ NMR $\left(471 \mathrm{MHz}, \mathrm{CDCl}_{3}\right) \delta-112.3(\mathrm{~s})$; IR (KBr) $v$ 3428, 3183, 1710, 1605, 1506, 1413, 1341, 1226, 1185, 1159, 1071, $970 \mathrm{~cm}^{-1}$; HRMS (ESI) for $\mathrm{C}_{16} \mathrm{H}_{16} \mathrm{O}_{3} \mathrm{~N}_{2} \mathrm{FS}[\mathrm{M}+\mathrm{H}]^{+}$calcd 335.0860, found 335.0856 .

HPLC ( $N$-Boc derivative): Chiralpak IC column $(250 \mathrm{~mm})$; detected at $220 \mathrm{~nm}$; hexane/ $i$-propanol $=97 / 3$; flow $=0.7 \mathrm{~mL} / \mathrm{min}$; Retention time: $14.0 \mathrm{~min}, 15.7 \mathrm{~min}$ (major)

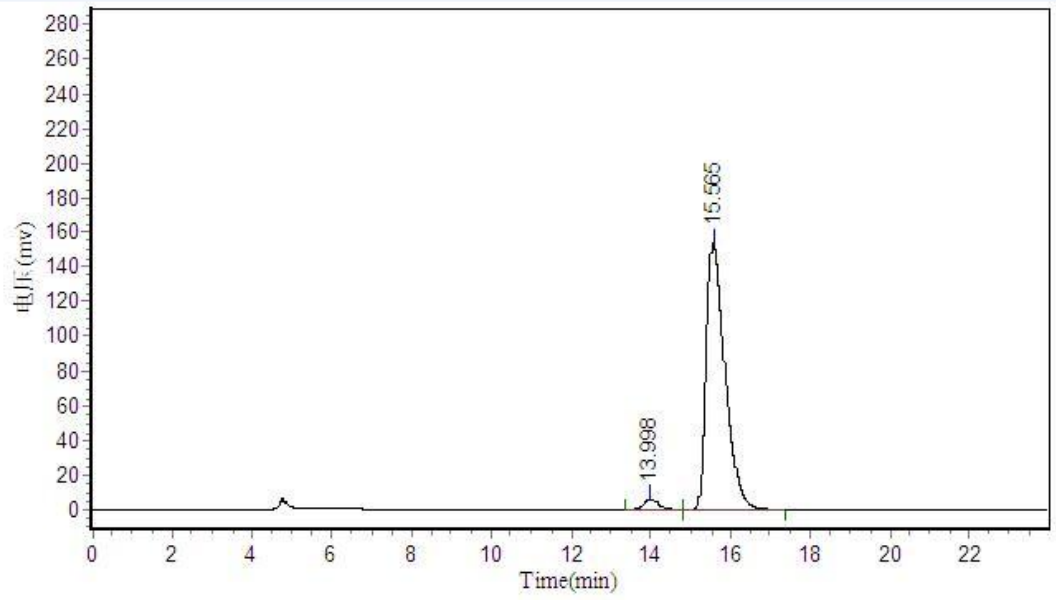

\begin{tabular}{|c|c|c|c|c|c|}
\hline \multicolumn{6}{|c|}{ Results } \\
\hline Peak No. & Peak ID & Ret Time & Height & Area & Conc. \\
\hline 1 & & 13.998 & 5842.487 & 162080.813 & 3.0880 \\
\hline 2 & & 15.565 & 152722.688 & 5086614.000 & 96.9120 \\
\hline Total & & & 158565.175 & 5248694.813 & 100.0000 \\
\hline
\end{tabular}

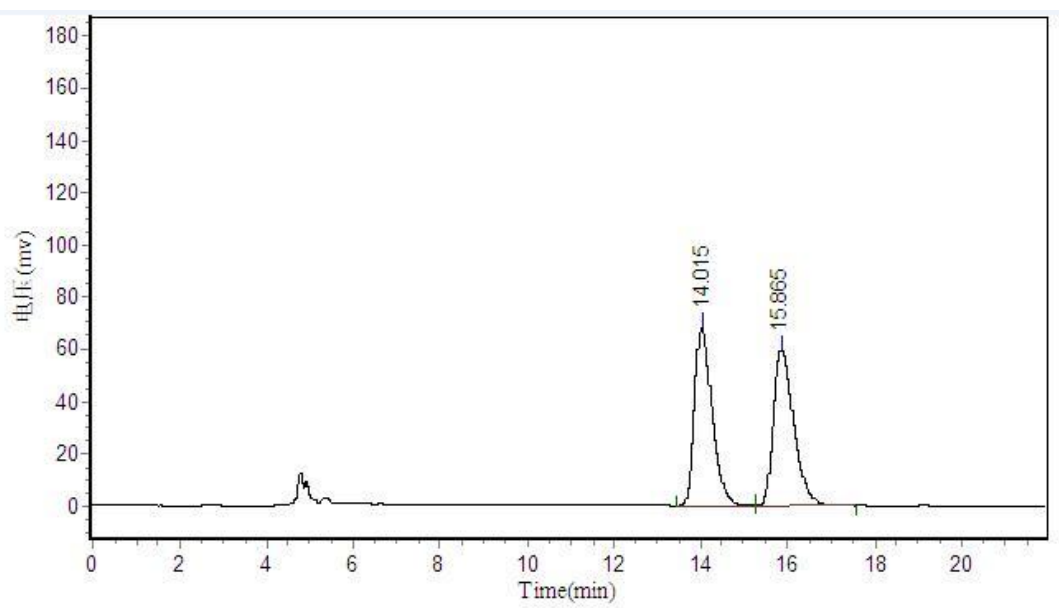

\begin{tabular}{|c|c|c|c|c|c|}
\hline \multicolumn{6}{|c|}{ Results } \\
\hline Peak No. & Peak ID & Ret Time & Height & Area & Conc. \\
\hline 1 & & 14.015 & 67825.938 & 1929191.500 & 49.7894 \\
\hline 2 & & 15.865 & 59565.824 & 1945513.500 & 50.2106 \\
\hline Total & & & 127391.762 & 3874705.000 & 100.0000 \\
\hline
\end{tabular}


(S)-4-(4-chlorophenyl)-2-methyl-4-phenyl-1,2,5-thiadiazolidin-3-one 1,1-dioxide (3b')<smiles>CNC(=O)C(c1ccccc1)(c1ccc(Cl)cc1)S(=O)(=O)[O-]</smiles>

White solid, 98\% yield, $96 \%$ ee; $\mathrm{mp} 134-136^{\circ} \mathrm{C}$.

$[\alpha]_{\mathrm{D}}{ }^{25}=+44.3\left(c 0.435, \mathrm{CHCl}_{3}\right) ;{ }^{1} \mathrm{H}$ NMR $\left(300 \mathrm{MHz}, \mathrm{CDCl}_{3}\right) \delta 7.49(\mathrm{~d}, J=8.7$ $\mathrm{Hz}, 2 \mathrm{H}), 7.44-7.30(\mathrm{~m}, 7 \mathrm{H}), 5.13(\mathrm{~s}, 1 \mathrm{H}), 3.19(\mathrm{~s}, 3 \mathrm{H}) ;{ }^{13} \mathrm{C}$ NMR $(125 \mathrm{MHz}$, $\left.\mathrm{CDCl}_{3}\right) \delta 168.4,138.4,136.2,135.6,129.7,129.3,129.2,129.1,127.6,75.1$, 26.5; IR (KBr) v 3431, 3184, 1712, 1491, 1448, 1420, 1342, 1218, 1180, 1092, 1068, 1014, $970 \mathrm{~cm}^{-1}$; HRMS (ESI) for $\mathrm{C}_{15} \mathrm{H}_{14} \mathrm{O}_{3} \mathrm{~N}_{2} \mathrm{ClS}[\mathrm{M}+\mathrm{H}]^{+}$calcd 337.0408, found 337.0404.

HPLC: Chiralpak AY-H column $(250 \mathrm{~mm})$; detected at $220 \mathrm{~nm}$; hexane $/ i$-propanol $=80 / 20$; flow $=$ $1 \mathrm{~mL} / \mathrm{min}$; Retention time: $41.5 \mathrm{~min}$ (major), $67.5 \mathrm{~min}$.

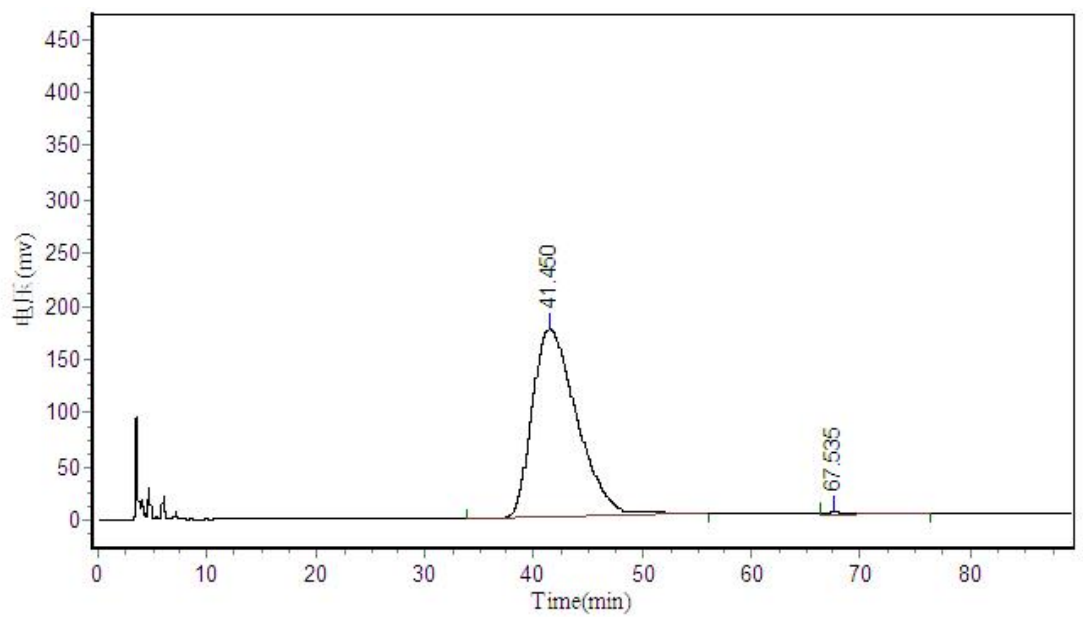

Results

\begin{tabular}{cccccc} 
Peak No. & Peak D & Ret Time & Height & Area & Conc. \\
\hline 1 & 41.450 & 175796.688 & 49973480.000 & 98.3158 \\
2 & 67.535 & 2673.069 & 856092.313 & 1.6842 & 100.0000
\end{tabular}

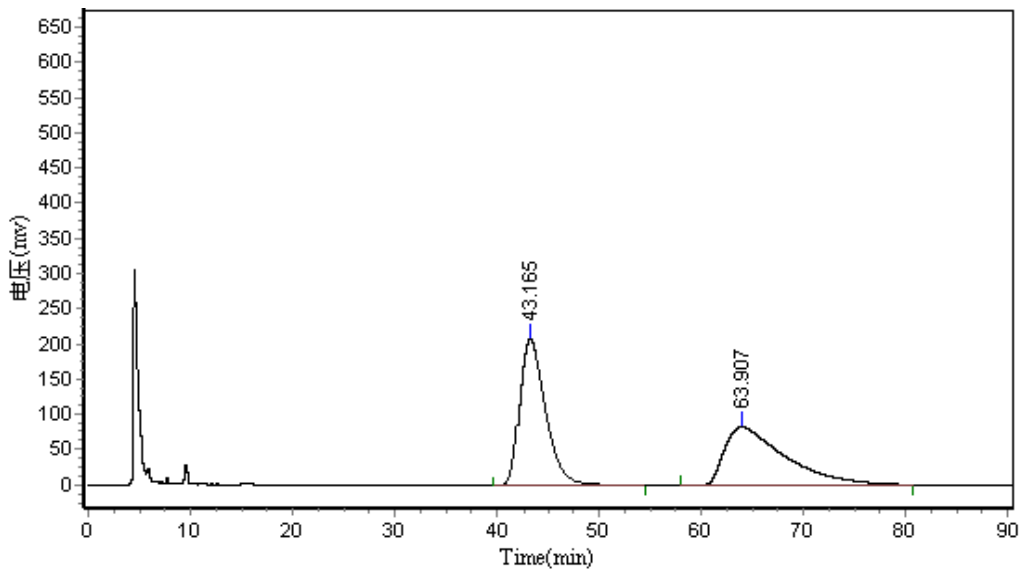

Results

\begin{tabular}{cccccc} 
Peak No. & Peak ID & Ret Time & Height & Area & Conc. \\
\hline 1 & 43.165 & 208089.063 & 36603732.000 & 51.3001 \\
2 & 63.907 & 83746.961 & 34748368.000 & 48.6999 \\
\hline Total & & 291836.023 & 71352100.000 & 100.0000
\end{tabular}


(S)-4-(4-chlorophenyl)-2-methyl-4-(p-tolyl)-1,2,5-thiadiazolidin-3-one 1,1-dioxide (3o')<smiles>Cc1ccc(C(NS(C)(=O)=O)C2NS(=O)(=O)NC2=O)cc1</smiles>

White solid, $98 \%$ yield, $96 \%$ ee; $\mathrm{mp} 138-141{ }^{\circ} \mathrm{C}$.

$[\alpha]_{\mathrm{D}}{ }^{25}=+47.2\left(c 0.505, \mathrm{CHCl}_{3}\right) ;{ }^{1} \mathrm{H}$ NMR $\left(300 \mathrm{MHz}, \mathrm{CDCl}_{3}\right) \delta 7.51(\mathrm{~d}, J=8.8$

$\mathrm{Hz}, 2 \mathrm{H}), 7.39-7.33(\mathrm{~m}, 2 \mathrm{H}), 7.20$ (d, $J=3.3 \mathrm{~Hz}, 4 \mathrm{H}), 5.07$ (s, 1H), 3.19 (s, $3 \mathrm{H}), 2.36(\mathrm{~s}, 3 \mathrm{H}) ;{ }^{13} \mathrm{C} \mathrm{NMR}\left(100 \mathrm{MHz}, \mathrm{CDCl}_{3}\right) \delta 168.6,139.9,136.2,135.6$, $135.5,130.0,129.2,129.1,127.5,75.0,26.5,21.3$; IR (KBr) v 3440, 3185, 1712, 1490, 1411, 1338, 1212, 1186, 1091, 1071, 1013, $970 \mathrm{~cm}^{-1}$; HRMS (ESI) for $\mathrm{C}_{16} \mathrm{H}_{16} \mathrm{O}_{3} \mathrm{~N}_{2} \mathrm{ClS}[\mathrm{M}+\mathrm{H}]^{+}$calcd 351.0565, found 351.0561 .

HPLC ( $N$-Boc derivative): Chiralpak IC column $(250 \mathrm{~mm})$; detected at $220 \mathrm{~nm}$; hexane $/ i$-propanol $=97 / 3$; flow $=0.7 \mathrm{~mL} / \mathrm{min}$; Retention time: $13.6 \mathrm{~min}$ (major), $15.6 \mathrm{~min}$.

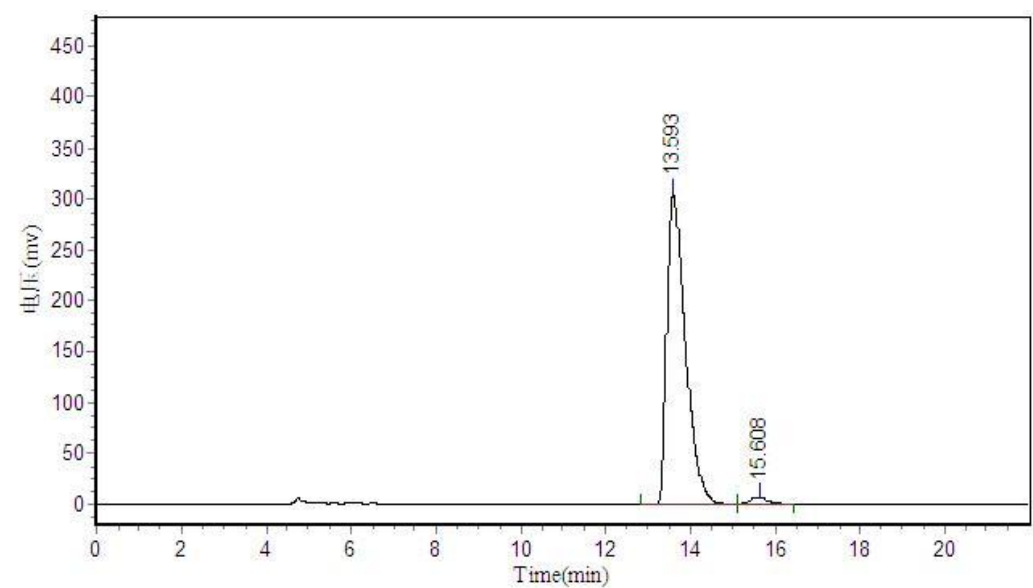

\begin{tabular}{|c|c|c|c|c|c|}
\hline \multicolumn{6}{|c|}{ Results } \\
\hline Peak No. & Peak ID & Ret Time & Height & Area & Conc. \\
\hline 1 & & 13.593 & 304857.063 & 9263543.000 & 97.8800 \\
\hline 2 & & 15.608 & 6143.436 & 200642.578 & 2.1200 \\
\hline Total & & & 311000.498 & 9464185.578 & 100.0000 \\
\hline
\end{tabular}

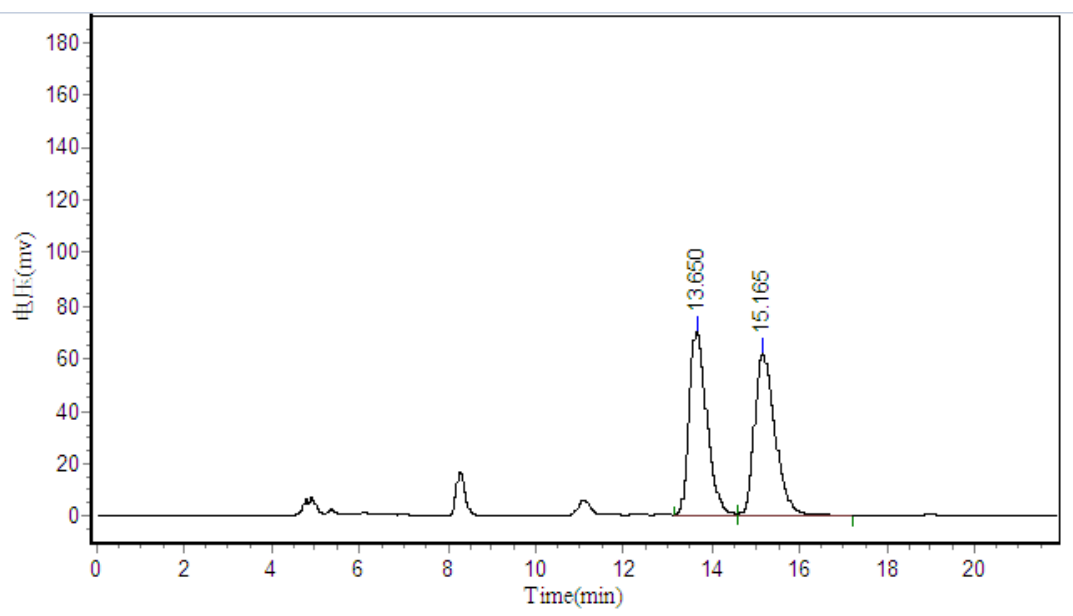

Results

\begin{tabular}{|c|c|c|c|c|c|}
\hline Peak No. & Peak ID & Ret Time & Height & Area & Conc. \\
\hline 1 & & 13.650 & 69660.328 & 1974518.125 & 49.5292 \\
\hline 2 & & 15.165 & 61286.012 & 2012059.625 & 50.4708 \\
\hline Tota 1 & & & 130946.340 & 3986577.750 & 100.0000 \\
\hline
\end{tabular}


(S)-4-(4-fluorophenyl)-2-methyl-4-phenyl-1,2,5-thiadiazolidin-3-one 1,1-dioxide (3d')

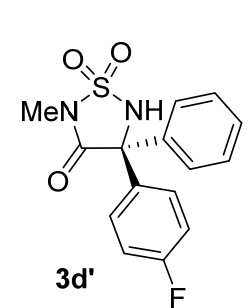

White solid, $94 \%$ yield, $97 \%$ ee; $\mathrm{mp} 143-145^{\circ} \mathrm{C}$.

$[\alpha]_{\mathrm{D}}{ }^{25}=+33.3\left(c 0.48, \mathrm{CHCl}_{3}\right) ;{ }^{1} \mathrm{H} \mathrm{NMR}\left(300 \mathrm{MHz}, \mathrm{CDCl}_{3}\right) \delta 7.51(\mathrm{dd}, J=9.1$, $5.1 \mathrm{~Hz}, 2 \mathrm{H}), 7.38(\mathrm{~s}, 5 \mathrm{H}), 7.07(\mathrm{t}, J=8.7 \mathrm{~Hz}, 2 \mathrm{H}), 5.11(\mathrm{~s}, 1 \mathrm{H}), 3.20(\mathrm{~s}, 3 \mathrm{H}) ;{ }^{13} \mathrm{C}$ NMR $\left(125 \mathrm{MHz}, \mathrm{CDCl}_{3}\right) \delta 168.6,163.2\left(\mathrm{~d},{ }^{1} J_{\mathrm{CF}}=248.8 \mathrm{~Hz}\right), 138.5,133.7$, $129.8\left(\mathrm{~d},{ }^{3} J_{\mathrm{CF}}=8.4 \mathrm{~Hz}\right), 129.7,129.3,127.6,116.0\left(\mathrm{~d},{ }^{2} J_{\mathrm{CF}}=21.3 \mathrm{~Hz}\right), 75.1$, 26.5; ${ }^{19} \mathrm{~F}$ NMR $\left(471 \mathrm{MHz}, \mathrm{CDCl}_{3}\right) \delta-112.1(\mathrm{~s})$; IR (KBr) $v 3433,3181,1713$, $1606,1508,1410,1342,1225,1187,1164,1070,969 \mathrm{~cm}^{-1}$; HRMS (ESI) for $\mathrm{C}_{15} \mathrm{H}_{14} \mathrm{O}_{3} \mathrm{~N}_{2} \mathrm{FS}$ $[\mathrm{M}+\mathrm{H}]^{+}$calcd 321.0704, found 321.0706.

HPLC ( $N$-Boc derivative): Chiralpak IC column $(250 \mathrm{~mm})$; detected at $220 \mathrm{~nm}$; hexane $/ i$-propanol $=97 / 3$; flow $=0.7 \mathrm{~mL} / \mathrm{min}$; Retention time: $12.9 \mathrm{~min}$ (major), 16.0 .

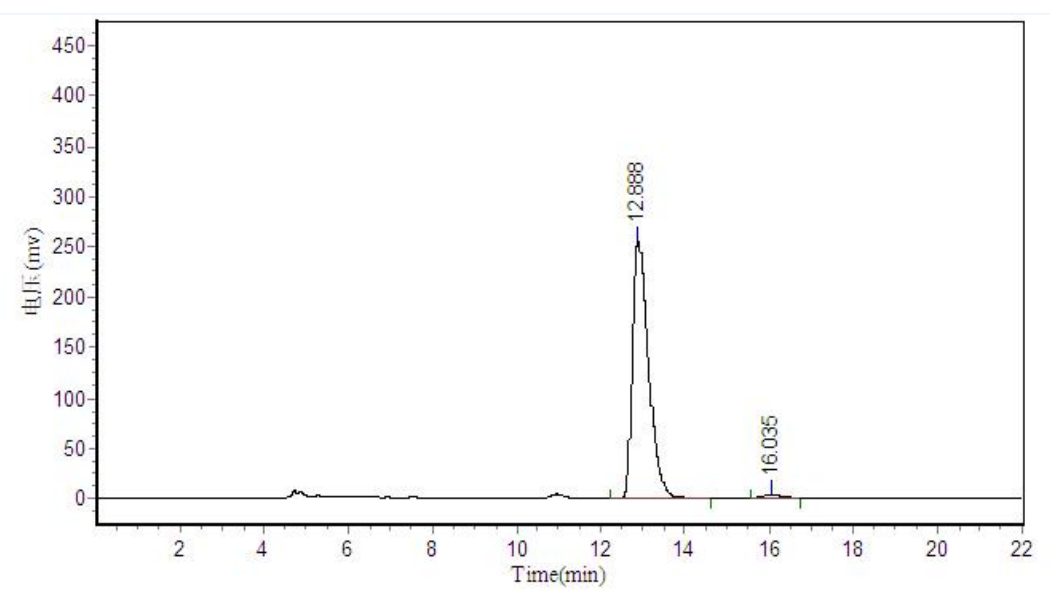

\begin{tabular}{|c|c|c|c|c|c|}
\hline \multicolumn{6}{|c|}{ Results } \\
\hline Peak No. & Peak ID & Ret Time & Height & Area & Conc. \\
\hline 1 & & 12.888 & 255347.734 & 6817632.500 & 98.4567 \\
\hline 2 & & 16.035 & 3489.019 & 106868.484 & 1.5433 \\
\hline Total & & & 258836.753 & 6924500.984 & 100.0000 \\
\hline
\end{tabular}

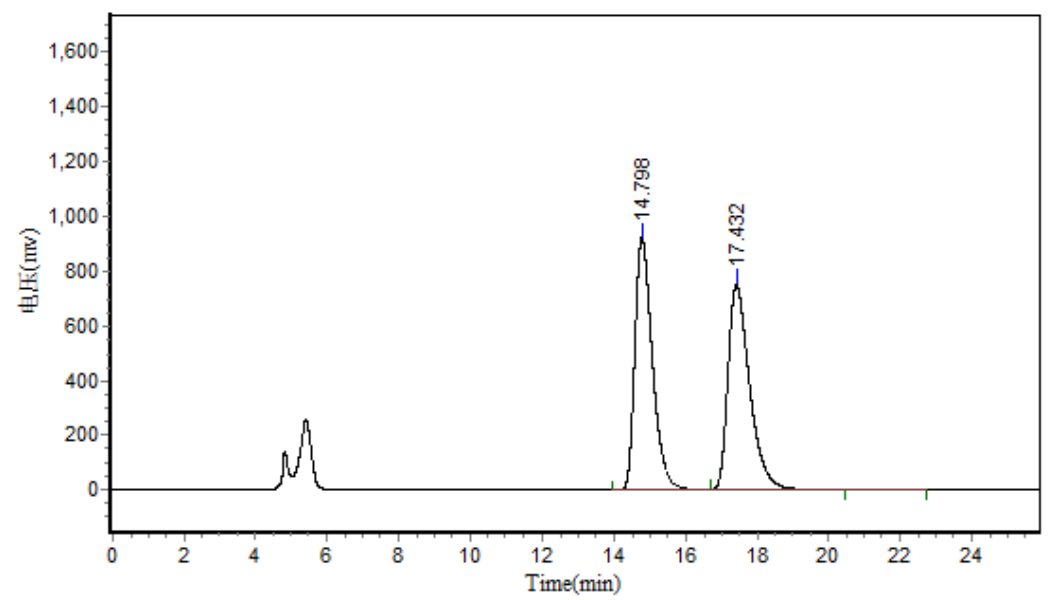

Results

\begin{tabular}{|c|c|c|c|c|c|}
\hline Peak No. & Peak ID & Ret Time & Height & Area & Conc. \\
\hline 1 & & 14.798 & 918173.375 & 31803374.000 & 50.3561 \\
\hline 2 & & 17.432 & 744133.063 & 31353524.000 & 49.6439 \\
\hline Total & & & 1662306.438 & 63156898.000 & 100.0000 \\
\hline
\end{tabular}


(3q)<smiles>CN1C(=O)[C@@](c2ccc(F)cc2)(c2ccc(Cl)cc2)NS1(=O)=O</smiles>

White solid, $95 \%$ yield, $96 \%$ ee; $\mathrm{mp} 170-172{ }^{\circ} \mathrm{C}$.

$[\alpha]_{\mathrm{D}}{ }^{25}=-10.9\left(c 0.49, \mathrm{CHCl}_{3}\right) ;{ }^{1} \mathrm{H}$ NMR $\left(300 \mathrm{MHz}, \mathrm{CDCl}_{3}\right) \delta 7.48-7.32$ $(\mathrm{m}, 6 \mathrm{H}), 7.08(\mathrm{t}, J=8.6 \mathrm{~Hz}, 2 \mathrm{H}), 5.12(\mathrm{~s}, 1 \mathrm{H}), 3.20(\mathrm{~s}, 3 \mathrm{H}) ;{ }^{13} \mathrm{C} \mathrm{NMR}$ $\left(125 \mathrm{MHz}, \mathrm{CDCl}_{3}\right) \delta 168.3,163.3\left(\mathrm{~d},{ }^{1} J_{\mathrm{CF}}=250.0 \mathrm{~Hz}\right), 136.4,135.9$, $133.8,129.8\left(\mathrm{~d},{ }^{3} J_{\mathrm{CF}}=8.5 \mathrm{~Hz}\right), 129.3,129.1,116.3\left(\mathrm{~d},{ }^{2} J_{\mathrm{CF}}=21.3 \mathrm{~Hz}\right)$, 74.5, 26.5; ${ }^{19} \mathrm{~F}$ NMR (471 MHz, $\left.\mathrm{CDCl}_{3}\right) \delta-111.2(\mathrm{~s})$; IR (KBr) v 3451, $3198,1708,1607,1506,1413,1345,1240,1193,1096,1071,969 \mathrm{~cm}^{-1}$; HRMS (ESI) for $\mathrm{C}_{15} \mathrm{H}_{12} \mathrm{O}_{3} \mathrm{~N}_{2} \mathrm{ClFSNa}[\mathrm{M}+\mathrm{Na}]^{+}$calcd 377.0133, found 377.0141.

HPLC (N-Boc derivative): Chiralpak AD-3 column $(250 \mathrm{~mm})$; detected at $220 \mathrm{~nm}$; hexane $/ i$-propanol $=97 / 3$; flow $=0.7 \mathrm{~mL} / \mathrm{min}$; Retention time: $13.6 \mathrm{~min}, 17.2$ (major).

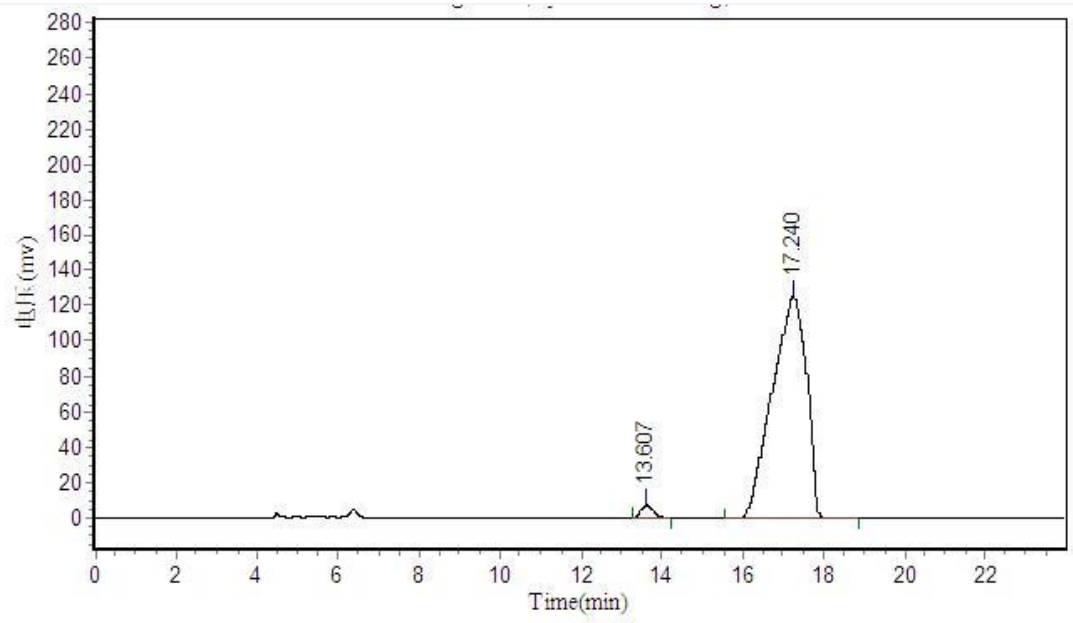

\begin{tabular}{|c|c|c|c|c|c|}
\hline \multicolumn{6}{|c|}{ Results } \\
\hline Peak No. & Peak ID & Ret Time & Height & Area & Conc. \\
\hline 1 & & 13.607 & 7516.544 & 164851.000 & 2.1883 \\
\hline 2 & & 17.240 & 125710.313 & 7368475.500 & 97.8117 \\
\hline Total & & & 133226.856 & 7533326.500 & 100.0000 \\
\hline
\end{tabular}

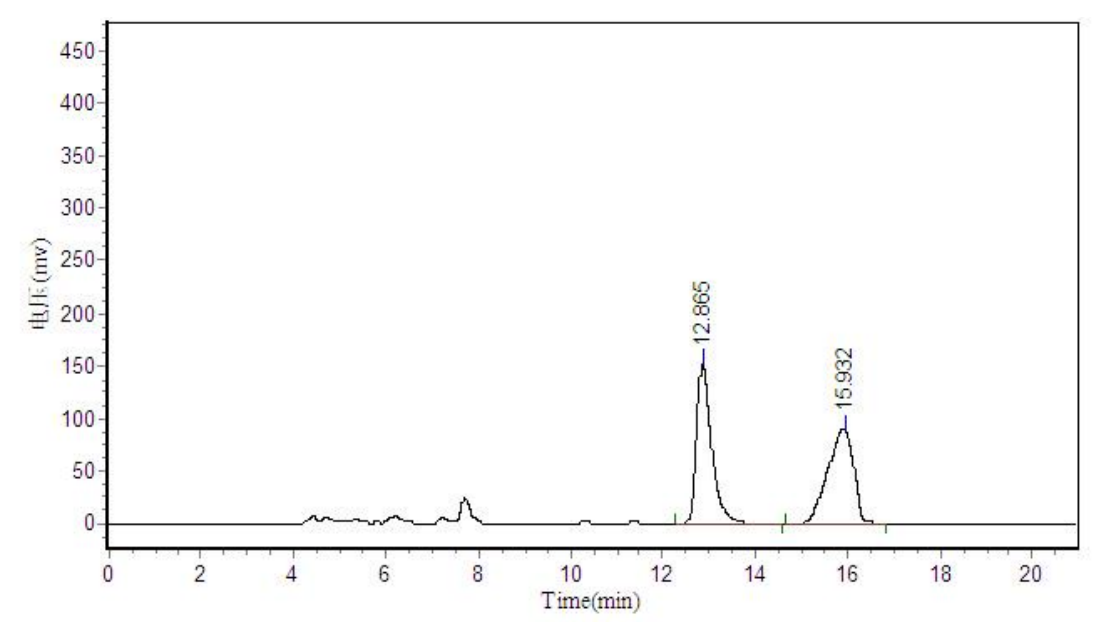

\begin{tabular}{|c|c|c|c|c|c|}
\hline \multicolumn{6}{|c|}{ Results } \\
\hline Peak No. & Peak ID & Ret Time & Height & Area & Conc. \\
\hline 1 & & 12.865 & 150064.844 & 3408933.000 & 49.8757 \\
\hline 2 & & 15.932 & 89131.930 & 3425918.000 & 50.1243 \\
\hline Total & & & 239196.773 & 6834851.000 & 100.0000 \\
\hline
\end{tabular}


(S)-4-(4-fluorophenyl)-2-methyl-4-(p-tolyl)-1,2,5-thiadiazolidin-3-one 1,1-dioxide (3p')<smiles></smiles>

White solid, $95 \%$ yield, $96 \%$ ee; $\mathrm{mp} 129-131{ }^{\circ} \mathrm{C}$.

$[\alpha]_{\mathrm{D}}{ }^{25}=+40.6\left(c 0.55, \mathrm{CHCl}_{3}\right) ;{ }^{1} \mathrm{H}$ NMR $\left(300 \mathrm{MHz}, \mathrm{CDCl}_{3}\right) \delta 7.58-7.46(\mathrm{~m}$, 2H), $7.24(\mathrm{~d}, J=8.2 \mathrm{~Hz}, 2 \mathrm{H}), 7.18(\mathrm{~d}, J=8.4 \mathrm{~Hz}, 2 \mathrm{H}), 7.12-6.99(\mathrm{~m}, 2 \mathrm{H})$, $5.05(\mathrm{~s}, 1 \mathrm{H}), 3.19(\mathrm{q}, J=1.1 \mathrm{~Hz}, 3 \mathrm{H}), 2.35(\mathrm{~s}, 3 \mathrm{H}) ;{ }^{13} \mathrm{C} \mathrm{NMR}(125 \mathrm{MHz}$, $\left.\mathrm{CDCl}_{3}\right) \delta 168.8,163.2\left(\mathrm{~d},{ }^{1} J_{\mathrm{CF}}=247.5 \mathrm{~Hz}\right), 139.8,135.7,133.7,130.0,129.8$ $\left(\mathrm{d},{ }^{3} J_{\mathrm{CF}}=8.8 \mathrm{~Hz}\right), 127.5,115.9\left(\mathrm{~d},{ }^{2} J_{\mathrm{CF}}=21.3 \mathrm{~Hz}\right), 115.8,75.1,26.4,21.3$; ${ }^{19} \mathrm{~F}$ NMR (471 MHz, $\mathrm{CDCl}_{3}$ ) $\delta-112.3$ (s); IR (KBr) v 3429, 3183, 1710, 1604, 1507, 1413, 1341, 1226, 1185, 1160, 1071, $969 \mathrm{~cm}^{-1}$; HRMS (ESI) for $\mathrm{C}_{16} \mathrm{H}_{16} \mathrm{O}_{3} \mathrm{~N}_{2} \mathrm{FS}[\mathrm{M}+\mathrm{H}]^{+}$calcd 335.0860, found 335.0858 .

HPLC ( $N$-Boc derivative): Chiralpak IC column $(250 \mathrm{~mm})$; detected at $220 \mathrm{~nm}$; hexane $/ i$-propanol $=97 / 3$; flow $=0.7 \mathrm{~mL} / \mathrm{min}$; Retention time: $13.6 \mathrm{~min}$ (major), $15.6 \mathrm{~min}$

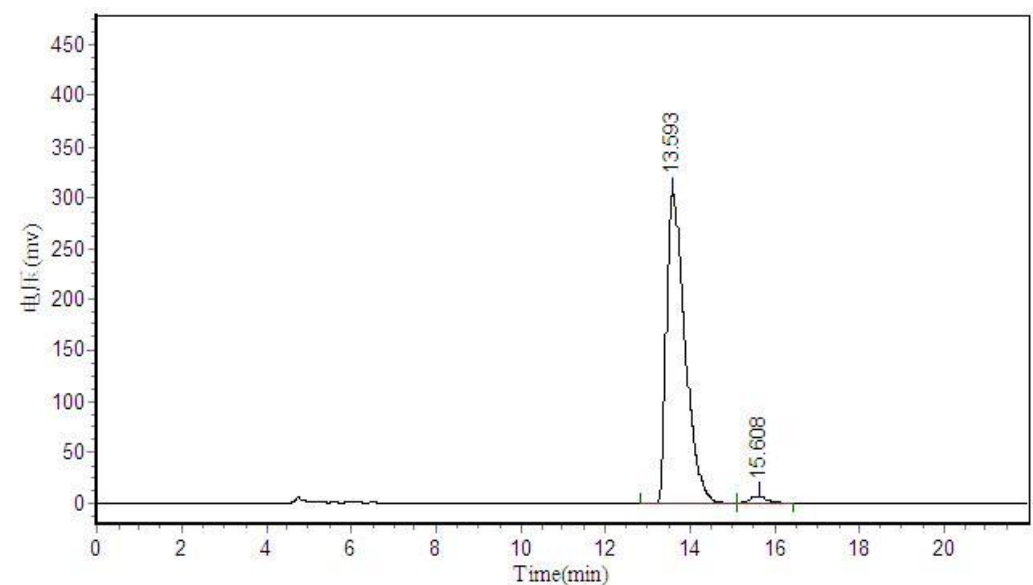

Results

\begin{tabular}{cccccc} 
Peak No. & Peak ID & Ret Time & Height & Area & Conc. \\
\hline 1 & 13.593 & 304857.063 & 9263543.000 & 97.8800 \\
2 & 15.608 & 6143.436 & 200642.578 & 2.1200 & 100.0000
\end{tabular}

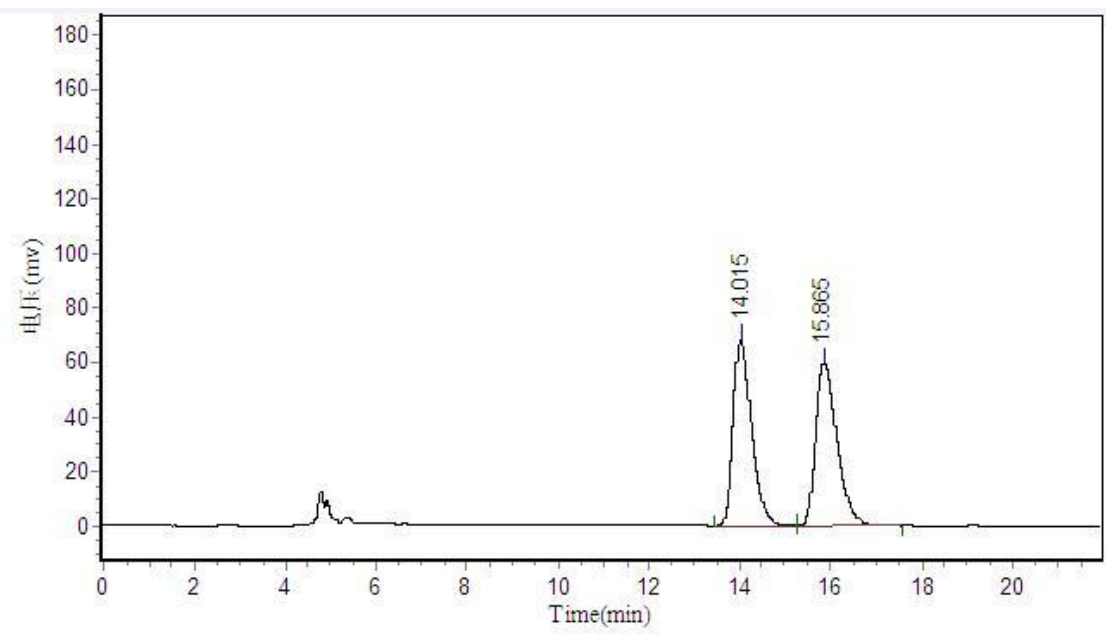

Results

\begin{tabular}{|c|c|c|c|c|c|}
\hline Peak No. & Peak ID & Ret Time & Height & Area & Conc. \\
\hline 1 & & 14.015 & 67825.938 & 1929191.500 & 49.7894 \\
\hline 2 & & 15.865 & 59565.824 & 1945513.500 & 50.2106 \\
\hline Total & & & 127391.762 & 3874705.000 & 100.0000 \\
\hline
\end{tabular}


(R)-4-ethoxy-3-phenyl-3-(p-tolyl)-2,3-dihydro-1,2,5-thiadiazole 1,1-dioxide (5a)

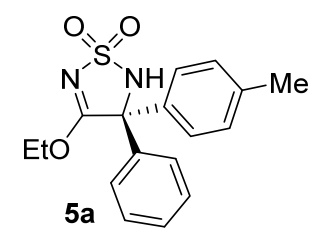

White solid, $95 \%$ yield, $99 \%$ ee; $\mathrm{mp} 134-136{ }^{\circ} \mathrm{C}$.

$[\alpha]_{\mathrm{D}}{ }^{20}=+8.8\left(\mathrm{c} 1.01, \mathrm{CHCl}_{3}\right)$; The absolute configuration was determined to be $(R)$ according to literature $\left[\mathrm{lit}^{\mathrm{ref}}[\alpha]_{\mathrm{D}}{ }^{22}=+8\left(c 1.0 \mathrm{CHCl}_{3}\right)\right]$.

${ }^{1} \mathrm{H}$ NMR $\left(300 \mathrm{MHz}, \mathrm{CDCl}_{3}\right) \delta 7.40(\mathrm{dt}, J=4.6,1.9 \mathrm{~Hz}, 5 \mathrm{H}), 7.25(\mathrm{~d}, J=$ $5.9 \mathrm{~Hz}, 2 \mathrm{H}), 7.18(\mathrm{~d}, J=8.1 \mathrm{~Hz}, 2 \mathrm{H}), 4.87(\mathrm{~s}, 1 \mathrm{H}), 4.51(\mathrm{q}, J=7.1 \mathrm{~Hz}, 2 \mathrm{H})$, 2.37 (s, 3H), 1.37 (t, $J=7.1 \mathrm{~Hz}, 3 \mathrm{H}) ;{ }^{13} \mathrm{C}$ NMR $\left(100 \mathrm{MHz}, \mathrm{CDCl}_{3}\right) \delta 177.3,139.4,138.2,135.4$, 129.7, 129.3, 128.9, 127.9, 127.8, 70.1, 21.3, 13.9; HRMS (ESI) for $\mathrm{C}_{17} \mathrm{H}_{18} \mathrm{O}_{3} \mathrm{~N}_{2} \mathrm{NaS}[\mathrm{M}+\mathrm{Na}]^{+}$ calcd 353.0936, found 353.0927 .

HPLC: Chiralpak IC column $(250 \mathrm{~mm})$; detected at $220 \mathrm{~nm}$; hexane $/ i$-propanol $=70 / 30$; flow $=$ $0.7 \mathrm{~mL} / \mathrm{min}$; Retention time: $30.6 \mathrm{~min}, 34.9 \mathrm{~min}$ (major).

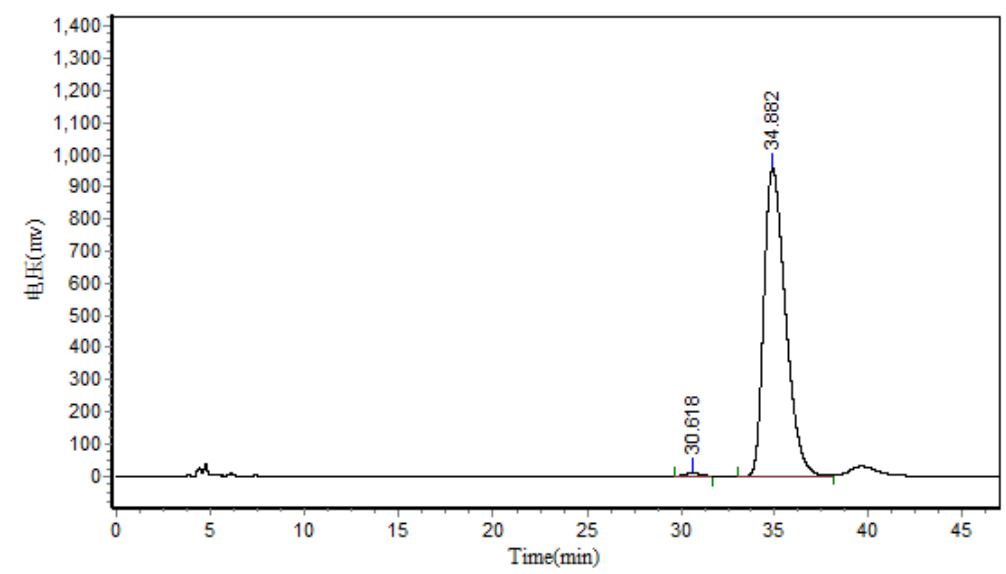

\begin{tabular}{|c|c|c|c|c|c|}
\hline \multicolumn{6}{|c|}{ Results } \\
\hline Peak No. & Peak ID & Ret Time & Height & Area & Conc. \\
\hline 1 & & 30.618 & 8925.209 & 522309.563 & 0.6749 \\
\hline 2 & & 34.882 & 959258.375 & 76867672.000 & 99.3251 \\
\hline Total & & & 968183.584 & 77389981.563 & 100.0000 \\
\hline
\end{tabular}

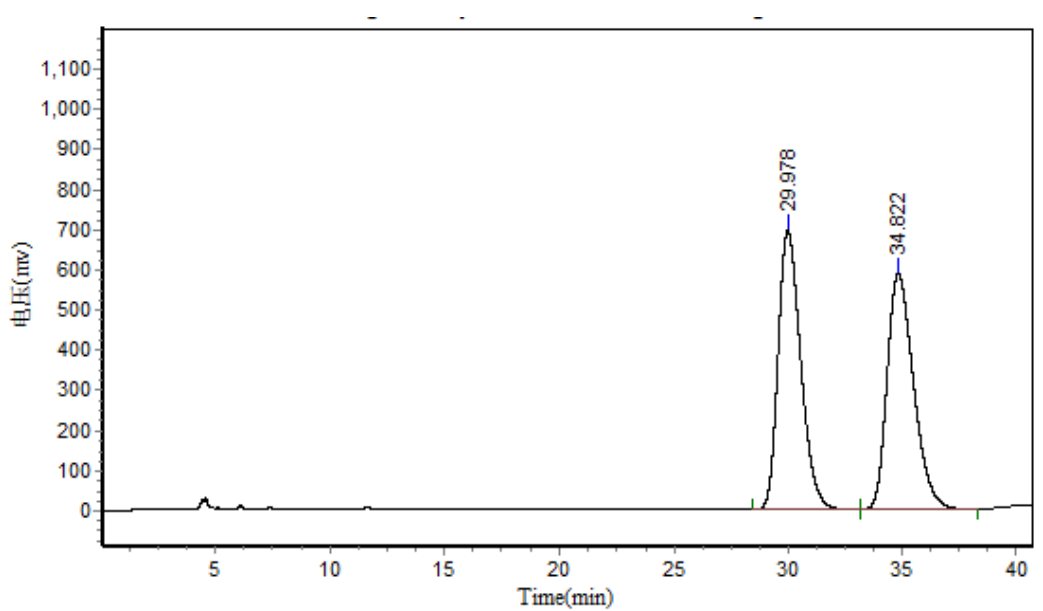

Results

\begin{tabular}{|c|c|c|c|c|c|}
\hline Peak No. & Peak ID & Ret Time & Height & Area & Conc. \\
\hline 1 & & 29.978 & 694174.188 & 47732172.000 & 50.2189 \\
\hline 2 & & 34.822 & 587173.750 & 47315968.000 & 49.7811 \\
\hline Total & & & 1281347.938 & 95048140.000 & 100.0000 \\
\hline
\end{tabular}

Ref: Nishimura, T.; Ebe, Y.; Fujimoto, H.; Hayashi, T. Chem. Commun. 2013, 49, 5504. 
(R)-3-(4-chlorophenyl)-4-ethoxy-3-phenyl-2,3-dihydro-1,2,5-thiadiazole 1,1-dioxide (5b)<smiles>CCOC1=NS(=O)(=O)NC1(c1ccccc1)c1ccc(Cl)cc1</smiles>

White solid, $95 \%$ yield, $98 \%$ ee; $\mathrm{mp} 129-131{ }^{\circ} \mathrm{C}$.

$[\alpha]_{\mathrm{D}}{ }^{25}=-69.8\left(c 0.455, \mathrm{CHCl}_{3}\right) ;{ }^{1} \mathrm{H}$ NMR $\left(300 \mathrm{MHz}, \mathrm{CDCl}_{3}\right) \delta 7.52-7.32$

$(\mathrm{m}, 7 \mathrm{H}), 7.32-7.24(\mathrm{~m}, 2 \mathrm{H}), 5.14(\mathrm{~s}, 1 \mathrm{H}), 4.51(\mathrm{qd}, J=7.0,3.3 \mathrm{~Hz}, 2 \mathrm{H})$,

$1.37(\mathrm{t}, J=7.1 \mathrm{~Hz}, 3 \mathrm{H}) .{ }^{13} \mathrm{C}$ NMR $\left(100 \mathrm{MHz}, \mathrm{CDCl}_{3}\right) \delta 176.6,138.3,136.2$,

135.5, 129.7, 129.5, 129.3, 129.1, 127.7, 70.4, 13.9; IR (KBr) v 3248, 1613,

1492, 1380, 1321, 1184, 1160, 1095, 1011, $955 \mathrm{~cm}^{-1}$; HRMS (ESI) for $\mathrm{C}_{16} \mathrm{H}_{15} \mathrm{O}_{3} \mathrm{~N}_{2} \mathrm{NaSCl}$ $[\mathrm{M}+\mathrm{Na}]^{+}$calcd 373.0390, found 373.0400 .

HPLC: Chiralpak IC column $(250 \mathrm{~mm})$; detected at $220 \mathrm{~nm}$; hexane $/ i$-propanol $=70 / 30$; flow $=$ $0.7 \mathrm{~mL} / \mathrm{min}$; Retention time: $21.6 \mathrm{~min}, 30.9 \mathrm{~min}$ (major).

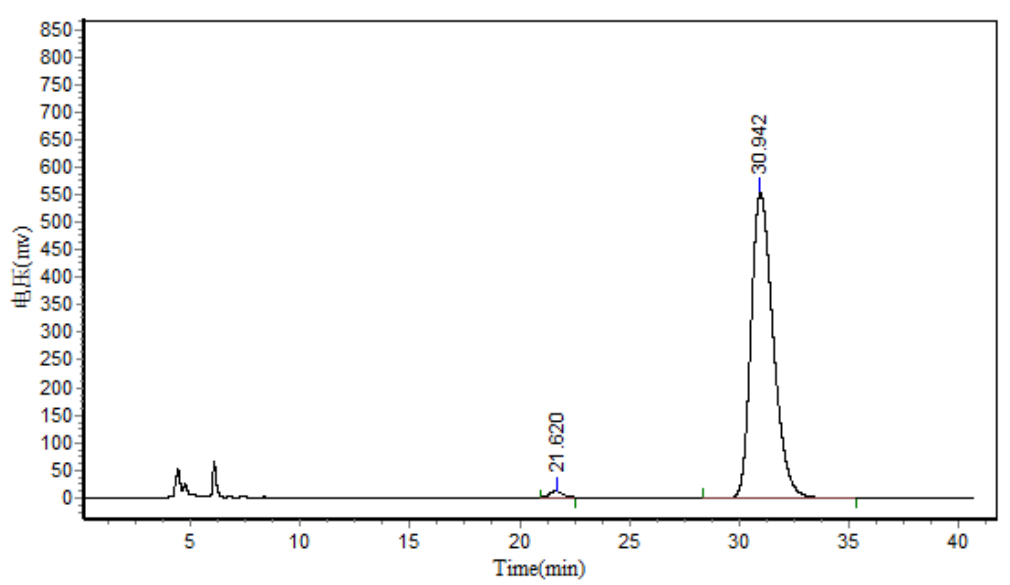

Results

\begin{tabular}{|c|c|c|c|c|c|}
\hline Peak No. & Peak ID & Ret Time & Height & Area & Conc. \\
\hline 1 & & 21.620 & 10878.356 & 481431.406 & 1.2283 \\
\hline 2 & & 30.942 & 554072.500 & 38713296.000 & 98.7717 \\
\hline Total & & & 564950.856 & 39194727.406 & 100.0000 \\
\hline
\end{tabular}

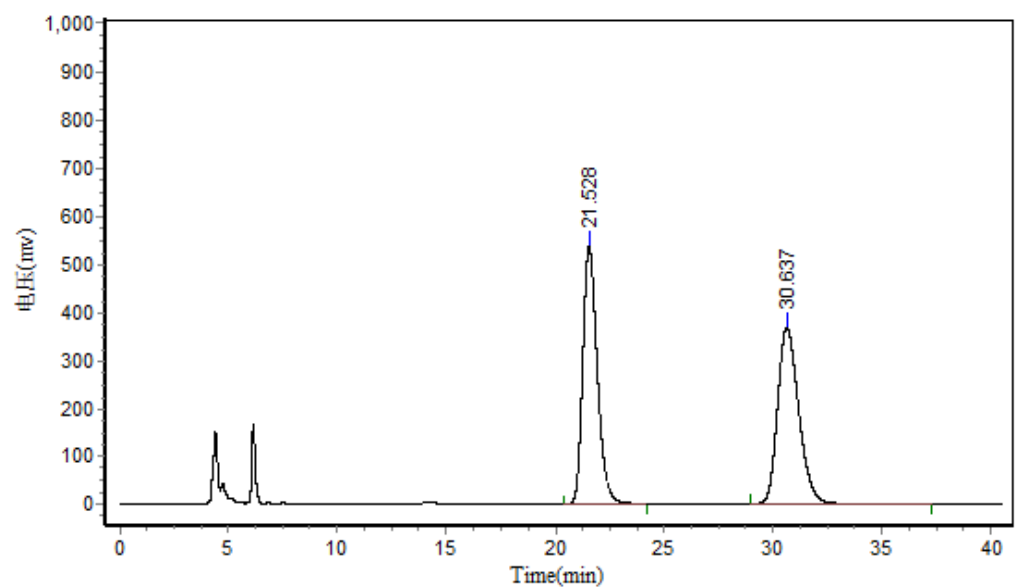

Results

\begin{tabular}{|c|c|c|c|c|c|}
\hline Peak No. & Peak ID & Ret Time & Height & Area & Conc. \\
\hline 1 & & 21.528 & 535973.250 & 40794.000 & 0.1640 \\
\hline 2 & & 30.637 & 366811.844 & 24827062.000 & 99.8360 \\
\hline Total & & & 902785.094 & 24867856.000 & 100.0000 \\
\hline
\end{tabular}


(R)-4-ethoxy-3-(4-methoxyphenyl)-3-phenyl-2,3-dihydro-1,2,5-thiadiazole 1,1-dioxide (5c)<smiles>CCOC1=NS(=O)(=O)NC1(c1ccccc1)c1ccc(OC)cc1</smiles>

Yellow oil, 97\% yield, 98\% ee;

$[\alpha]_{\mathrm{D}}{ }^{25}=-9.1\left(c 0.405, \mathrm{CHCl}_{3}\right) ;{ }^{1} \mathrm{H} \mathrm{NMR}\left(300 \mathrm{MHz}, \mathrm{CDCl}_{3}\right) \delta 7.39(\mathrm{t}, J=$ $3.4 \mathrm{~Hz}, 5 \mathrm{H}), 7.29(\mathrm{~d}, J=8.8 \mathrm{~Hz}, 2 \mathrm{H}), 6.88(\mathrm{~d}, J=8.8 \mathrm{~Hz}, 2 \mathrm{H}), 5.03(\mathrm{~s}$, $1 \mathrm{H}), 4.50(\mathrm{q}, J=7.1 \mathrm{~Hz}, 2 \mathrm{H}), 3.81(\mathrm{~s}, 3 \mathrm{H}), 1.36(\mathrm{t}, J=7.1 \mathrm{~Hz}, 3 \mathrm{H}) ;{ }^{13} \mathrm{C}$ NMR $\left(150 \mathrm{MHz}, \mathrm{CDCl}_{3}\right) \delta 177.4,160.3,138.4,130.2,129.30,129.25$, 129.0, 127.9, 114.3, 70.1, 55.5, 13.9; IR (KBr) v 3432, 1618, 1511, 1383, 1325, $1167 \mathrm{~cm}^{-1}$; HRMS (ESI) for $\mathrm{C}_{17} \mathrm{H}_{18} \mathrm{O}_{4} \mathrm{~N}_{2} \mathrm{NaS}[\mathrm{M}+\mathrm{Na}]^{+}$calcd 369.0885, found 369.0888 .

HPLC: Chiralpak IC column $(250 \mathrm{~mm})$; detected at $220 \mathrm{~nm}$; hexane $/ i$-propanol $=70 / 30$; flow $=$ $0.7 \mathrm{~mL} / \mathrm{min}$; Retention time: $37.5 \mathrm{~min}, 54.4 \mathrm{~min}$ (major).

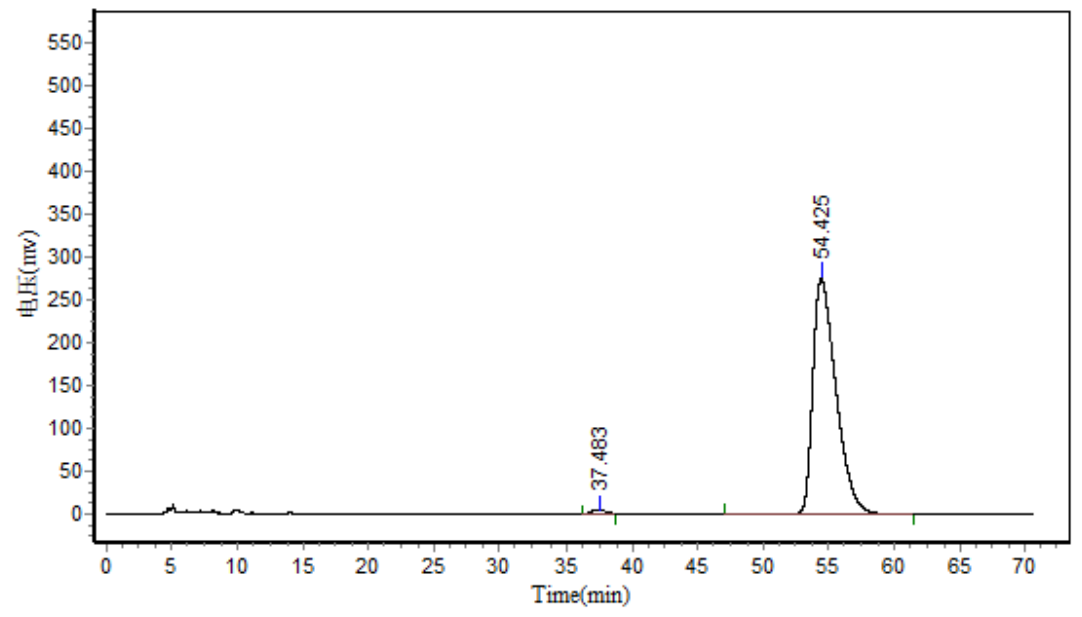

Results

\begin{tabular}{|c|c|c|c|c|c|}
\hline Peak No. & Peak ID & Ret Time & Height & Area & Conc. \\
\hline 1 & & 37.483 & 5226.967 & 380999.219 & 1.1207 \\
\hline 2 & & 54.425 & 274467.406 & 33614368.000 & 98.8793 \\
\hline Total & & & 279694.374 & 33995367.219 & 100.0000 \\
\hline
\end{tabular}

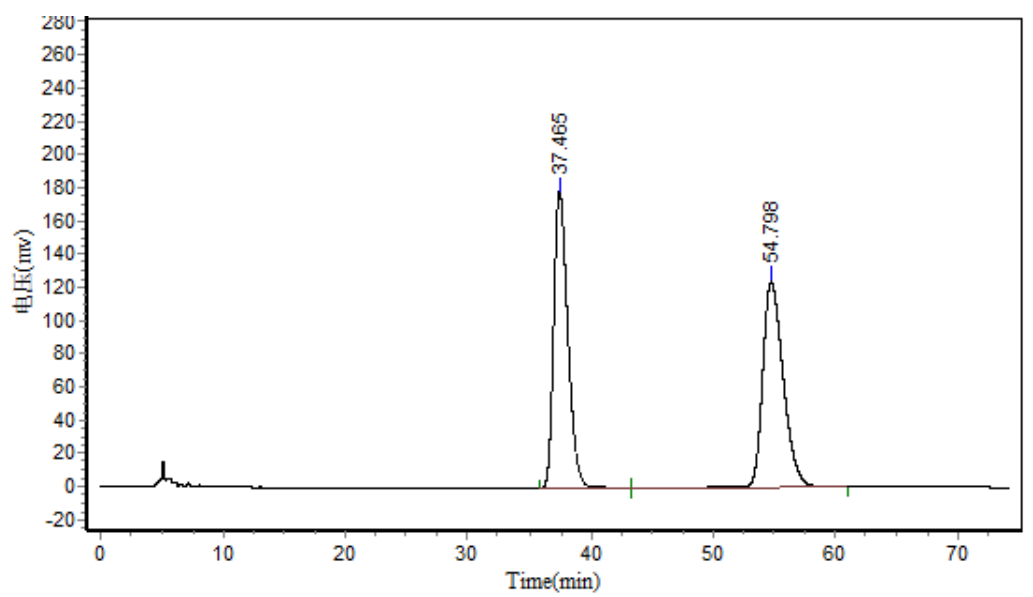

Results

\begin{tabular}{|c|c|c|c|c|c|}
\hline Peak No. & Peak ID & Ret Time & Height & Area & Conc. \\
\hline 1 & & 37.465 & 179123.594 & 14416071.000 & 49.2456 \\
\hline 2 & & 54.798 & 124247.141 & 14857746.000 & 50.7544 \\
\hline Total & & & 303370.734 & 29273817.000 & 100.0000 \\
\hline
\end{tabular}


(R)-4-ethoxy-3-phenyl-3-(m-tolyl)-2,3-dihydro-1,2,5-thiadiazole 1,1-dioxide (5d)

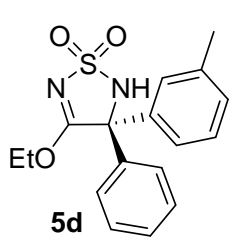

White solid, $93 \%$ yield, $94 \%$ ee; $\mathrm{mp} 83-85^{\circ} \mathrm{C}$.

$[\alpha]_{\mathrm{D}}{ }^{25}=+18.2\left(c 0.425, \mathrm{CHCl}_{3}\right) ;{ }^{1} \mathrm{H} \mathrm{NMR}\left(300 \mathrm{MHz}, \mathrm{CDCl}_{3}\right) \delta 7.40(\mathrm{dd}, J=$ 10.7, $5.0 \mathrm{~Hz}, 5 \mathrm{H}), 7.26(\mathrm{dd}, J=9.0,5.9 \mathrm{~Hz}, 1 \mathrm{H}), 7.16(\mathrm{dd}, J=16.0,9.2 \mathrm{~Hz}$, $3 \mathrm{H}), 4.88(\mathrm{~s}, 1 \mathrm{H}), 4.51(\mathrm{q}, J=7.1 \mathrm{~Hz}, 2 \mathrm{H}), 2.34(\mathrm{~s}, 3 \mathrm{H}), 1.37(\mathrm{t}, J=7.1 \mathrm{~Hz}$, $3 \mathrm{H}) ;{ }^{13} \mathrm{C}$ NMR $\left(125 \mathrm{MHz}, \mathrm{CDCl}_{3}\right) \delta 177.2,139.0,138.3,138.1,130.1,129.3$, 128.9, 128.3, 127.9, 125.0, 70.1, 21.7, 13.9; IR (KBr) v 3448, 3313, 1612, 1365, 1320, 1171, 1158 , 1061, $1010 \mathrm{~cm}^{-1}$; HRMS (ESI) for $\mathrm{C}_{17} \mathrm{H}_{18} \mathrm{O}_{3} \mathrm{~N}_{2} \mathrm{NaS}[\mathrm{M}+\mathrm{Na}]^{+}$calcd 353.0936, found 353.0927 .

HPLC: Chiralpak AD-H column $(250 \mathrm{~mm})$; detected at $220 \mathrm{~nm}$; hexane $/ i$-propanol $=80 / 20$; flow $=0.7 \mathrm{~mL} / \mathrm{min}$; Retention time: $11.1 \mathrm{~min}$ (major), $12.9 \mathrm{~min}$.

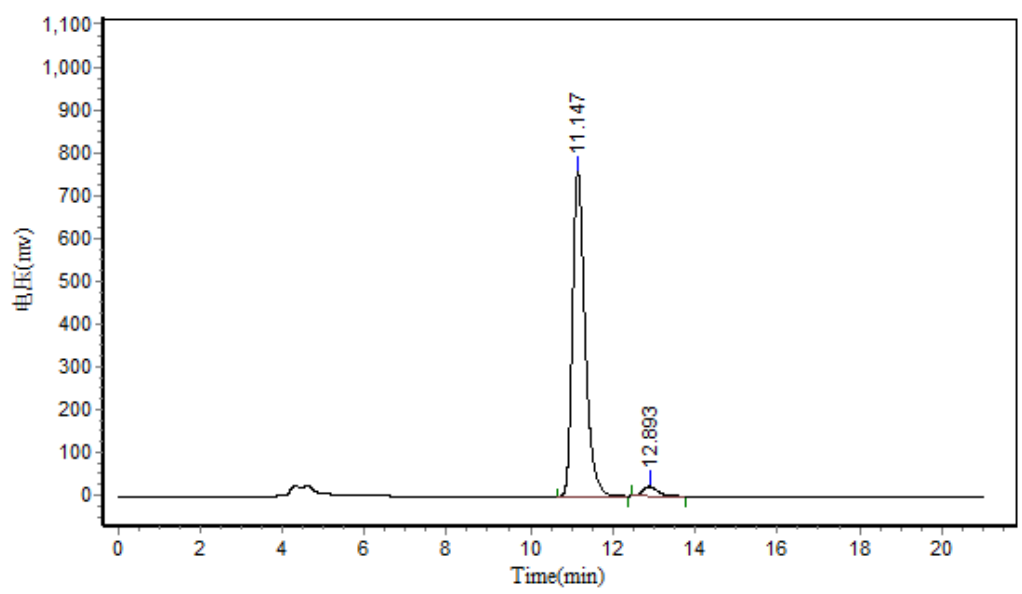

\begin{tabular}{|c|c|c|c|c|c|}
\hline \multicolumn{6}{|c|}{ Results } \\
\hline Peak No. & Peak ID & Ret Time & Height & Area & Conc. \\
\hline 1 & & 11.147 & 757685.438 & 16163664.000 & 96.7276 \\
\hline 2 & & 12.893 & 21930.197 & 546842.313 & 3.2724 \\
\hline Total & & & 779615.635 & 16710506.313 & 100.0000 \\
\hline
\end{tabular}

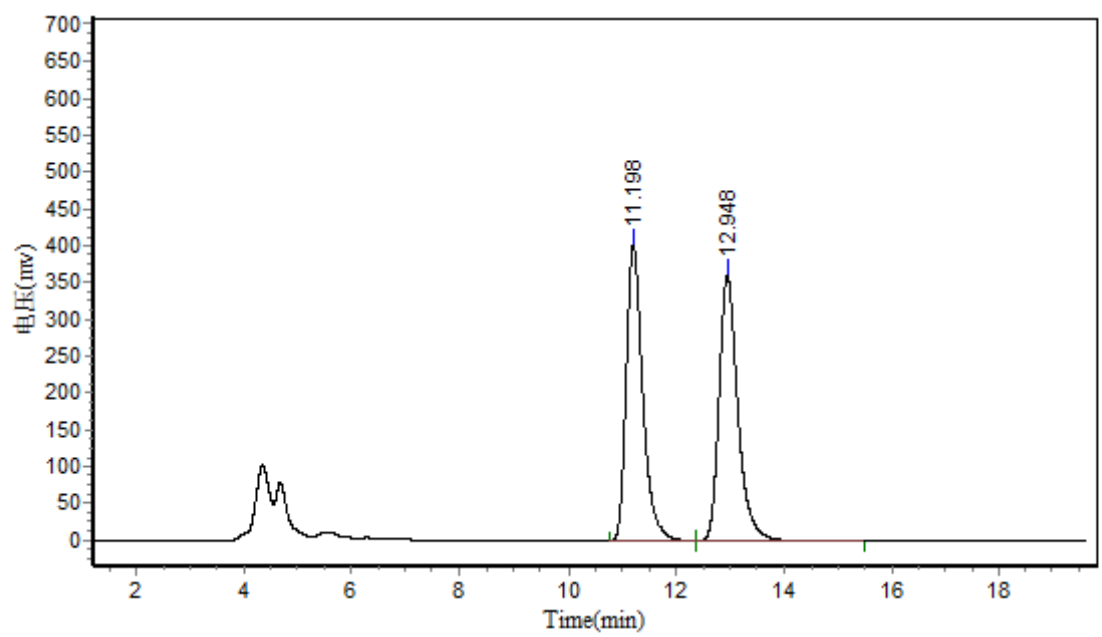

Results

\begin{tabular}{|c|c|c|c|c|c|}
\hline Peak No. & Peak ID & Ret Time & Height & Area & Conc. \\
\hline 1 & & 11.198 & 400726.031 & 8575244.000 & 49.6663 \\
\hline 2 & & 12.948 & 359100.563 & 8690474.000 & 50.3337 \\
\hline Total & & & 759826.594 & 17265718.000 & 100.0000 \\
\hline
\end{tabular}


(R)-4-ethoxy-3-(3-fluorophenyl)-3-phenyl-2,3-dihydro-1,2,5-thiadiazole 1,1-dioxide (5e)

White solid, $92 \%$ yield, $97 \%$ ee; $\mathrm{mp} 108-111^{\circ} \mathrm{C}$.

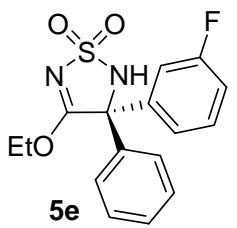

$[\alpha]_{\mathrm{D}}{ }^{25}=-48.1\left(c \quad 0.52, \mathrm{CHCl}_{3}\right) ;{ }^{1} \mathrm{H}$ NMR $\left(300 \mathrm{MHz}, \mathrm{CDCl}_{3}\right) \delta 7.47-7.35(\mathrm{~m}$, $4 \mathrm{H}), 7.35-7.26(\mathrm{~m}, 3 \mathrm{H}), 7.18(\mathrm{~d}, J=10.0 \mathrm{~Hz}, 1 \mathrm{H}), 7.10(\mathrm{t}, J=8.1 \mathrm{~Hz}, 1 \mathrm{H})$, $4.95(\mathrm{~s}, 1 \mathrm{H}), 4.53(\mathrm{qd}, J=7.1,2.1 \mathrm{~Hz}, 2 \mathrm{H}), 1.39(\mathrm{t}, J=7.1 \mathrm{~Hz}, 3 \mathrm{H}) ;{ }^{13} \mathrm{C} \mathrm{NMR}$ $\left(150 \mathrm{MHz}, \mathrm{CDCl}_{3}\right) \delta 176.5,162.8\left(\mathrm{~d},{ }^{1} J_{\mathrm{CF}}=246.0 \mathrm{~Hz}\right), 140.2\left(\mathrm{~d},{ }^{3} J_{\mathrm{CF}}=7.2 \mathrm{~Hz}\right)$, $138.2,130.5\left(\mathrm{~d},{ }^{3} \mathrm{~J}=8.2 \mathrm{~Hz}\right), 129.7,129.3,127.7,123.7\left(\mathrm{~d},{ }^{4} J=2.9 \mathrm{~Hz}\right), 116.4$ $\left(\mathrm{d},{ }^{2} J_{\mathrm{CF}}=21.0 \mathrm{~Hz}\right), 115.5\left(\mathrm{~d},{ }^{2} J_{\mathrm{CF}}=24.0 \mathrm{~Hz}\right), 70.4,13.9 ;{ }^{19} \mathrm{~F} \mathrm{NMR}\left(471 \mathrm{MHz}, \mathrm{CDCl}_{3}\right) \delta-111.1$ (dd, $J=9.4,14.1 \mathrm{~Hz}$ ); IR (KBr) v 3448, 3269, 1618, 1593, 1379, 1317, 1181, 1137, $946 \mathrm{~cm}^{-1}$; HRMS (ESI) for $\mathrm{C}_{16} \mathrm{H}_{15} \mathrm{O}_{3} \mathrm{~N}_{2} \mathrm{NaSF}[\mathrm{M}+\mathrm{Na}]^{+}$calcd 357.0685, found 357.0687 .

HPLC: Chiralpak IC column $(250 \mathrm{~mm})$; detected at $220 \mathrm{~nm}$; hexane $/ i$-propanol $=70 / 30$; flow $=$ $0.7 \mathrm{~mL} / \mathrm{min}$; Retention time: $19.9 \mathrm{~min}, 24.9 \mathrm{~min}$ (major).

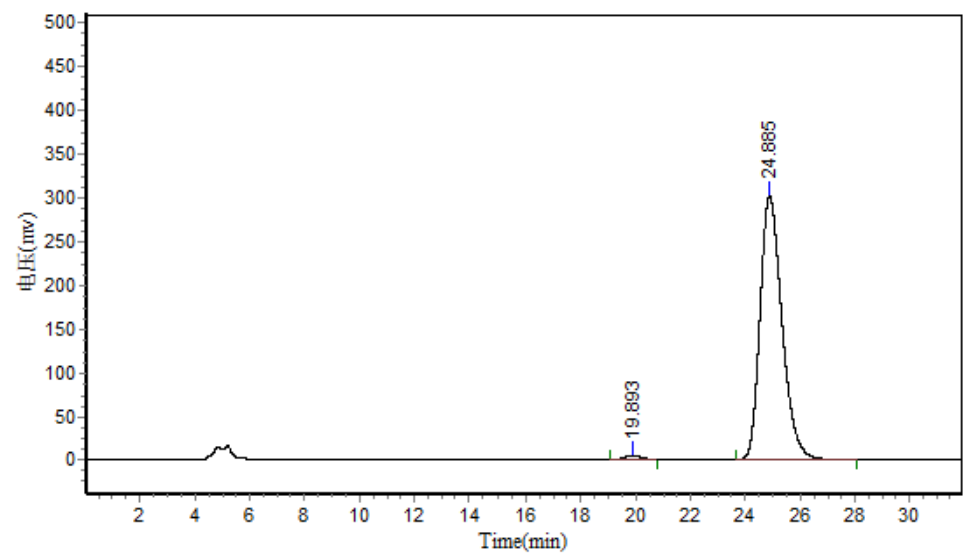

Results

\begin{tabular}{|c|c|c|c|c|c|}
\hline \multicolumn{6}{|c|}{ 10 } \\
\hline Peak No. & Peak ID & Ret Time & Height & Area & Conc. \\
\hline 1 & & 19.893 & 5276.383 & 220847.547 & 1.3037 \\
\hline 2 & & 24.885 & 301204.781 & 16718641.000 & 98.6963 \\
\hline Total & & & 306481.164 & 16939488.547 & 100.0000 \\
\hline
\end{tabular}

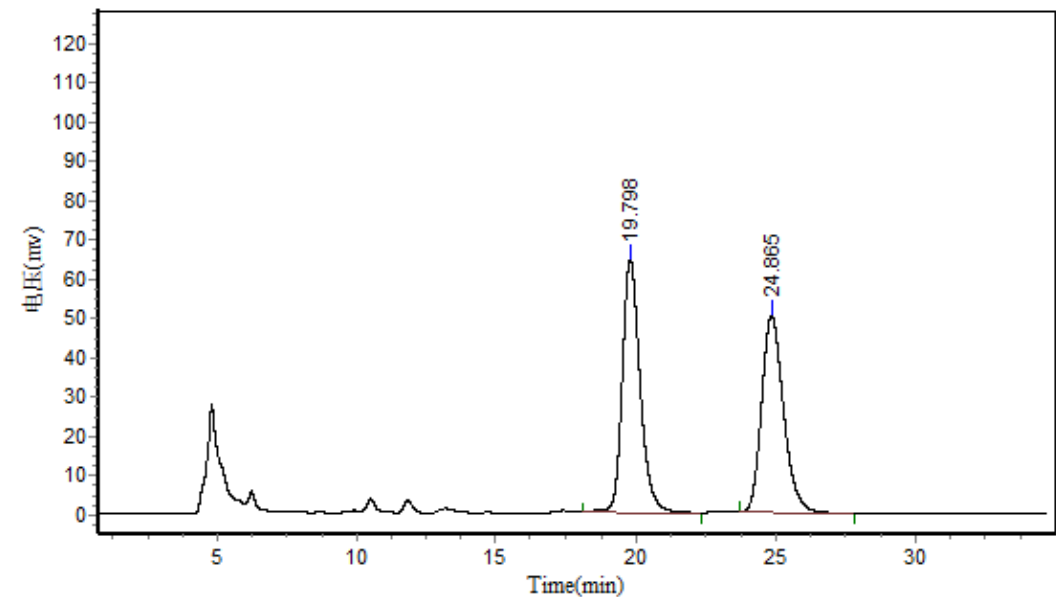

\begin{tabular}{|c|c|c|c|c|c|}
\hline \multicolumn{6}{|c|}{ Results } \\
\hline Peak No. & Peak ID & Ret Time & Height & Area & Conc. \\
\hline 1 & & 19.798 & 64605.797 & 2910375.000 & 51.1446 \\
\hline 2 & & 24.865 & 50216.047 & 2780109.750 & 48.8554 \\
\hline Total & & & 114821.844 & 5690484.750 & 100.0000 \\
\hline
\end{tabular}


(R)-4-ethoxy-3-(naphthalen-2-yl)-3-phenyl-2,3-dihydro-1,2,5-thiadiazole 1,1-dioxide (5f)<smiles>CCOC1(c2ccccc2)NS(=O)(=O)NC1c1ccc2ccccc2c1</smiles>
Yellow solid, $72 \%$ yield, $96 \%$ ee; $\mathrm{mp} 113-115^{\circ} \mathrm{C}$. $[\alpha]_{\mathrm{D}}{ }^{25}=-29.1\left(\right.$ c $\left.0.44, \mathrm{CHCl}_{3}\right) ;{ }^{1} \mathrm{H}$ NMR $\left(300 \mathrm{MHz}, \mathrm{CDCl}_{3}\right) \delta 8.00(\mathrm{~s}, 1 \mathrm{H})$, $7.84(\mathrm{~d}, J=8.4 \mathrm{~Hz}, 3 \mathrm{H}), 7.54(\mathrm{dd}, J=9.1,5.3 \mathrm{~Hz}, 2 \mathrm{H}), 7.45-7.32(\mathrm{~m}, 6 \mathrm{H})$, $5.01(\mathrm{~s}, 1 \mathrm{H}), 4.54(\mathrm{q}, J=7.1 \mathrm{~Hz}, 2 \mathrm{H}), 1.38(\mathrm{t}, J=7.1 \mathrm{~Hz}, 3 \mathrm{H}) ;{ }^{13} \mathrm{C} \mathrm{NMR}$ $\left(150 \mathrm{MHz}, \mathrm{CDCl}_{3}\right) \delta 177.1,138.3,135.2,133.4,132.9,129.5,129.1,128.9$, 128.8, 127.9, 127.7, 127.6, 127.4, 127.0, 125.1, 70.3, 13.9; IR (KBr) v 3440, 1612, 1381, 1322, 1177, 1162, 1010, 941, $862 \mathrm{~cm}^{-1}$; HRMS (ESI) for $\mathrm{C}_{20} \mathrm{H}_{18} \mathrm{O}_{3} \mathrm{~N}_{2} \mathrm{NaS}[\mathrm{M}+\mathrm{Na}]^{+}$calcd 389.0936, found 389.0938 .

HPLC: Chiralpak IC column $(250 \mathrm{~mm})$; detected at $220 \mathrm{~nm}$; hexane $/ i$-propanol $=70 / 30$; flow $=$ $0.7 \mathrm{~mL} / \mathrm{min}$; Retention time: $30.8 \mathrm{~min}, 69.2 \mathrm{~min}$ (major).

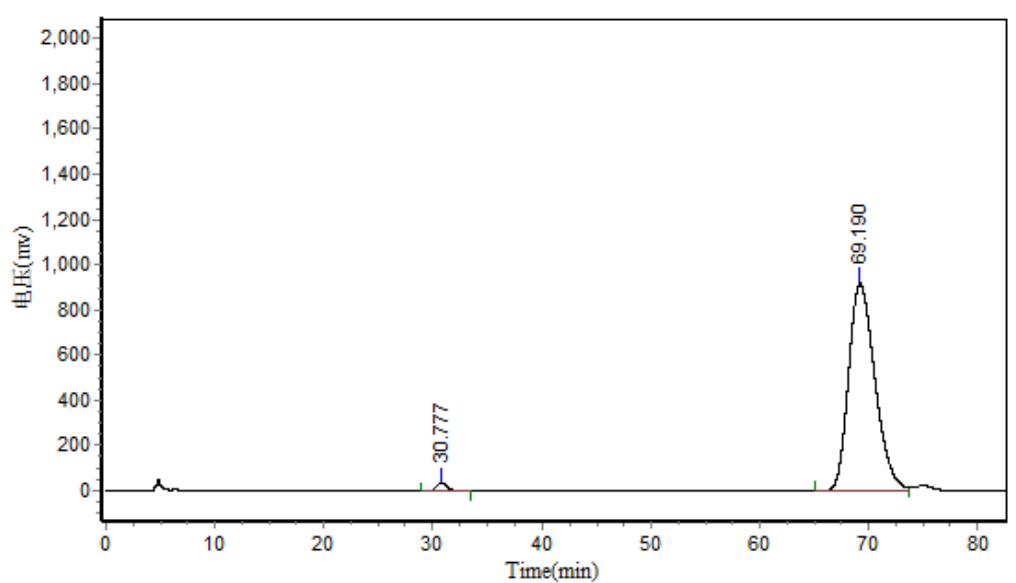

Results

\begin{tabular}{|c|c|c|c|c|c|}
\hline Peak No. & Peak ID & Ret Time & Height & Area & Conc. \\
\hline 1 & & 30.777 & 40064.422 & 3066839.750 & 1.9278 \\
\hline 2 & & 69.190 & 920792.188 & 156018272.000 & 98.0722 \\
\hline Total & & & 960856.609 & 159085111.750 & 100.000 \\
\hline
\end{tabular}

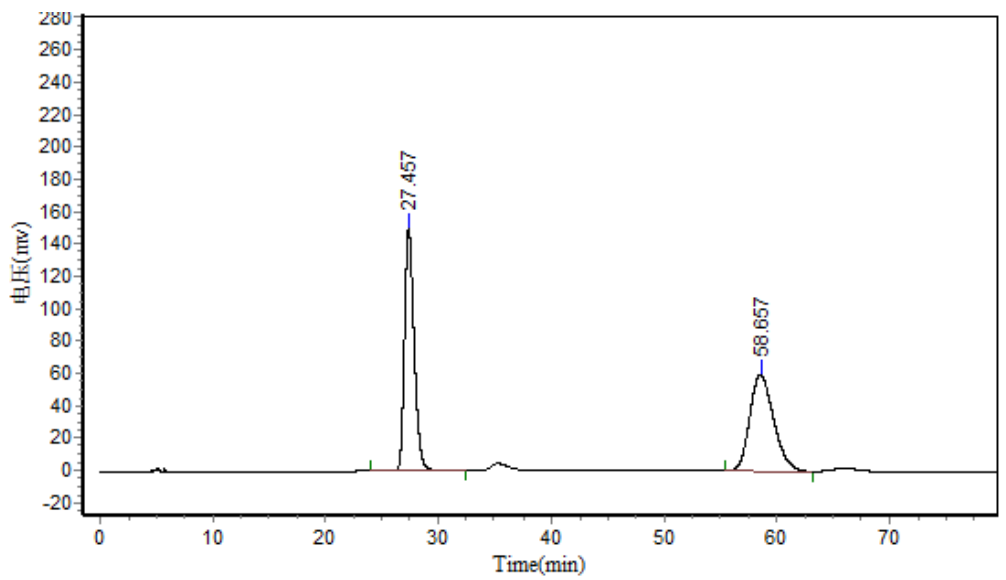

Results

\begin{tabular}{|c|c|c|c|c|c|}
\hline Peak No. & Peak ID & Ret Time & Height & Area & Conc. \\
\hline 1 & & 27.457 & 149366.000 & 8967123.000 & 50.2512 \\
\hline 2 & & 58.657 & 59754.063 & 8877466.000 & 49.7488 \\
\hline
\end{tabular}




\section{(R)-4-ethoxy-3-phenyl-3-(thiophen-3-yl)-2,3-dihydro-1,2,5-thiadiazole 1,1-dioxide (5g)}

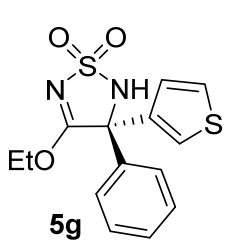

Yellow solid, $87 \%$ yield, $98 \%$ ee; $\mathrm{mp} 108-111^{\circ} \mathrm{C}$.

$[\alpha]_{\mathrm{D}}{ }^{25}=-16.6\left(c 0.435, \mathrm{CHCl}_{3}\right) ;{ }^{1} \mathrm{H}$ NMR $\left(300 \mathrm{MHz}, \mathrm{CDCl}_{3}\right) \delta 7.50-7.44(\mathrm{~m}$, $1 \mathrm{H}), 7.44-7.30(\mathrm{~m}, 6 \mathrm{H}), 6.99(\mathrm{dd}, J=5.0,0.9 \mathrm{~Hz}, 1 \mathrm{H}), 5.01(\mathrm{~s}, 1 \mathrm{H}), 4.51(\mathrm{q}, J$ $=7.1 \mathrm{~Hz}, 2 \mathrm{H}), 1.38(\mathrm{t}, J=7.1 \mathrm{~Hz}, 3 \mathrm{H}) ;{ }^{13} \mathrm{C} \mathrm{NMR}\left(125 \mathrm{MHz}, \mathrm{CDCl}_{3}\right) \delta 176.7$, 138.5, 138.1, 129.6, 129.2, 127.4, 127.3, 126.8, 125.7, 74.9, 70.2, 13.9; IR (KBr) $v$ 3024, 1614, 1379, 1309, 1169, 1150, 1090, 1006, $953 \mathrm{~cm}^{-1}$; HRMS (ESI) for $\mathrm{C}_{14} \mathrm{H}_{14} \mathrm{O}_{3} \mathrm{~N}_{2} \mathrm{NaS}_{2}$ $[\mathrm{M}+\mathrm{Na}]^{+}$calcd 345.0344 , found 345.0338 .

HPLC: Chiralpak AD-H column $(250 \mathrm{~mm})$; detected at $220 \mathrm{~nm}$; hexane $/ i$-propanol $=85 / 15$; flow $=0.7 \mathrm{~mL} / \mathrm{min}$; Retention time: $19.0 \mathrm{~min}, 22.4 \mathrm{~min}$ (major).

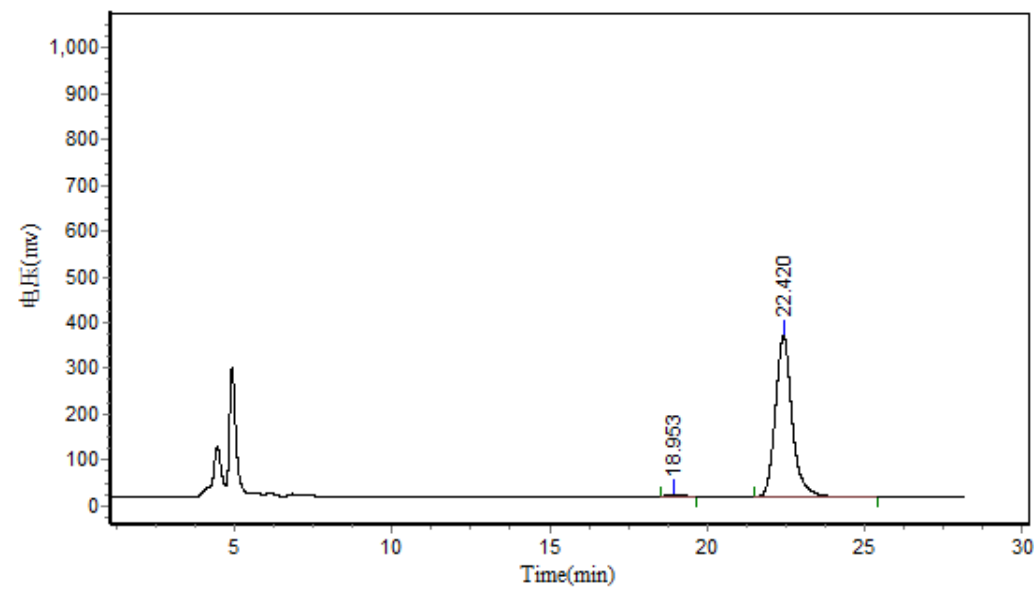

\begin{tabular}{|c|c|c|c|c|c|}
\hline \multicolumn{6}{|c|}{ Results } \\
\hline Peak No. & Peak ID & Ret Time & Height & Area & Conc. \\
\hline 1 & & 18.953 & 4670.670 & 140409.250 & 1.0679 \\
\hline 2 & & 22.420 & 350647.844 & 13007147.000 & 98.9321 \\
\hline Total & & & 355318.514 & 13147556.250 & 100.0000 \\
\hline
\end{tabular}

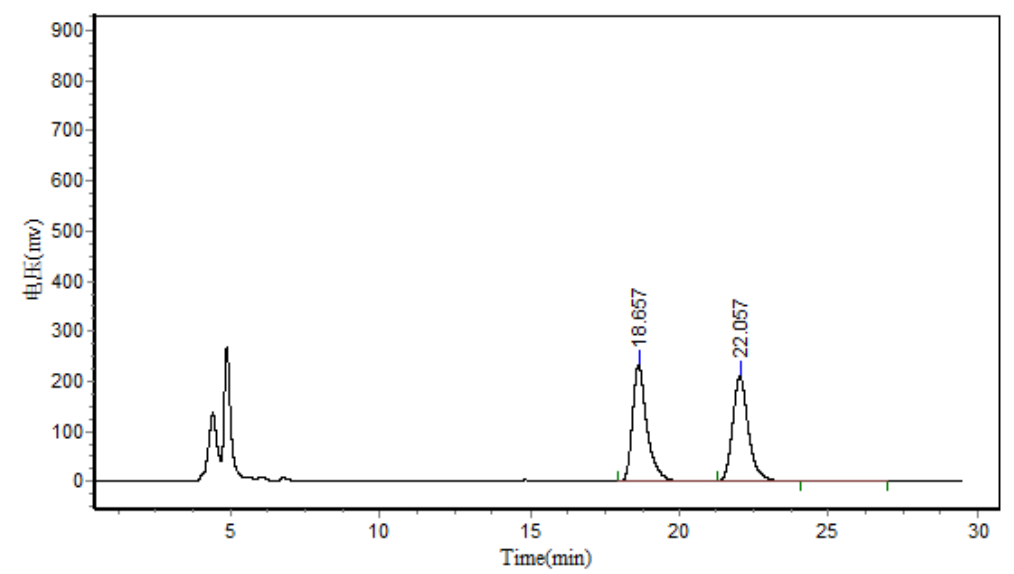

Results

\begin{tabular}{|c|c|c|c|c|c|}
\hline Peak No. & Peak ID & Ret Time & Height & Area & Conc. \\
\hline 1 & & 18.657 & 232567.641 & 8154859.000 & 51.3596 \\
\hline 2 & & 22.057 & 209015.578 & 7723116.000 & 48.6404 \\
\hline Total & & & 441583.219 & 15877975.000 & 100.000 \\
\hline
\end{tabular}


(R)-4-ethoxy-3-(4-methoxyphenyl)-3-(p-tolyl)-2,3-dihydro-1,2,5-thiadiazole 1,1-dioxide (5h)<smiles>CCOC1=NS(=O)(=O)NC1(c1ccc(C)cc1)c1ccc(OC)cc1</smiles>

Yellow oil, 93\% yield, 98\% ee;

$[\alpha]_{\mathrm{D}}{ }^{25}=-18.1\left(c 0.35, \mathrm{CHCl}_{3}\right) ;{ }^{1} \mathrm{H}$ NMR $\left(300 \mathrm{MHz}, \mathrm{CDCl}_{3}\right) \delta 7.39-7.23$

(m, 4H), $7.18(\mathrm{~d}, J=8.1 \mathrm{~Hz}, 2 \mathrm{H}), 6.88(\mathrm{~d}, J=8.9 \mathrm{~Hz}, 2 \mathrm{H}), 4.98(\mathrm{~s}, 1 \mathrm{H})$, 4.49 (q, $J=7.1 \mathrm{~Hz}, 2 \mathrm{H}), 3.80(\mathrm{~s}, 3 \mathrm{H}), 2.36(\mathrm{~s}, 3 \mathrm{H}), 1.36(\mathrm{t}, J=7.1 \mathrm{~Hz}$, $3 \mathrm{H}) ;{ }^{13} \mathrm{C}$ NMR $\left(150 \mathrm{MHz}, \mathrm{CDCl}_{3}\right) \delta 177.6,160.2,139.4,135.6,130.2$, 129.7, 129.3, 127.7, 114.2, 70.0, 55.5, 21.2, 13.9; IR (KBr) v 3260, 1611, 1511, 1377, 1326, 1254, $1166 \mathrm{~cm}^{-1}$; HRMS (ESI) for $\mathrm{C}_{18} \mathrm{H}_{20} \mathrm{O}_{4} \mathrm{~N}_{2} \mathrm{NaS}[\mathrm{M}+\mathrm{Na}]^{+}$calcd 383.1041, found 383.1050 .

HPLC: Chiralpak IC column $(250 \mathrm{~mm})$; detected at $220 \mathrm{~nm}$; hexane $/ i$-propanol $=70 / 30$; flow $=$ $0.7 \mathrm{~mL} / \mathrm{min}$; Retention time: $48.2 \mathrm{~min}, 64.6 \mathrm{~min}$ (major).

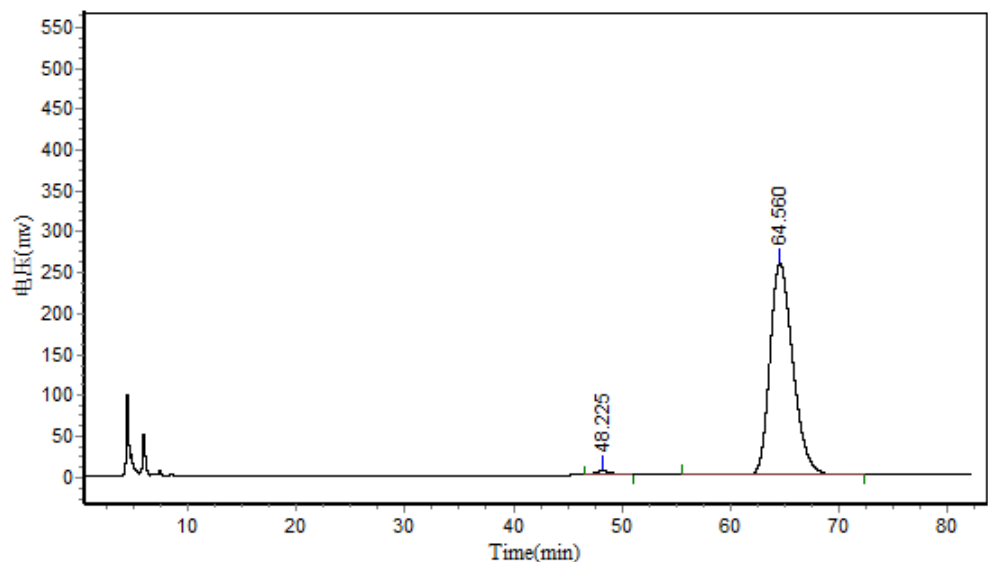

\begin{tabular}{|c|c|c|c|c|c|}
\hline \multicolumn{6}{|c|}{ Results } \\
\hline Peak No. & Peak ID & Ret Time & Height & Area & Conc. \\
\hline 1 & & 48.225 & 4744.440 & 485887.094 & 1.2643 \\
\hline 2 & & 64.560 & 258665.141 & 37944724.000 & 98.7357 \\
\hline Total & & & 263409.581 & 38430611.094 & 100.0000 \\
\hline
\end{tabular}

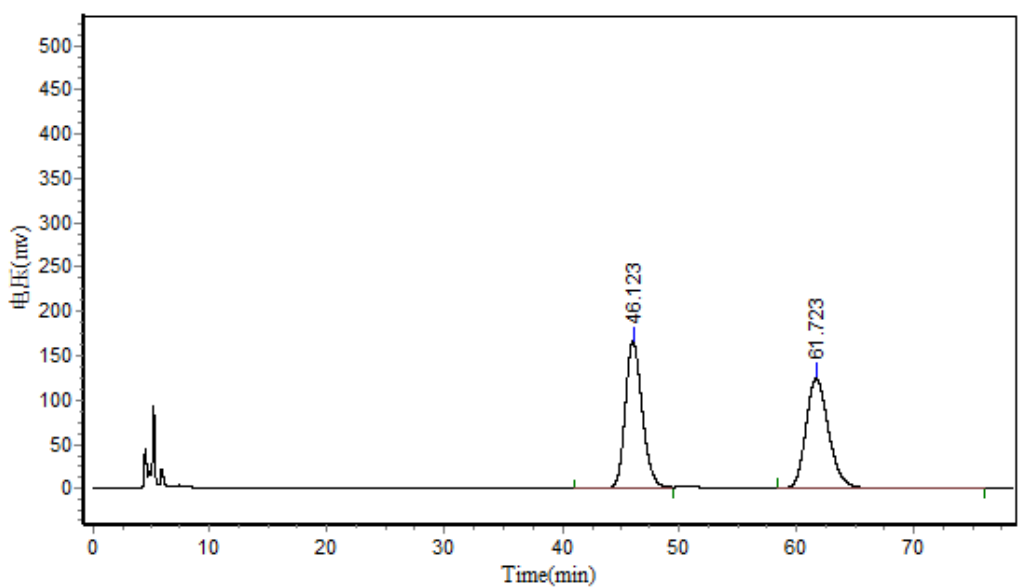

Results

\begin{tabular}{cccccc} 
Peak No. & Peak ID & Ret Time & Height & Area & Conc. \\
\hline 1 & 46.123 & 164840.500 & 16651776.000 & 50.0837 \\
2 & 61.723 & 123342.094 & 16596098.000 & 49.9163 \\
\hline Total & & 288182.594 & 33247874.000 & 100.0000
\end{tabular}




\section{(S)-3-(4-chlorophenyl)-4-ethoxy-3-(4-methoxyphenyl)-2,3-dihydro-1,2,5-thiadiazole}

\section{1,1-dioxide (5i)}<smiles>CCOC1=NS(=O)(=O)NC1(c1ccc(Cl)cc1)c1ccc(OC)cc1</smiles>

Yellow oil, 94\% yield, 98\% ee;

$[\alpha]_{\mathrm{D}}{ }^{25}=+56.0\left(c 0.425, \mathrm{CHCl}_{3}\right) ;{ }^{1} \mathrm{H} \mathrm{NMR}\left(300 \mathrm{MHz}, \mathrm{CDCl}_{3}\right) \delta 7.44(\mathrm{~d}, J$ $=8.8 \mathrm{~Hz}, 2 \mathrm{H}), 7.36(\mathrm{~d}, J=8.8 \mathrm{~Hz}, 2 \mathrm{H}), 7.19(\mathrm{~d}, J=8.9 \mathrm{~Hz}, 2 \mathrm{H}), 6.89(\mathrm{~d}$, $J=8.9 \mathrm{~Hz}, 2 \mathrm{H}), 5.05(\mathrm{~s}, 1 \mathrm{H}), 4.51(\mathrm{pd}, J=7.5,3.3 \mathrm{~Hz}, 2 \mathrm{H}), 3.82(\mathrm{~s}, 3 \mathrm{H})$, $1.37(\mathrm{t}, J=7.1 \mathrm{~Hz}, 3 \mathrm{H}) ;{ }^{13} \mathrm{C} \mathrm{NMR}\left(150 \mathrm{MHz}, \mathrm{CDCl}_{3}\right) \delta 176.9,160.4$, $136.4,135.4,130.3,129.5,129.1,129.0,114.6,70.3,55.6,13.9$; IR (KBr) $v$ 3253, 1611, 1512, 1379, 1327, 1256, 1167, 1093, 1013, $831 \mathrm{~cm}^{-1}$; HRMS (ESI) for $\mathrm{C}_{17} \mathrm{H}_{17} \mathrm{O}_{4} \mathrm{~N}_{2} \mathrm{NaSCl}[\mathrm{M}+\mathrm{Na}]^{+}$calcd 403.0495, found 403.0496.

HPLC: Chiralpak IC column $(250 \mathrm{~mm})$; detected at $220 \mathrm{~nm}$; hexane $/ i$-propanol $=70 / 30$; flow $=$ $0.7 \mathrm{~mL} / \mathrm{min}$; Retention time: $43.4 \mathrm{~min}, 47.1 \mathrm{~min}$ (major).

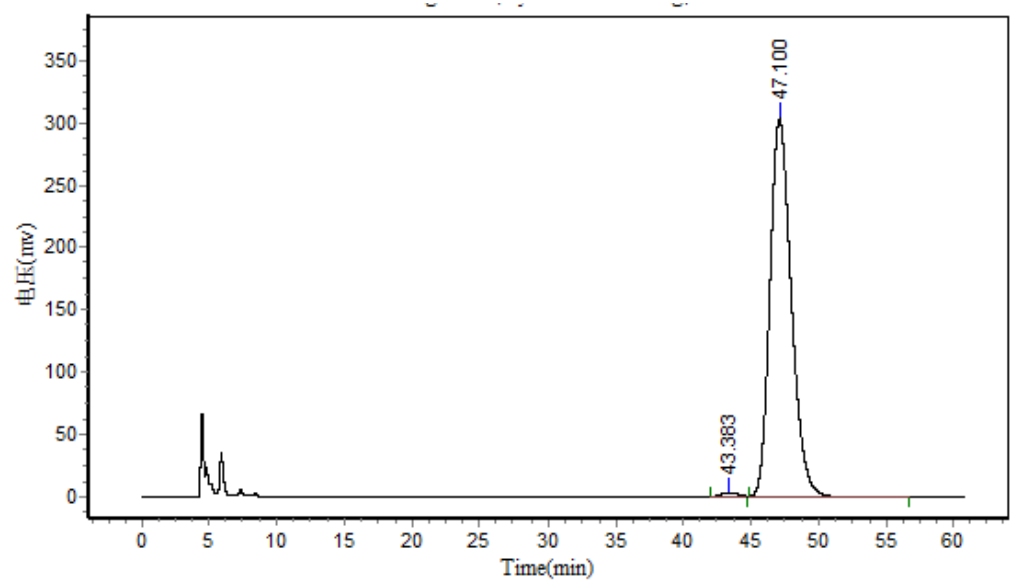

\begin{tabular}{cccccc} 
& \multicolumn{2}{c}{ Results } & Area & Conc. \\
\hline Peak No. & Peak ID & Ret Time & Height & 268542.406 & 0.8034 \\
2 & 43.383 & 3260.420 & 33159278.000 & 99.1966 \\
\hline Total & 47.100 & 302701.875 & 33427820.406 & 100.0000
\end{tabular}

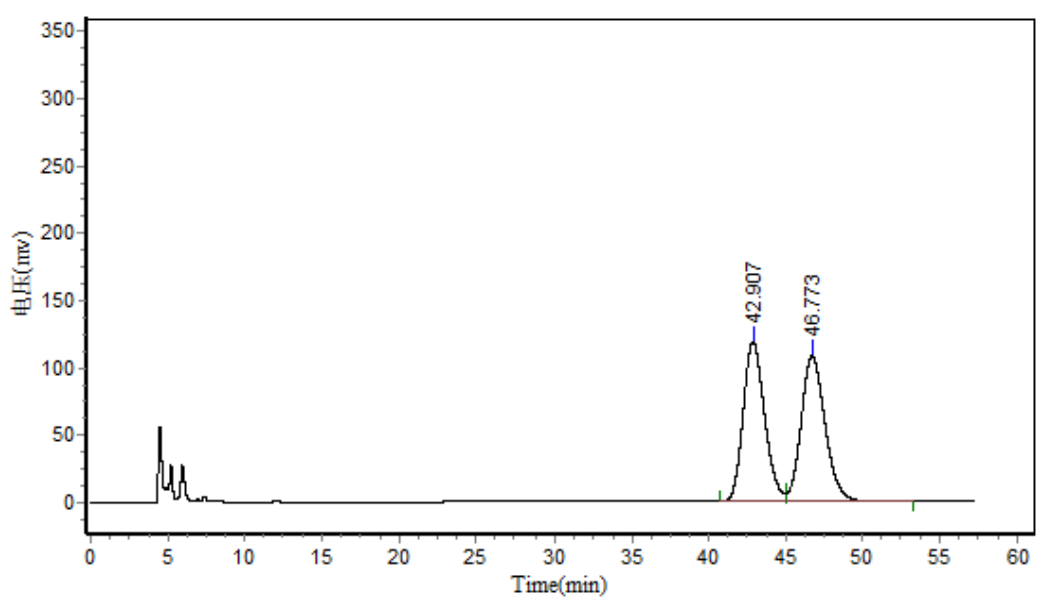

Results

\begin{tabular}{|c|c|c|c|c|c|}
\hline \multicolumn{6}{|c|}{ 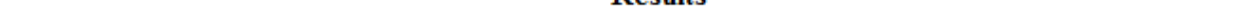 } \\
\hline Peak No. & Peak ID & Ret Time & Height & Area & Conc. \\
\hline 1 & & 42.907 & 118259.945 & 11533699.000 & 49.7396 \\
\hline 2 & & 46.773 & 108139.734 & 11654478.000 & 50.2604 \\
\hline Total & & & 226399.680 & 23188177.000 & 100.0000 \\
\hline
\end{tabular}


(S)-3-(4-chlorophenyl)-4-ethoxy-3-(4-fluorophenyl)-2,3-dihydro-1,2,5-thiadiazole 1,1-dioxide $(5 \mathbf{j})$<smiles>CCOC1=NS(=O)(=O)NC1(c1ccc(F)cc1)c1ccc(Cl)cc1</smiles>

White solid, $95 \%$ yield, $98 \%$ ee; $\mathrm{mp} 94-97{ }^{\circ} \mathrm{C}$.

$[\alpha]_{\mathrm{D}}{ }^{25}=+18.3\left(c 0.56, \mathrm{CHCl}_{3}\right) ;{ }^{1} \mathrm{H}$ NMR $\left(300 \mathrm{MHz}, \mathrm{CDCl}_{3}\right) \delta 7.46-7.21$ $(\mathrm{m}, 6 \mathrm{H}), 7.08(\mathrm{t}, J=8.6 \mathrm{~Hz}, 2 \mathrm{H}), 5.28(\mathrm{~s}, 1 \mathrm{H}), 4.52(\mathrm{q}, J=7.1 \mathrm{~Hz}, 2 \mathrm{H}), 1.38$ $(\mathrm{t}, J=7.1 \mathrm{~Hz}, 3 \mathrm{H}) ;{ }^{13} \mathrm{C}$ NMR $\left(125 \mathrm{MHz}, \mathrm{CDCl}_{3}\right) \delta 176.4,163.3\left(\mathrm{~d},{ }^{1} J_{\mathrm{CF}}=\right.$ $248.8 \mathrm{~Hz}), 136.4,135.8,133.8,129.9\left(\mathrm{~d},{ }^{3} J=8.5 \mathrm{~Hz}\right), 129.3\left(\mathrm{~d},{ }^{4} J=1.8 \mathrm{~Hz}\right)$, $116.2\left(\mathrm{~d},{ }^{2} J_{\mathrm{CF}}=21.3 \mathrm{~Hz}\right), 70.5,13.9 ;{ }^{19} \mathrm{~F}$ NMR $\left(471 \mathrm{MHz}, \mathrm{CDCl}_{3}\right) \delta-111.3$ (d, $J=4.71 \mathrm{~Hz})$; IR (KBr) v 3250, 1611, 1510, 1492, 1375, 1322, 1234, 1158, 1094, 1065, 1013, $835 \mathrm{~cm}^{-1}$; HRMS (ESI) for $\mathrm{C}_{16} \mathrm{H}_{14} \mathrm{O}_{3} \mathrm{~N}_{2} \mathrm{NaSClF}[\mathrm{M}+\mathrm{Na}]^{+}$calcd 391.0295, found 391.0292 .

HPLC: Chiralpak IC column $(250 \mathrm{~mm})$; detected at $220 \mathrm{~nm}$; hexane $/ i$-propanol $=70 / 30$; flow $=$ $0.7 \mathrm{~mL} / \mathrm{min}$; Retention time: $20.0 \mathrm{~min}$ (major), $24.6 \mathrm{~min}$;

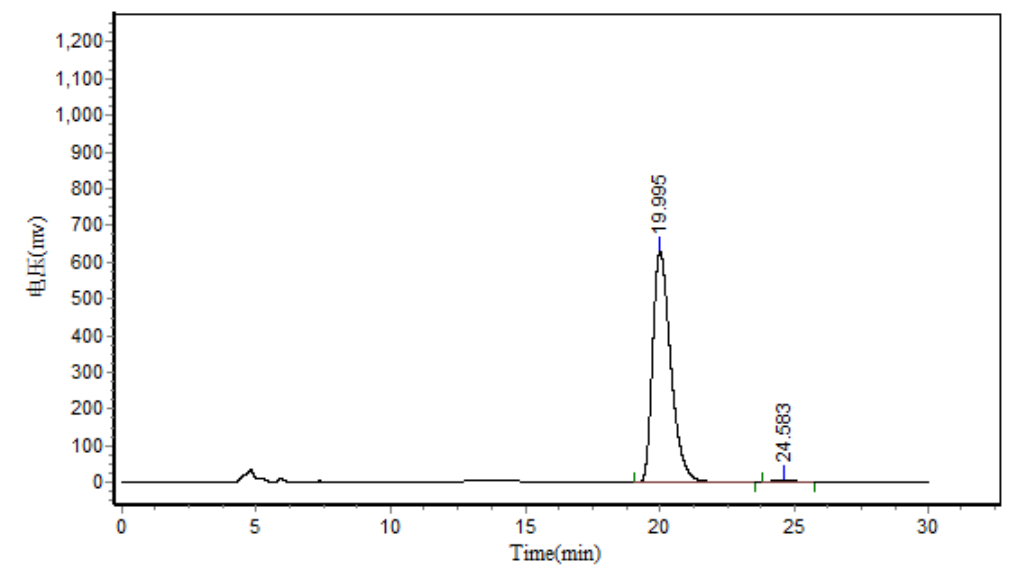

\begin{tabular}{|c|c|c|c|c|c|}
\hline \multicolumn{6}{|c|}{ Results } \\
\hline Peak No. & Peak ID & Ret Time & Height & Area & Conc. \\
\hline 1 & & 19.995 & 629435.750 & 29603264.000 & 98.8110 \\
\hline 2 & & 24.583 & 6827.090 & 356227.156 & 1.1890 \\
\hline Total & & & 636262.840 & 29959491.156 & 100.0000 \\
\hline
\end{tabular}

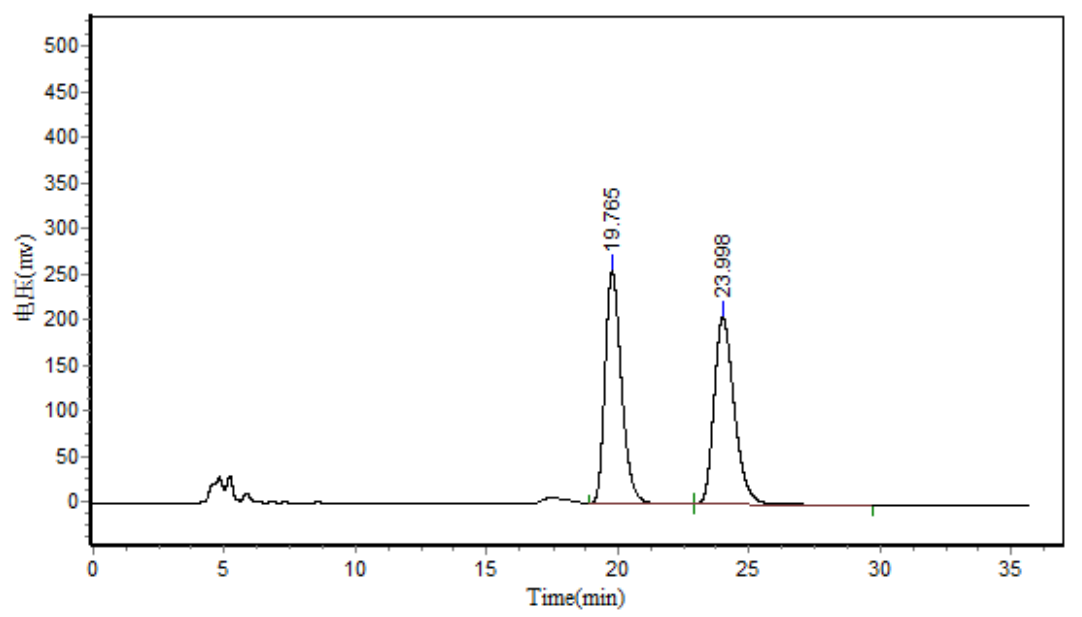

Results

\begin{tabular}{|c|c|c|c|c|c|}
\hline Peak No. & Peak ID & Ret Time & Height & Area & Conc. \\
\hline 1 & & 19.765 & 255264.453 & 11258032.000 & 50.3628 \\
\hline 2 & & 23.998 & 203692.406 & 11095817.000 & 49.6372 \\
\hline Total & & & 458956.859 & 22353849.000 & 100.0000 \\
\hline
\end{tabular}




\section{(S)-3-(3-chlorophenyl)-4-ethoxy-3-(4-methoxyphenyl)-2,3-dihydro-1,2,5-thiadiazole}

\section{1,1-dioxide (5k)}

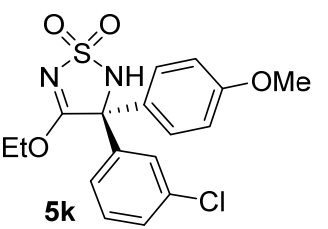

Yellow oil, 96\% yield, 97\% ee;

$[\alpha]_{\mathrm{D}}^{25}=+39.8\left(c 0.405, \mathrm{CHCl}_{3}\right) ;{ }^{1} \mathrm{H}$ NMR $\left(300 \mathrm{MHz}, \mathrm{CDCl}_{3}\right) \delta 7.58-$ $7.29(\mathrm{~m}, 4 \mathrm{H}), 7.20(\mathrm{~d}, J=8.5 \mathrm{~Hz}, 2 \mathrm{H}), 6.89(\mathrm{~d}, J=8.5 \mathrm{~Hz}, 2 \mathrm{H}), 5.05(\mathrm{~s}$, $1 \mathrm{H}), 4.52(\mathrm{dt}, J=6.3,4.8 \mathrm{~Hz}, 2 \mathrm{H}), 3.82(\mathrm{~s}, 3 \mathrm{H}), 1.38(\mathrm{t}, J=7.0 \mathrm{~Hz}, 3 \mathrm{H})$;

${ }^{13} \mathrm{C}$ NMR $\left(150 \mathrm{MHz}, \mathrm{CDCl}_{3}\right) \delta 176.7,160.5,139.9,134.9,130.1,129.5$, 129.1, 128.3, 126.2, 114.6, 70.4, 55.6, 13.9; IR (KBr) v 3449, 1613, 1512, 1467, 1376, 1328, 1255 , $1166 \mathrm{~cm}^{-1}$; HRMS (ESI) for $\mathrm{C}_{17} \mathrm{H}_{17} \mathrm{O}_{4} \mathrm{~N}_{2} \mathrm{NaSCl}[\mathrm{M}+\mathrm{Na}]^{+}$calcd 403.0495, found 403.0496 .

HPLC: Chiralpak IC column $(250 \mathrm{~mm})$; detected at $220 \mathrm{~nm}$; hexane $/ i$-propanol $=70 / 30$; flow $=$ $0.7 \mathrm{~mL} / \mathrm{min}$; Retention time: $30.0 \mathrm{~min}, 36.0 \mathrm{~min}$ (major);

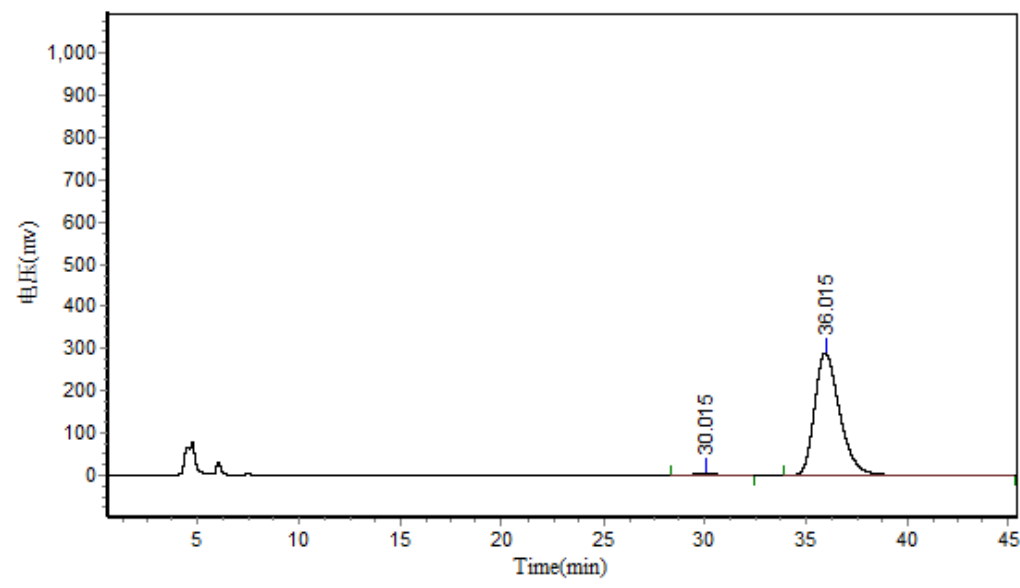

Results

\begin{tabular}{|c|c|c|c|c|c|}
\hline Peak No. & Peak ID & Ret Time & Height & Area & Conc. \\
\hline 1 & & 30.015 & 5191.839 & 377646.500 & 1.4835 \\
\hline 2 & & 36.015 & 289004.031 & 25078916.000 & 98.5165 \\
\hline Total & & & 294195.870 & 25456562.500 & 100.0000 \\
\hline
\end{tabular}

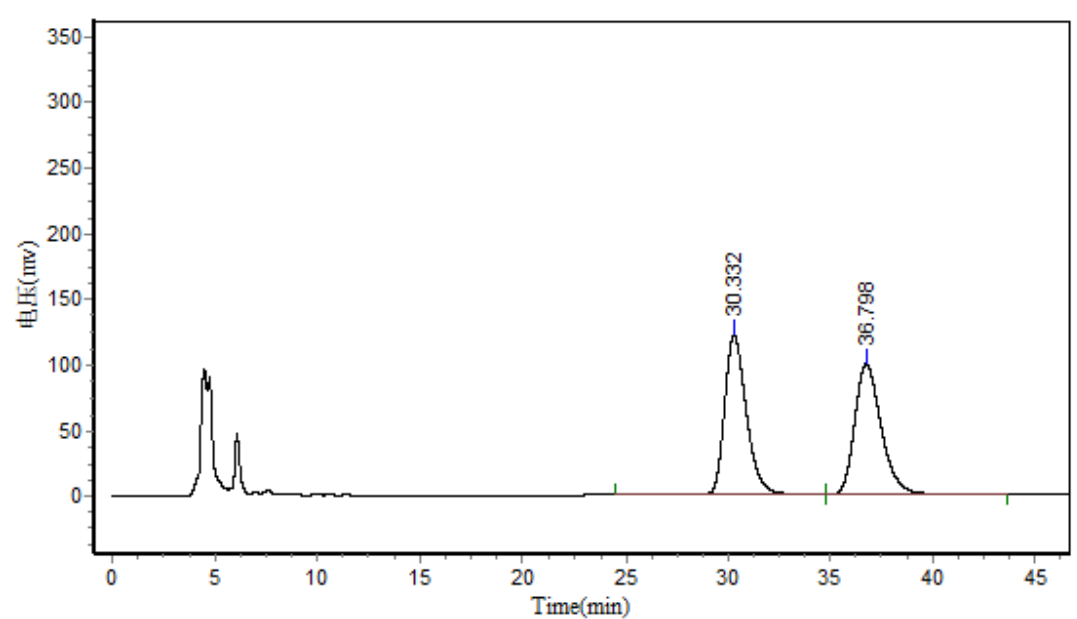

Results

\begin{tabular}{|c|c|c|c|c|c|}
\hline Peak No. & Peak ID & Ret Time & Height & Area & Conc. \\
\hline 1 & & 30.332 & 121063.430 & 9090322.000 & 50.2837 \\
\hline 2 & & 36.798 & 99303.820 & 8987749.000 & 49.7163 \\
\hline Total & & & 220367.250 & 18078071.000 & $100.000 \mathrm{C}$ \\
\hline
\end{tabular}


(S)-3-(3-chlorophenyl)-4-ethoxy-3-(4-fluorophenyl)-2,3-dihydro-1,2,5-thiadiazole 1,1-dioxide (5l)<smiles>CCOC1=NS(=O)(=O)NC1c1ccc(F)cc1</smiles>

White solid, $98 \%$ yield, $97 \%$ ee; $\mathrm{mp} 114-116^{\circ} \mathrm{C}$.

$[\alpha]_{\mathrm{D}}{ }^{25}=+4.9\left(c 0.6, \mathrm{CHCl}_{3}\right) ;{ }^{1} \mathrm{H}$ NMR $\left(300 \mathrm{MHz}, \mathrm{CDCl}_{3}\right) \delta 7.48-7.27$ (m, 6H), $7.09(\mathrm{t}, J=8.6 \mathrm{~Hz}, 2 \mathrm{H}), 4.94(\mathrm{~s}, 1 \mathrm{H}), 4.54(\mathrm{q}, J=7.1 \mathrm{~Hz}, 2 \mathrm{H})$, $1.39(\mathrm{t}, J=7.1 \mathrm{~Hz}, 3 \mathrm{H}) ;{ }^{13} \mathrm{C}$ NMR $\left(150 \mathrm{MHz}, \mathrm{CDCl}_{3}\right) \delta 176.2,163.3(\mathrm{~d}$, $\left.{ }^{1} J_{\mathrm{CF}}=249.0 \mathrm{~Hz}\right), 139.9,135.2,133.6\left(\mathrm{~d},{ }^{4} J=3.1 \mathrm{~Hz}\right), 130.4,129.9\left(\mathrm{~d},{ }^{3} J=\right.$ 6.6 Hz), 128.1, 126.0, $116.3\left(\mathrm{~d},{ }^{2} J_{\mathrm{CF}}=21.0 \mathrm{~Hz}\right), 70.6,13.9 ;{ }^{19} \mathrm{~F} \mathrm{NMR}\left(471 \mathrm{MHz}, \mathrm{CDCl}_{3}\right) \delta-111.3$ (s); IR (KBr) v 3290, 3272, 1618, 1510, 1377, 1324, 1241, 1178, 1165, $952 \mathrm{~cm}^{-1}$; HRMS (ESI) for $\mathrm{C}_{16} \mathrm{H}_{14} \mathrm{O}_{3} \mathrm{~N}_{2} \mathrm{NaSClF}[\mathrm{M}+\mathrm{Na}]^{+}$calcd 391.0295, found 391.0301.

HPLC: Chiralpak AD-H column $(250 \mathrm{~mm})$; detected at $220 \mathrm{~nm}$; hexane $/ i$-propanol $=80 / 20$; flow $=0.7 \mathrm{~mL} / \mathrm{min}$; Retention time: $10.1 \mathrm{~min}, 15.8 \mathrm{~min}$ (major).

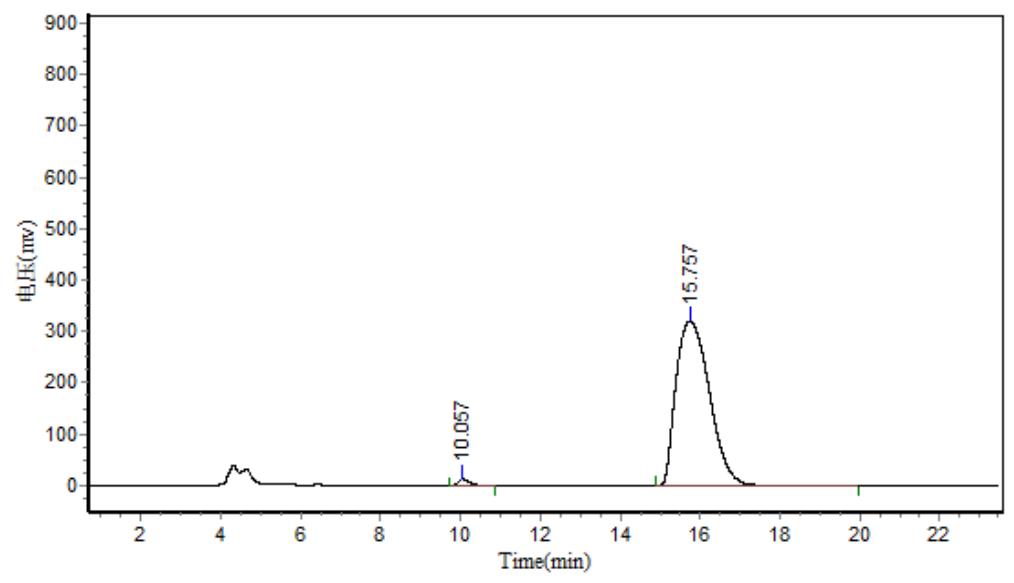

\begin{tabular}{|c|c|c|c|c|c|}
\hline \multicolumn{6}{|c|}{ Results } \\
\hline Peak No. & Peak ID & Ret Time & Height & Area & Conc. \\
\hline$\overline{1}$ & & 10.057 & 13847.739 & 288338.688 & 1.5174 \\
\hline 2 & & 15.757 & 319498.281 & 18714232.000 & 98.4826 \\
\hline Total & & & 333346.021 & 19002570.688 & 100.0000 \\
\hline
\end{tabular}

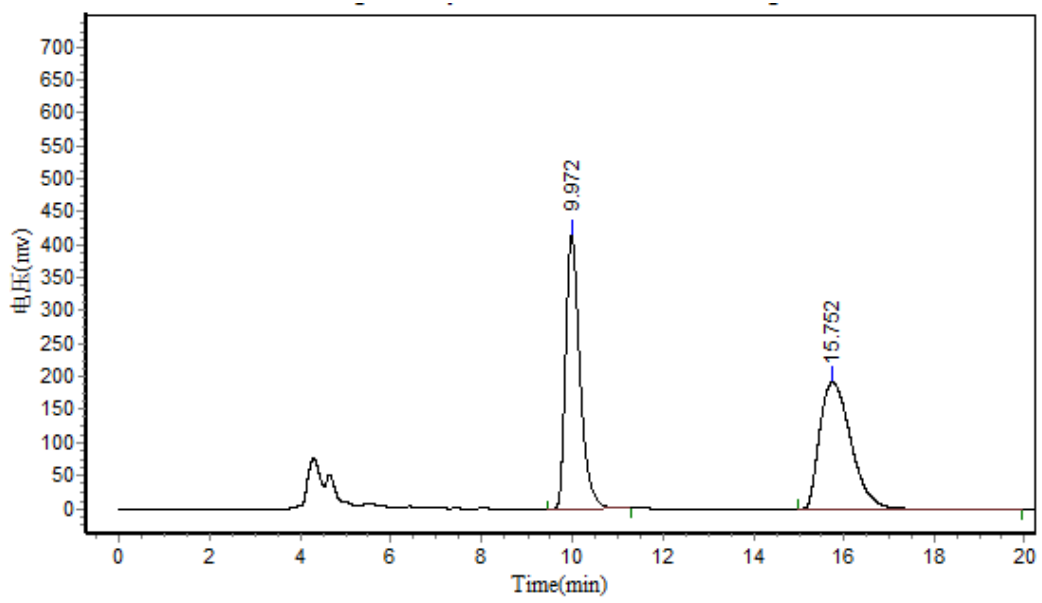

Results

\begin{tabular}{|c|c|c|c|c|c|}
\hline Peak No. & Peak ID & Ret Time & Height & Area & Conc. \\
\hline 1 & & 9.972 & 412801.688 & 9146863.000 & 49.7533 \\
\hline 2 & & 15.752 & 191951.656 & 9237585.000 & 50.2467 \\
\hline Total & & & 604753.344 & 18384448.000 & 100.0000 \\
\hline
\end{tabular}




\section{The synthesis of compound 6}

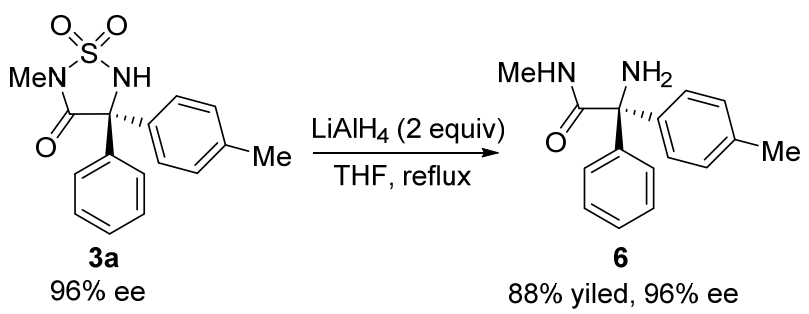

To a suspension of $\mathrm{LiAlH}_{4}(15 \mathrm{mg}, 0.40 \mathrm{mmol}, 2.0$ equiv) in THF $(2 \mathrm{~mL})$, a solution of $3 \mathbf{a}$ (63 mg, $0.20 \mathrm{mmol}, 1.0$ equiv) in THF $(1 \mathrm{~mL})$ was added dropwise at $0{ }^{\circ} \mathrm{C}$. After refluxing for $3 \mathrm{~h}$, the mixture was cooled, quenched with ice water and $10 \% \mathrm{NaOH}$ was then added. The aqueous layer was extracted with EtOAc, washed with brine, dried over $\mathrm{Na}_{2} \mathrm{SO}_{4}$, and concentrated under reduced pressure. The residue was purified by silica gel column chromatography to afford compound 6 ( $45 \mathrm{mg}, 88 \%$ yield).

6: Colorless oil; $[\alpha]_{\mathrm{D}}{ }^{25}=-3.3\left(c 0.15, \mathrm{CHCl}_{3}\right) ;{ }^{1} \mathrm{H}$ NMR $\left(300 \mathrm{MHz}, \mathrm{CDCl}_{3}\right) \delta 7.36(\mathrm{~d}, J=7.9 \mathrm{~Hz}$, 2H), $7.34-7.17(\mathrm{~m}, 5 \mathrm{H}), 7.12(\mathrm{~d}, J=8.0 \mathrm{~Hz}, 2 \mathrm{H}), 2.84(\mathrm{~d}, J=4.9 \mathrm{~Hz}, 3 \mathrm{H}), 2.33(\mathrm{~s}, 3 \mathrm{H}), 2.16(\mathrm{~s}$, $2 \mathrm{H}) ;{ }^{13} \mathrm{C}$ NMR (100 MHz, $\left.\mathrm{CDCl}_{3}\right)$ 174.5, 145.5, 142.3, 137.3, 129.1, 128.4, 127.6, 127.5, 127.4, 68.0, 26.7, 21.2; IR (KBr) v 3432, 2962, 2924, 2854, 1655, 1261, 1098, 1023, $802 \mathrm{~cm}^{-1}$; HRMS (ESI) for $\mathrm{C}_{16} \mathrm{H}_{19} \mathrm{ON} \mathrm{N}_{2}[\mathrm{M}+\mathrm{H}]^{+}$calcd 255.1492, found 255.1490.

HPLC (of its $N$-Boc protected derivative): Chiralpak IC column $(250 \mathrm{~mm})$; detected at $220 \mathrm{~nm}$; hexane $/ i$-propanol $=95 / 5$; flow $=1.0 \mathrm{~mL} / \mathrm{min}$; Retention time: $21.9 \mathrm{~min}$ (major), $25.6 \mathrm{~min}$.

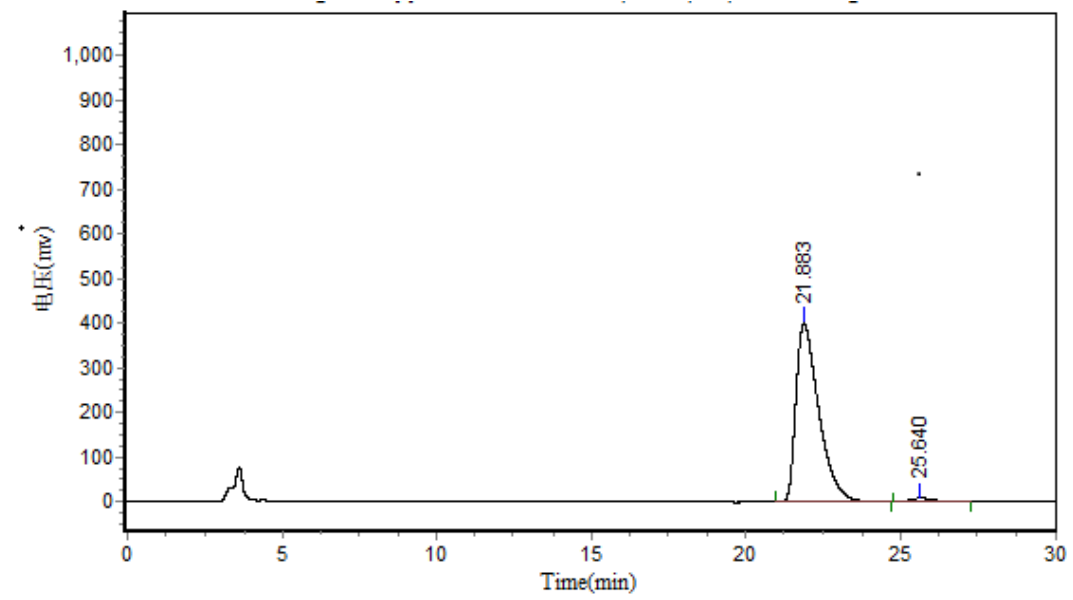

\begin{tabular}{|c|c|c|c|c|c|}
\hline \multicolumn{6}{|c|}{ Results } \\
\hline Peak No. & Peak ID & Ret Time & Height & Area & Conc. \\
\hline$\overline{1}$ & & 21.883 & 399227.094 & 20647586.000 & 97.7258 \\
\hline 2 & & 25.640 & 8752.904 & 480494.125 & 2.2742 \\
\hline Total & & & 407979.998 & 21128080.125 & 100.0000 \\
\hline
\end{tabular}




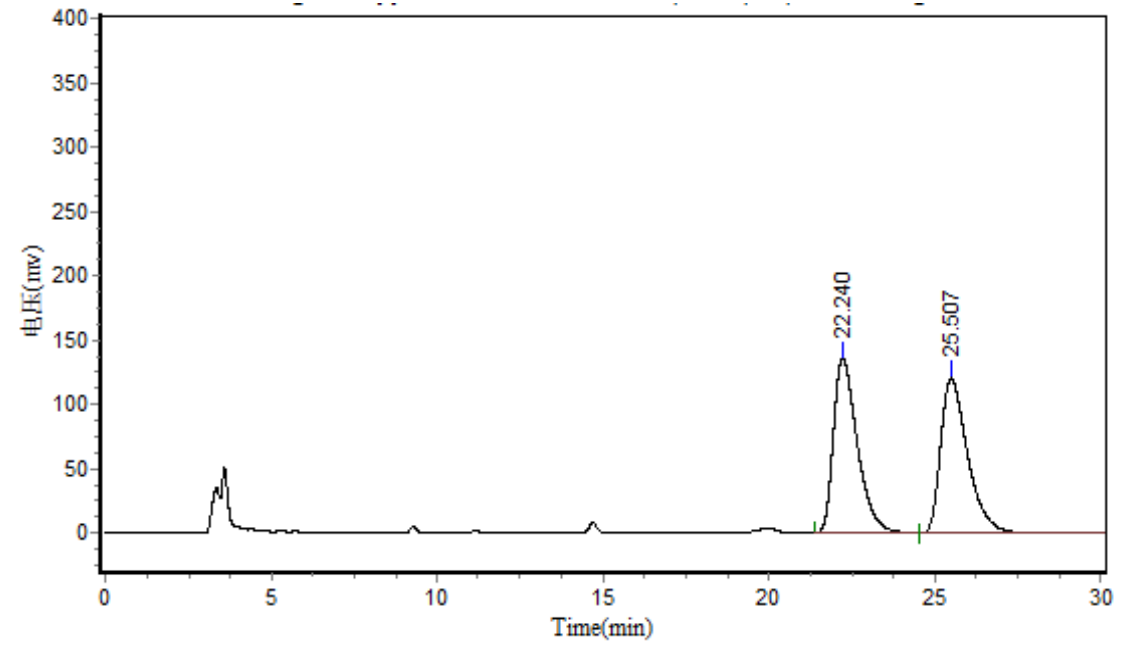

Results

\begin{tabular}{|c|c|c|c|c|c|}
\hline Peak No. & Peak ID & Ret Time & Height & Area & Conc. \\
\hline 1 & & 22.240 & 136489.625 & 6891697.000 & 49.9167 \\
\hline 2 & & 25.507 & 120474.695 & 6914708.000 & 50.0833 \\
\hline Total & & & 256964.320 & 13806405.000 & 100.0000 \\
\hline
\end{tabular}




\section{The synthesis of compounds 7-10.}

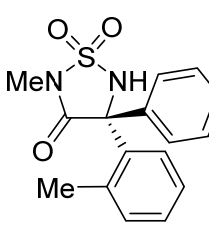

3k

$85 \%$ ee $\stackrel{(a)}{\longrightarrow}$<smiles>CN1C(=O)[C@]2(c3ccccc3)c3ccccc3CN2S1(=O)=O</smiles>

7

$82 \%$ yield, $85 \%$ ee recrystallization: $60 \%$ yield, $99 \%$ ee $\stackrel{(b)}{\longrightarrow}$

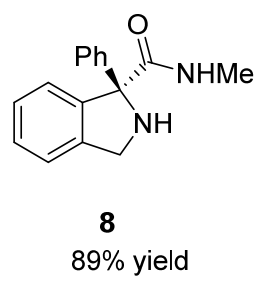

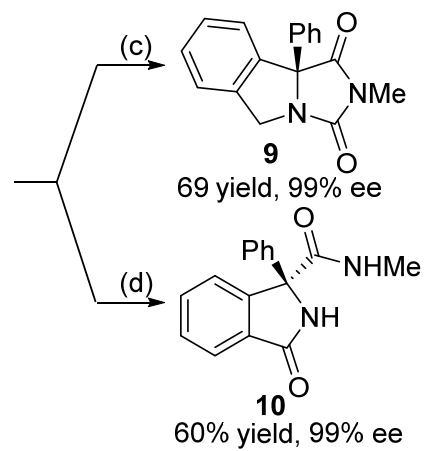

$60 \%$ yield, $99 \%$ ee

(a) NBS (1 equiv), AIBN (10 mol\%), $\mathrm{CCl}_{4}$, reflux. (b) Na naphthalene (5 equiv), DME, $-78^{\circ} \mathrm{C}$. (c)

Triphosgene, $\mathrm{Et}_{3} \mathrm{~N}, \mathrm{THF}, 0^{\circ} \mathrm{C}$ to rt. (d) $\mathrm{ZnBr}_{2}, \mathrm{TBHP}$, pyridine, $100^{\circ} \mathrm{C}$.

(a) Compound 3k (63 mg, $0.2 \mathrm{mmol}, 1.0$ equiv), NBS (36 mg, 1.0 equiv), and AIBN (0.02 mmol, 0.1 equiv) were dissolved in $3 \mathrm{~mL}$ of $\mathrm{CCl}_{4}$ and heated to reflux. The reaction was monitored by TLC, and later quenched by aqueous $\mathrm{Na}_{2} \mathrm{CO}_{3}$. The mixture was extracted with EtOAc and washed with brine, dried over $\mathrm{Na}_{2} \mathrm{SO}_{4}$, and concentrated under reduced pressure. The residue was purified by silica gel column chromatography to afford compound 7 (52 $\mathrm{mg}, 82 \%$ yield, 85\% ee). The enantioselectivity could be improved to $99 \%$ ee by recrystallization from EA/PE.

7: White solid, mp $115-118^{\circ} \mathrm{C} ;[\alpha]_{\mathrm{D}}{ }^{25}=-104.8\left(c 0.44, \mathrm{CHCl}_{3}\right) ;{ }^{1} \mathrm{H}_{\mathrm{NMR}}\left(300 \mathrm{MHz}, \mathrm{CDCl}_{3}\right) \delta$ $7.76-7.69$ (m, 2H), $7.68-7.63(\mathrm{~m}, 1 \mathrm{H}), 7.45-7.31(\mathrm{~m}, 5 \mathrm{H}), 7.28(\mathrm{~d}, J=6.3 \mathrm{~Hz}, 1 \mathrm{H}), 5.29(\mathrm{~d}, J$ $=14.9 \mathrm{~Hz}, 1 \mathrm{H}), 4.85(\mathrm{~d}, J=14.9 \mathrm{~Hz}, 1 \mathrm{H}), 3.15(\mathrm{~s}, 3 \mathrm{H}) ;{ }^{13} \mathrm{C} \mathrm{NMR}\left(100 \mathrm{MHz}, \mathrm{CDCl}_{3}\right) \delta 167.3$, 137.9, 137.1, 135.7, 129.9, 129.1, 129.0, 128.8, 126.1, 124.5, 122.6, 83.4, 55.2, 26.4; IR (KBr) $v$ 3422, 2962, 2919, 2852, 1742, 1375, 1181, 1063, $1042 \mathrm{~cm}^{-1}$; HRMS (EI) for $\mathrm{C}_{16} \mathrm{H}_{14} \mathrm{O}_{3} \mathrm{~N}_{2} \mathrm{~S}[\mathrm{M}]^{+}$ calcd 314.0725, found 314.0721 .

HPLC for compound 7: Chiralcel OZ-H column (250 mm); detected at $220 \mathrm{~nm}$; hexane/i-propanol =95/5; flow $=1 \mathrm{~mL} / \mathrm{min}$; Retention time: $9.7 \mathrm{~min}$ (major), $12.4 \mathrm{~min}$.

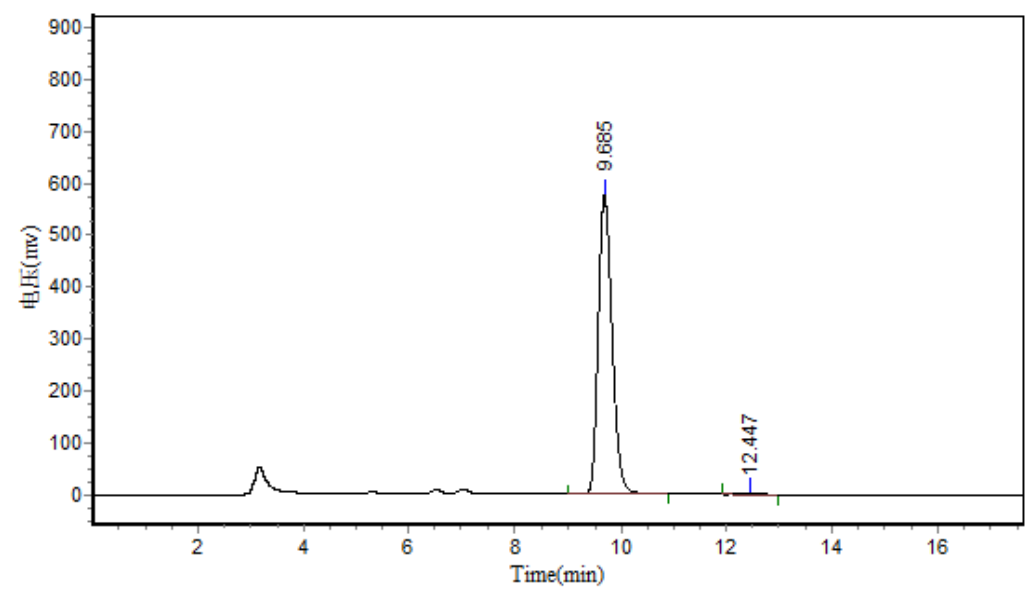

\begin{tabular}{|c|c|c|c|c|c|}
\hline \multicolumn{6}{|c|}{ Results } \\
\hline Peak No. & Peak ID & Ret Time & Height & Area & Conc. \\
\hline$\overline{1}$ & & 9.685 & 575437.375 & 10585601.000 & 99.6849 \\
\hline 2 & & 12.447 & 1392.781 & 33458.750 & 0.3151 \\
\hline Total & & & 576830.156 & 10619059.750 & 100.0000 \\
\hline
\end{tabular}




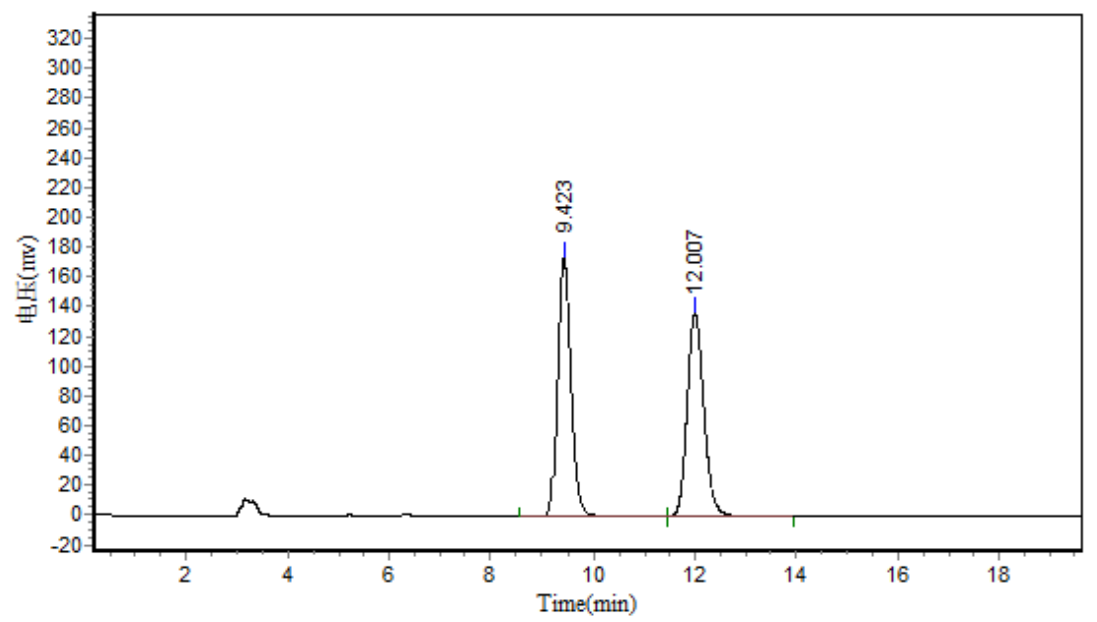

\begin{tabular}{cccccc} 
& \multicolumn{2}{c}{ Results } & Area & Conc. \\
\hline Peak No. & Peak ID & Ret Time & Height & 3129718.000 & 49.9078 \\
2 & 9.423 & 173436.391 & 3141281.250 & 50.0922 \\
\hline Total & 12.007 & 136623.016 & 6270999.250 & 100.0000
\end{tabular}

(b) Compound 7 (52 mg, $0.165 \mathrm{mmol}, 1.0$ equiv) was dissolved in $3 \mathrm{~mL}$ of DME and cooled to $-78{ }^{\circ} \mathrm{C}$ under $\mathrm{N}_{2}$ atmosphere. Sodium naphthalide $(0.5 \mathrm{M}$ in DME, $1.7 \mathrm{~mL}, 5$ equiv) was added dropwise for 30 seconds. After 3 minutes, the reaction was quenched with aqueous $\mathrm{NaCl}$, and diluted with EtOAc. The solvents were evaporated and the residue was purified by silica gel column chromatography to give the desired product 8 ( $37 \mathrm{mg}, 89 \%$ yield).

8: White solid, mp $130-132{ }^{\circ} \mathrm{C} ;{ }^{1} \mathrm{H}$ NMR $\left(300 \mathrm{MHz}, \mathrm{CDCl}_{3}\right) \delta 8.30(\mathrm{~s}, 1 \mathrm{H}), 7.63(\mathrm{~d}, J=6.8 \mathrm{~Hz}$, $1 \mathrm{H}), 7.41-7.19(\mathrm{~m}, 6 \mathrm{H}), 7.12(\mathrm{~d}, J=7.6 \mathrm{~Hz}, 2 \mathrm{H}), 4.37(\mathrm{~d}, J=14.1 \mathrm{~Hz}, 1 \mathrm{H}), 4.23(\mathrm{~d}, J=14.1 \mathrm{~Hz}$, $1 \mathrm{H}), 2.87(\mathrm{~d}, J=5.0 \mathrm{~Hz}, 3 \mathrm{H}), 2.22(\mathrm{~s}, 1 \mathrm{H}) ;{ }^{13} \mathrm{C} \mathrm{NMR}\left(125 \mathrm{MHz}, \mathrm{CDCl}_{3}\right) \delta$ 173.0, 144.3, 141.7, $140.8,128.8,128.4,127.8,127.7,126.8,126.1,122.2,78.0,51.3,26.3$. IR (KBr) v 3354, 3333, 3067, 2949, 2890, 2823,1649, 1518, 1438, 1409, 1084, 755, $695 \mathrm{~cm}^{-1}$; HRMS (ESI) for $\mathrm{C}_{16} \mathrm{H}_{17} \mathrm{ON}_{2}[\mathrm{M}+\mathrm{H}]^{+}$calcd 253.1335, found 253.1336.

(c) To a solution of 8 (37 mg, $0.147 \mathrm{mmol}, 1.0$ equiv) in dry THF, triphosgene (44 mg, 1.0 equiv) was added at $0{ }^{\circ} \mathrm{C}$ slowly, then $\mathrm{Et}_{3} \mathrm{~N}$ was added $(0.1 \mathrm{~mL}, 5$ equiv) dropwisely. The resulting mixture was allowed to stir at room temperature overnight. After completion of reaction, the reaction mixture was added to ice and extracted with ethyl acetate. The organic layer washed with brine, dried over $\mathrm{Na}_{2} \mathrm{SO}_{4}$, and concentrated under reduced pressure. The residue was then purified by silica gel column chromatography to give the desired product 9 (69\% yield, $99 \%$ ee).

9: White solid, $\mathrm{mp} 110-112^{\circ} \mathrm{C}$; $[\alpha]_{\mathrm{D}}{ }^{25}=+112.3\left(c 0.295, \mathrm{CHCl}_{3}\right) ;{ }^{1} \mathrm{H} \mathrm{NMR}\left(300 \mathrm{MHz}, \mathrm{CDCl}_{3}\right) \delta$ $7.78(\mathrm{~d}, J=6.5 \mathrm{~Hz}, 1 \mathrm{H}), 7.56(\mathrm{~d}, J=7.6 \mathrm{~Hz}, 2 \mathrm{H}), 7.43-7.23(\mathrm{~m}, 6 \mathrm{H}), 5.04(\mathrm{~d}, J=15.0 \mathrm{~Hz}, 1 \mathrm{H})$, $4.47(\mathrm{~d}, J=15.0 \mathrm{~Hz}, 1 \mathrm{H}), 3.16(\mathrm{~s}, 3 \mathrm{H}) ;{ }^{13} \mathrm{C}$ NMR $\left(100 \mathrm{MHz}, \mathrm{CDCl}_{3}\right) \delta 157.5,153.7,139.9,139.1$, 138.6, 129.3, 128.9, 128.6, 128.4, 125.8, 123.7, 123.1, 52.0, 35.5; IR (KBr) v 3430, 3080, 3004, 2924, 2865, 1810, 1734, 1459, 1350, 1328, 1008, 987, $915 \mathrm{~cm}^{-1}$; HRMS (EI) for $\mathrm{C}_{17} \mathrm{H}_{14} \mathrm{O}_{2} \mathrm{~N}_{2}$ $[\mathrm{M}]^{+}$calcd 278.1055, found 278.1057;

HPLC for compound 9: Chiralcel OZ-H column $(250 \mathrm{~mm})$; detected at $220 \mathrm{~nm}$; hexane $/ i$-propanol 
$=97 / 3$; flow $=0.7 \mathrm{~mL} / \mathrm{min}$; Retention time: $10.6 \mathrm{~min}$ (major), $12.3 \mathrm{~min}$.

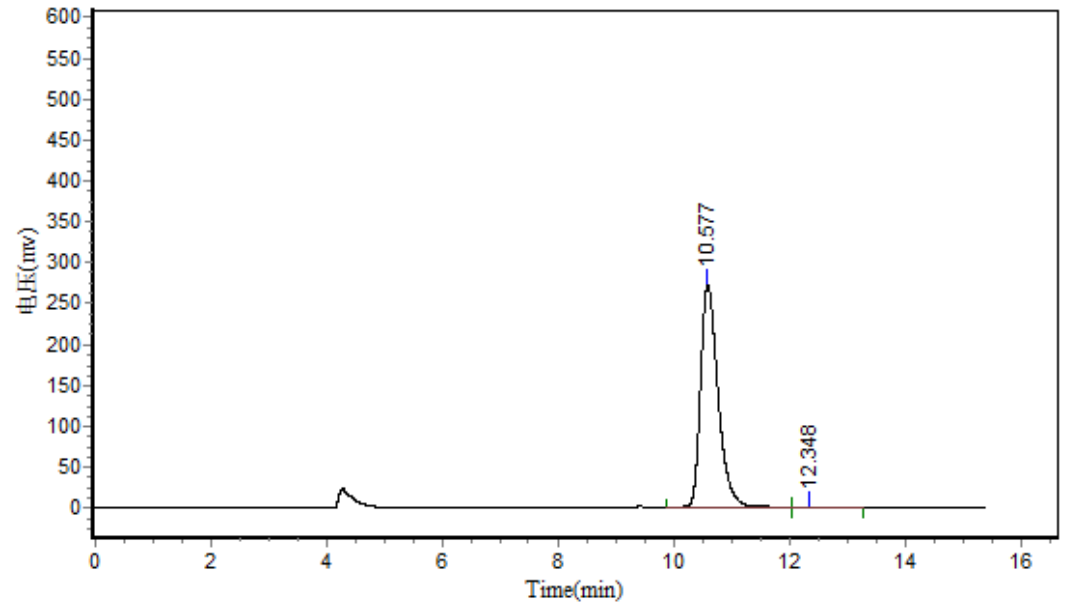

\section{Results}

\begin{tabular}{|c|c|c|c|c|c|}
\hline Peak No. & Peak ID & Ret Time & Height & Area & Conc. \\
\hline 1 & & 10.577 & 273330.719 & 5844332.500 & 99.6212 \\
\hline 2 & & 12.348 & 807.313 & 22223.799 & 0.3788 \\
\hline Total & & & 274138.032 & 5866556.299 & 100.0000 \\
\hline
\end{tabular}

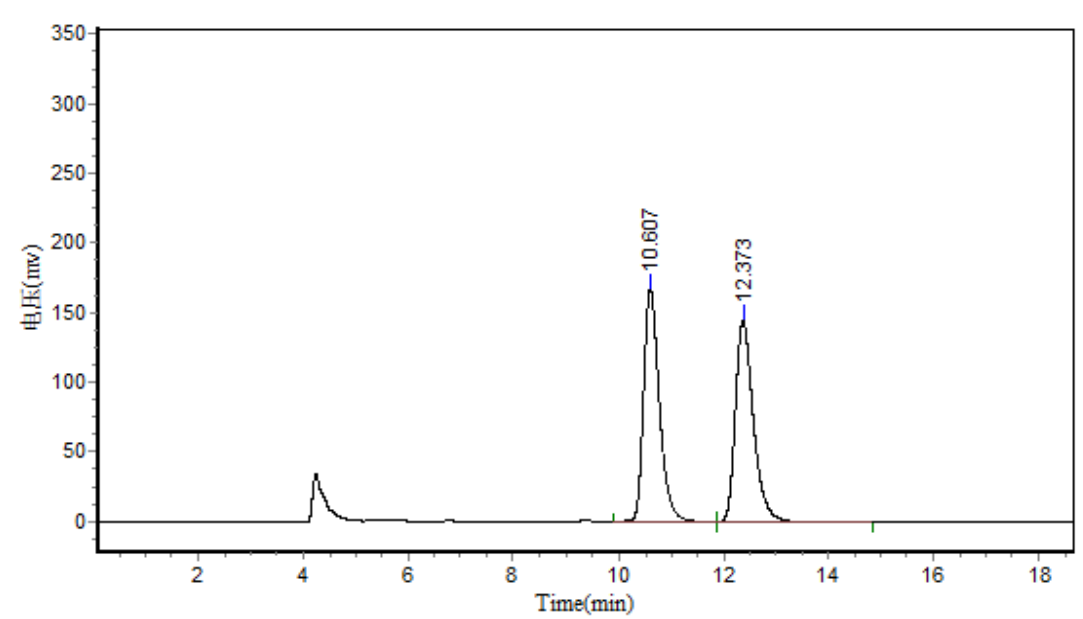

Results

\begin{tabular}{|c|c|c|c|c|c|}
\hline Peak No. & Peak ID & Ret Time & Height & Area & Conc. \\
\hline 1 & & 10.607 & 166852.672 & 3525344.000 & 50.2798 \\
\hline 2 & & 12.373 & 144437.828 & 3486111.000 & 49.7202 \\
\hline Total & & & 311290.500 & 7011455.000 & 100.0000 \\
\hline
\end{tabular}

(d) Compound 8 (59 mg, $0.20 \mathrm{mmol}$ ) and $\mathrm{ZnBr}_{2}$ (2 equiv) were dissolved in pyridine ( $\left.0.1 \mathrm{~mL}\right)$. To the solution was added the tert-butyl hydroperoxide $(0.1 \mathrm{~mL}, 70 \%$ aqueous $)$ and the mixture was stirred at $100{ }^{\circ} \mathrm{C}$ for 3 days. When the reaction was over, a saturated aq. $\mathrm{NaCl}$ was added and the mixture was extracted with EtOAc $(10 \mathrm{~mL} \times 3)$. The combined organic phase was dried over $\mathrm{Na}_{2} \mathrm{SO}_{4}$, filtered, and concentrated. The residue was purified by silica gel flash chromatography to afford the corresponding $\alpha$-arylglycine product 10 (32 $\mathrm{mg}, 60 \%$ yield, $99 \%$ ee).

10: White solid, $\mathrm{mp} 223-226^{\circ} \mathrm{C} ;[\alpha]_{\mathrm{D}}{ }^{25}=+21.0\left(c 0.1, \mathrm{CHCl}_{3}\right) ;{ }^{1} \mathrm{H}$ NMR $\left(300 \mathrm{MHz}, \mathrm{CDCl}_{3}\right) \delta$ 8.06 (s, 1H), 7.84 (d, $J=7.6 \mathrm{~Hz}, 1 \mathrm{H}), 7.77$ (d, $J=7.4 \mathrm{~Hz}, 1 \mathrm{H}), 7.63$ (t, $J=7.6 \mathrm{~Hz}, 1 \mathrm{H}), 7.52$ (t, $J$ 
$=7.4 \mathrm{~Hz}, 1 \mathrm{H}), 7.29(\mathrm{~d}, J=10.8 \mathrm{~Hz}, 5 \mathrm{H}), 7.22(\mathrm{~s}, 1 \mathrm{H}), 2.82(\mathrm{~d}, J=4.7 \mathrm{~Hz}, 3 \mathrm{H}) ;{ }^{13} \mathrm{C} \mathrm{NMR}(125$ $\left.\mathrm{MHz}, \mathrm{CDCl}_{3}\right) \delta 171.5,170.5,146.7,139.1,133.3,130.3,129.5,129.1,128.7,126.6,125.5,123.9$, 71.8, 27.0; IR (KBr) $v$ 3396, 3312, 1746, 1683, 1641, 1535, 1468, 1312, 1274, $691 \mathrm{~cm}^{-1}$; HRMS (ESI) for $\mathrm{C}_{16} \mathrm{H}_{14} \mathrm{O}_{2} \mathrm{~N}_{2} \mathrm{Na}[\mathrm{M}+\mathrm{Na}]^{+}$calcd 289.0947, found 289.0943.

HPLC for compound 10: Chiralpak AD-3 column $(250 \mathrm{~mm})$; detected at $210 \mathrm{~nm}$; hexane $/ i$-propanol $=90 / 10$; flow $=0.7 \mathrm{~mL} / \mathrm{min}$; Retention time: $18.0 \mathrm{~min}$ (major), $27.2 \mathrm{~min}$

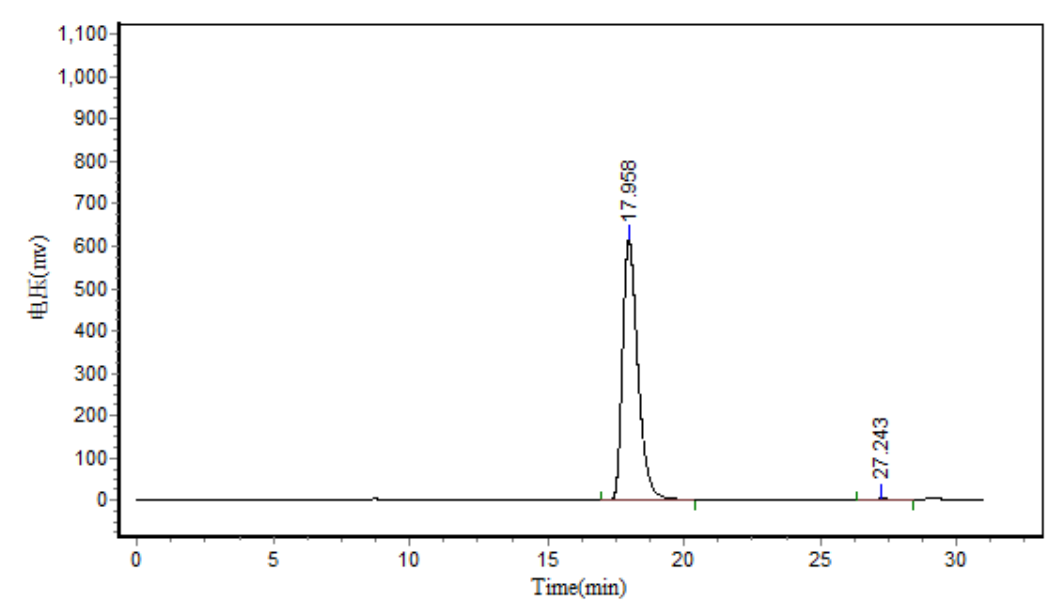

\begin{tabular}{|c|c|c|c|c|c|}
\hline \multicolumn{6}{|c|}{ Results } \\
\hline Peak No. & Peak ID & Ret Time & Height & Area & Conc. \\
\hline 1 & & 17.958 & 613428.500 & 24153456.000 & 99.6188 \\
\hline 2 & & 27.243 & 1615.321 & 92414.547 & 0.3812 \\
\hline Total & & & 615043.821 & 24245870.547 & 100.0000 \\
\hline
\end{tabular}

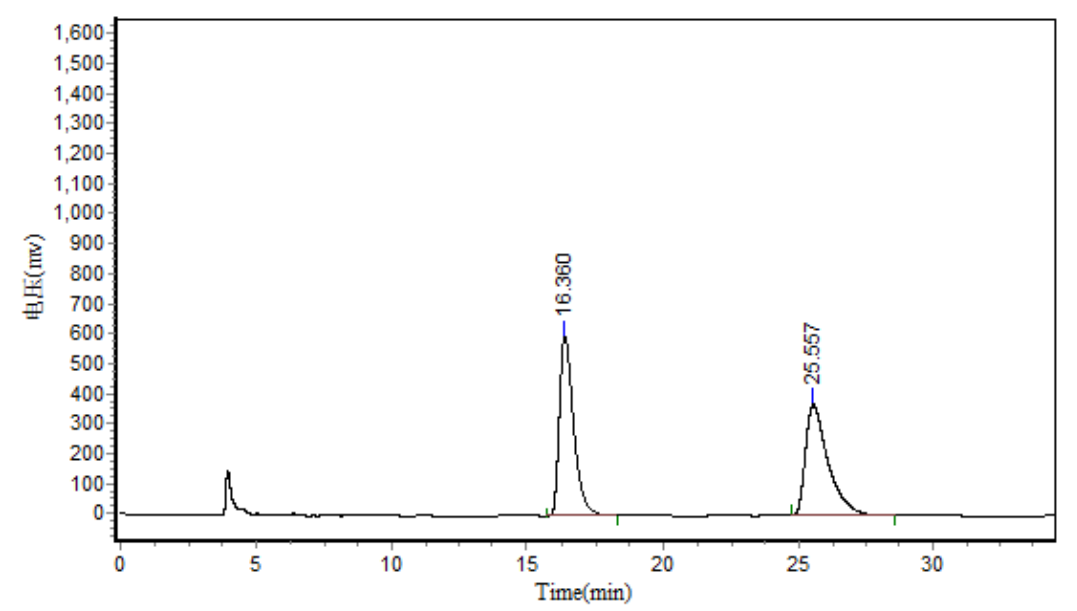

\begin{tabular}{|c|c|c|c|c|c|}
\hline \multicolumn{6}{|c|}{ Results } \\
\hline Peak No. & Peak ID & Ret Time & Height & Area & Conc. \\
\hline 1 & & 16.360 & 603008.688 & 22117230.000 & 50.6644 \\
\hline 2 & & 25.557 & 369429.313 & 21537158.000 & 49.3356 \\
\hline Total & & & 972438.000 & 43654388.000 & 100.0000 \\
\hline
\end{tabular}


9. The synthesis of compound 3r, 11-13.

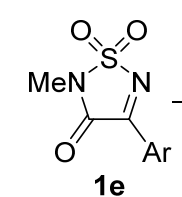

$\left(\mathrm{Ar}=3-\mathrm{BrC}_{6} \mathrm{H}_{4}\right)$ (a) $\mathrm{MeN}^{\mathrm{O}}, \mathrm{S}, \mathrm{O}$<smiles>CNC(=O)C(N)([In])c1ccccc1</smiles>
$3 r$

$98 \%$ yield $93 \%$ ee

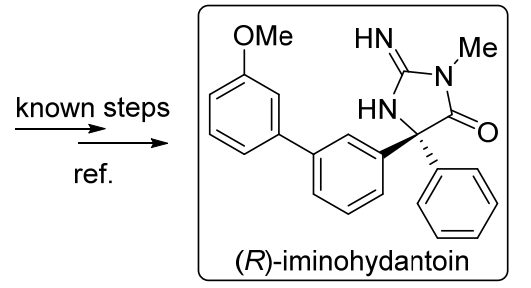

(b) $\mathrm{MeHN} \quad \mathrm{NH}_{2}$

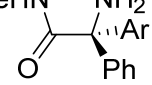

11

$86 \%$ yield<smiles>CN1C(=O)NC(c2ccccc2)([Al](C)(Cl)Cl)C1=O</smiles>

$71 \%$ yield<smiles>CN1C(=O)C([Al])(c2ccccc2)NC1=S</smiles>

$13\left(\mathrm{Ar}=3-\mathrm{BrC}_{6} \mathrm{H}_{4}\right)$ $95 \%$ yield, $93 \%$ ee

BACE1 inhibitor

$\mathrm{K}_{\mathrm{i}:}: 79 \mathrm{~nm}(\mathrm{LE} 0.35)$

cell $\mathrm{A} \beta 40 \mathrm{IC}_{50}: 2.25 \mu \mathrm{M}$

(a) $\mathrm{PhB}(\mathrm{OH})_{2}$ (2 equiv), $\left[\mathrm{Rh}(\mathrm{COE})_{2} \mathrm{Cl}\right]_{2}\left(1.5 \mathrm{~mol} \%\right.$ ), (S)-L11 (3.0 equiv), $\mathrm{K}_{3} \mathrm{PO}_{4}$ (1 equiv), toluene, rt; (b) $\mathrm{LiAlH}_{4}$,

THF, reflux; (c) Triphosgene, $\mathrm{Et}_{3} \mathrm{~N}, \mathrm{THF}, \mathrm{O}^{\circ} \mathrm{C}$ to rt; (d) Lawesson's reagent, toluene, reflux.

(a) Under an argon atmosphere, a solution of cyclic imines $1 \mathrm{e}(0.25 \mathrm{mmol}),\left[\mathrm{Rh}(\mathrm{COE})_{2} \mathrm{Cl}\right]_{2}(1.5$ mol\%, $2.7 \mathrm{mg}, 0.0075 \mathrm{mmol}$ of Rh), ligand $(S)-\mathbf{L 1 1}$ ( $3 \mathrm{~mol} \%, 4.3 \mathrm{mg}, 0.0075 \mathrm{mmol}$ ), and arylboronic acid $(0.5 \mathrm{mmol})$ in $1.0 \mathrm{~mL}$ of toluene was stirred at room temperature for $30 \mathrm{~min}$. To this mixture was added aqueous $\mathrm{K}_{3} \mathrm{PO}_{4}(100 \mu \mathrm{L}, 2.5 \mathrm{M})$. After being stirred at room temperature overnight, the mixture was concentrated under reduced pressure. The residue was purified by silica gel column chromatography to afford the corresponding addition product $3 \mathbf{r}$ (98\% yield, 93\% ee).

3r: White solid, mp $137-138^{\circ} \mathrm{C}$; $[\alpha]_{\mathrm{D}}{ }^{25}=-25.2\left(c \mathrm{c} 0.52, \mathrm{CHCl}_{3}\right) ;{ }^{1} \mathrm{H}$ NMR $\left(300 \mathrm{MHz}, \mathrm{CDCl}_{3}\right) \delta$ $7.70(\mathrm{~s}, 1 \mathrm{H}), 7.51(\mathrm{t}, J=8.4 \mathrm{~Hz}, 2 \mathrm{H}), 7.44-7.32(\mathrm{~m}, 5 \mathrm{H}), 7.26(\mathrm{t}, J=8.0 \mathrm{~Hz}, 1 \mathrm{H}), 5.21(\mathrm{~s}, 1 \mathrm{H})$, $3.19(\mathrm{~s}, 3 \mathrm{H}) ;{ }^{13} \mathrm{C}$ NMR $\left(125 \mathrm{MHz}, \mathrm{CDCl}_{3}\right) \delta 168.1,139.9,138.2,132.5,130.7,130.4,129.7,129.3$, 127.6, 126.4, 123.1, 74.9, 26.5; IR (KBr) v 3146, 3113, 1707, 1415, 1342, 1220, 1185, 1067, 972 $\mathrm{cm}^{-1}$; HRMS (ESI) for $\mathrm{C}_{15} \mathrm{H}_{13} \mathrm{O}_{3} \mathrm{~N}_{2} \mathrm{BrSNa}[\mathrm{M}+\mathrm{Na}]^{+}$calcd 402.9722, found 402.9729 .

HPLC for compound 3r (its N-Boc derivative): Chiralpak AD-H column (250 mm); detected at $220 \mathrm{~nm}$; hexane $/ i$-propanol $=99 / 1$; flow $=1.0 \mathrm{~mL} / \mathrm{min}$; Retention time: $25.4 \mathrm{~min}$ (major), 27.9 $\min$.

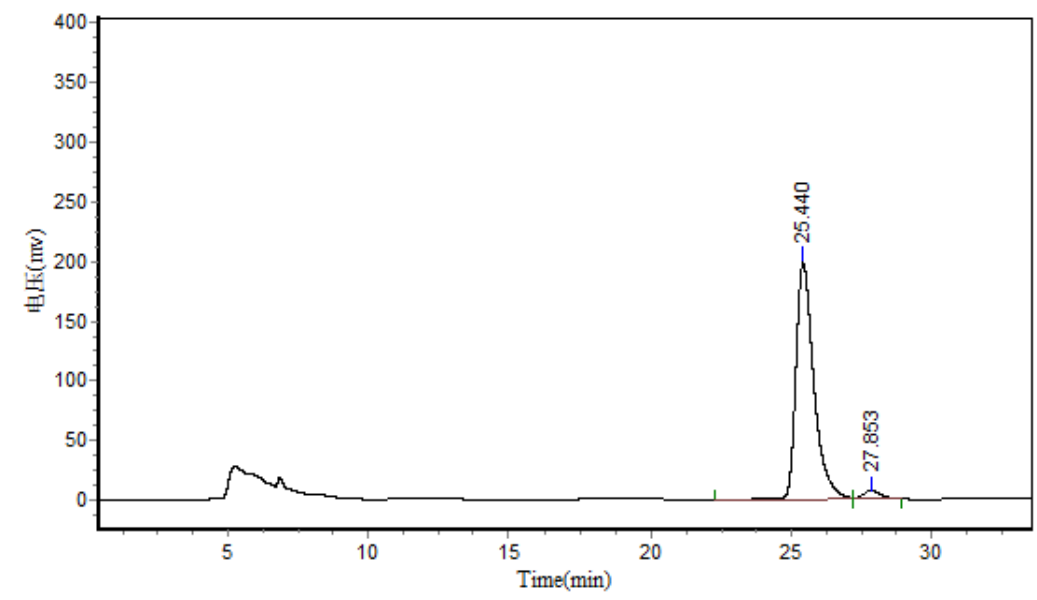

Results

\begin{tabular}{|c|c|c|c|c|c|}
\hline Peak No. & Peak ID & Ret Time & Height & Area & Conc. \\
\hline 1 & & 25.440 & 197846.266 & 8765014.000 & 96.2728 \\
\hline 2 & & 27.853 & 7414.521 & 339340.469 & 3.7272 \\
\hline Total & & & 205260.787 & 9104354.469 & 100.000 \\
\hline
\end{tabular}




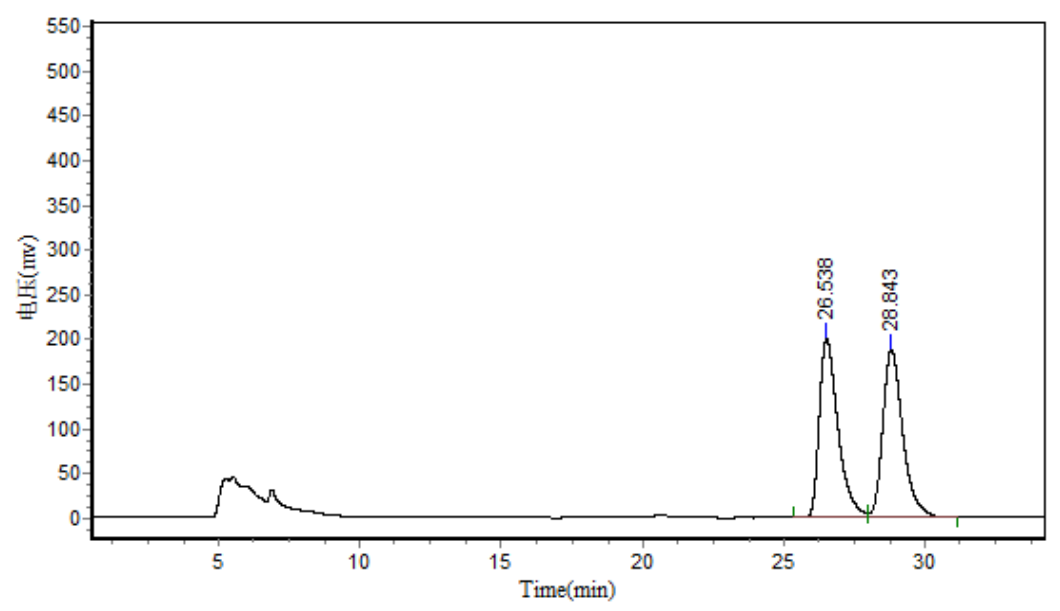

\begin{tabular}{|c|c|c|c|c|c|}
\hline \multicolumn{6}{|c|}{ Results } \\
\hline Peak No. & Peak ID & Ret Time & Height & Area & Conc. \\
\hline 1 & & 26.538 & 199183.109 & 9242049.000 & 49.7578 \\
\hline 2 & & 28.843 & 188069.719 & 9332036.000 & 50.2422 \\
\hline Total & & & 387252.828 & 18574085.000 & 100.0000 \\
\hline
\end{tabular}

(b) Compound $\mathbf{1 1}$ was synthesized according to the synthetic procedure of compound $\mathbf{6}$.

11: $86 \%$ yield, colorless oil, $[\alpha]_{\mathrm{D}}{ }^{25}=-4.4\left(c 0.435, \mathrm{CHCl}_{3}\right) ;{ }^{1} \mathrm{H} \mathrm{NMR}\left(300 \mathrm{MHz}, \mathrm{CDCl}_{3}\right) \delta 7.56(\mathrm{~s}$, $1 \mathrm{H}), 7.42(\mathrm{~d}, J=7.7 \mathrm{~Hz}, 1 \mathrm{H}), 7.38-7.27(\mathrm{~m}, 6 \mathrm{H}), 7.19(\mathrm{t}, J=7.8 \mathrm{~Hz}, 1 \mathrm{H}), 2.85(\mathrm{~d}, J=4.9 \mathrm{~Hz}$, $3 \mathrm{H}), 2.04(\mathrm{~s}, 3 \mathrm{H}) ;{ }^{13} \mathrm{C} \mathrm{NMR}\left(125 \mathrm{MHz}, \mathrm{CDCl}_{3}\right) \delta 173.7,147.6,144.5,130.7,130.6,130.0,128.6$, 127.8, 127.5, 126.7, 122.6, 67.9, 26.7; IR (KBr) v 3365, 3309, 1663, 1591, 1518, 1470, 1446, $1408,1158,1074 \mathrm{~cm}^{-1}$; HRMS (ESI) for $\mathrm{C}_{15} \mathrm{H}_{16} \mathrm{ON}_{2} \mathrm{Br}[\mathrm{M}+\mathrm{H}]^{+}$calcd 319.0441, found 319.0436.

(c) Compound 12 was synthesized according to the synthetic procedure of compound $\mathbf{9}$.

12: $71 \%$ yield, colorless oil; $[\alpha]_{\mathrm{D}}{ }^{25}=-27.7\left(c 0.68, \mathrm{CHCl}_{3}\right) ;{ }^{1} \mathrm{H}$ NMR $\left(300 \mathrm{MHz}, \mathrm{CDCl}_{3}\right) \delta 7.57(\mathrm{~d}$, $J=1.4 \mathrm{~Hz}, 1 \mathrm{H}), 7.49(\mathrm{~d}, J=7.8 \mathrm{~Hz}, 1 \mathrm{H}), 7.44-7.30(\mathrm{~m}, 6 \mathrm{H}), 7.23(\mathrm{~d}, J=7.8 \mathrm{~Hz}, 1 \mathrm{H}), 6.70(\mathrm{~s}$, $1 \mathrm{H}), 3.10(\mathrm{~s}, 3 \mathrm{H}) ;{ }^{13} \mathrm{C} \mathrm{NMR}\left(125 \mathrm{MHz}, \mathrm{CDCl}_{3}\right) \delta 173.0,156.7,141.3,138.7,132.0,130.5,130.0$, 129.2, 129.0, 126.8, 125.9, 123.1, 69.9, 25.3; IR (KBr) v 3285, 2961, 2925, 2853, 1772, 1702, 1459, 1448, 1394, 1262, $1074 \mathrm{~cm}^{-1}$; HRMS (ESI) for $\mathrm{C}_{16} \mathrm{H}_{14} \mathrm{O}_{2} \mathrm{~N}_{2} \mathrm{Br}[\mathrm{M}+\mathrm{H}]^{+}$calcd 345.0233, found 345.0229 .

(d) A solution of compound 12 (69 mg, $0.2 \mathrm{mmol}, 1$ equiv) and Lawesseon's reagent (2 equiv) in $5 \mathrm{~mL}$ toluene was stirred at $100{ }^{\circ} \mathrm{C}$ overnight. The mixture was concentrated on a rotary evaporator and the residue was purified by silica gel column chromatography to afford the corresponding addition product $\mathbf{1 3}$ (68 $\mathrm{mg}, 95 \%$ yield, $93 \%$ ee).

13: Colorless oil, $[\alpha]_{\mathrm{D}}{ }^{25}=-38.8\left(c 0.275, \mathrm{CHCl}_{3}\right) ;{ }^{1} \mathrm{H} \mathrm{NMR}\left(300 \mathrm{MHz}, \mathrm{CDCl}_{3}\right) \delta 8.25(\mathrm{~s}, 1 \mathrm{H})$, $7.59-7.45$ (m, 2H), $7.42-7.34$ (M, 3H), $7.33-7.20$ (m, 4H), $3.33(\mathrm{~s}, 3 \mathrm{H}) ;{ }^{13} \mathrm{C}$ NMR (125 MHz, $\left.\mathrm{CDCl}_{3}\right) \delta 183.0,173.1,140.0,137.5,132.3,130.6,130.0,129.4,126.9,125.8,123.2,71.9,28.3$; IR (KBr) $v 3447,2954,2924,2854,1747,1729,1507,1467,1447,1294,1144 \mathrm{~cm}^{-1}$; HRMS (ESI) for $\mathrm{C}_{16} \mathrm{H}_{14} \mathrm{ON}_{2} \mathrm{BrS}[\mathrm{M}+\mathrm{H}]^{+}$calcd 361.0005 found 360.9999 .

HPLC for compound 13: Chiral AD-H column $(250 \mathrm{~mm})$; detected at $220 \mathrm{~nm}$; hexane/i-propanol $=95 / 5 ;$ flow $=0.7 \mathrm{~mL} / \mathrm{min}$; Retention time: $18.3 \mathrm{~min}$ (major), $22.2 \mathrm{~min}$ 


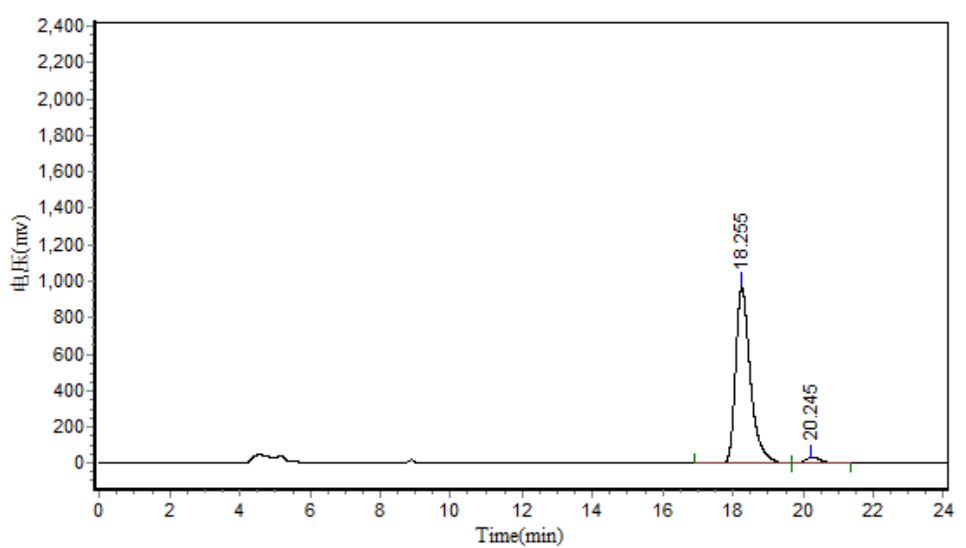

Results

\begin{tabular}{|c|c|c|c|c|c|}
\hline Peak No. & Peak ID & Ret Time & Height & Area & Conc. \\
\hline 1 & & 18.255 & 967965.500 & 28204196.000 & 96.2518 \\
\hline 2 & & 20.245 & 35190.918 & 1098303.500 & 3.7482 \\
\hline Total & & & 1003156.418 & 29302499.500 & 100.0000 \\
\hline
\end{tabular}

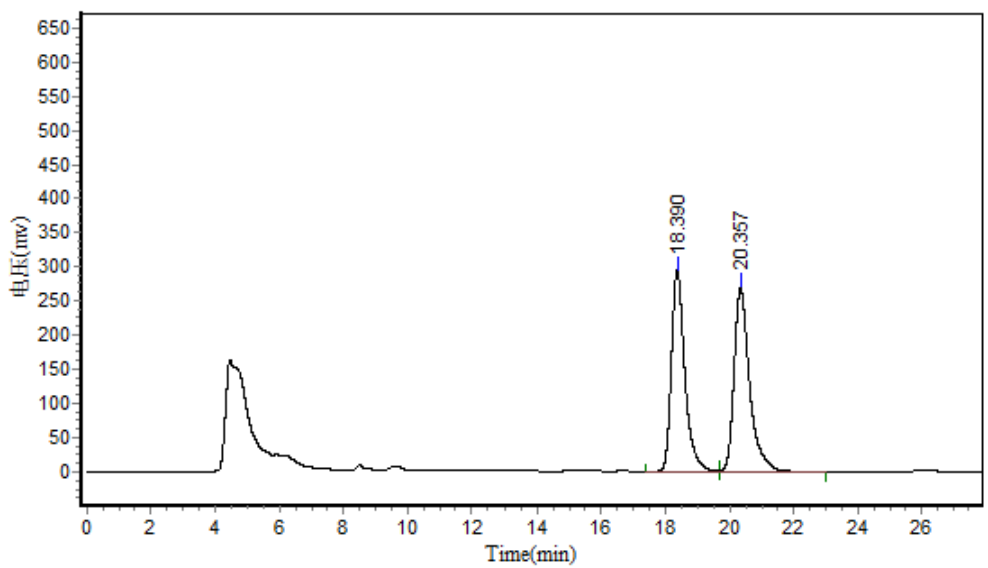

Results

\begin{tabular}{|c|c|c|c|c|c|}
\hline Peak No. & Peak ID & Ret Time & Height & Area & Conc. \\
\hline 1 & & 18.390 & 294386.500 & 8567858.000 & 48.7226 \\
\hline 2 & & 20.357 & 268262.938 & 9017104.000 & 51.2774 \\
\hline Total & & & 562649.438 & 17584962.000 & $100.000 \mathrm{c}$ \\
\hline
\end{tabular}


10. Copies of ${ }^{1} \mathrm{H}$ NMR and ${ }^{13} \mathrm{C}$ NMR spectra of compounds $3,5-13$

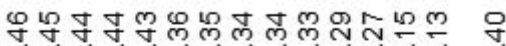

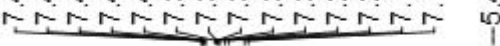

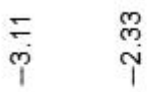

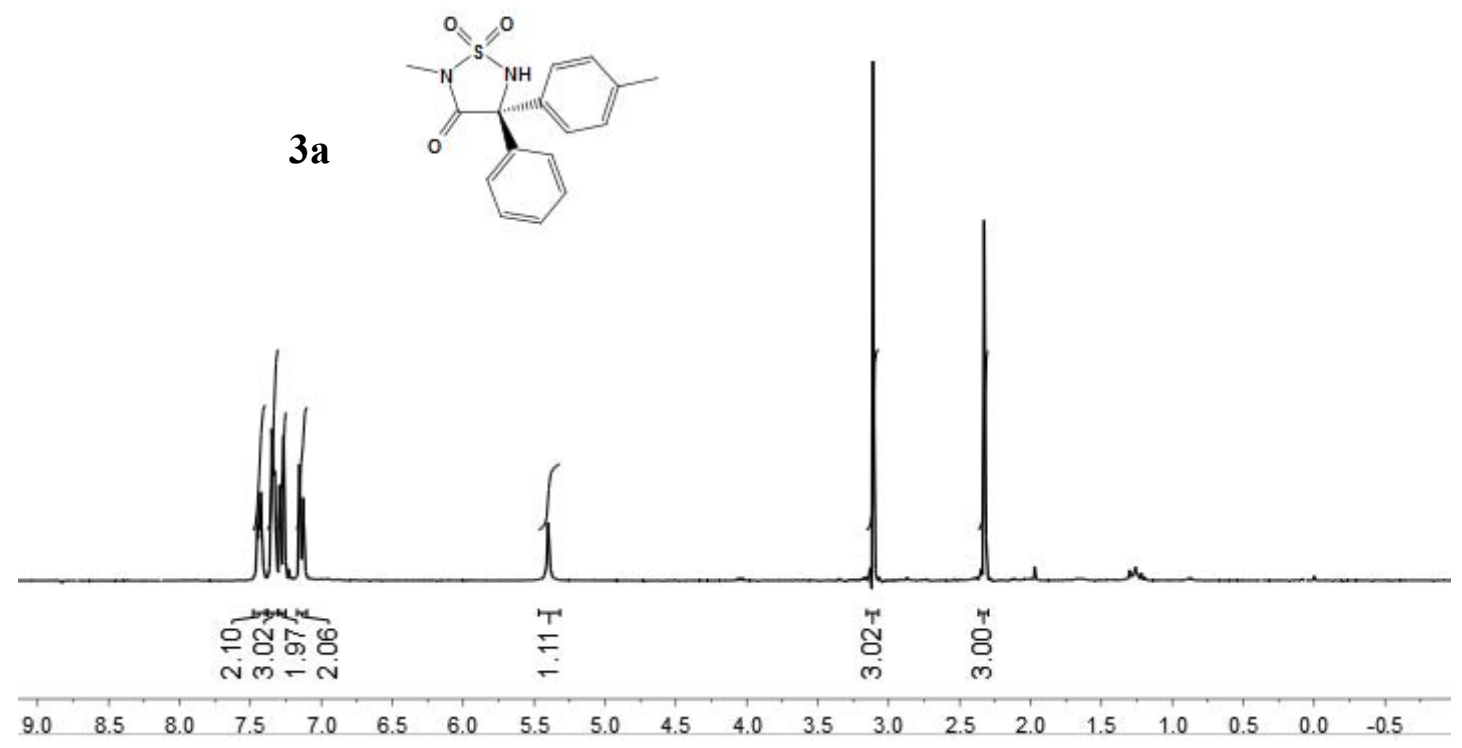

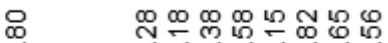

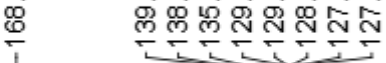

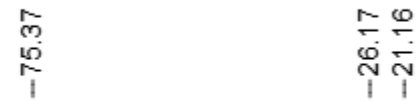

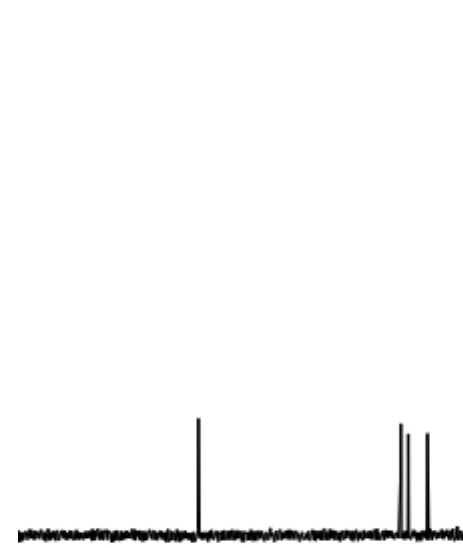

3a

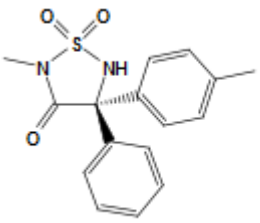

$\begin{array}{lllllllllllllllllllll}190 & 180 & 170 & 160 & 150 & 140 & 130 & 120 & 110 & 100 & 90 & 80 & 70 & 60 & 50 & 40 & 30 & 20 & 10 & 0 & -10\end{array}$ 
3b
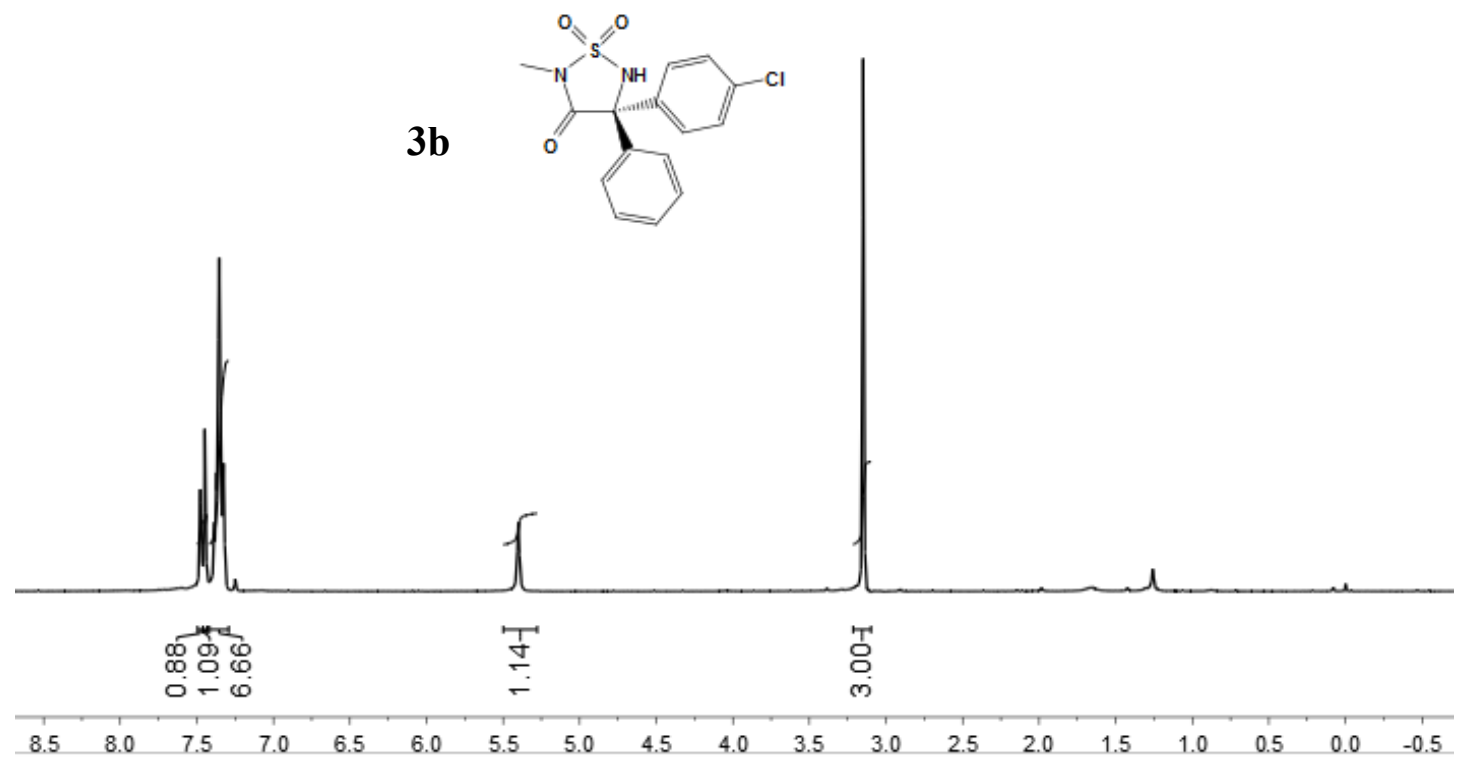

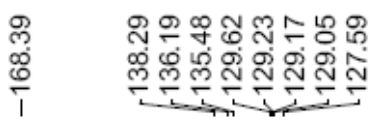

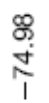

ले

3b<smiles>CN1C(=O)[C@](c2ccccc2)(c2ccc(Cl)cc2)NS1(=O)=O</smiles>

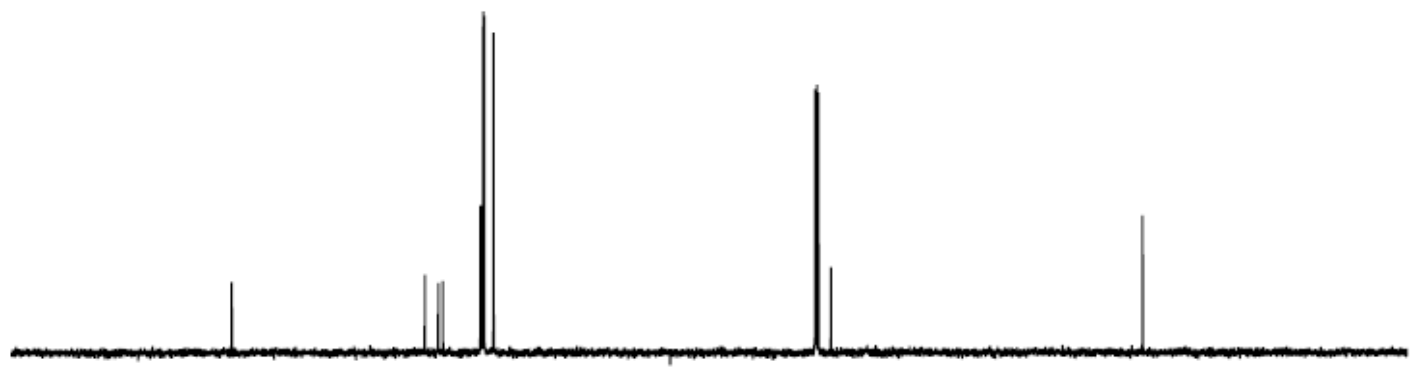

$\begin{array}{llllllllllllllllllllllll}1 & 1 \\ 200 & 190 & 180 & 170 & 160 & 150 & 140 & 130 & 120 & 110 & 100 & 90 & 80 & 70 & 60 & 50 & 40 & 30 & 20 & 10 & 0 & -10\end{array}$ 


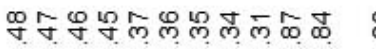

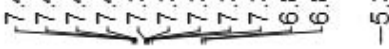

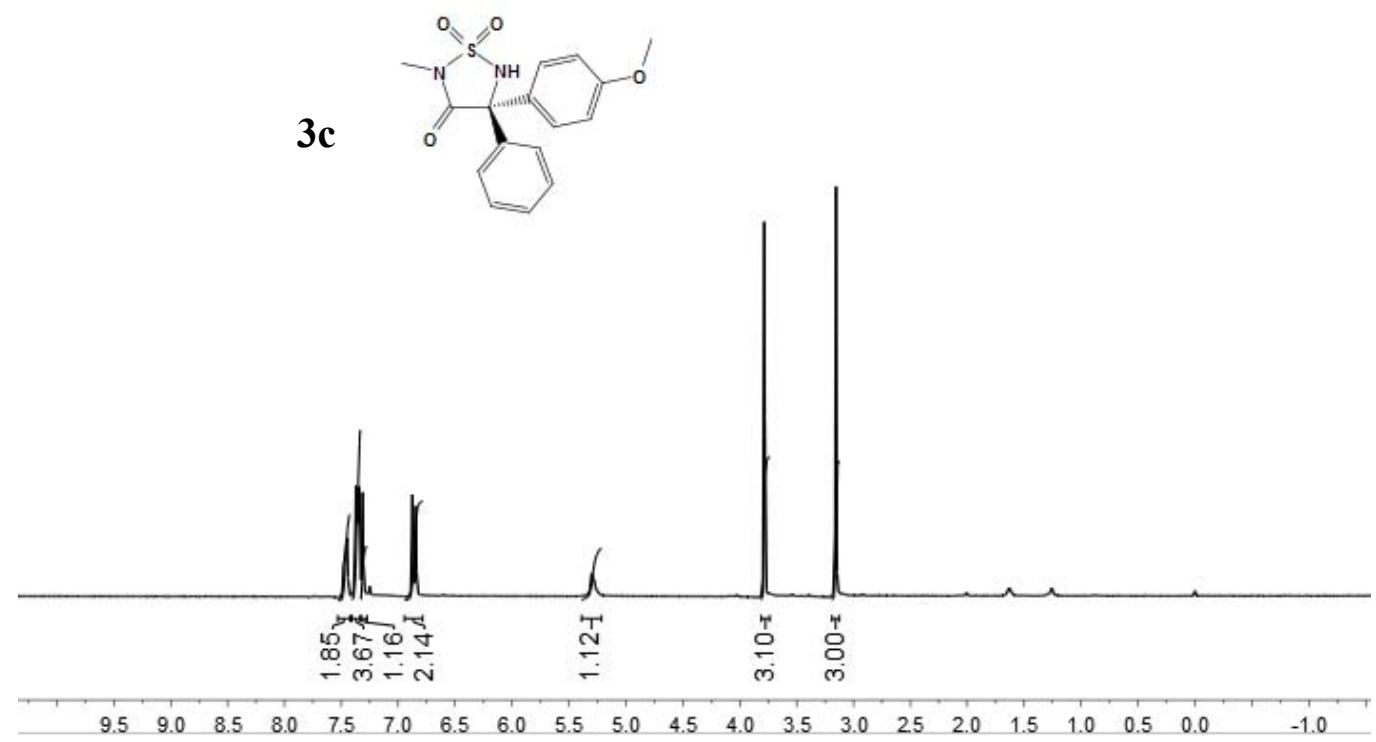

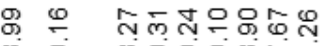

$\overbrace{}^{1}$

\begin{tabular}{lll}
0 & 0 & \multirow{2}{n}{} \\
0 & 0 & 0 \\
1 & 0 & 0
\end{tabular}
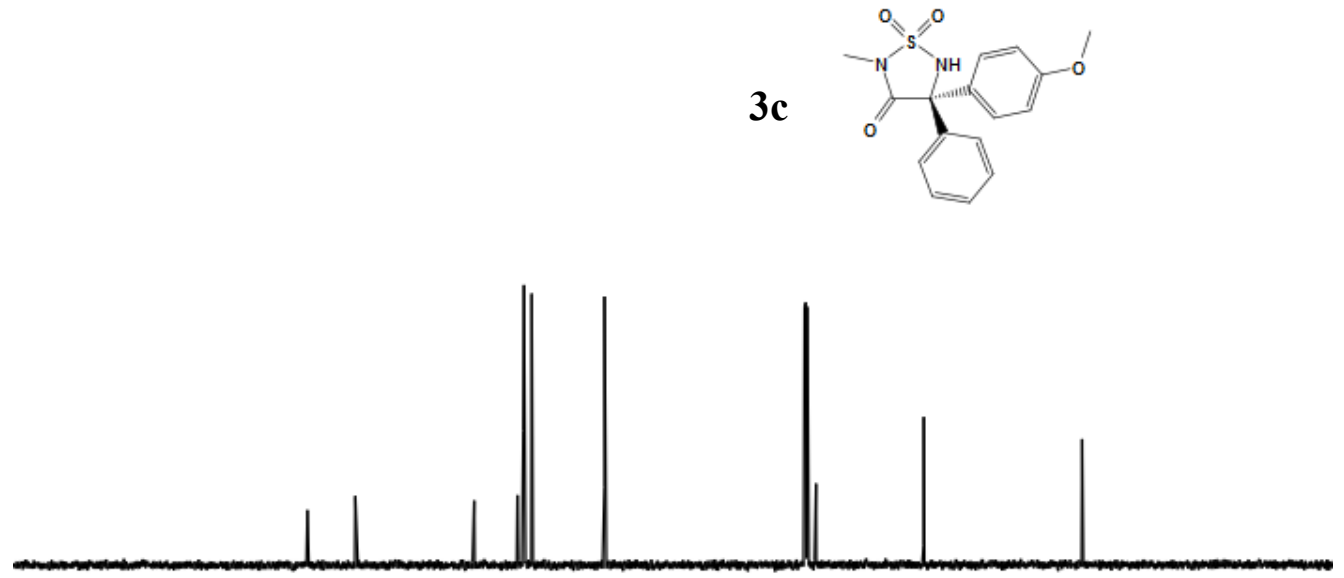

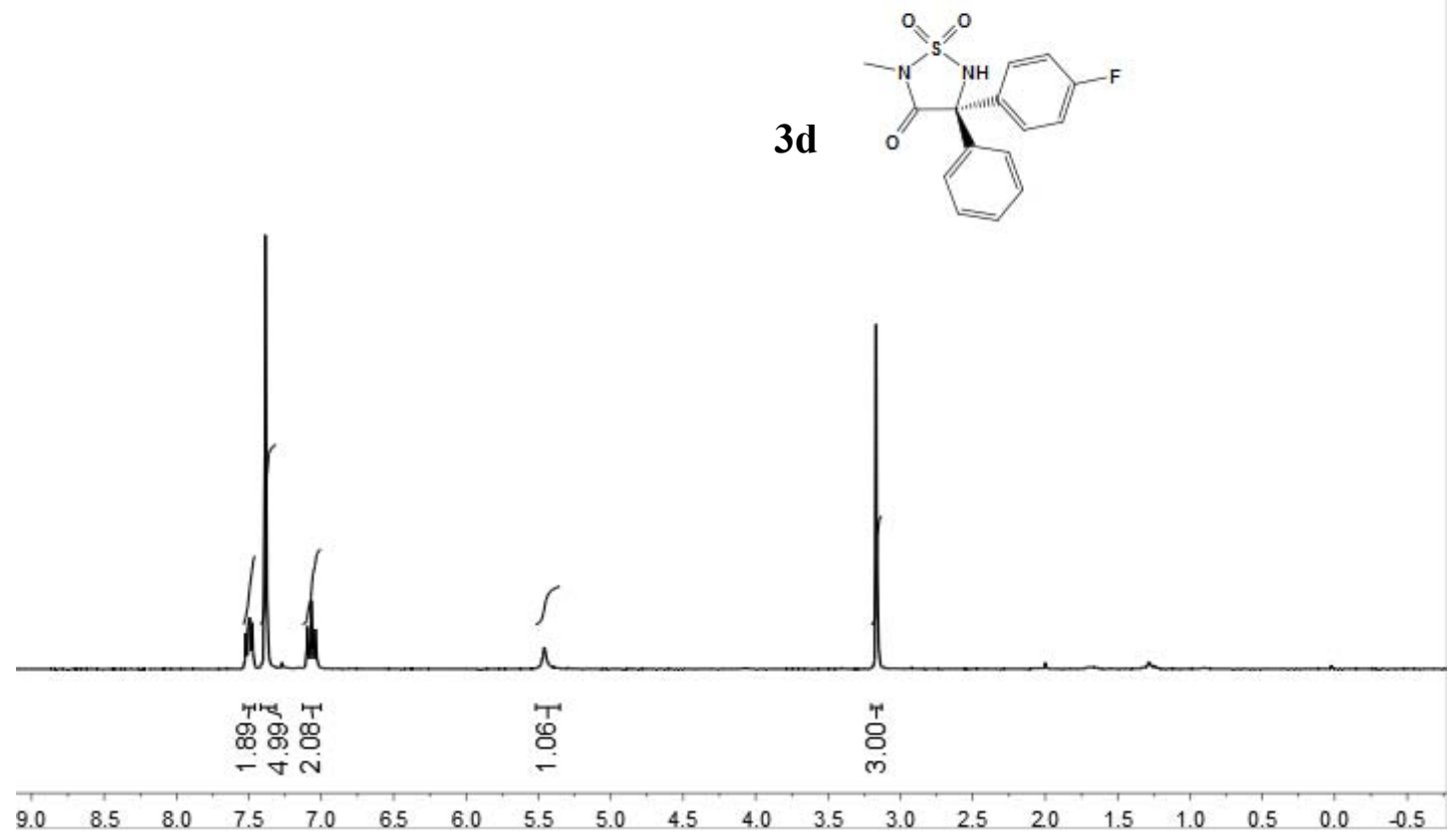

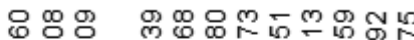

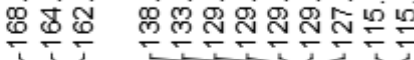

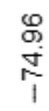

$\stackrel{\substack{n \\ i}}{i}$

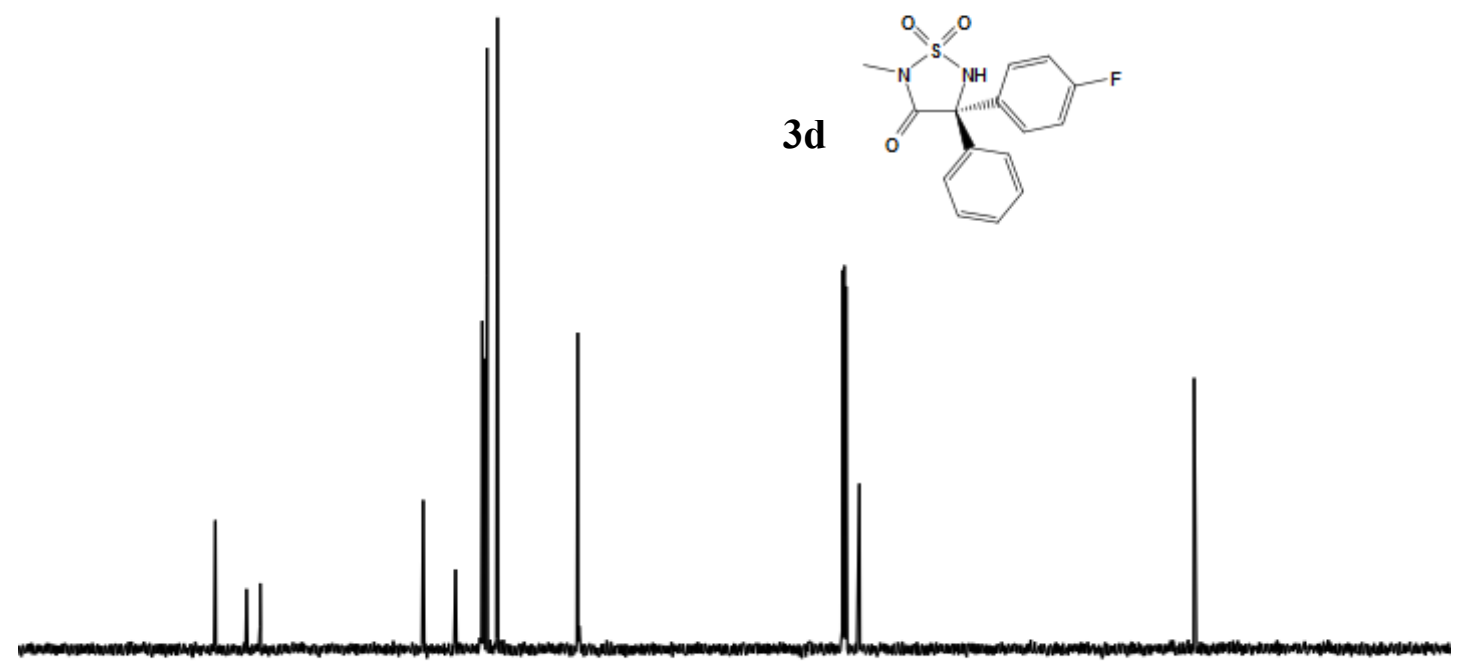

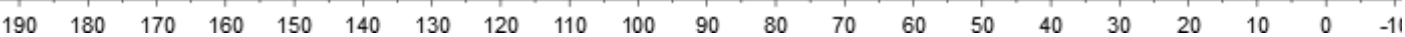



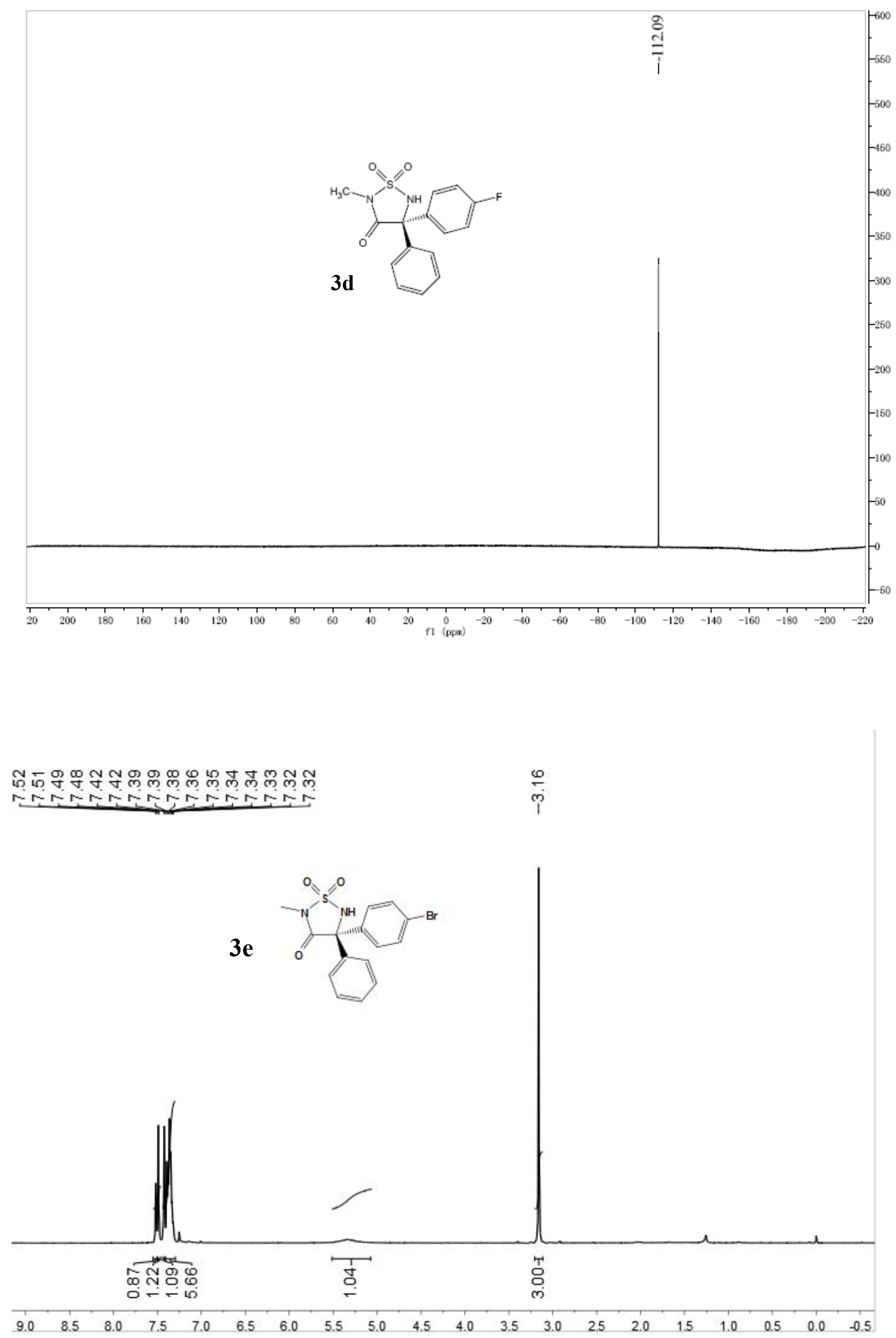


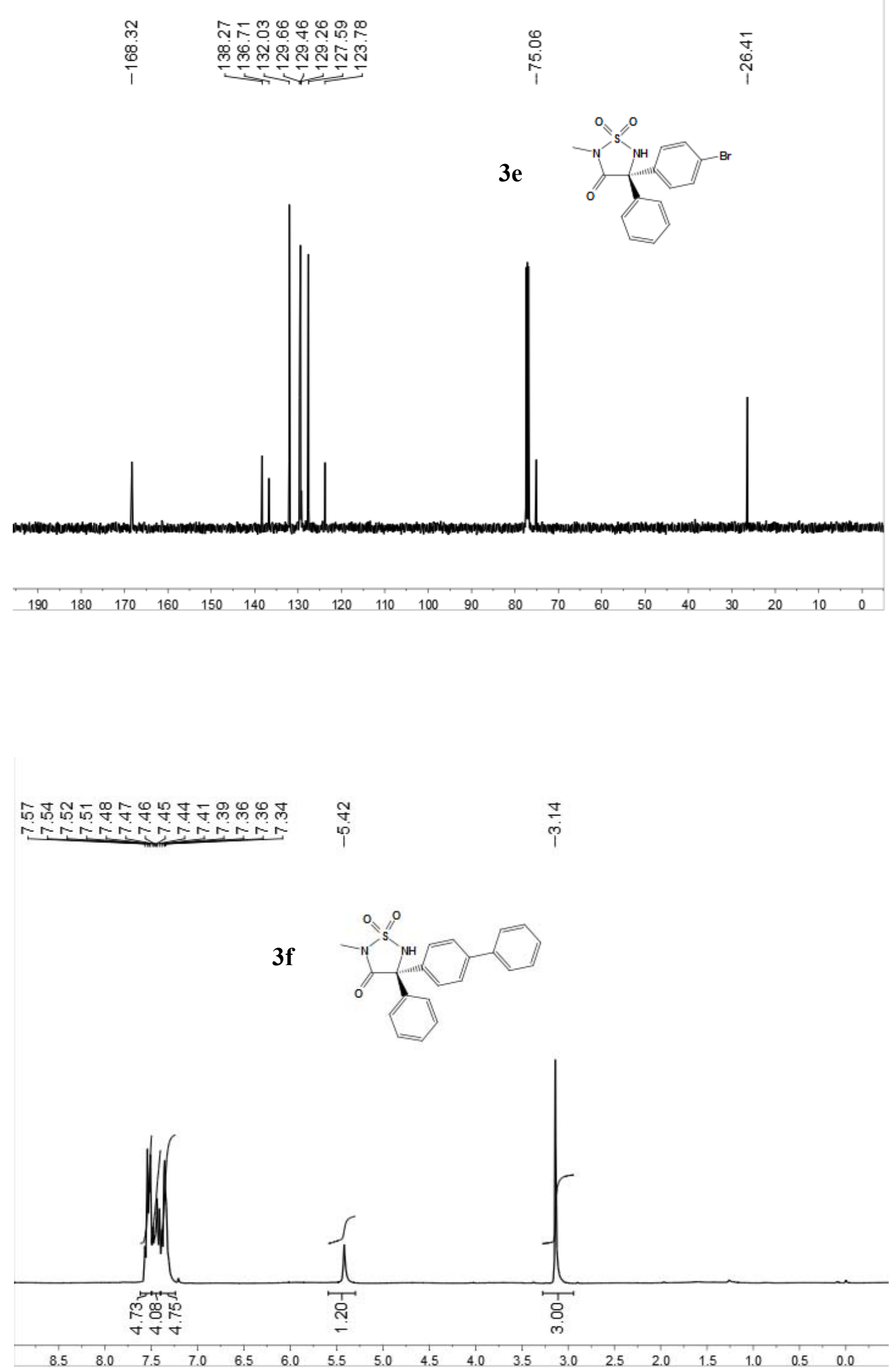


$\begin{array}{ll}\stackrel{\infty}{0} & \stackrel{\infty}{\infty} \\ \stackrel{0}{i} & \stackrel{1}{1}\end{array}$

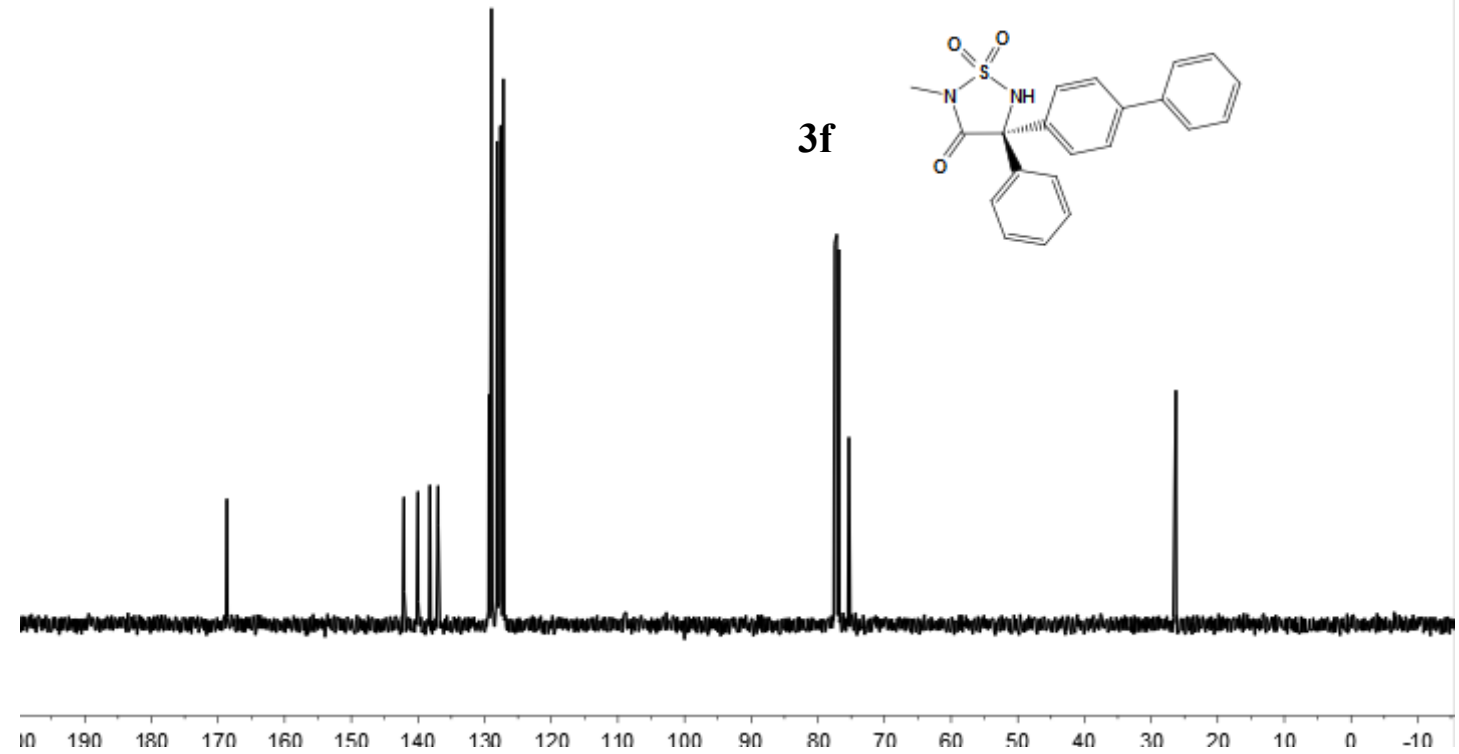

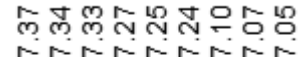

im

$\stackrel{p}{p}$

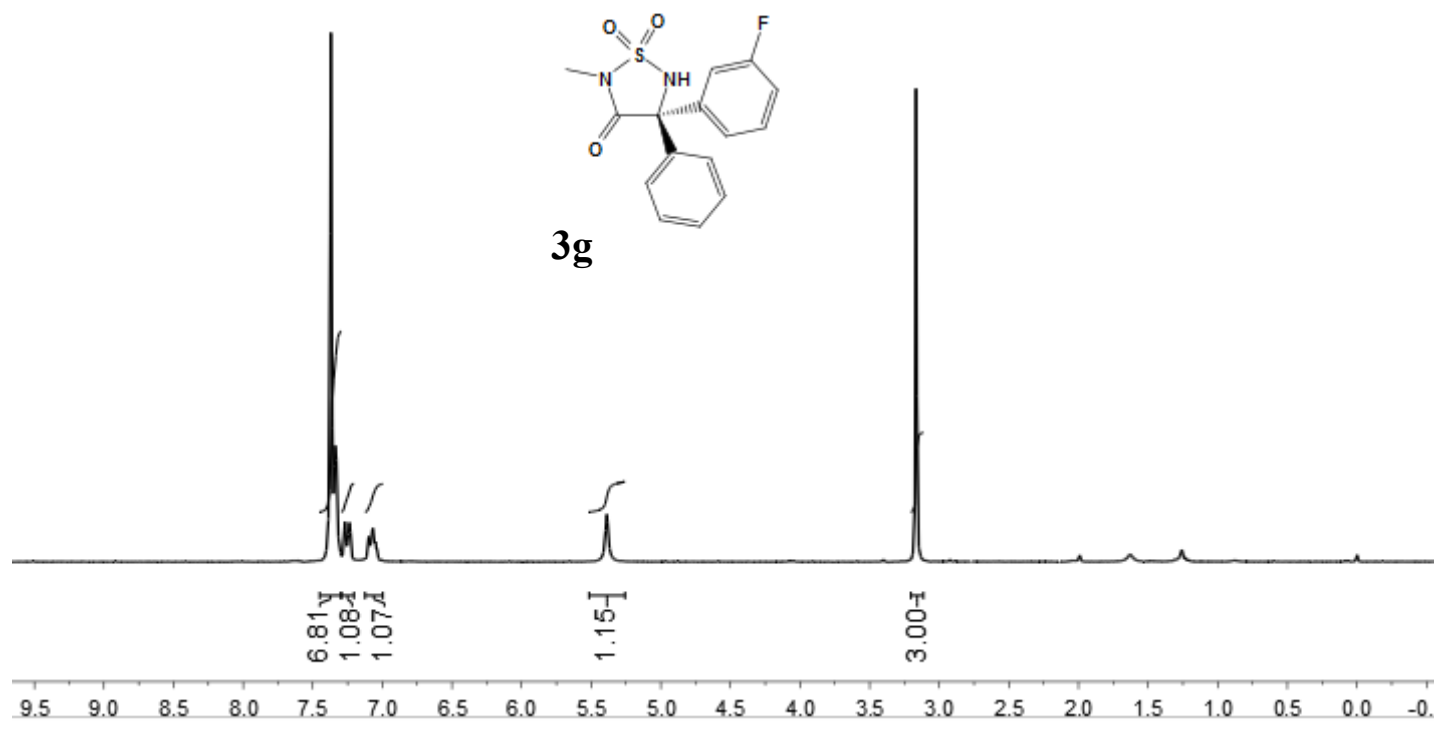




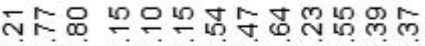

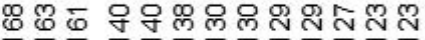

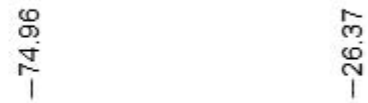

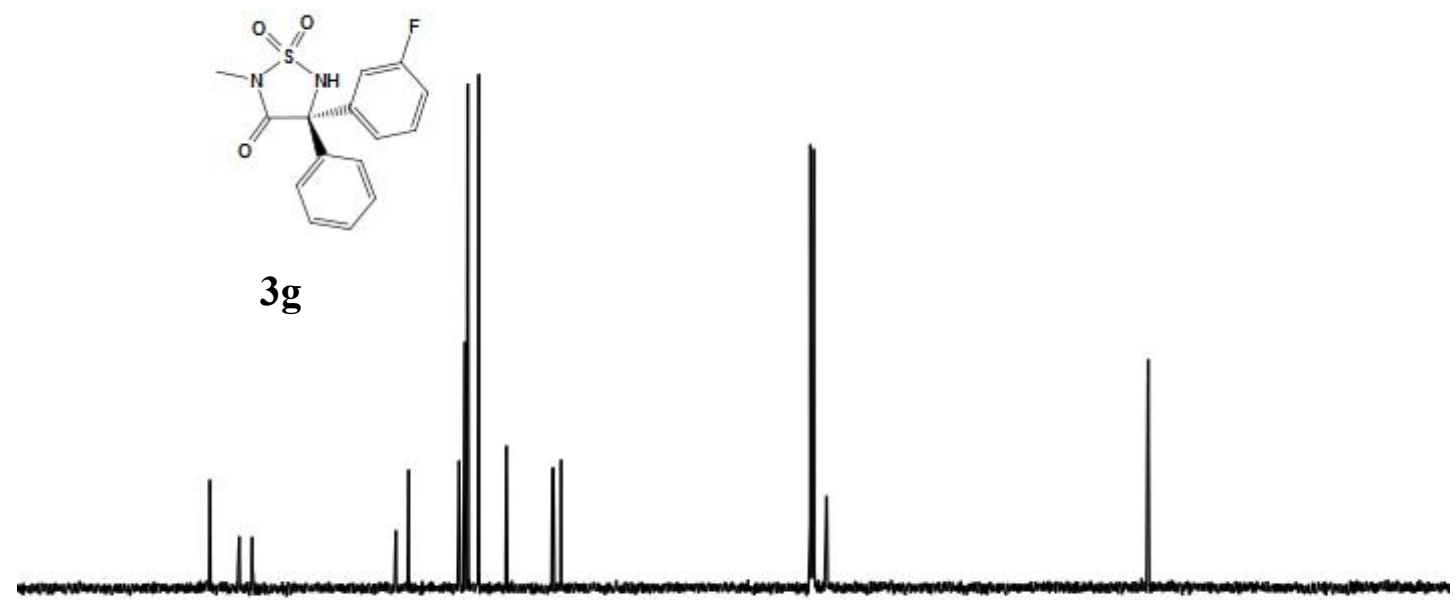

$\begin{array}{lllllllllllllllllllll}190 & 180 & 170 & 160 & 150 & 140 & 130 & 120 & 110 & 100 & 90 & 80 & 70 & 60 & 50 & 40 & 30 & 20 & 10 & 0 & -10\end{array}$

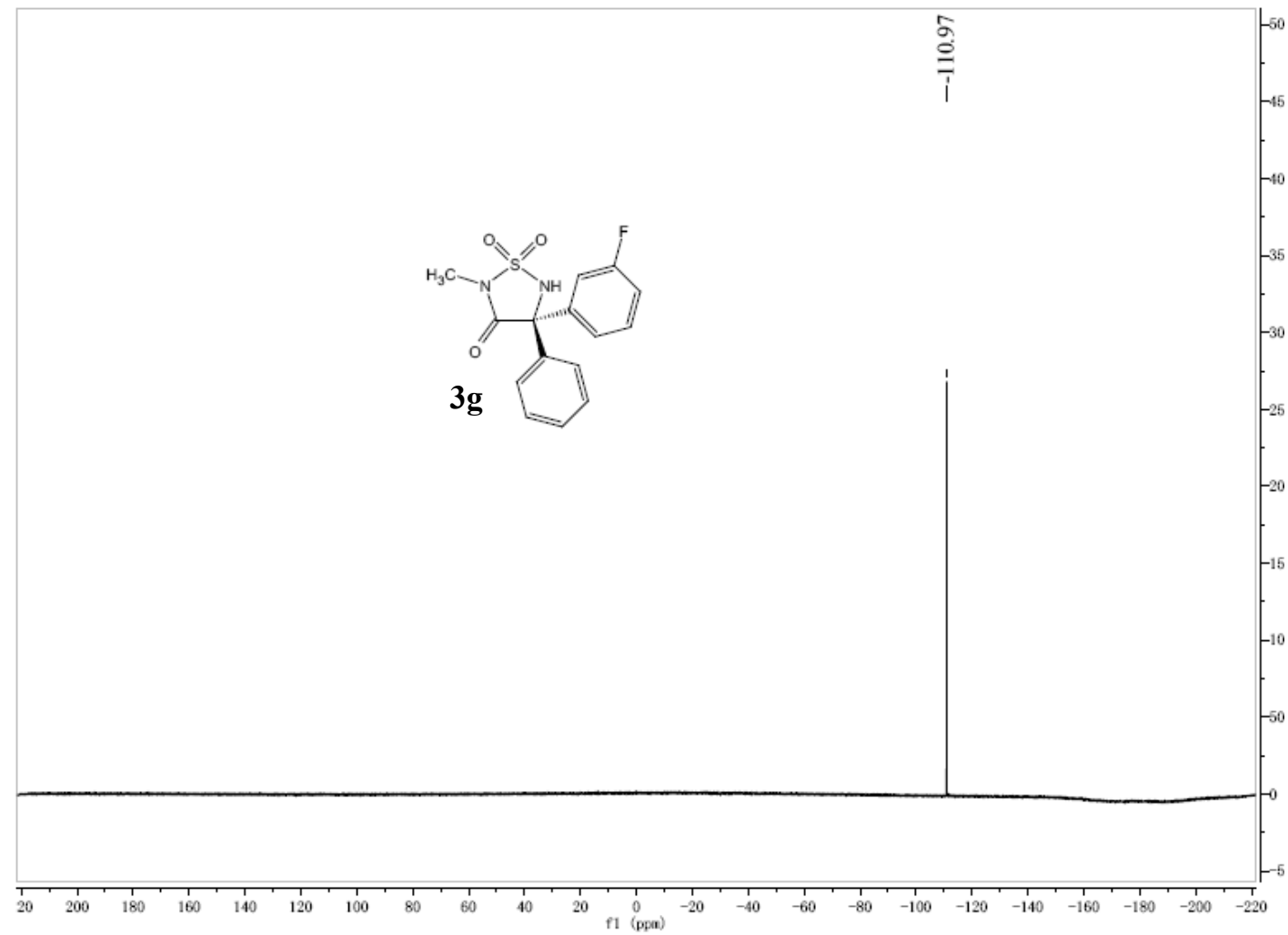




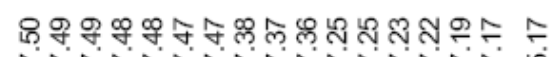

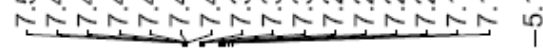

$\stackrel{p}{p} \quad \stackrel{m}{i}$

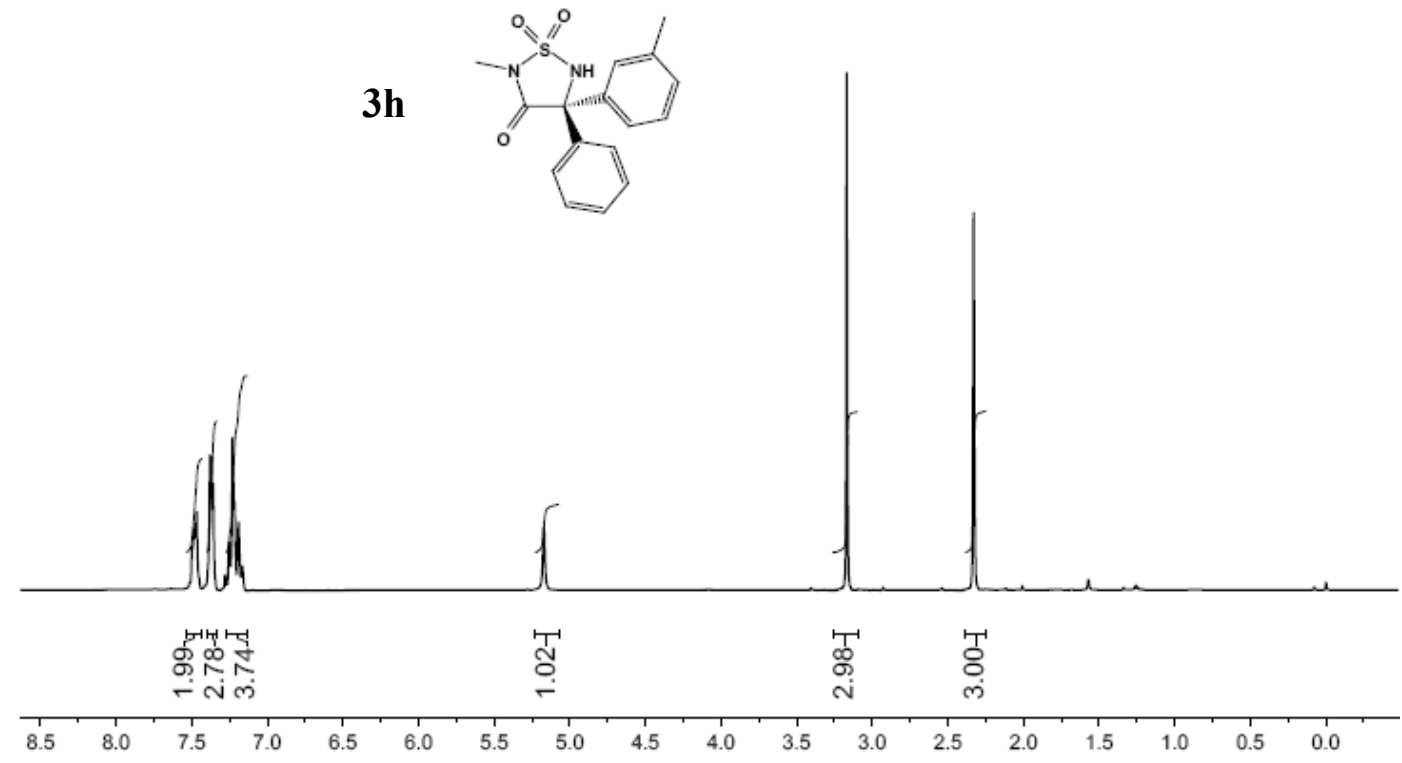

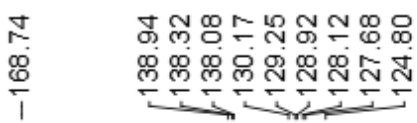

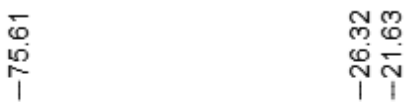

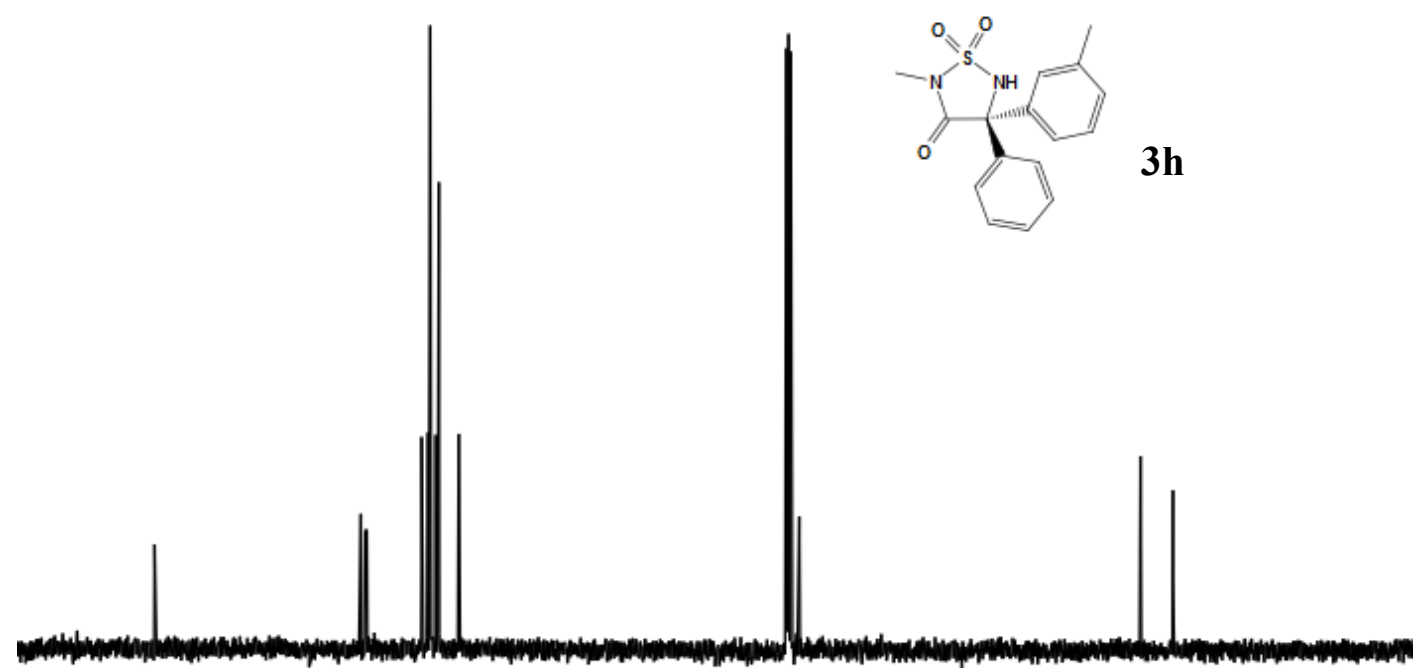

$\begin{array}{llllllllllllllllllll}180 & 170 & 160 & 150 & 140 & 130 & 120 & 110 & 100 & 90 & 80 & 70 & 60 & 50 & 40 & 30 & 20 & 10 & 0 & -10\end{array}$ 

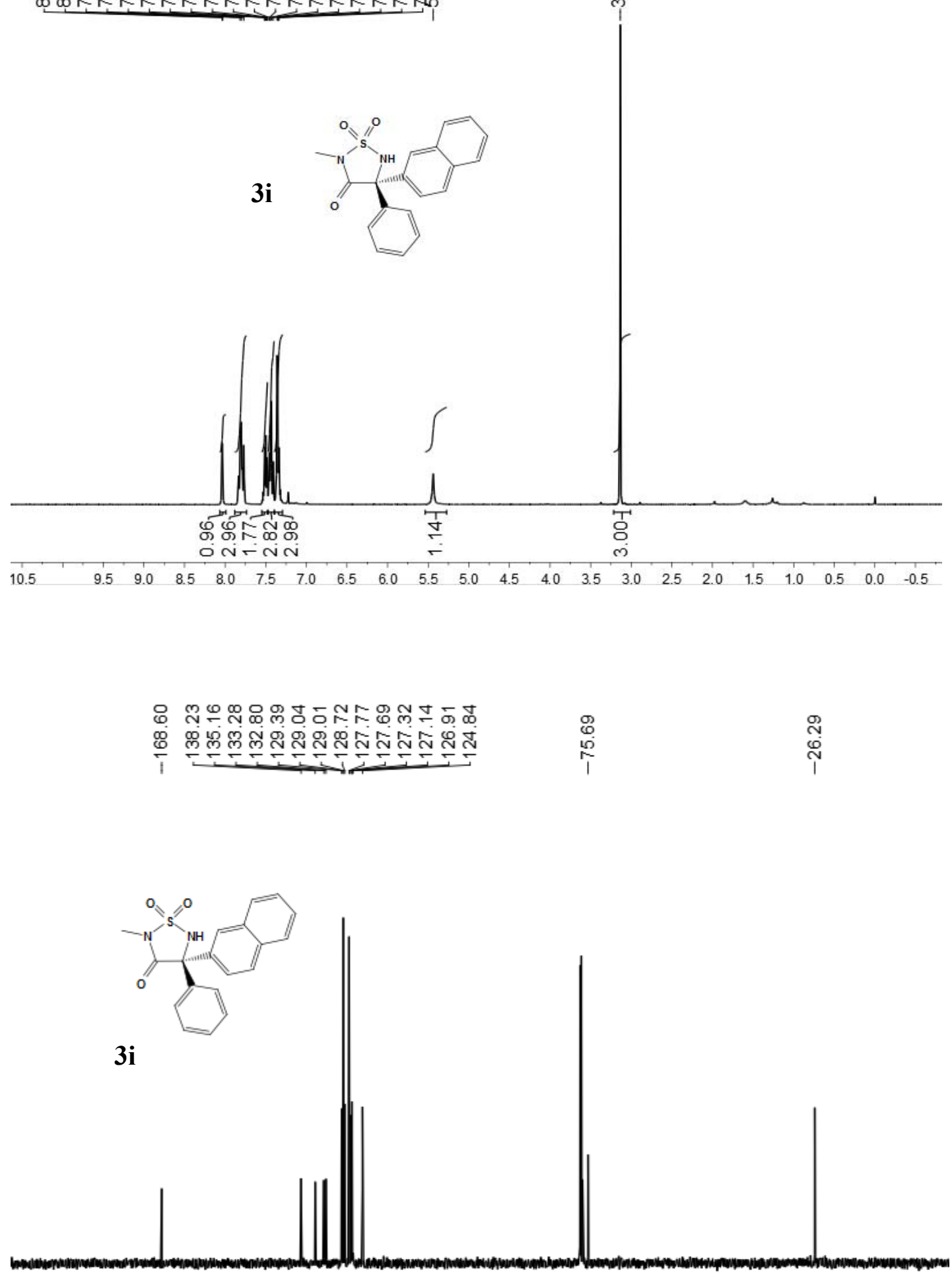

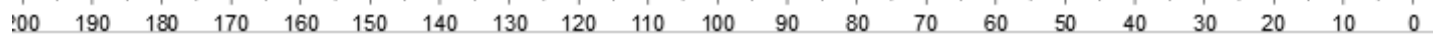




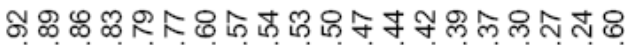

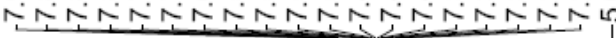
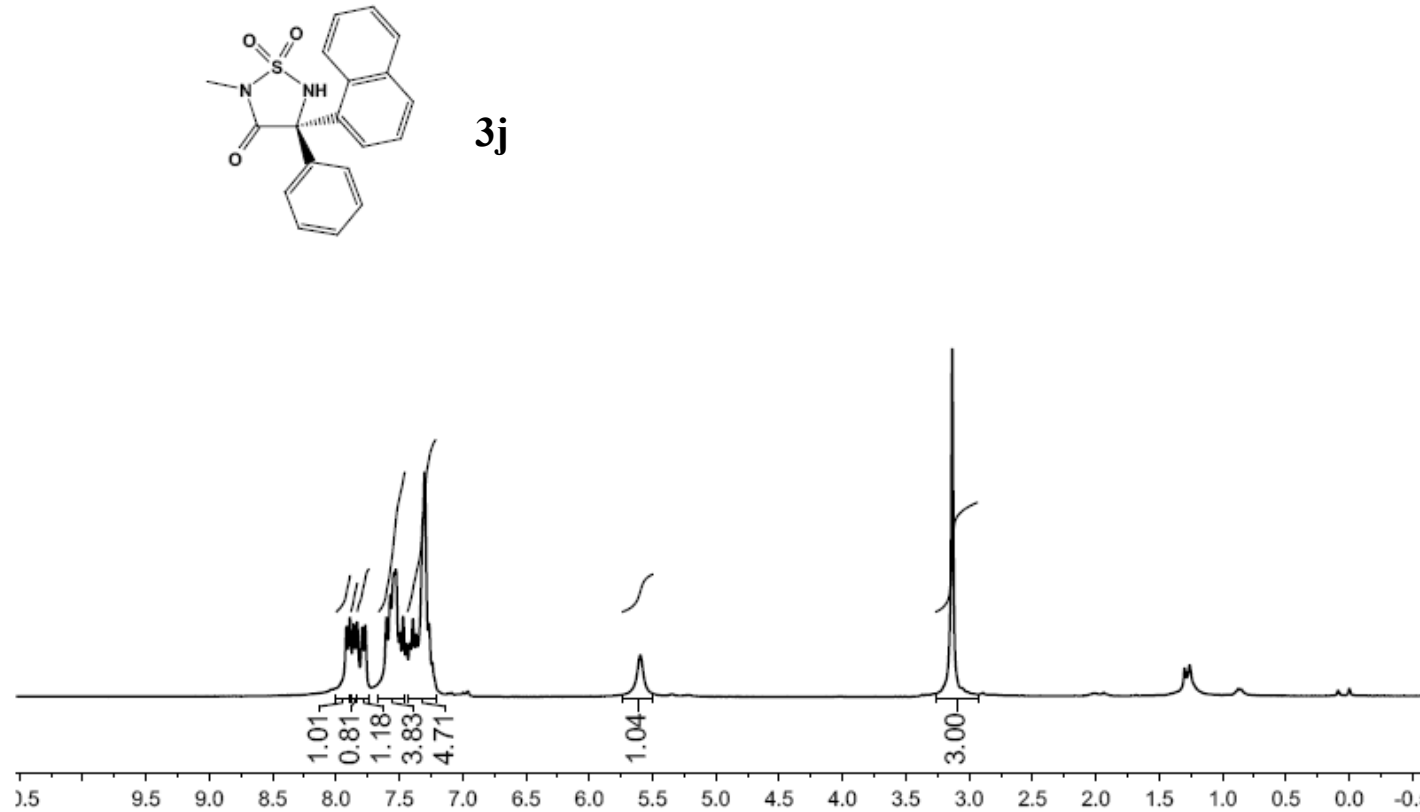

\& f

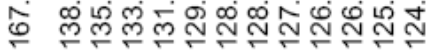

\begin{tabular}{ll}
0 & $\stackrel{2}{0}$ \\
0 & \multirow{2}{0}{}
\end{tabular}

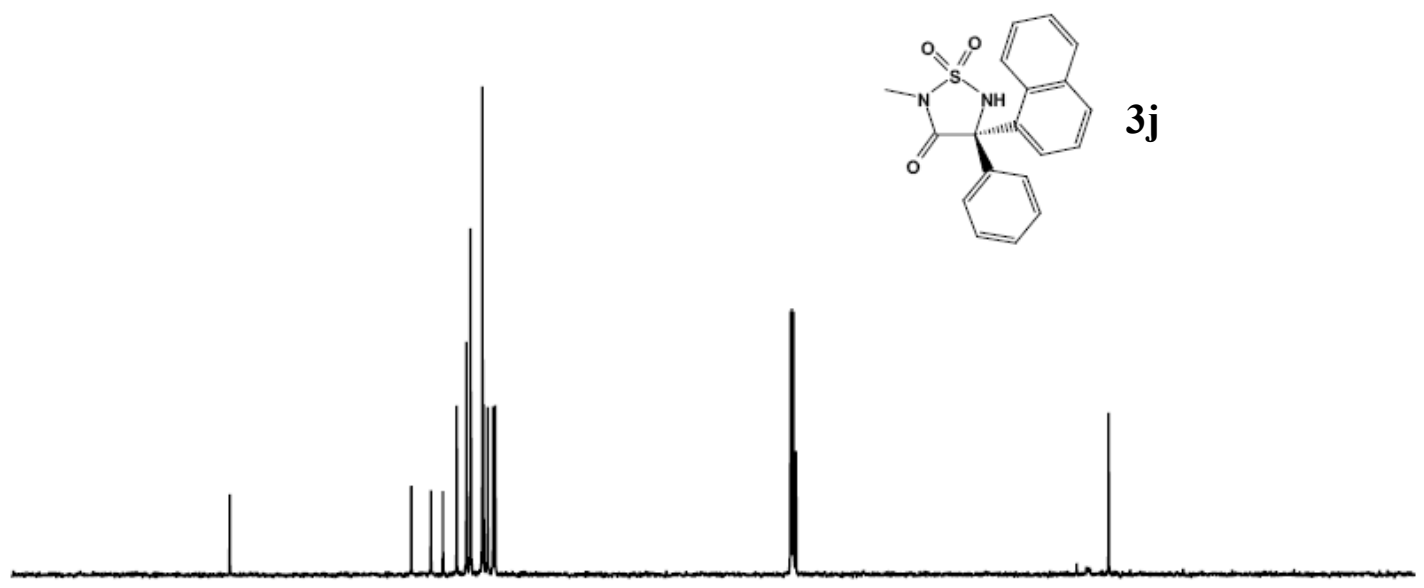

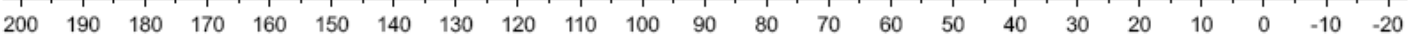


மீ

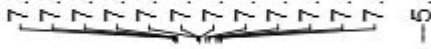

$\stackrel{\circ}{\stackrel{\circ}{p}}$

3k

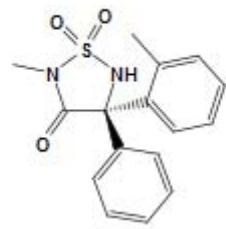

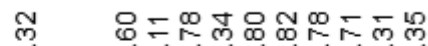

눈

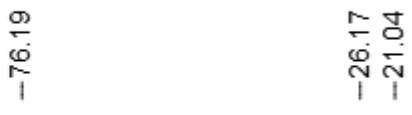

$3 \mathbf{k}$
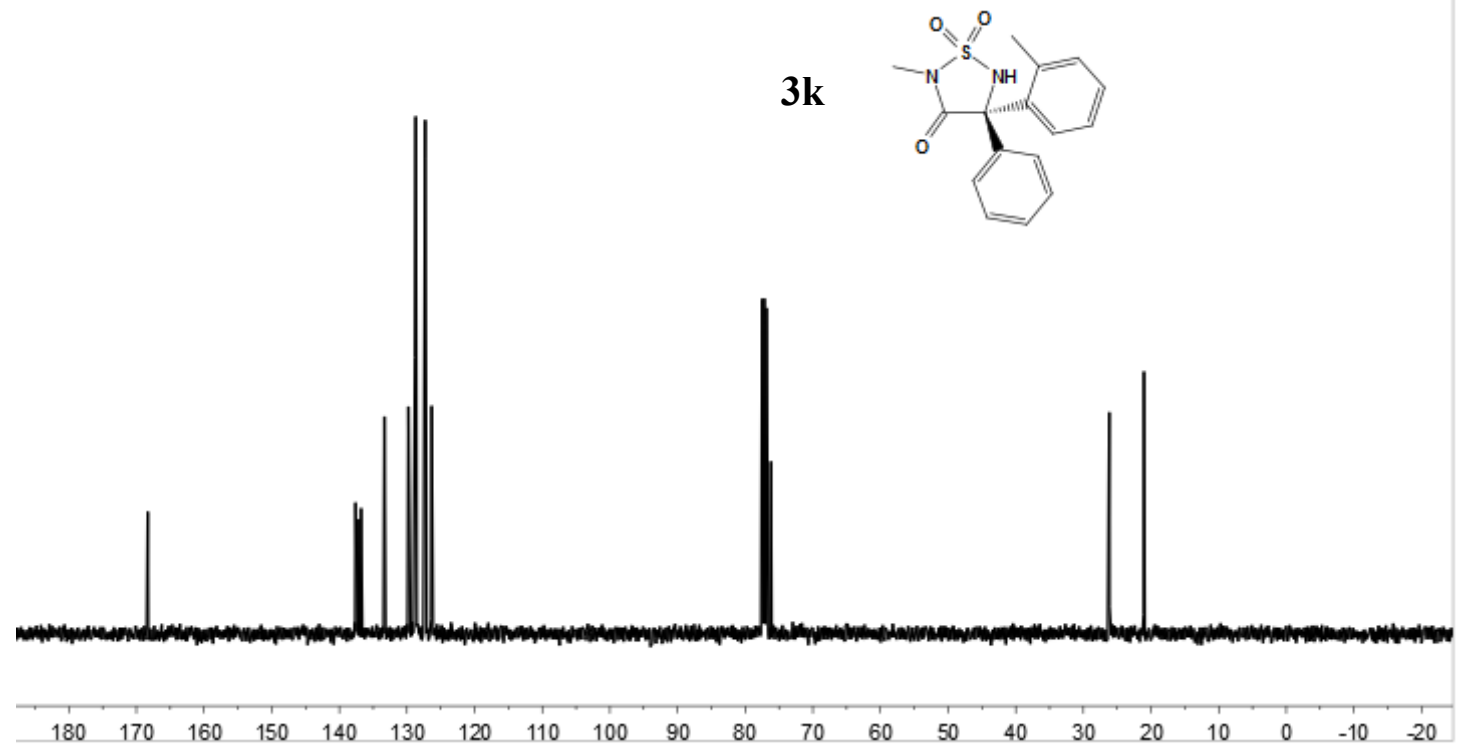

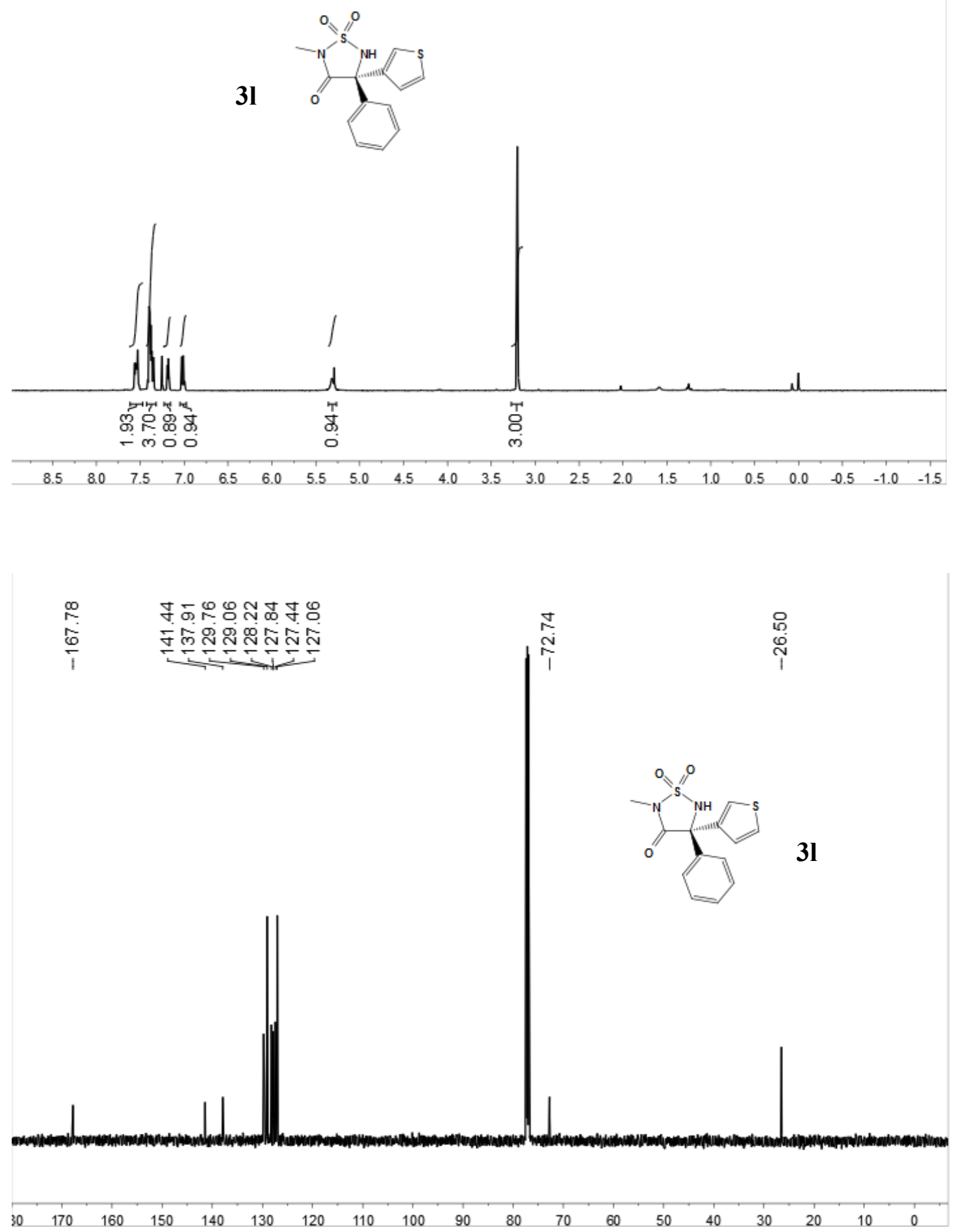


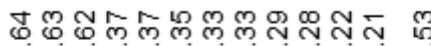

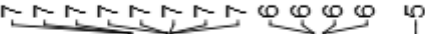

$3 m$
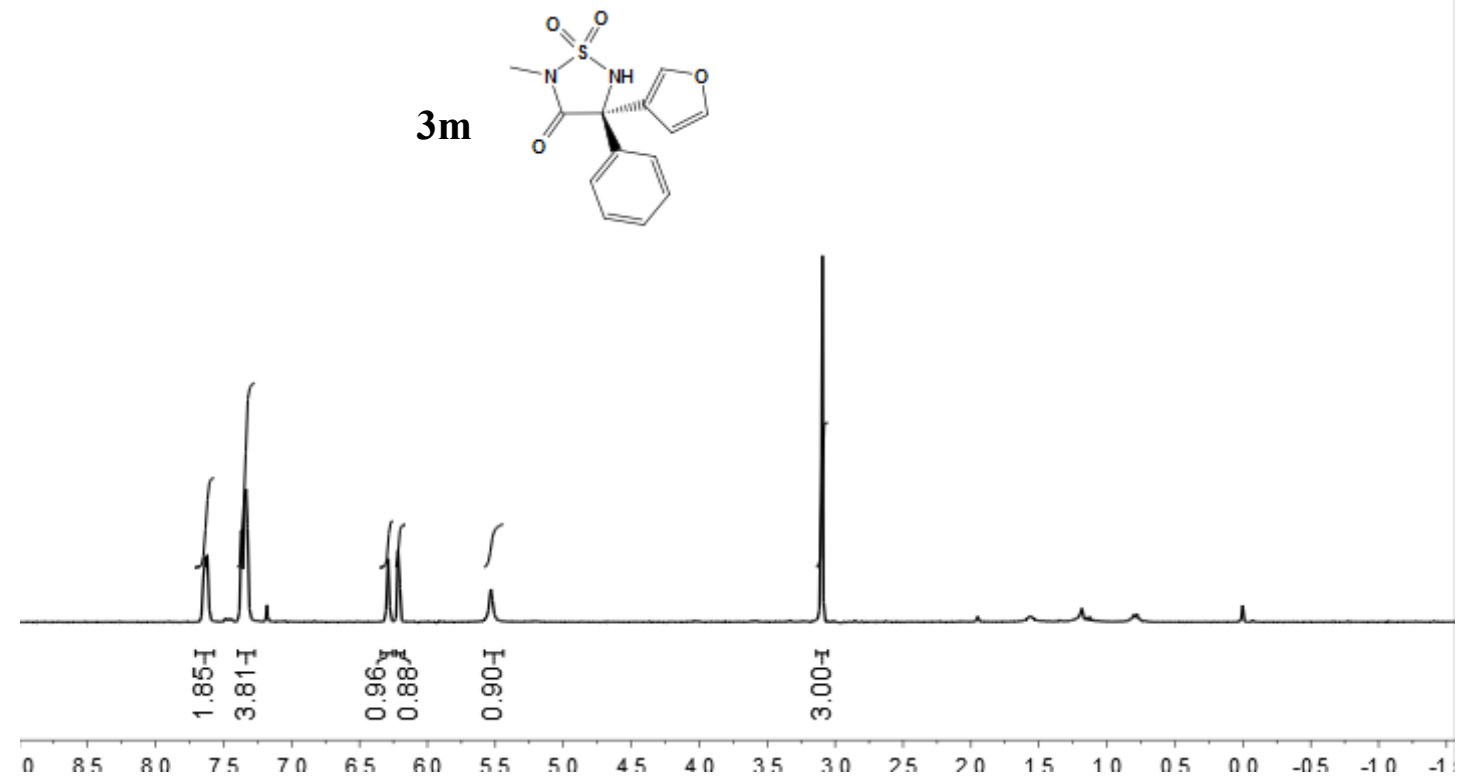

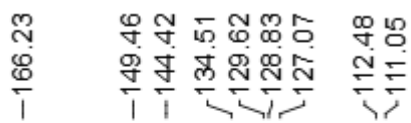

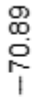

$\underset{\substack{\infty \\ ָ}}{\stackrel{\infty}{1}}$

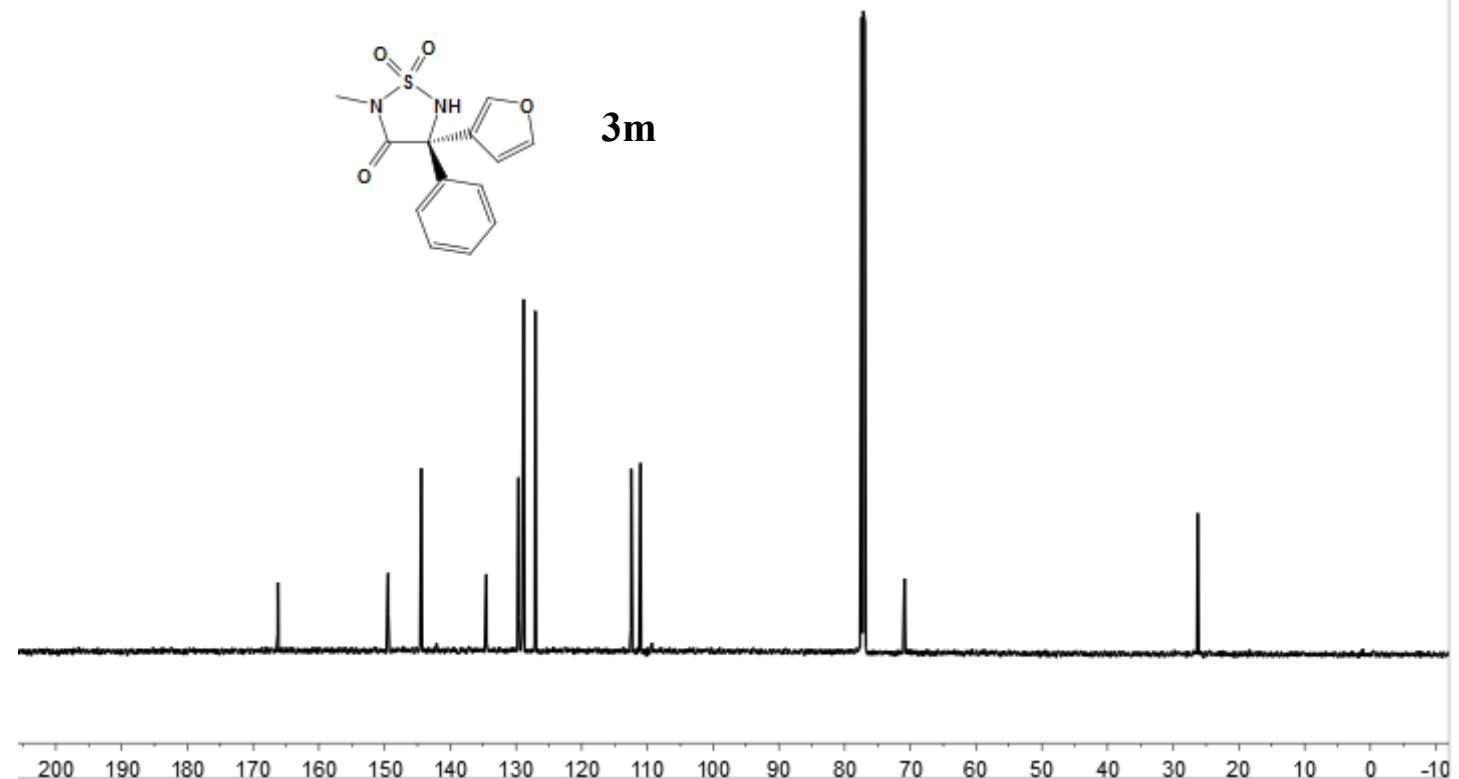



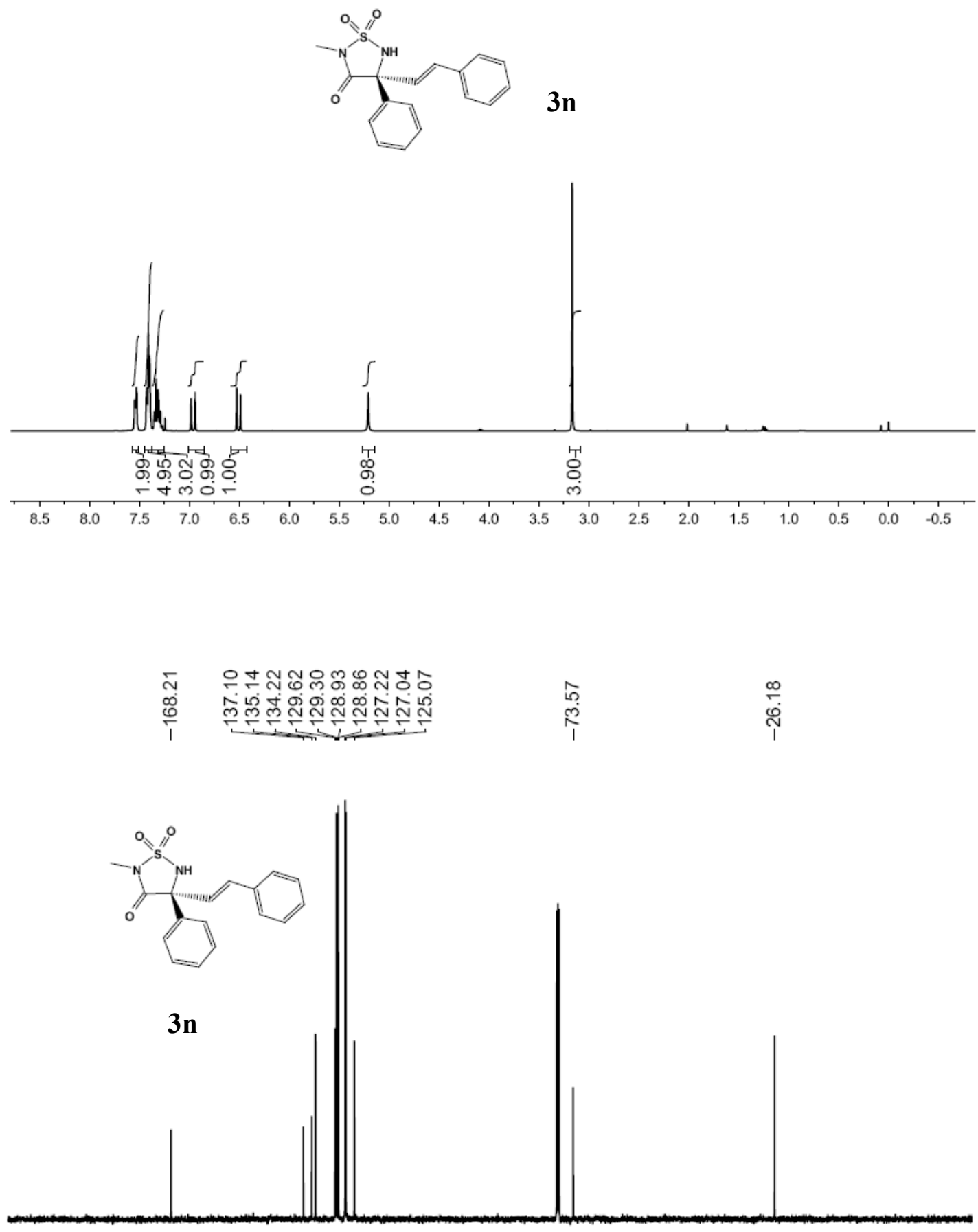

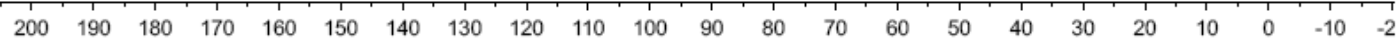




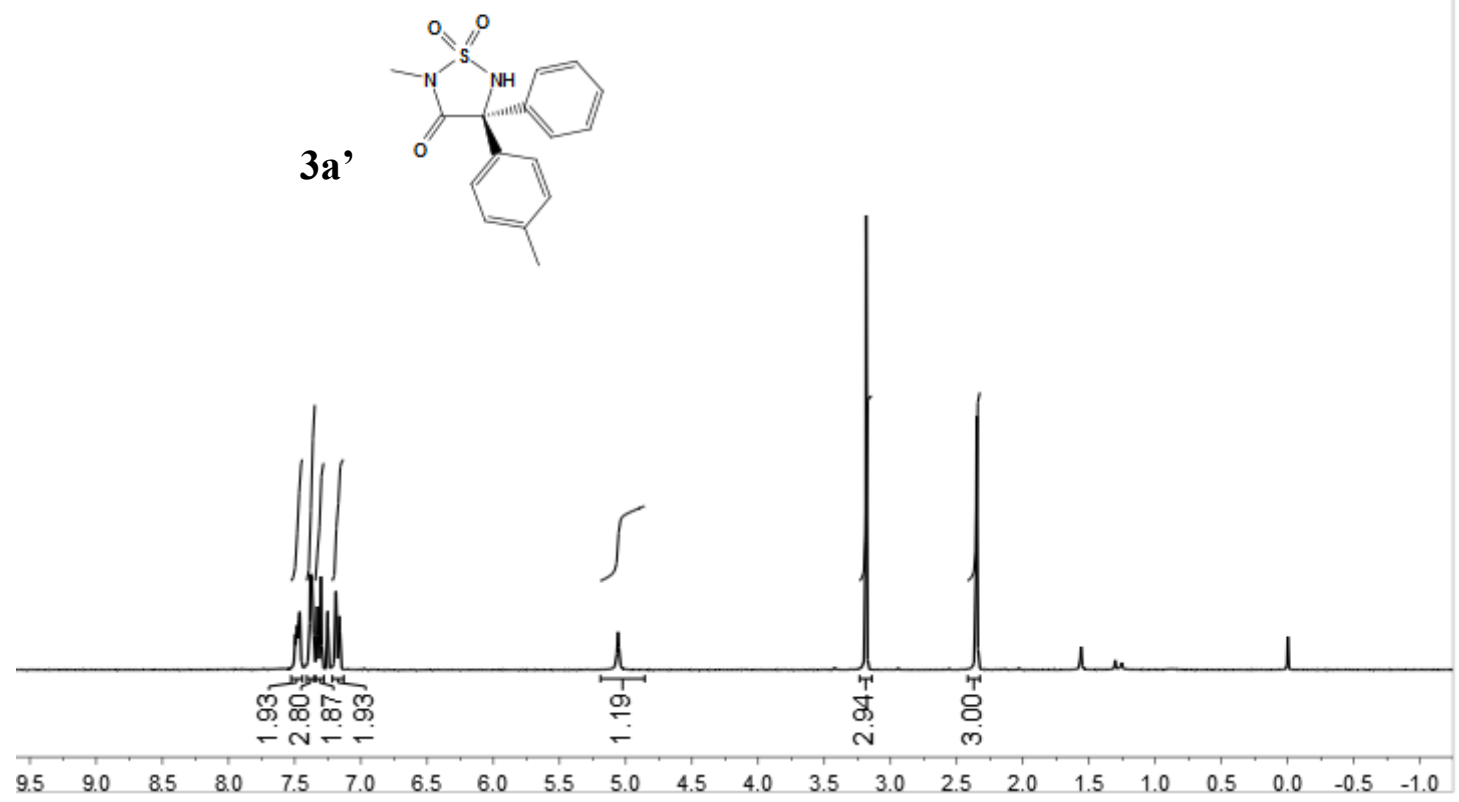

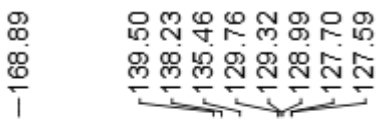

m

$\stackrel{i}{\sim}$

$3 \mathbf{a}^{\prime}$<smiles>Cc1ccc([C@]2(c3ccccc3)NS(=O)(=O)N(C)C2=O)cc1</smiles>

$\begin{array}{lllllllllllllllllllllllllll}200 & 190 & 180 & 170 & 160 & 150 & 140 & 130 & 120 & 110 & 100 & 90 & 80 & 70 & 60 & 50 & 40 & 30 & 20 & 10 & 0\end{array}$ 


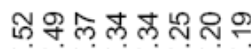

NiniNiñ

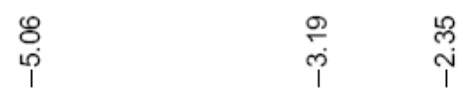
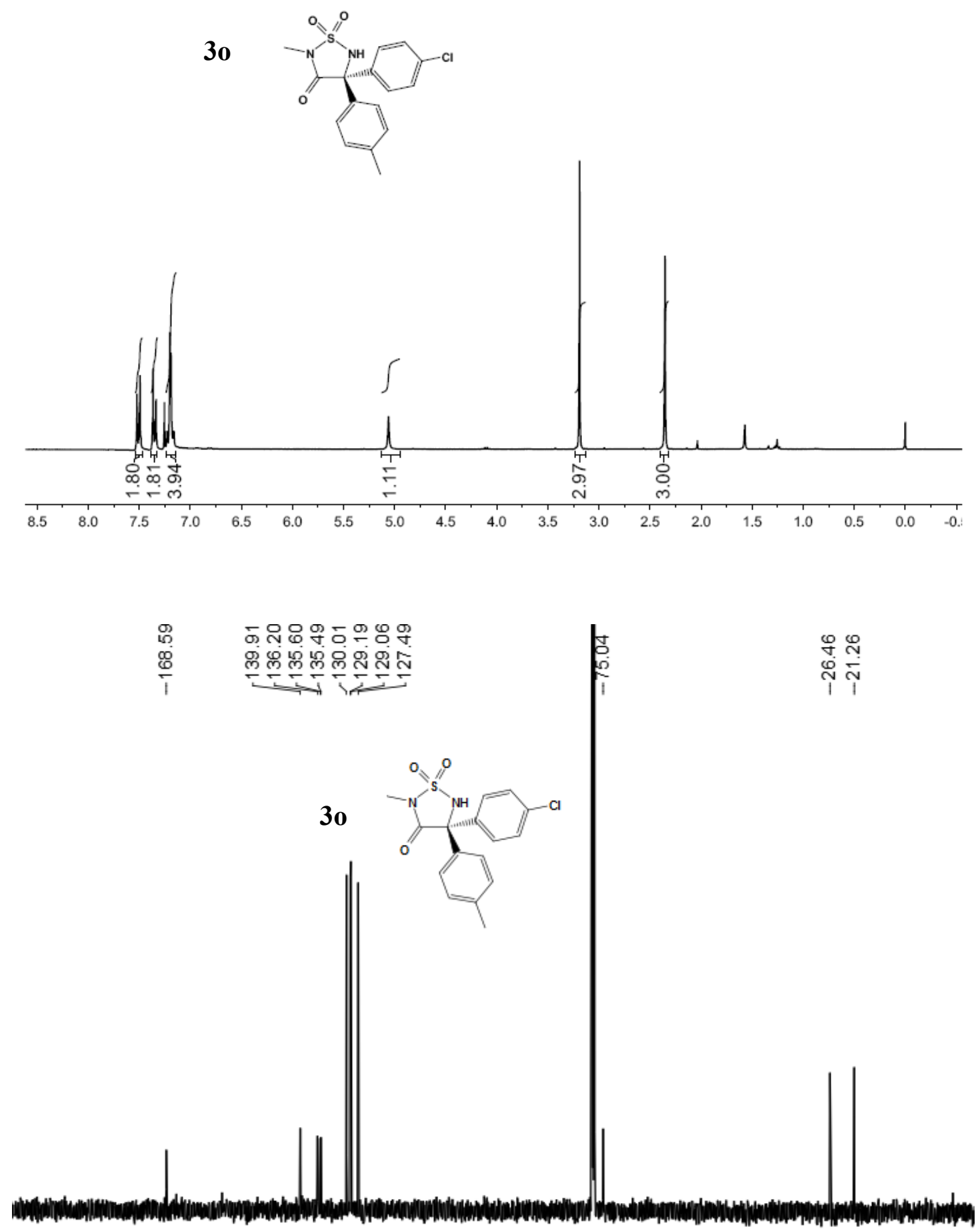

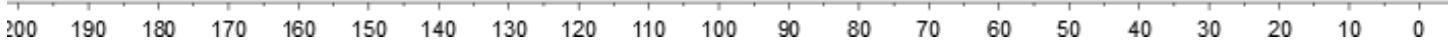




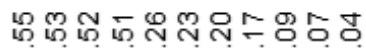

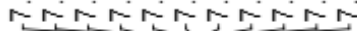

$\stackrel{\circ}{\circ}$

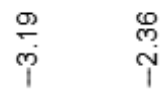
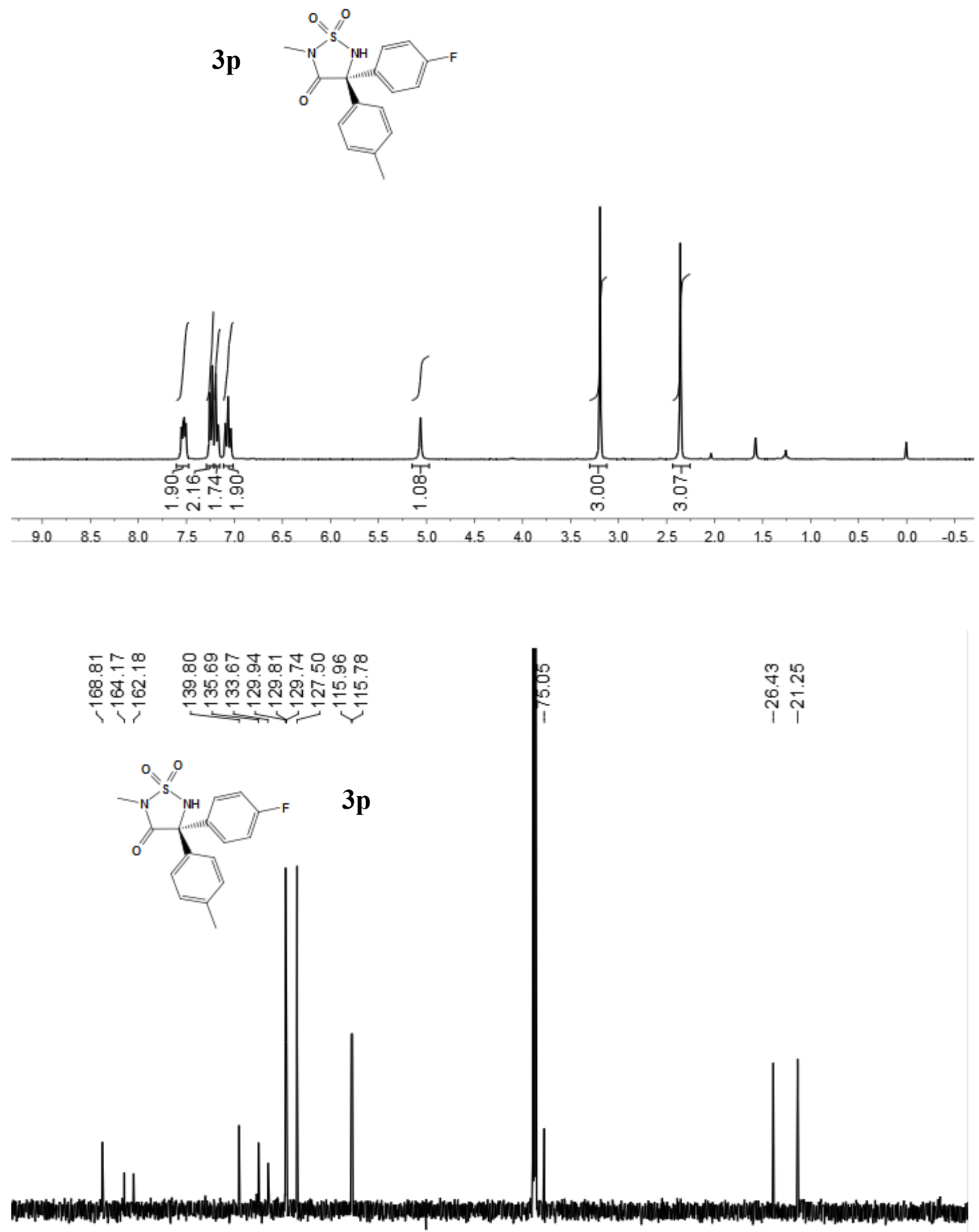

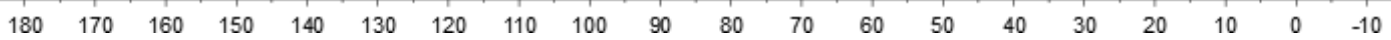




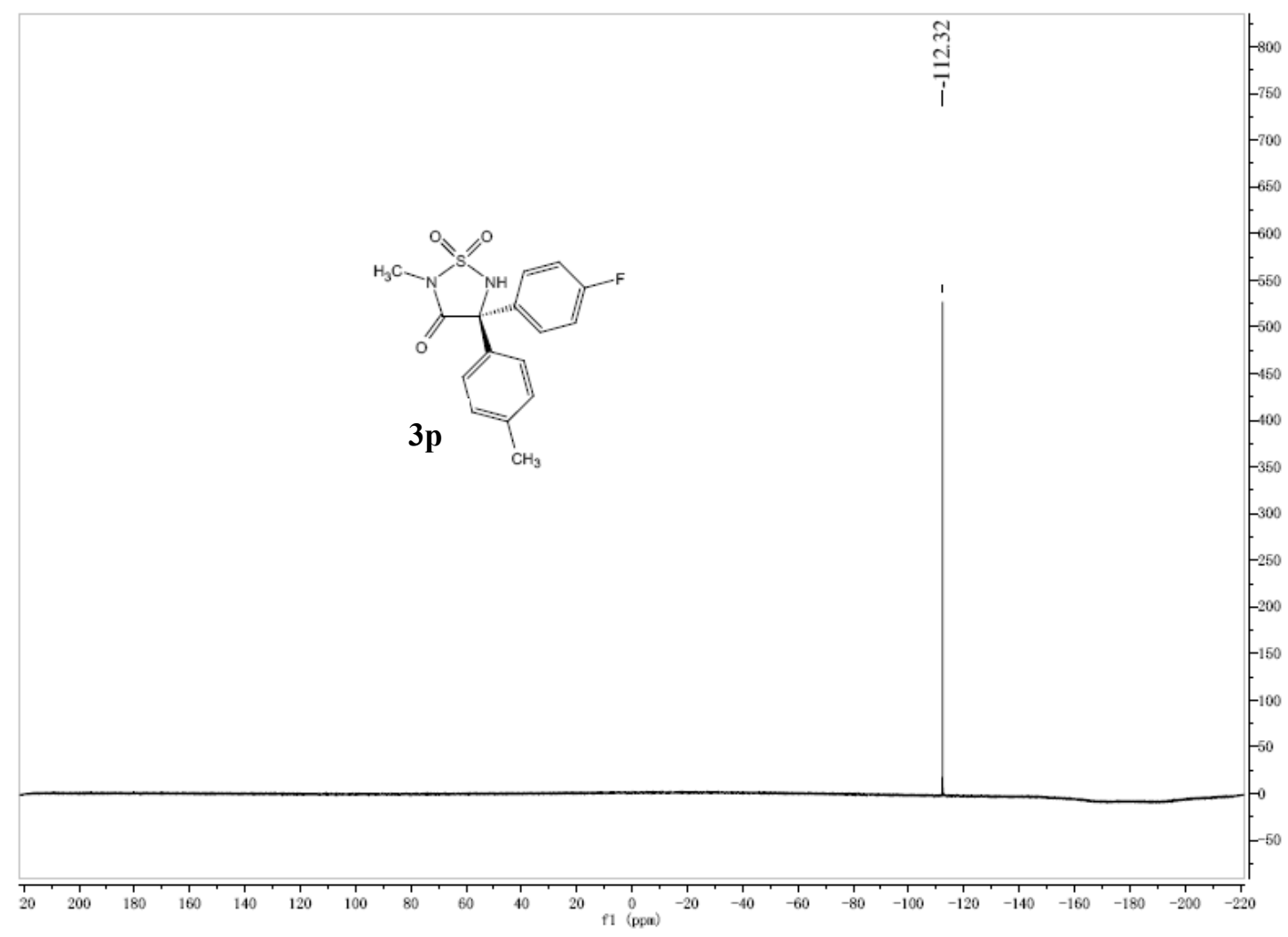

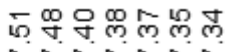

risisis
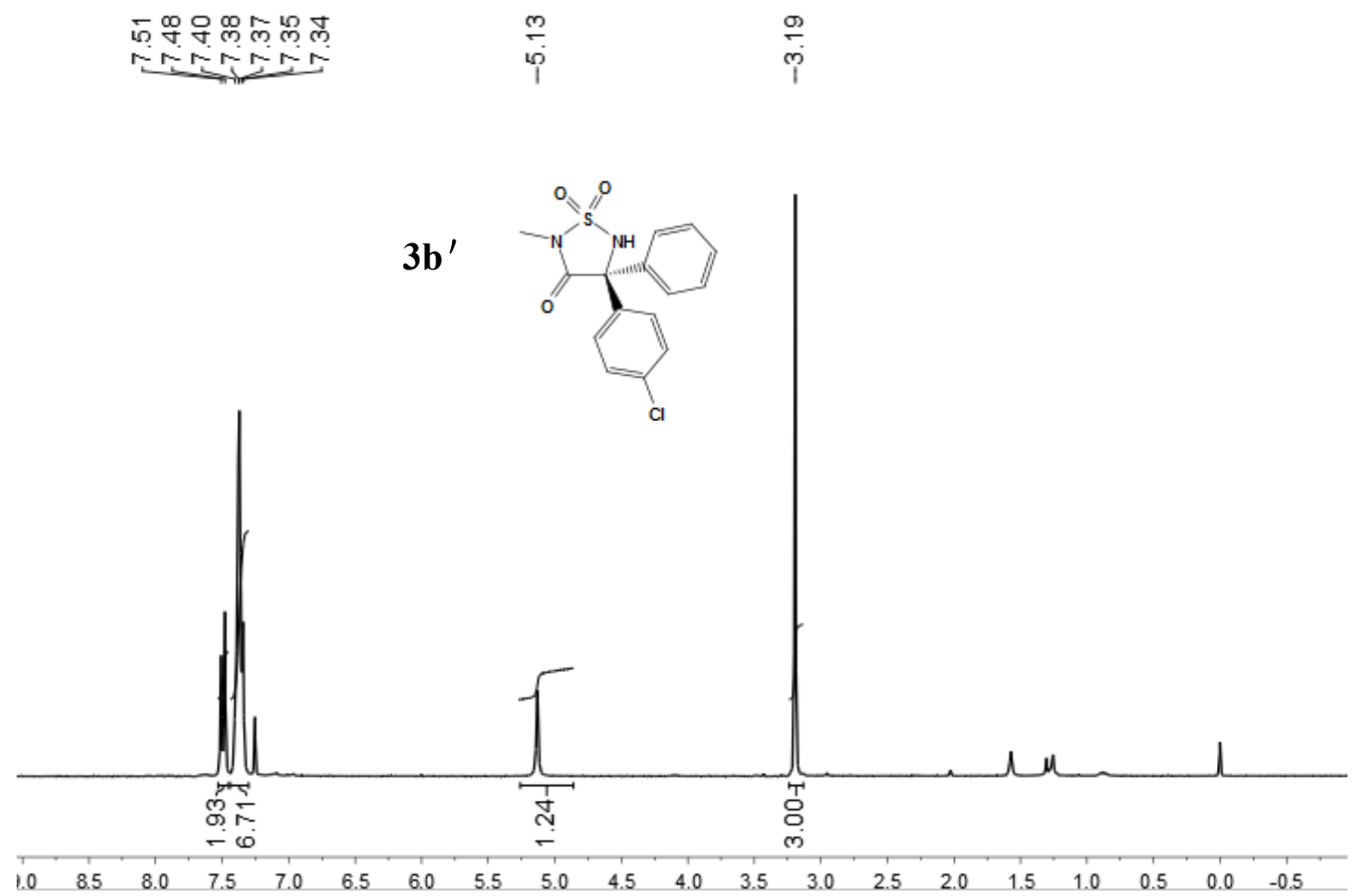

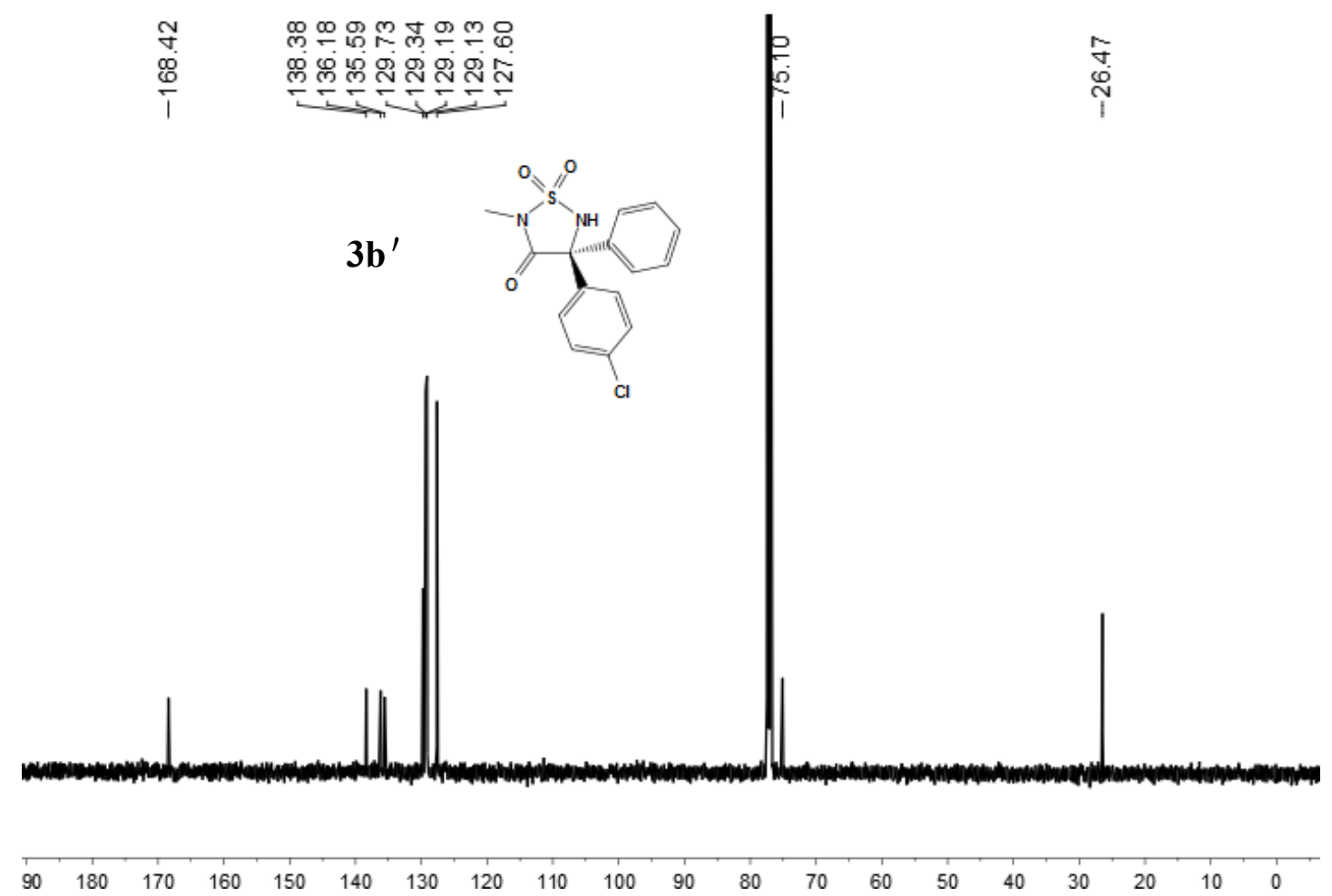

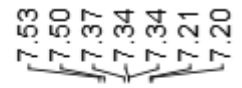
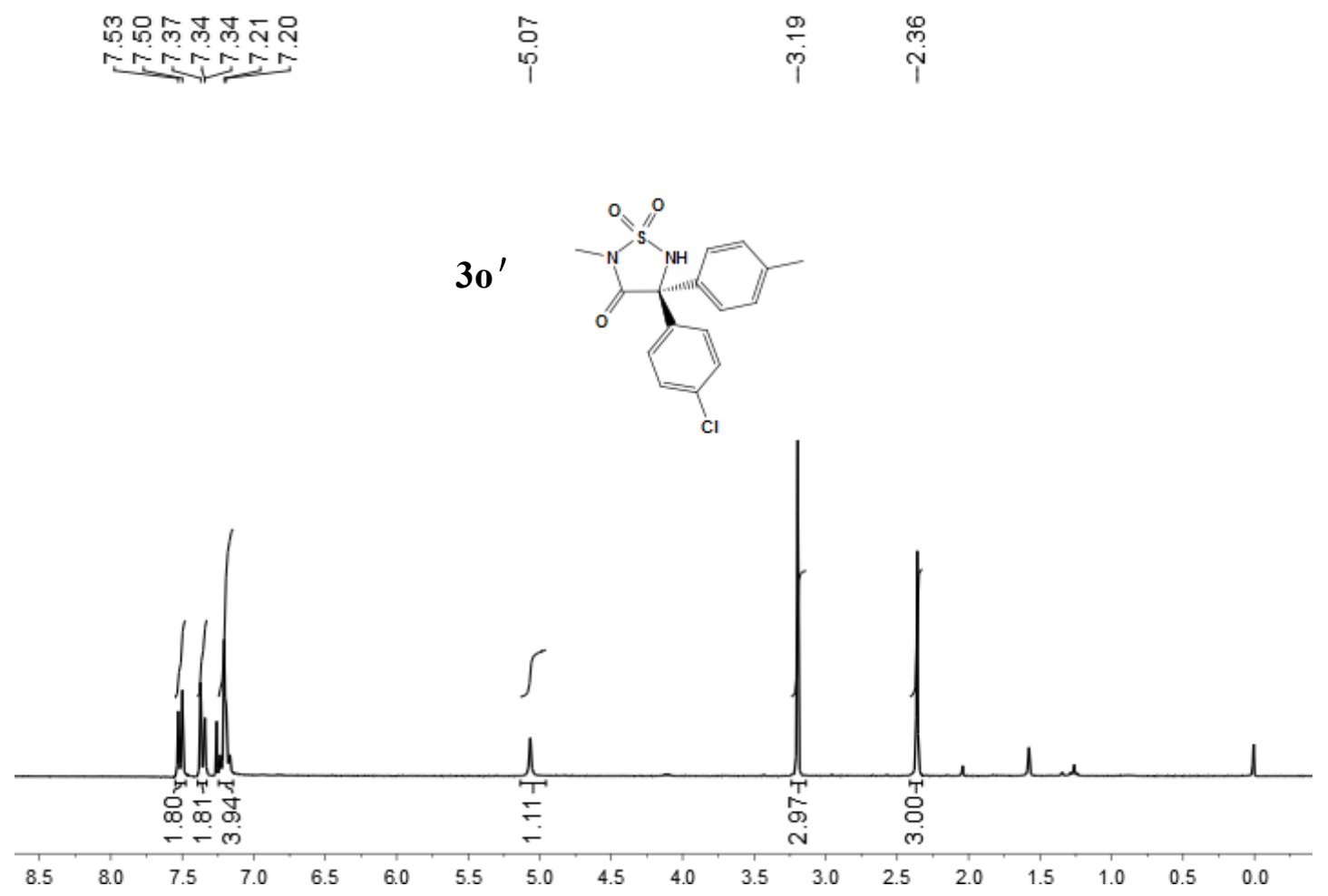


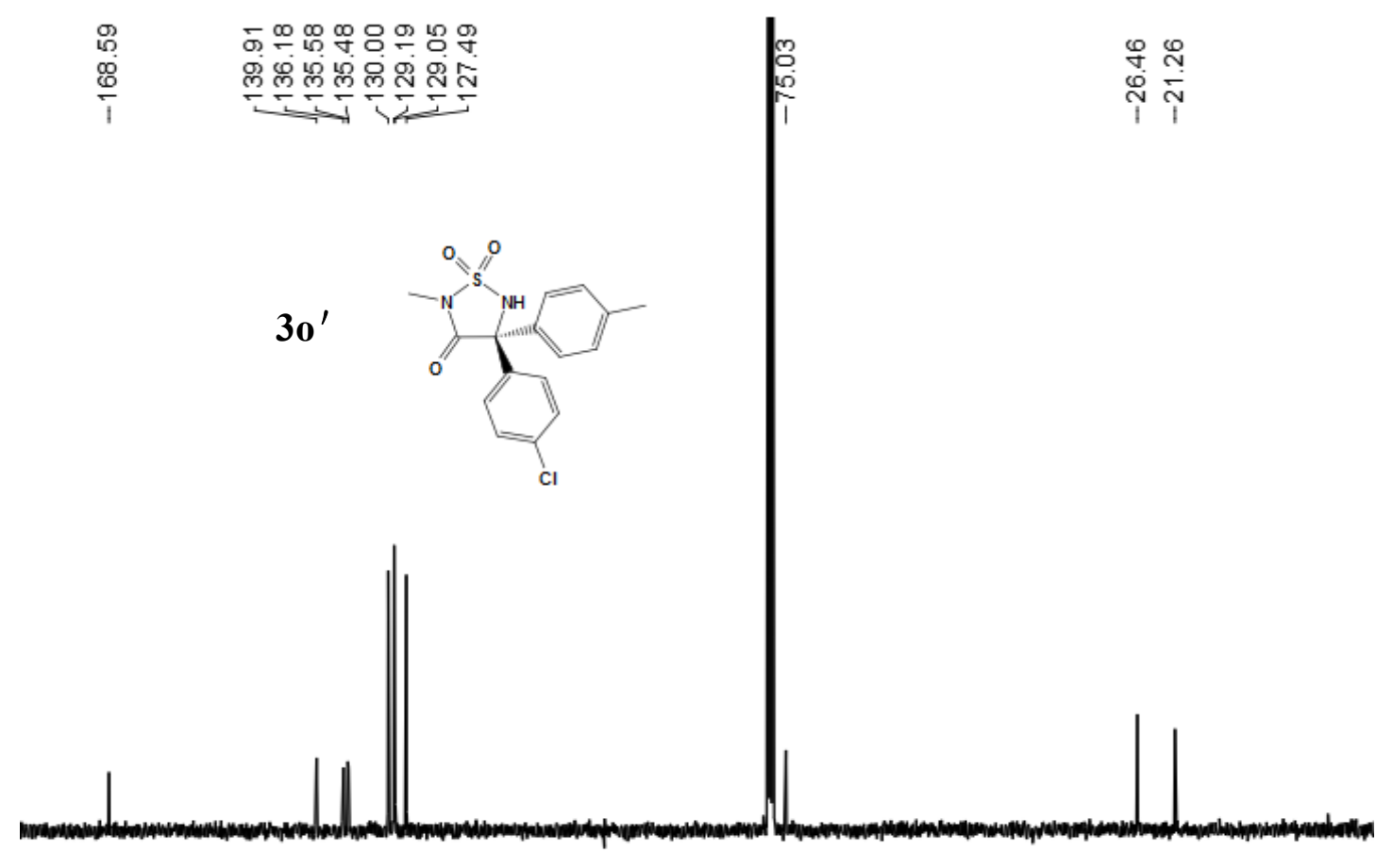

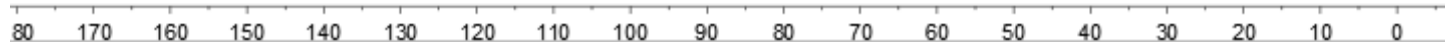

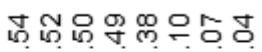

riminisin
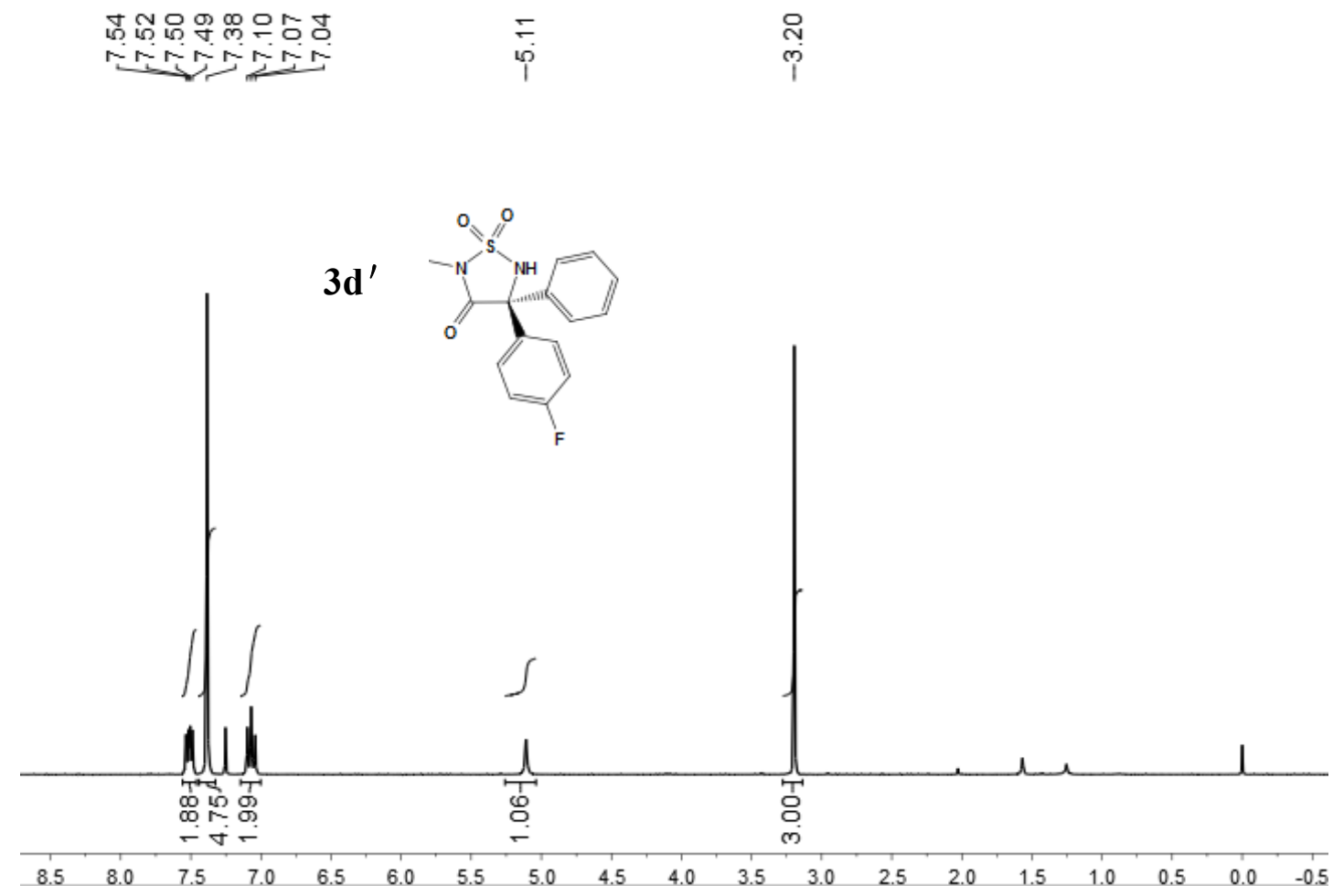


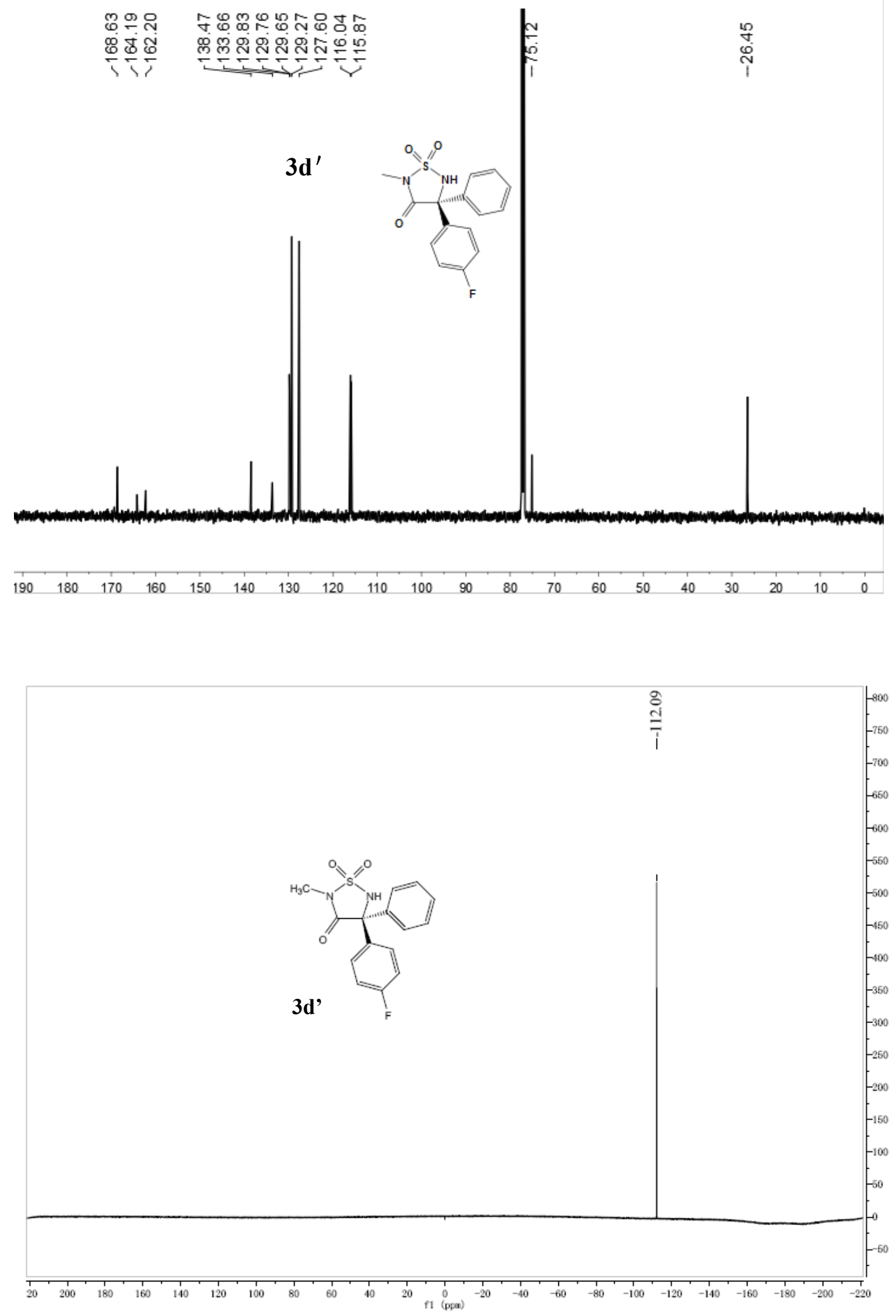



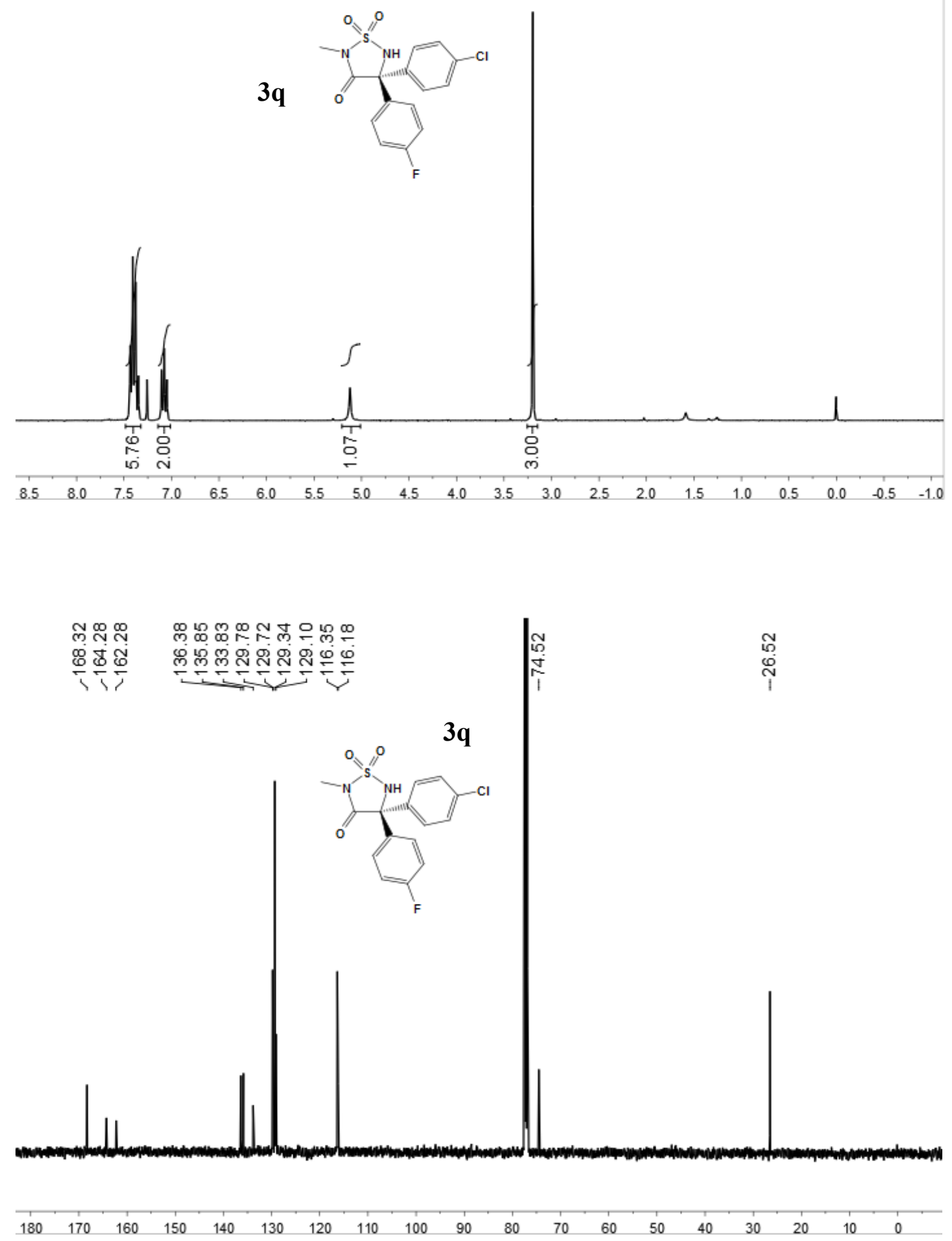


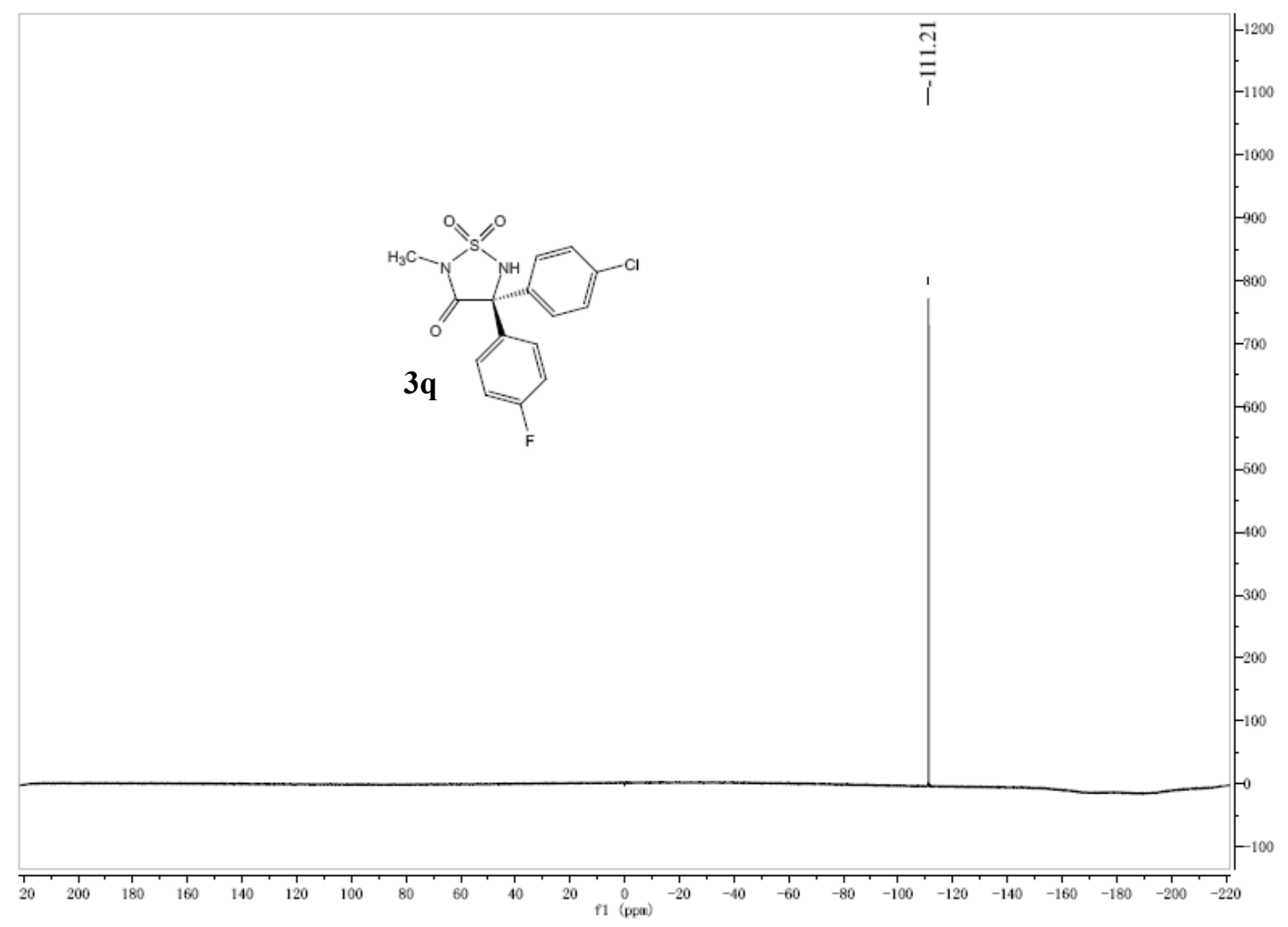

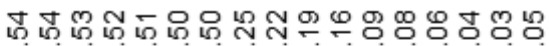

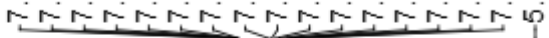

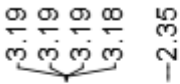

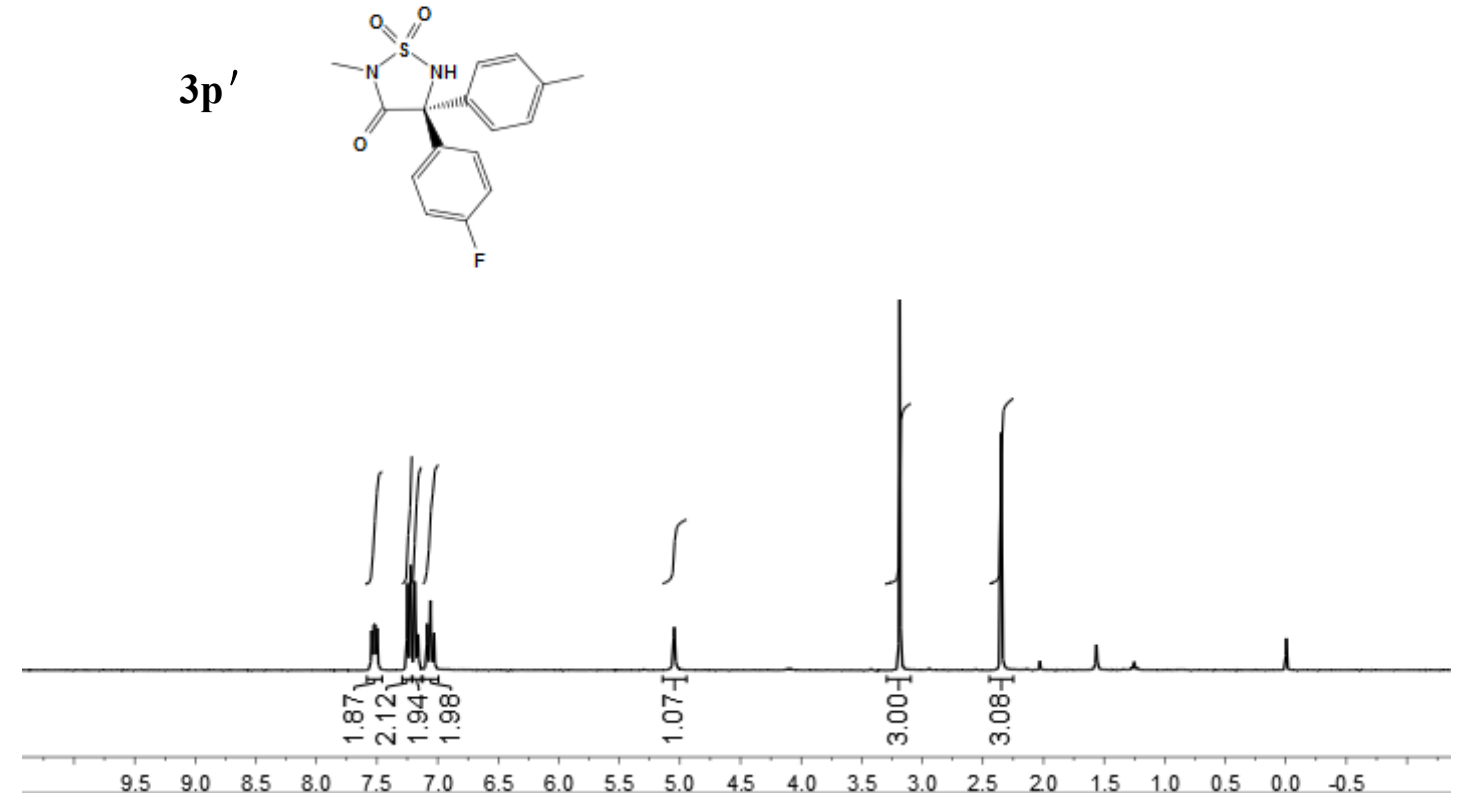



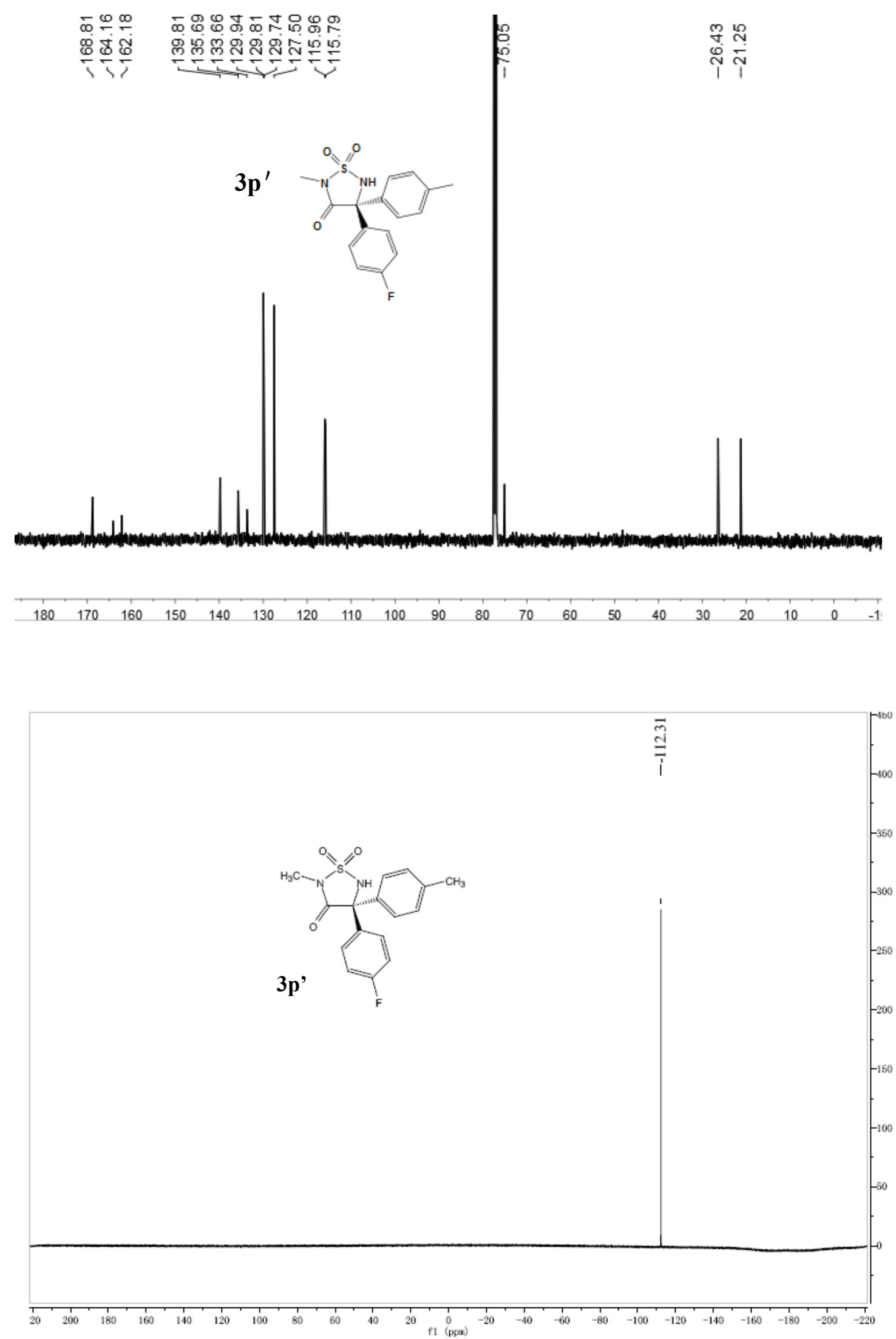

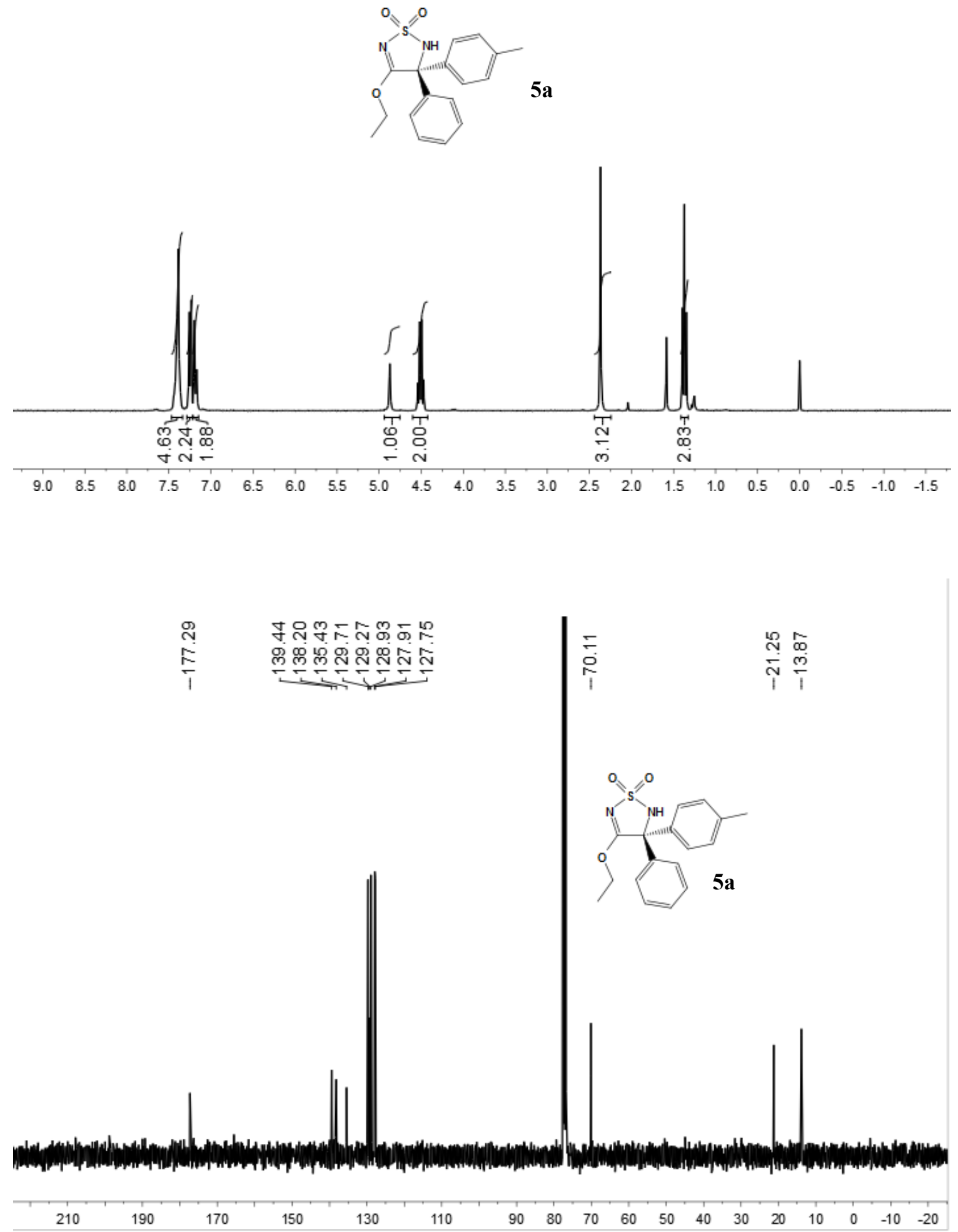

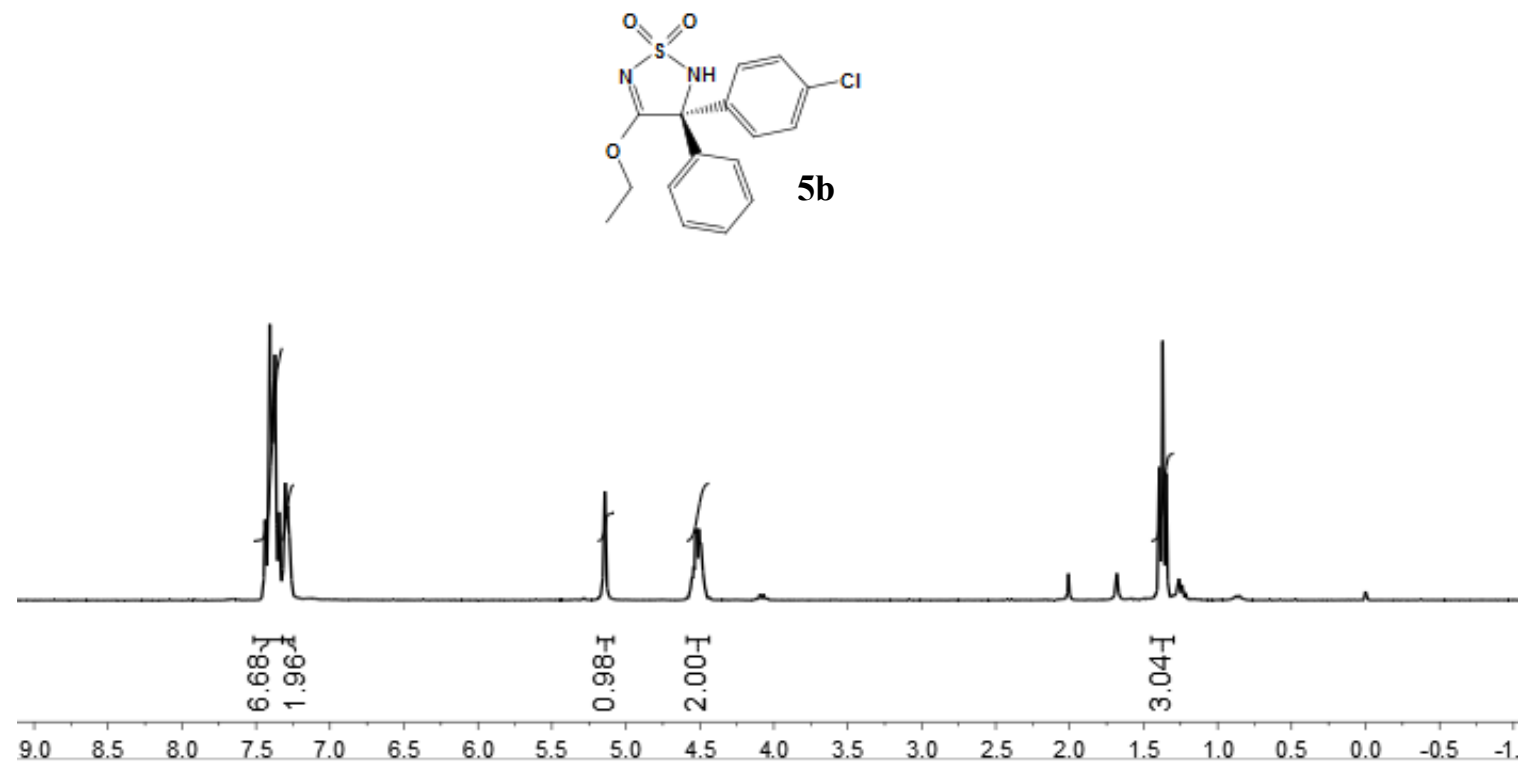

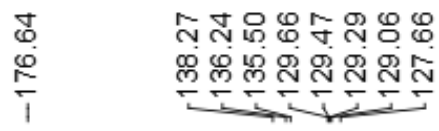

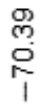

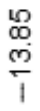
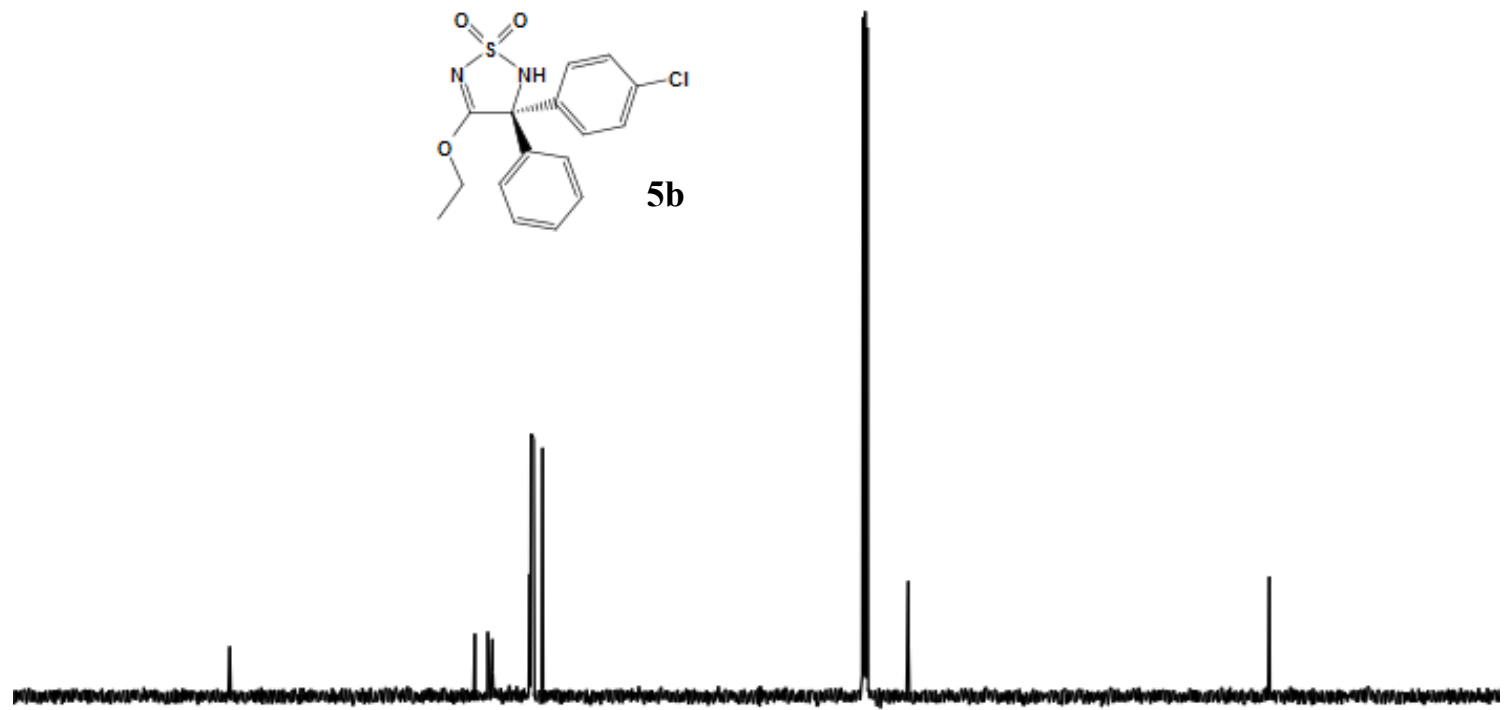

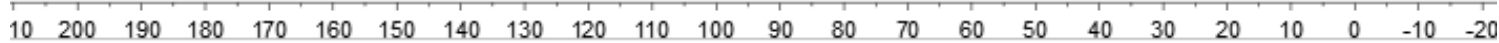



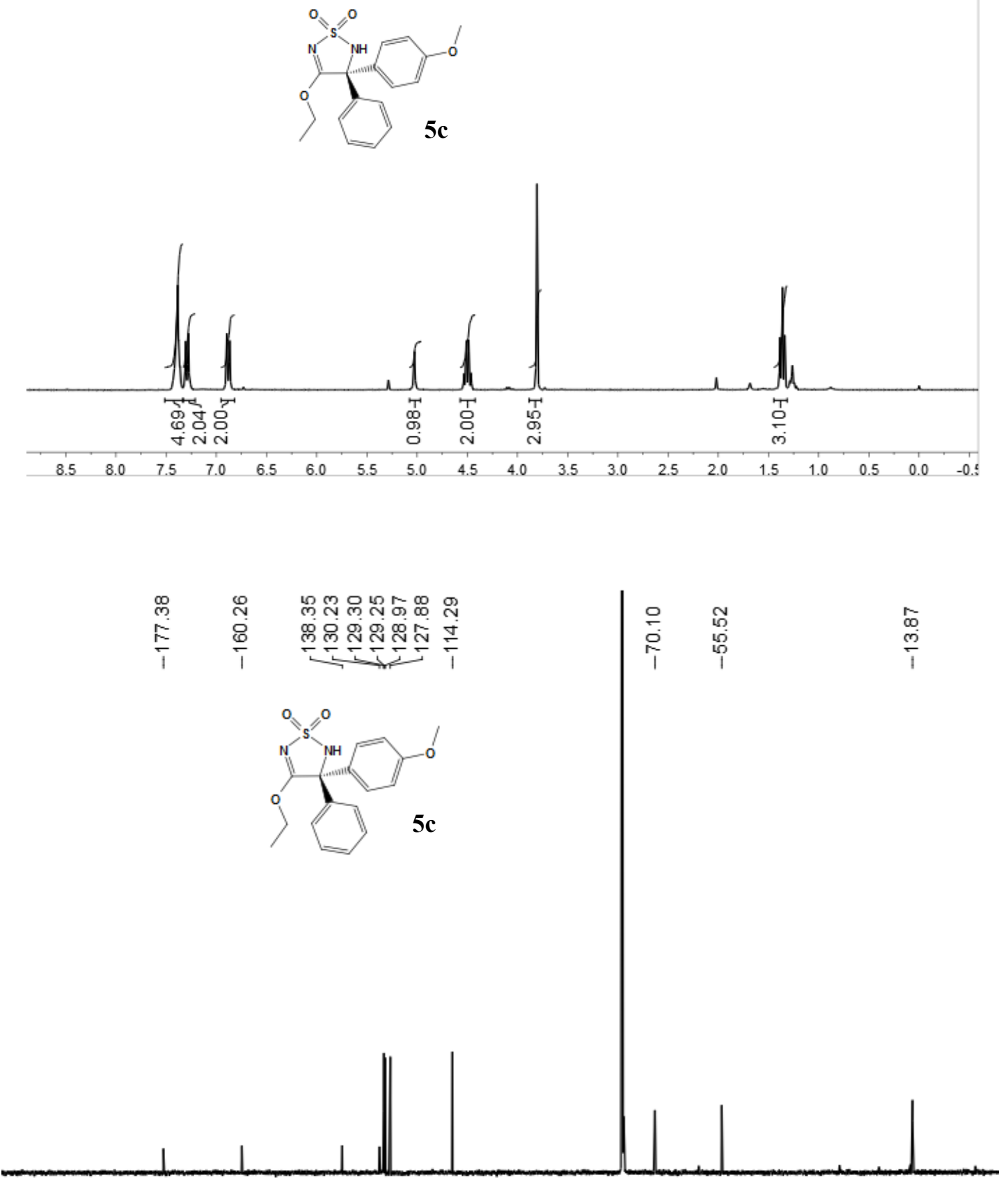

$\begin{array}{lllllllllllllllllllllllllllllll}210 & 200 & 190 & 180 & 170 & 160 & 150 & 140 & 130 & 120 & 110 & 100 & 90 & 80 & 70 & 60 & 50 & 40 & 30 & 20 & 10 & 0\end{array}$ 


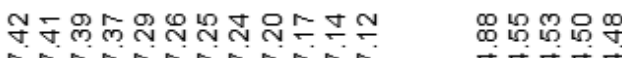

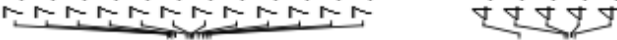

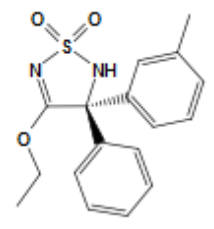

5d

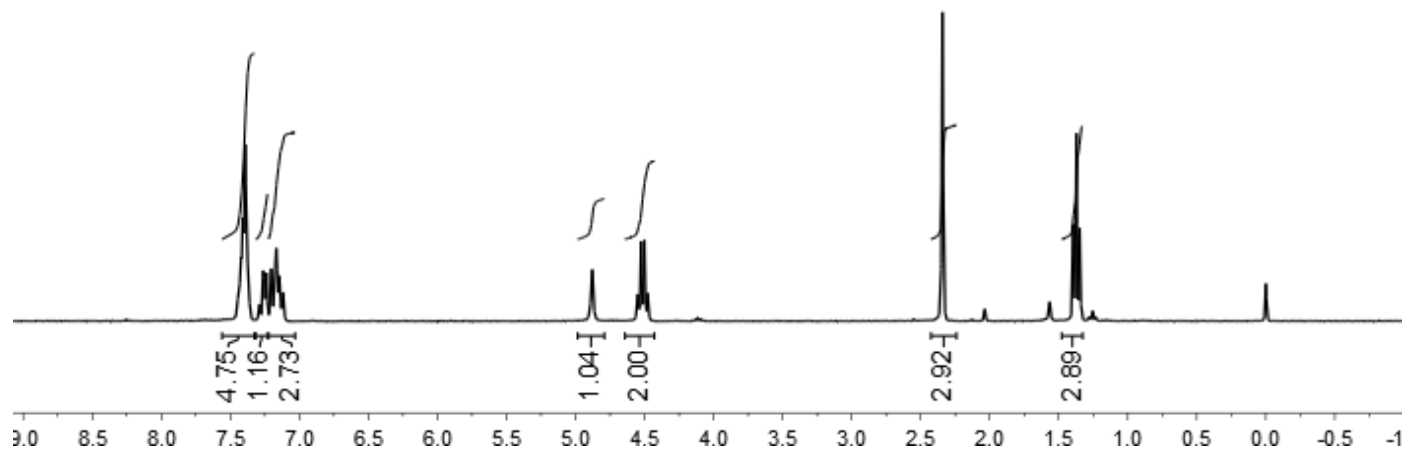

$\frac{\infty}{5}$

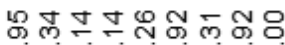

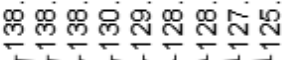

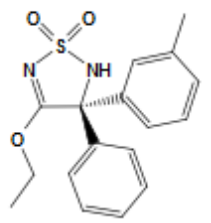

5d

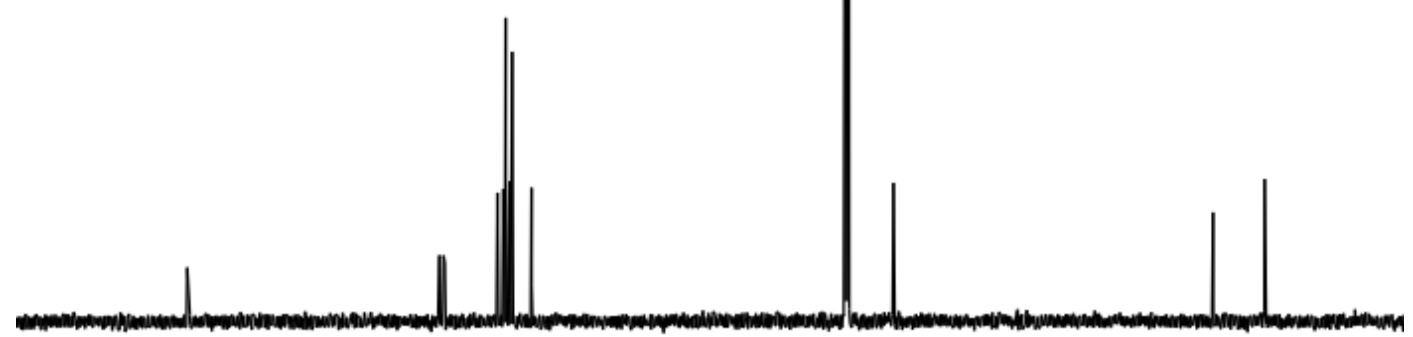

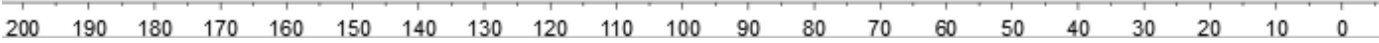




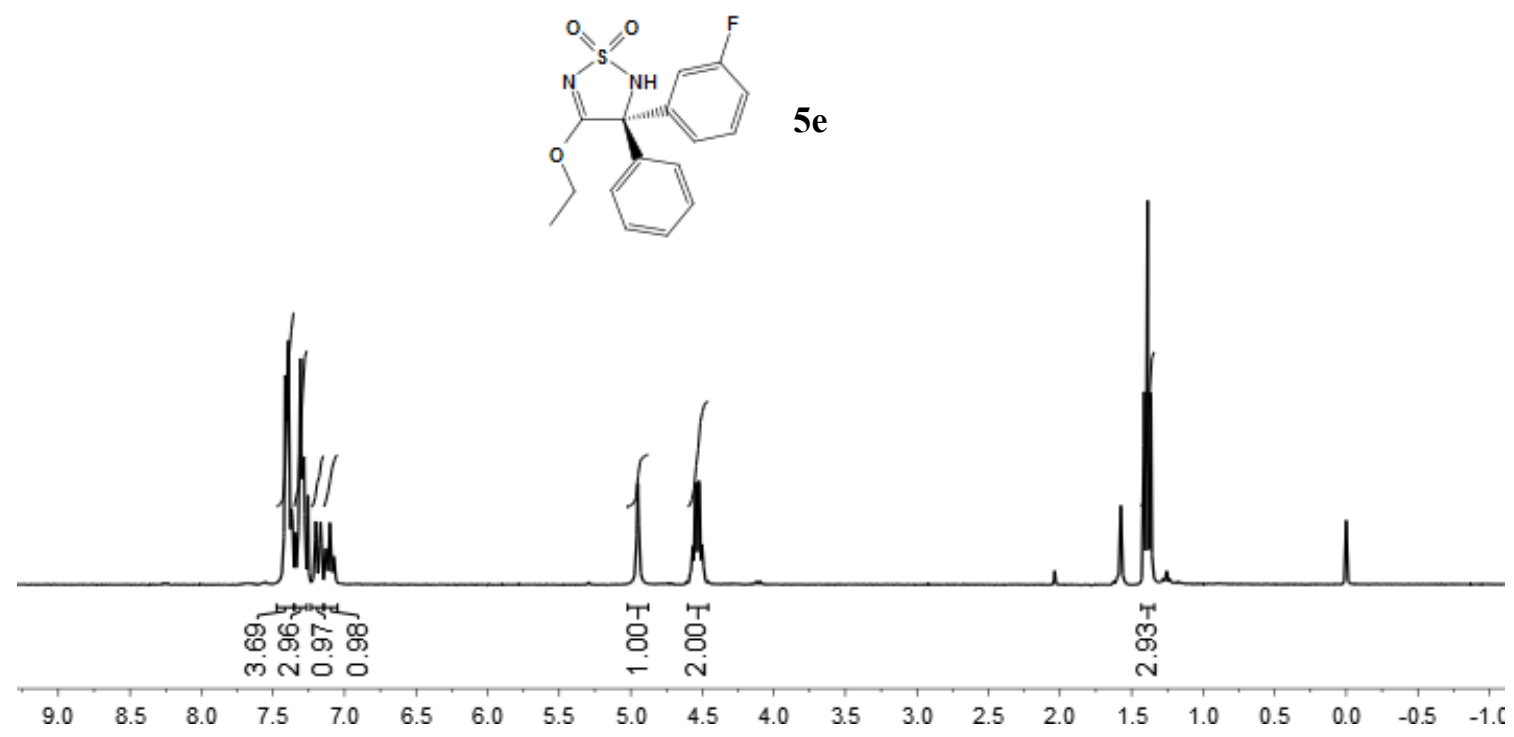

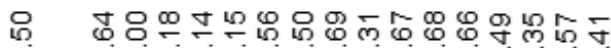

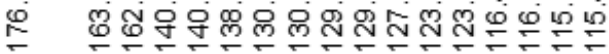

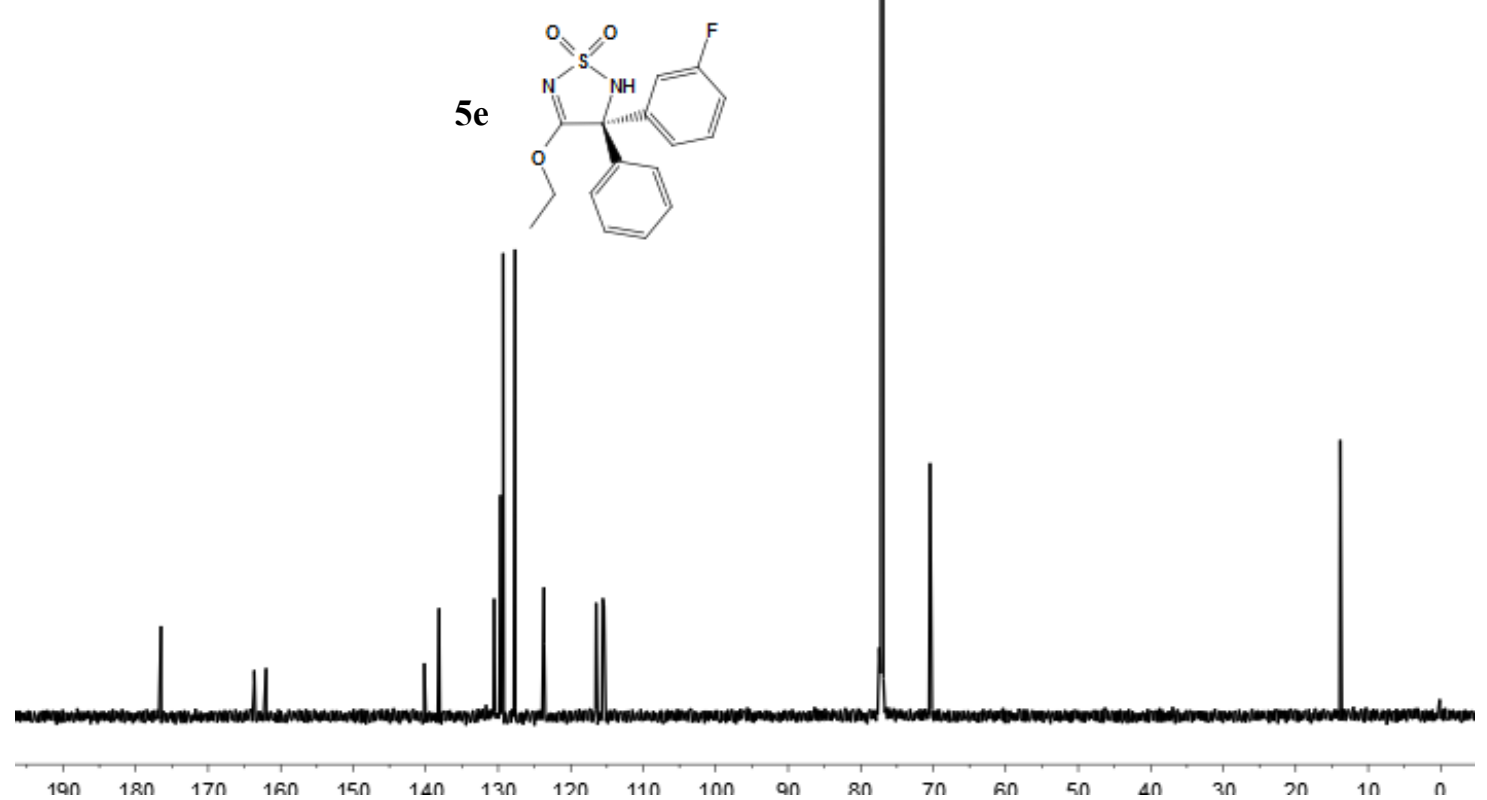




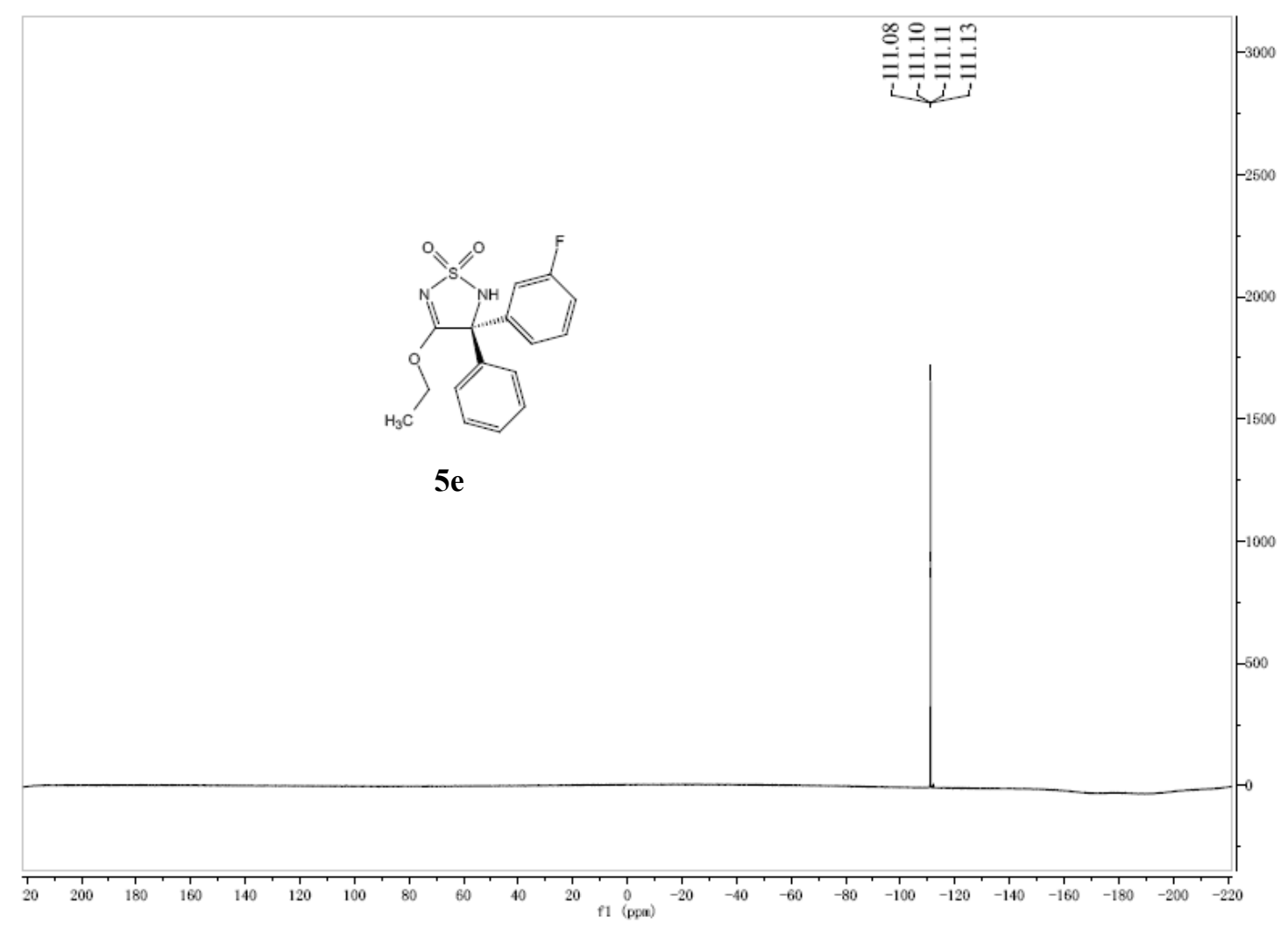

రำ

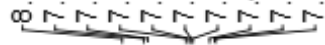

ธ్ర

化过

分
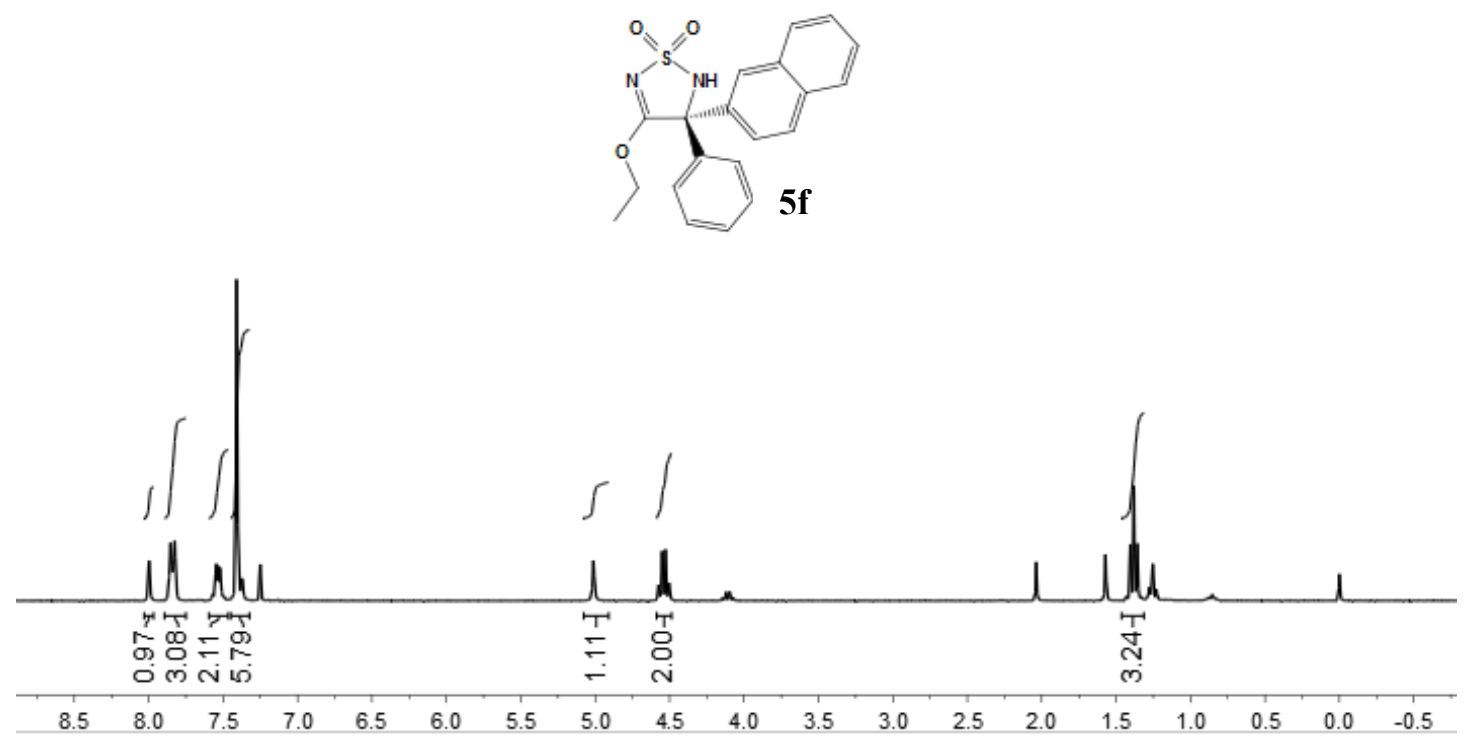


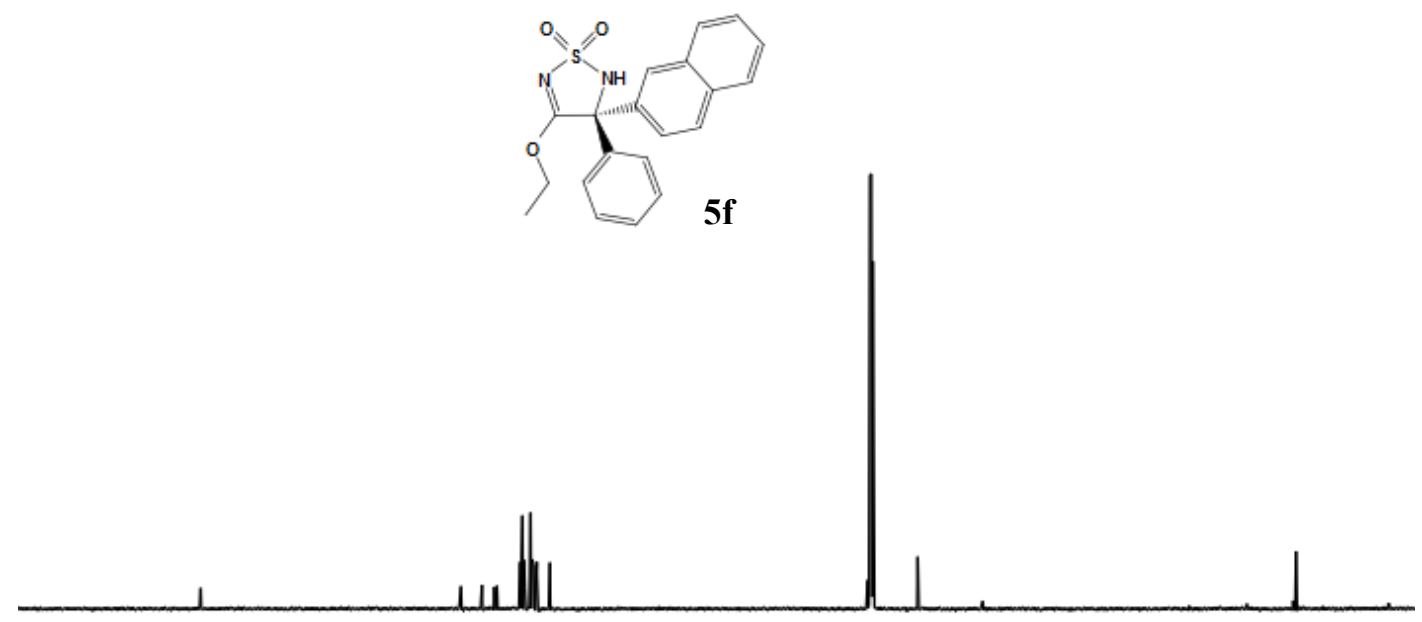

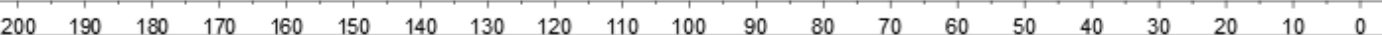

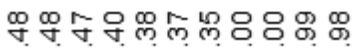

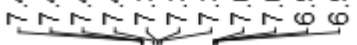

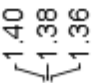
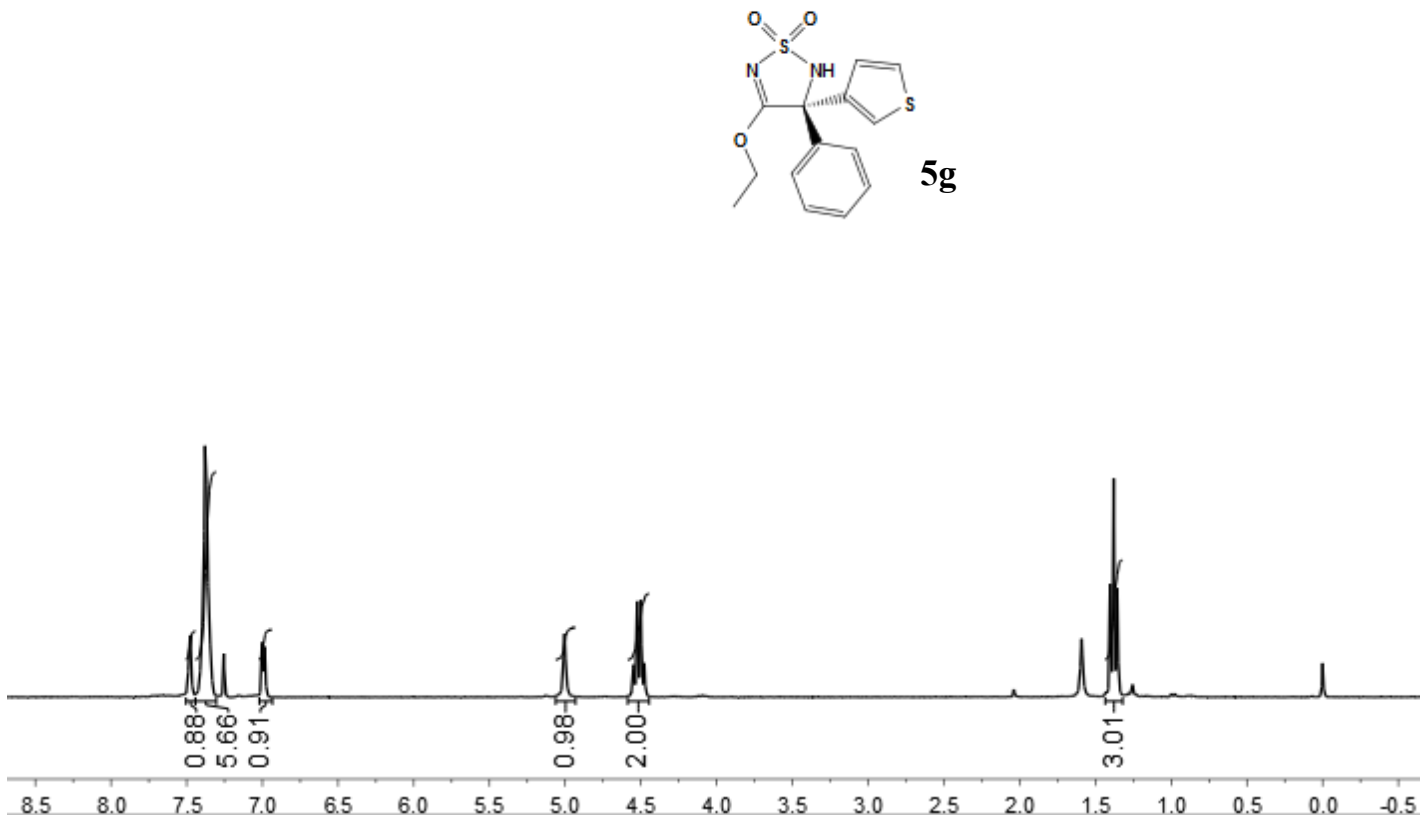


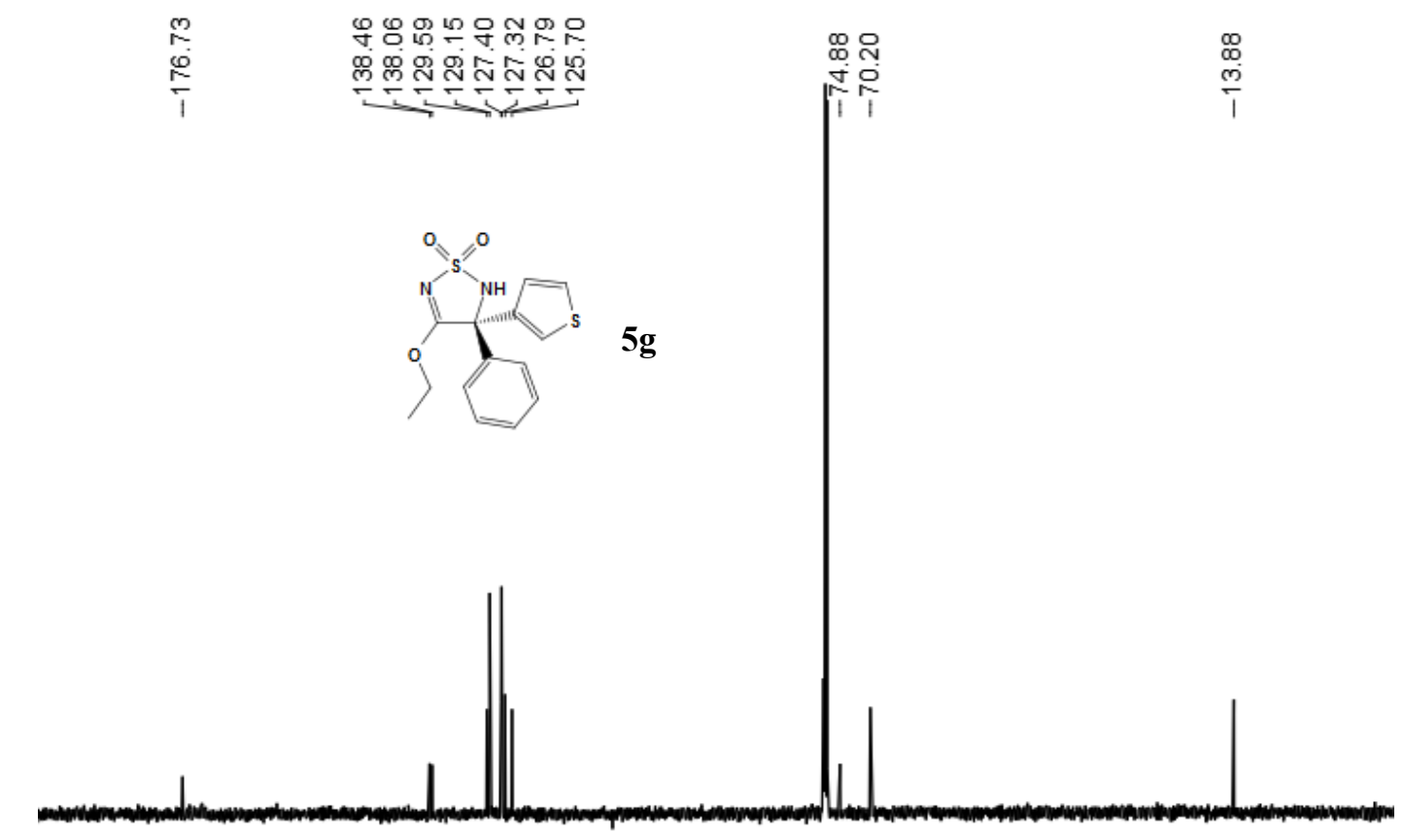

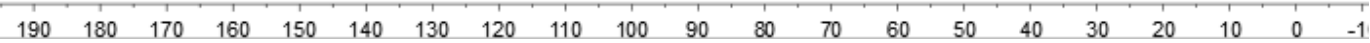

mై

$\sim m m i n$

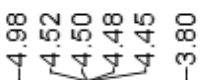

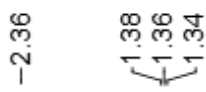
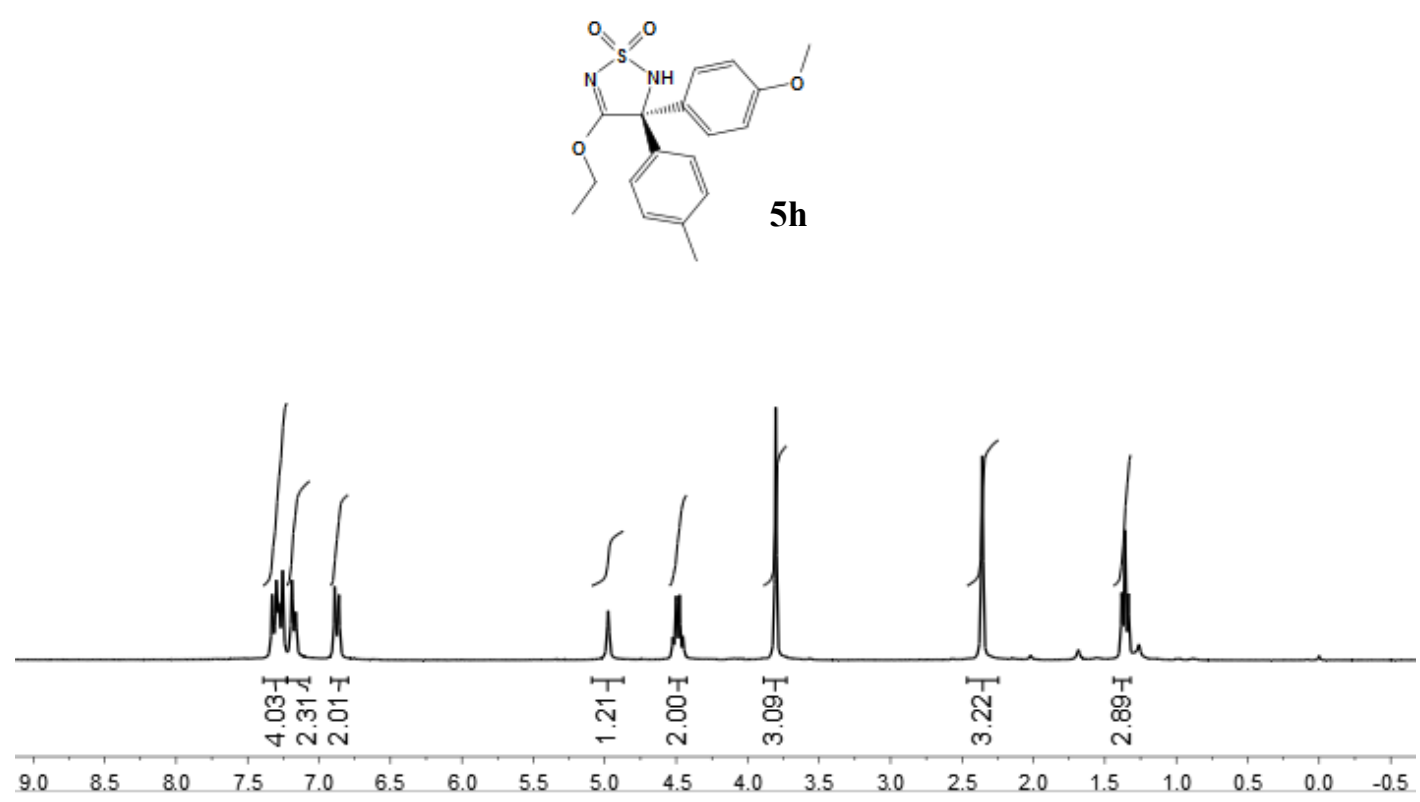

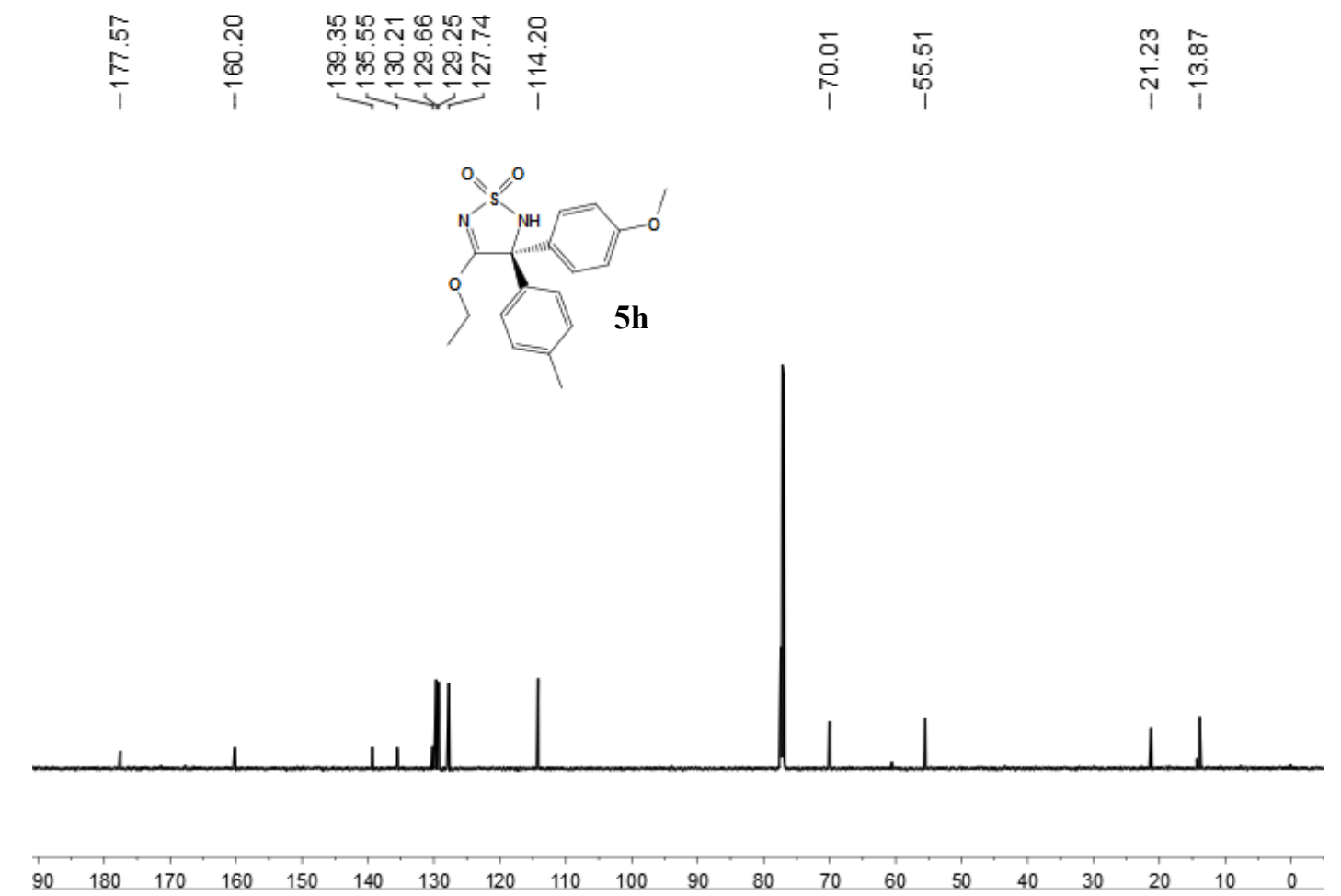

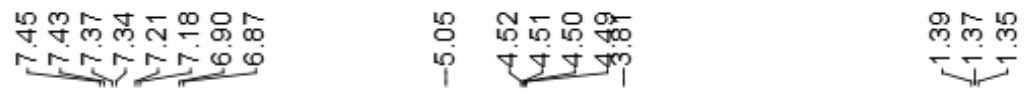
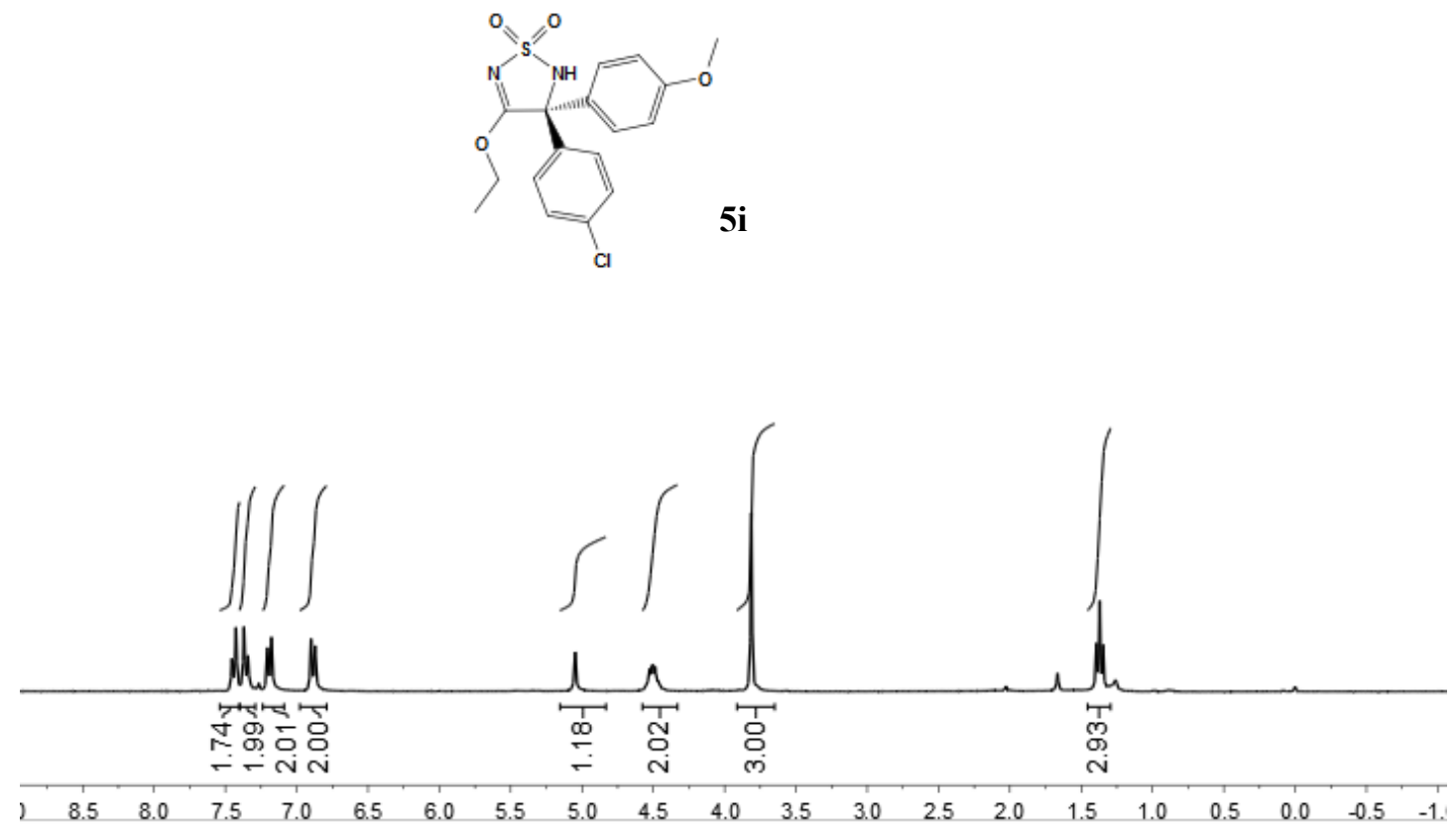


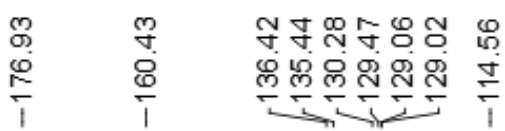
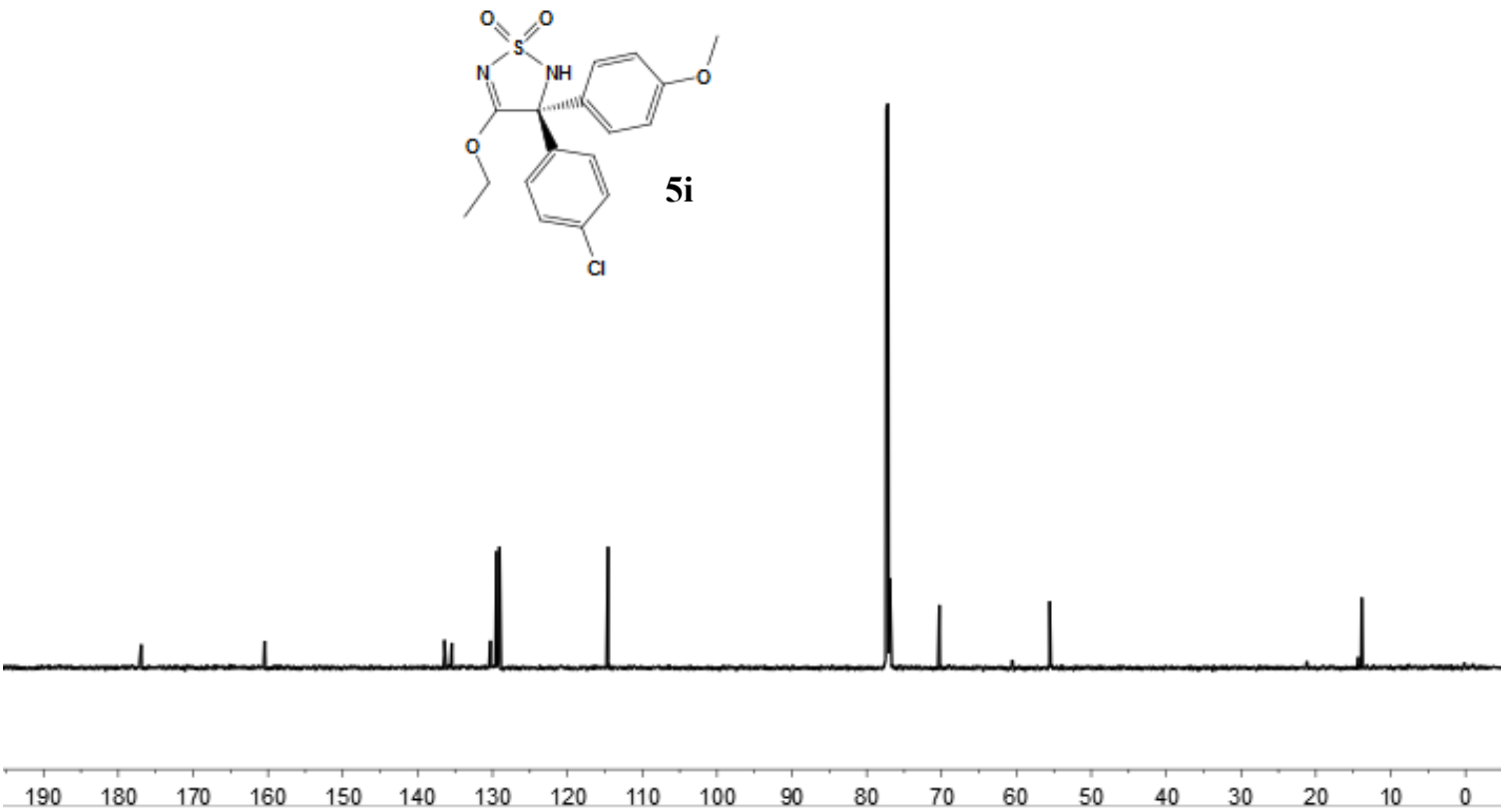

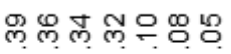

Mrmm

梁

守
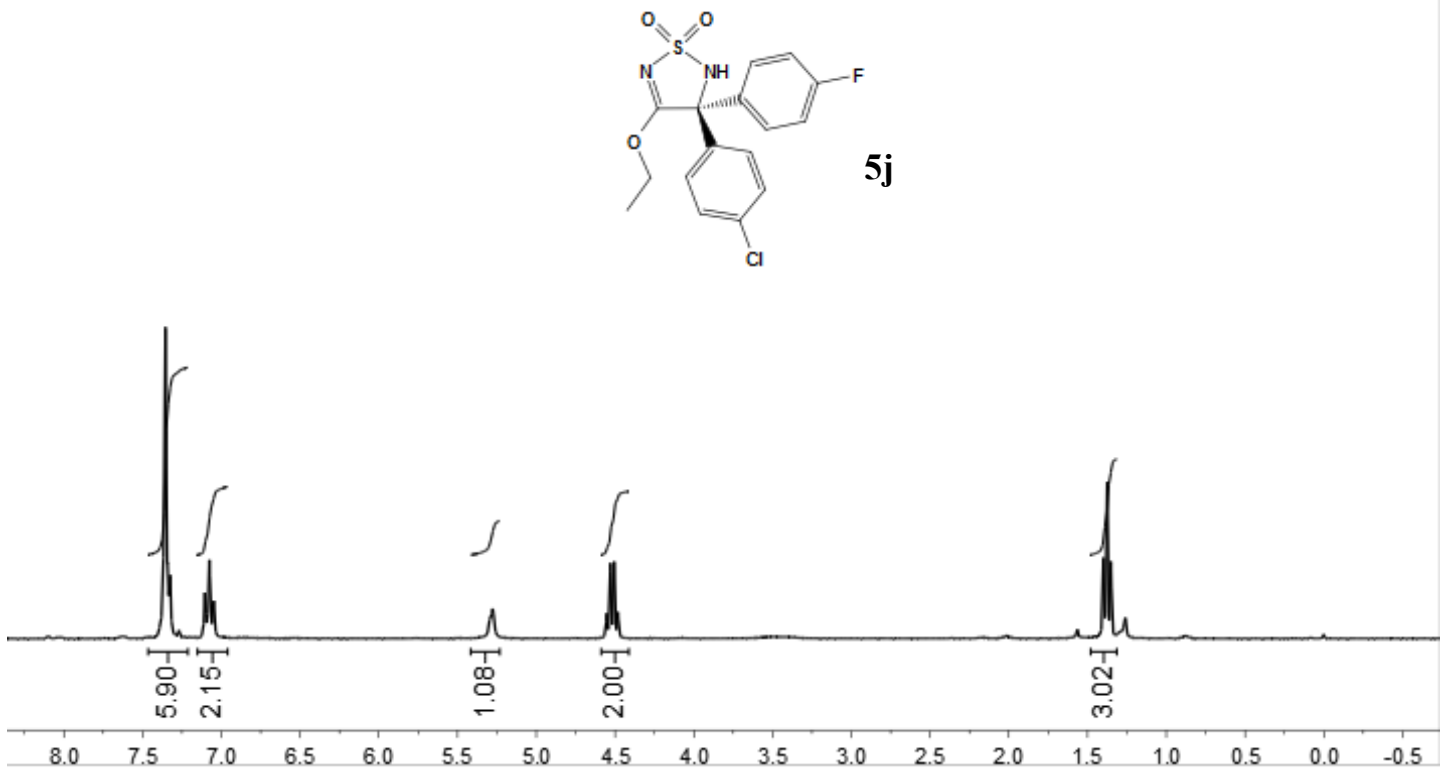


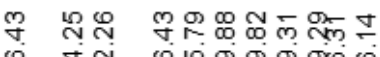

웅

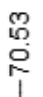

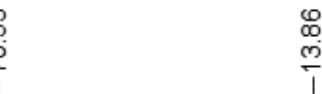
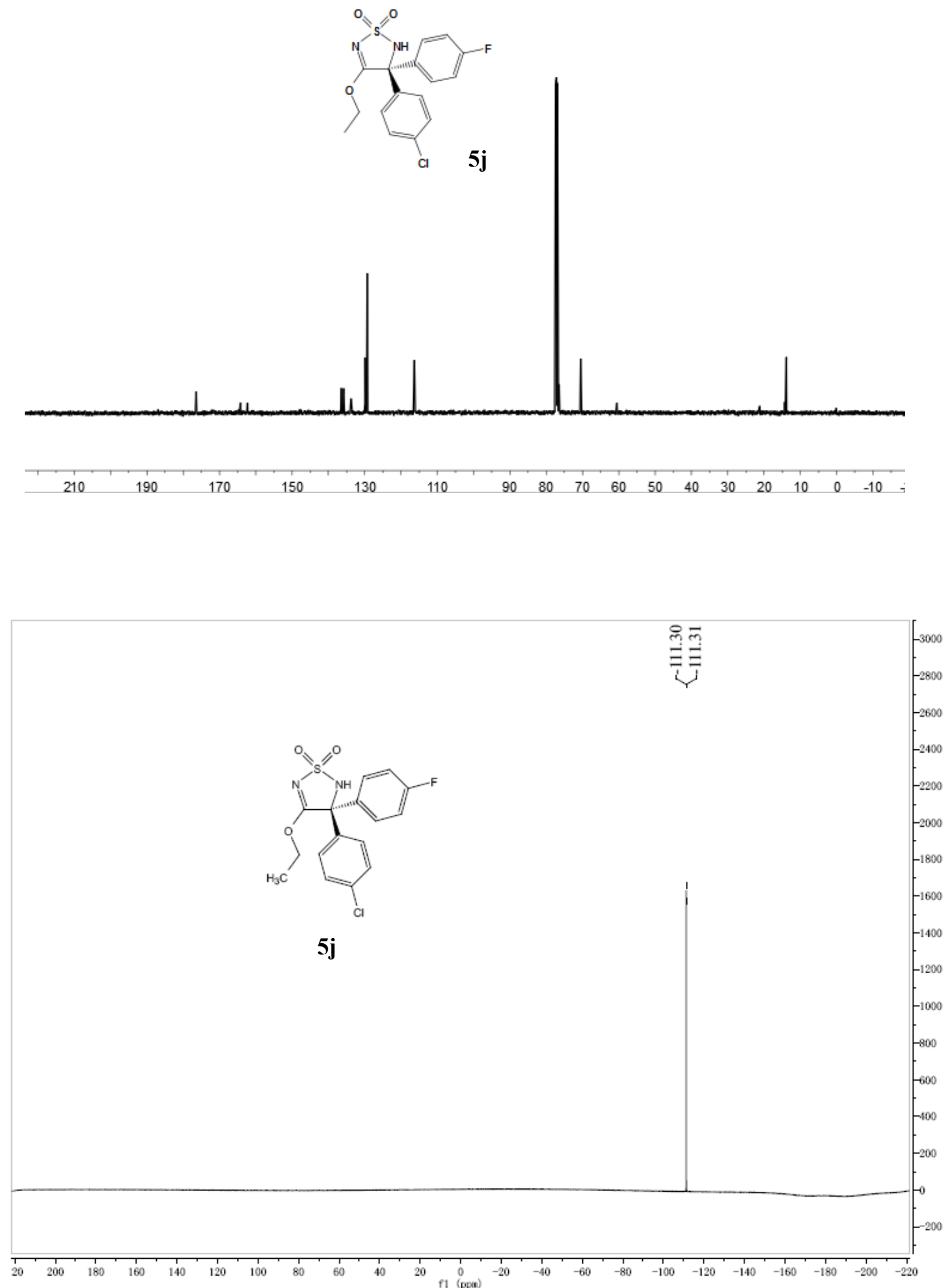


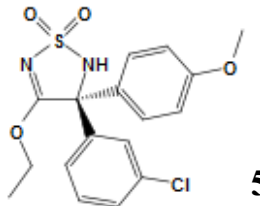

$5 \mathbf{k}$

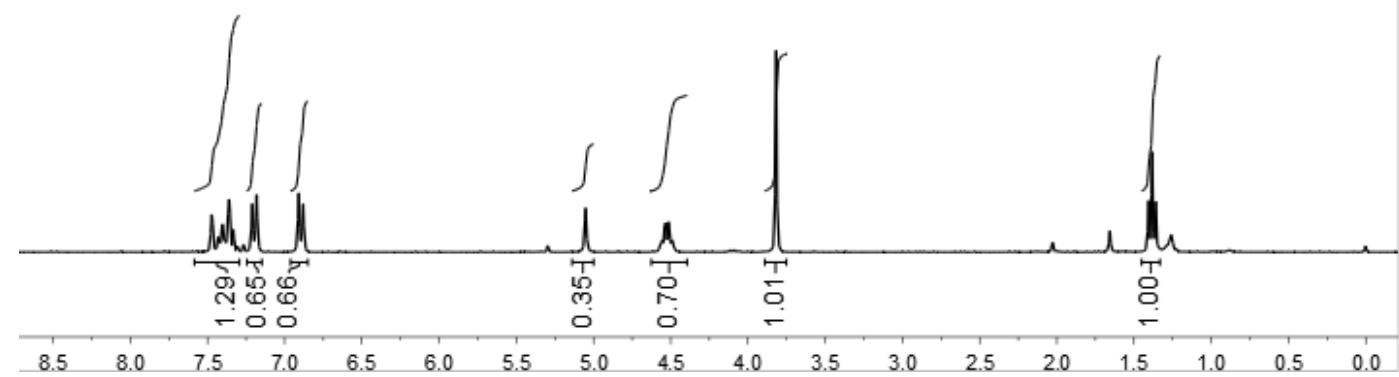

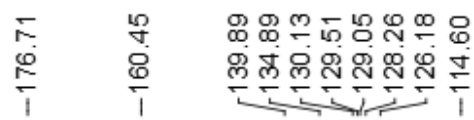

$\begin{array}{ll}m & 0 \\ 0 & 10 \\ 0 & 1\end{array}$

$\stackrel{\infty}{\infty}$
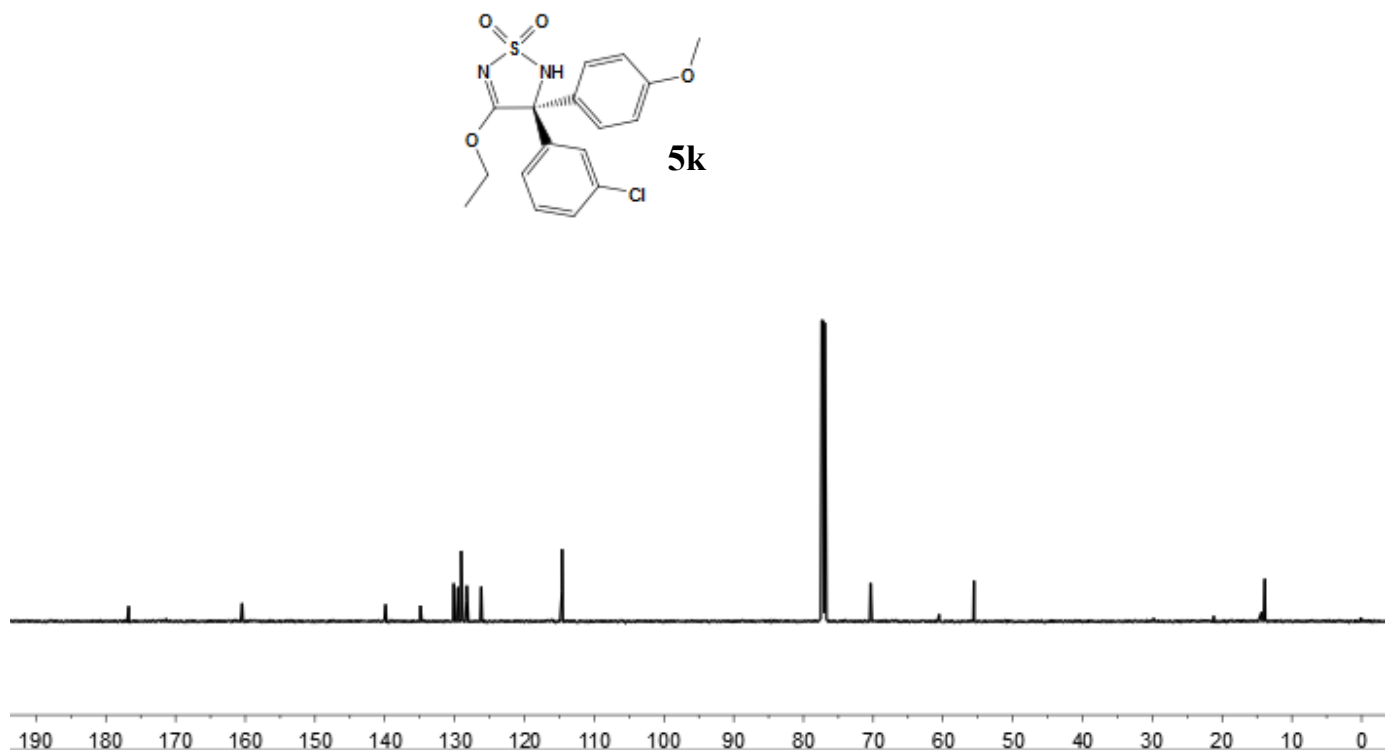

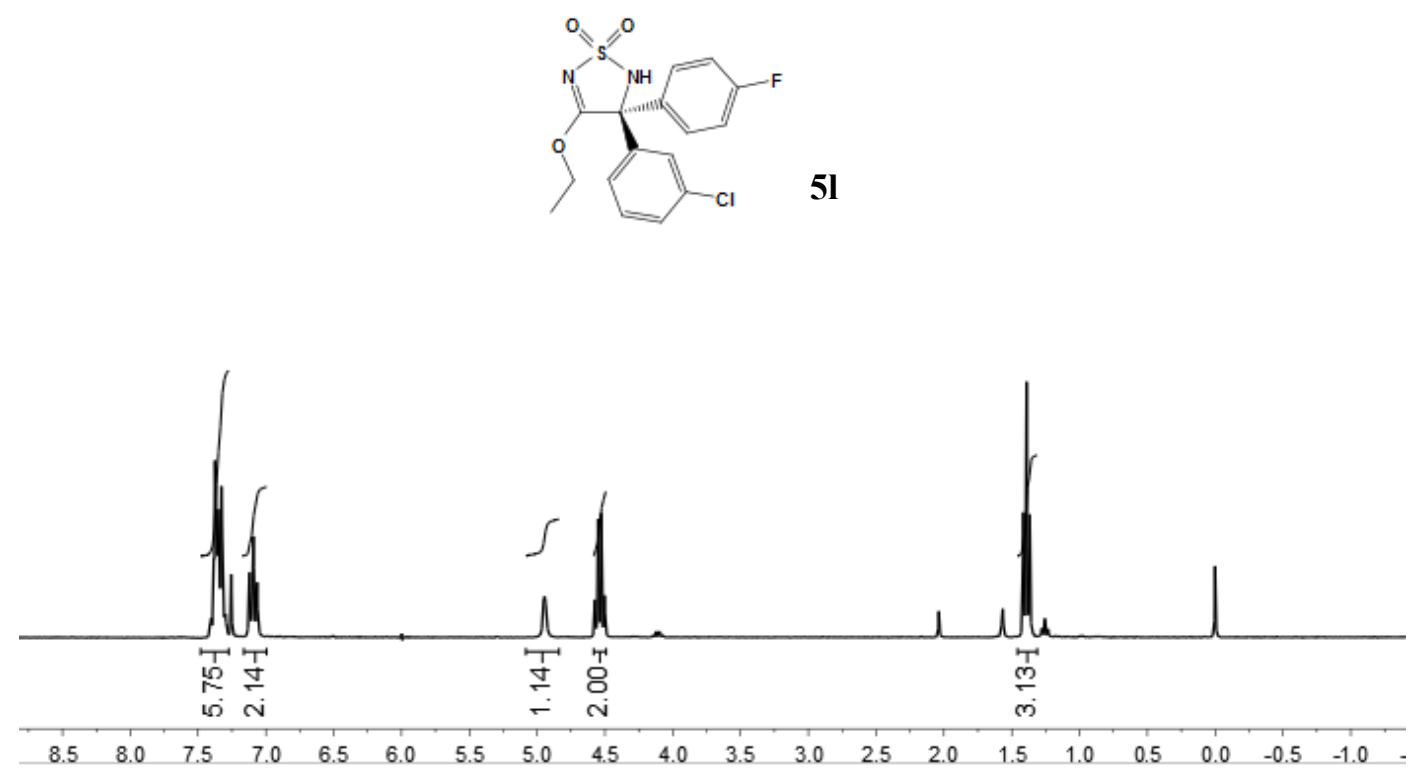

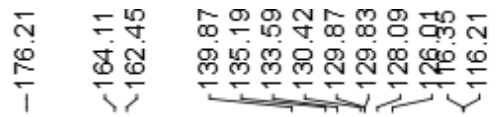

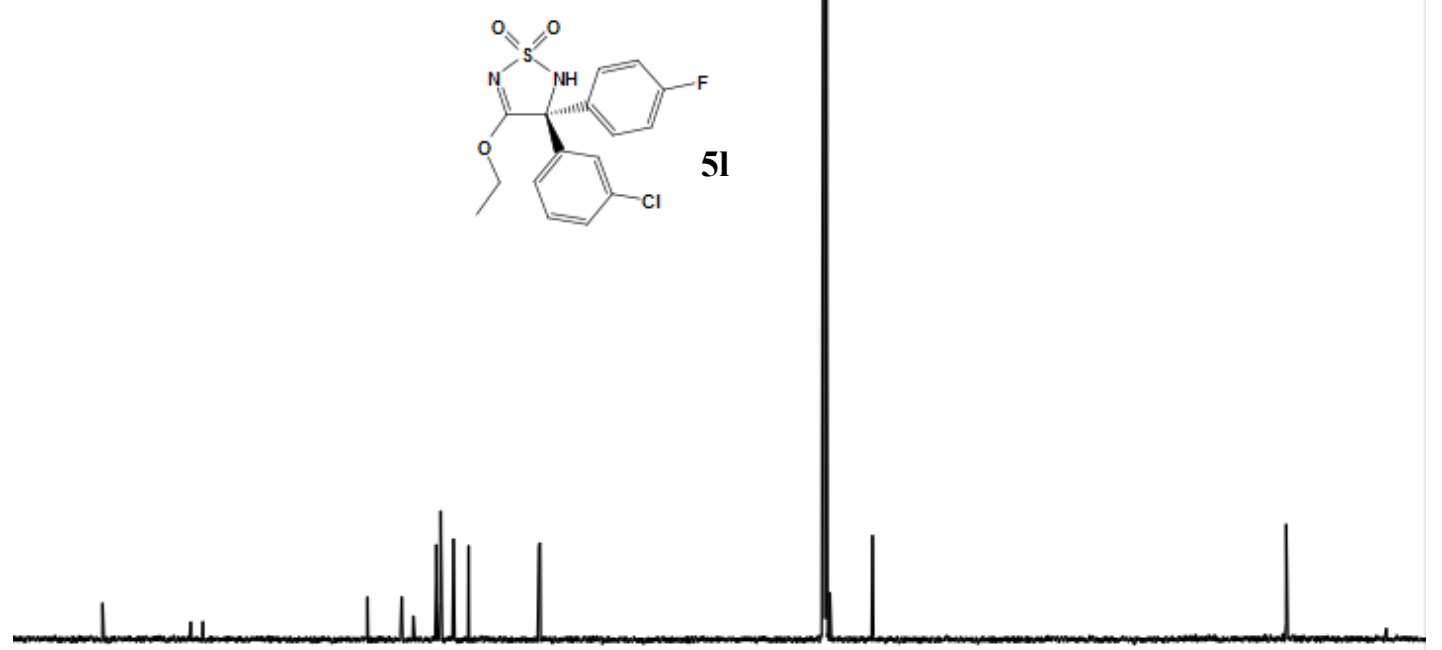

5l

$\underset{1}{\stackrel{\oplus}{\circ}}$

$\stackrel{\infty}{\stackrel{\infty}{m}}$

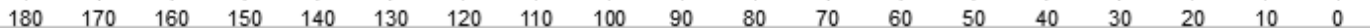




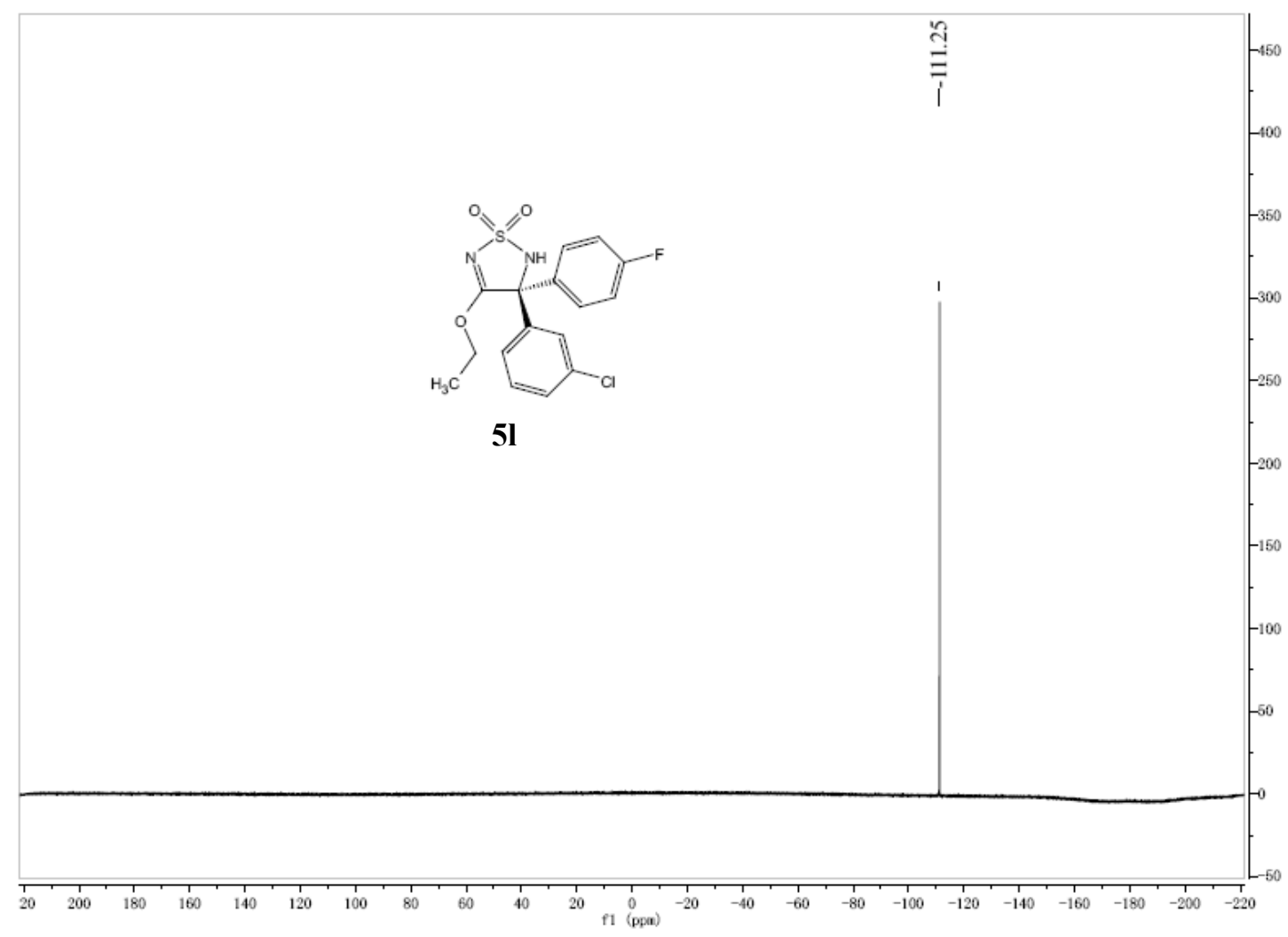

ผ

rmMrnmmrn

অ্

N N N

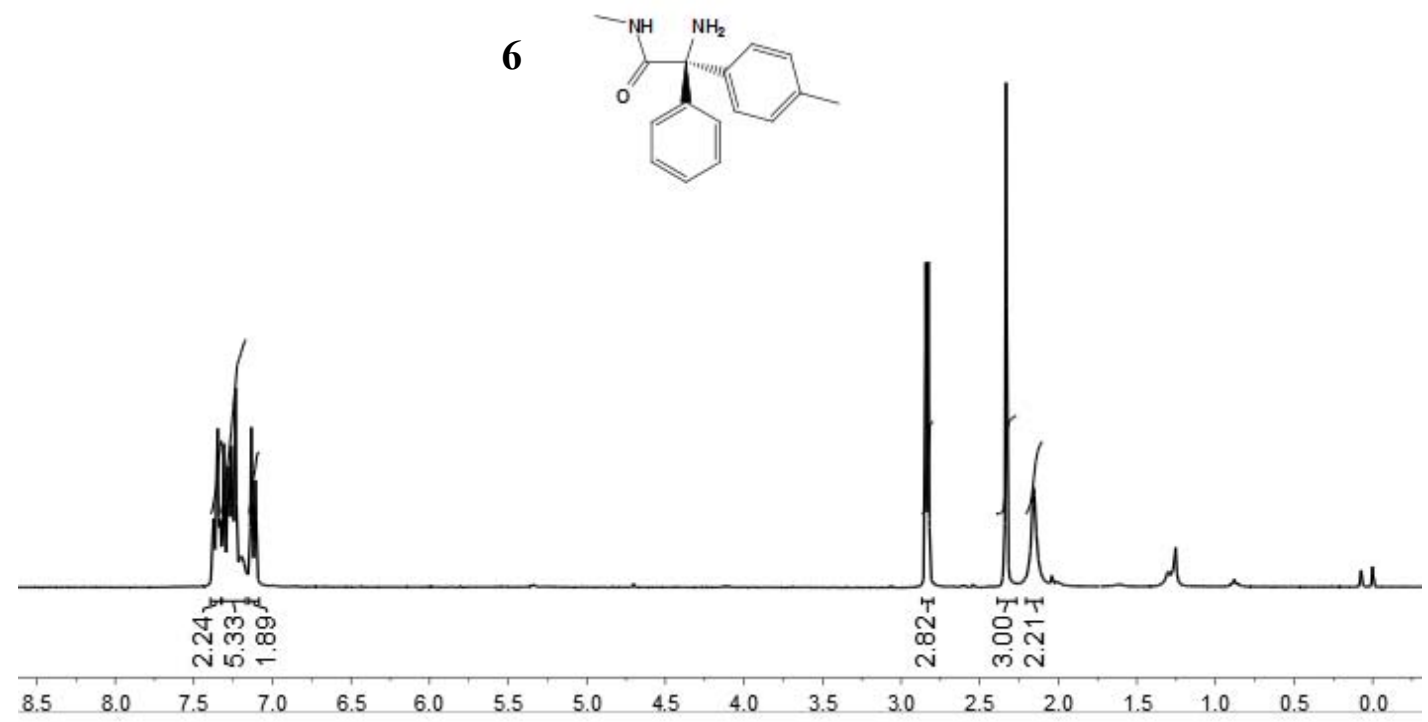




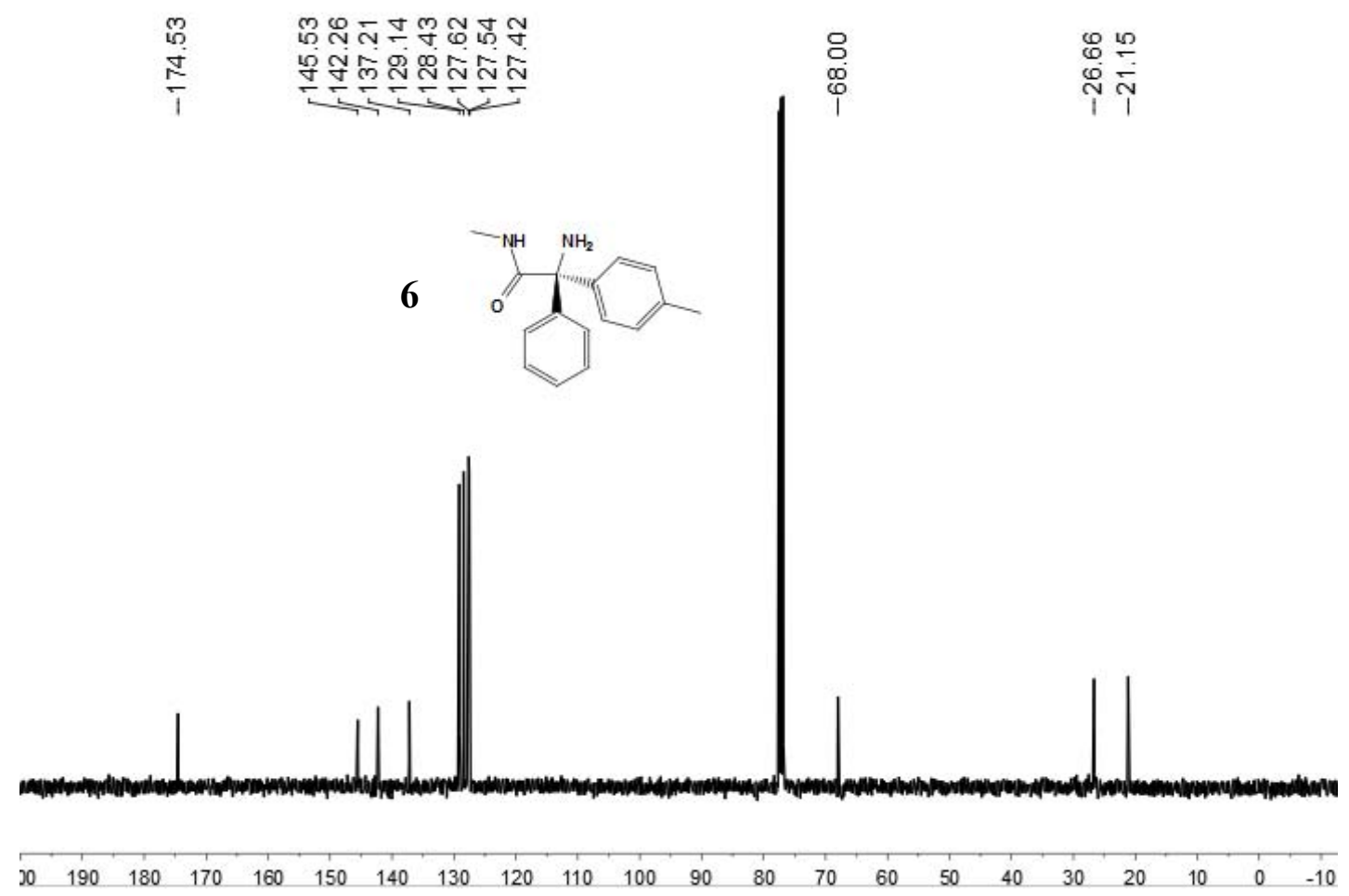

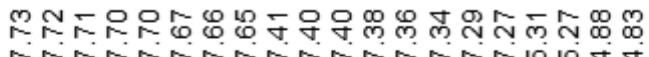

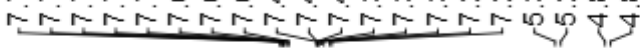

$\frac{1}{p}$

7
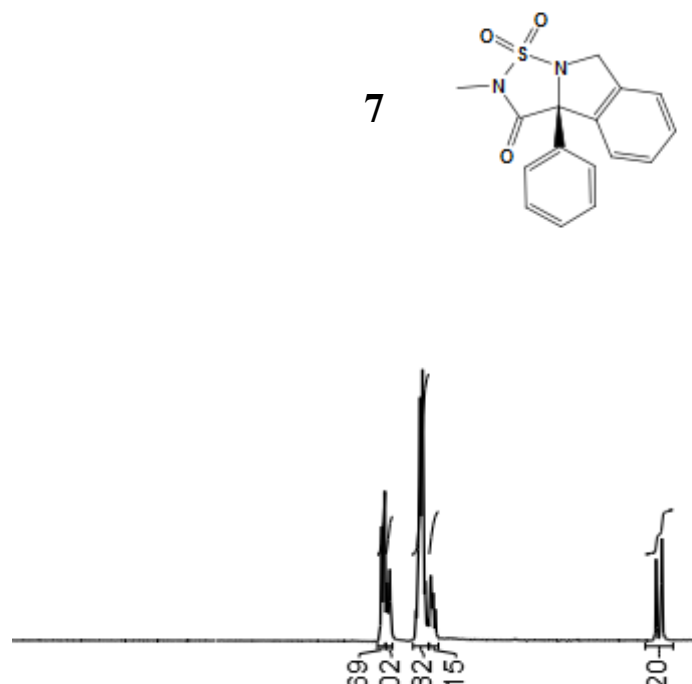

कृष

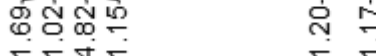

$\begin{array}{lllllllllllllllllllllllll}10.5 & 9.5 & 9.0 & 8.5 & 8.0 & 7.5 & 7.0 & 6.5 & 6.0 & 5.5 & 5.0 & 4.5 & 4.0 & 3.5 & 3.0 & 25 & 2.0 & 1.5 & 1.0 & 0.5 & 0.0 & & -1.0\end{array}$ 


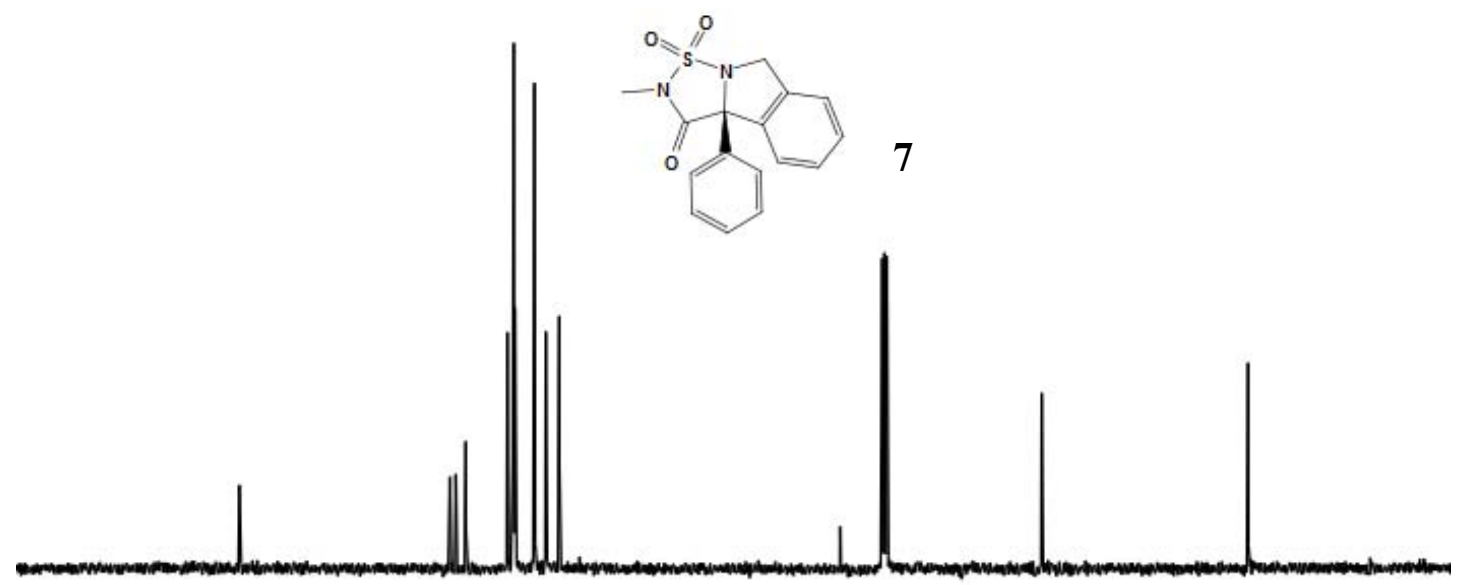

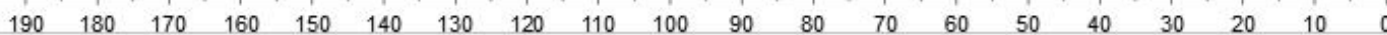
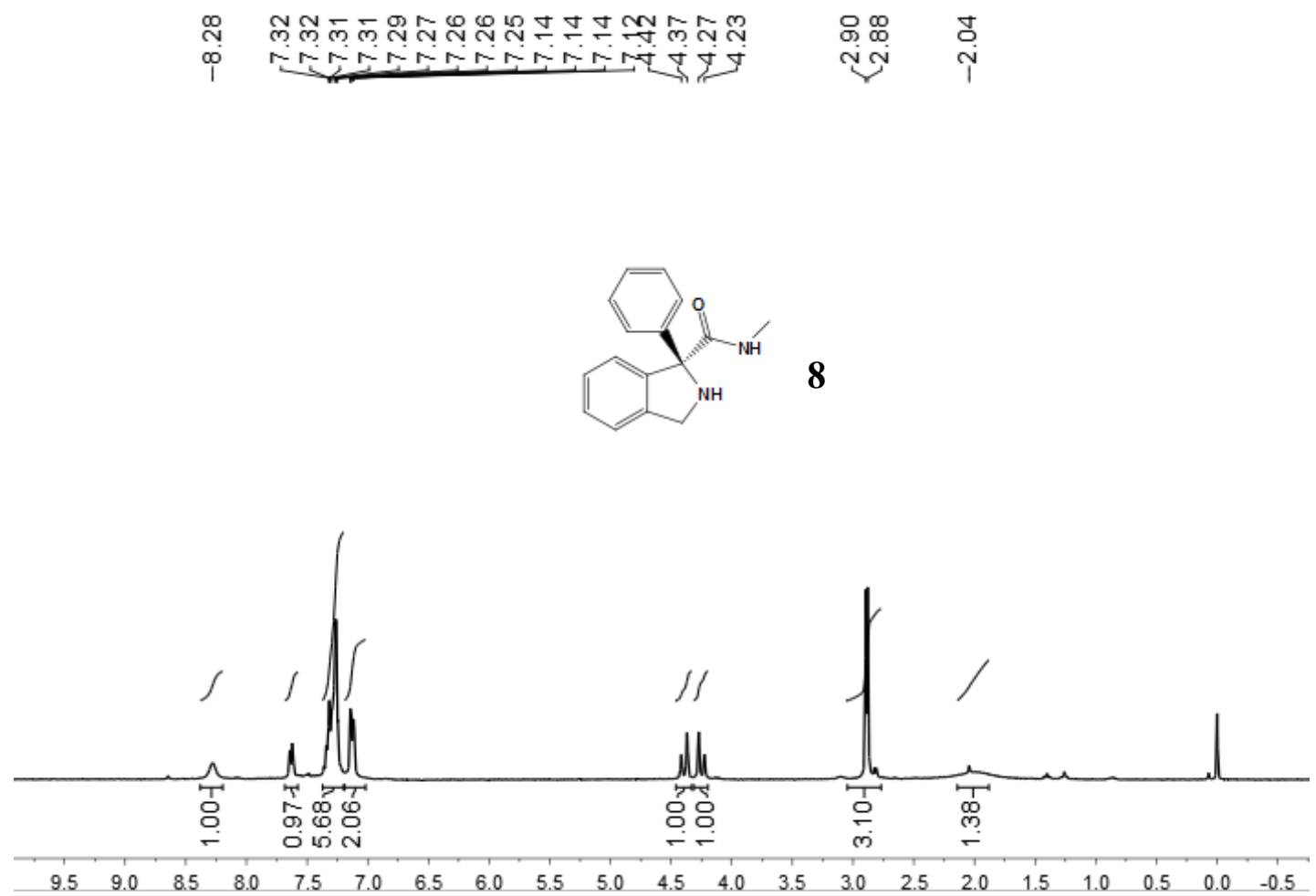


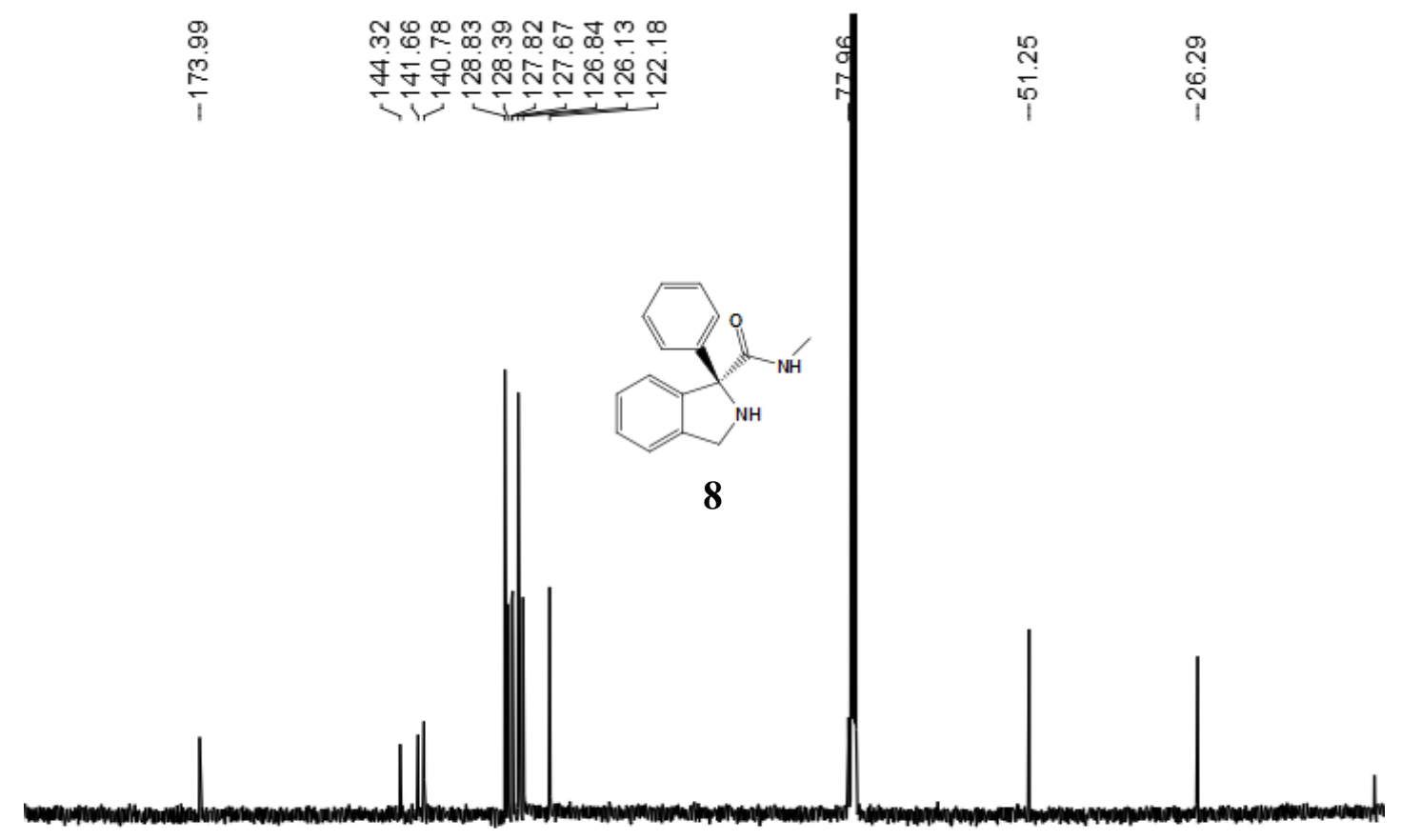

$\begin{array}{lllllllllllllllllllll}10 & 190 & 180 & 170 & 160 & 150 & 140 & 130 & 120 & 110 & 100 & 90 & 80 & 70 & 60 & 50 & 40 & 30 & 20 & 10 & 0\end{array}$

कर

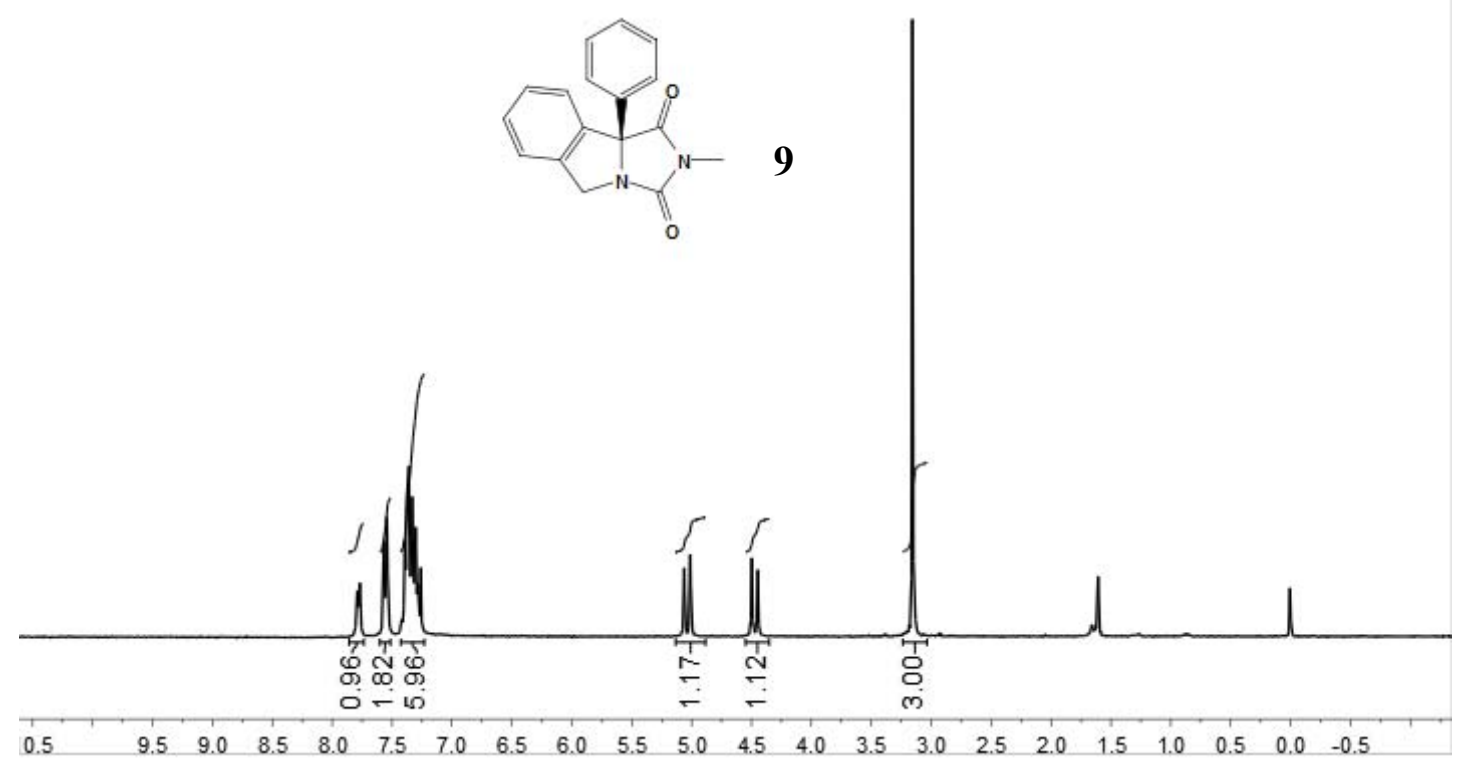




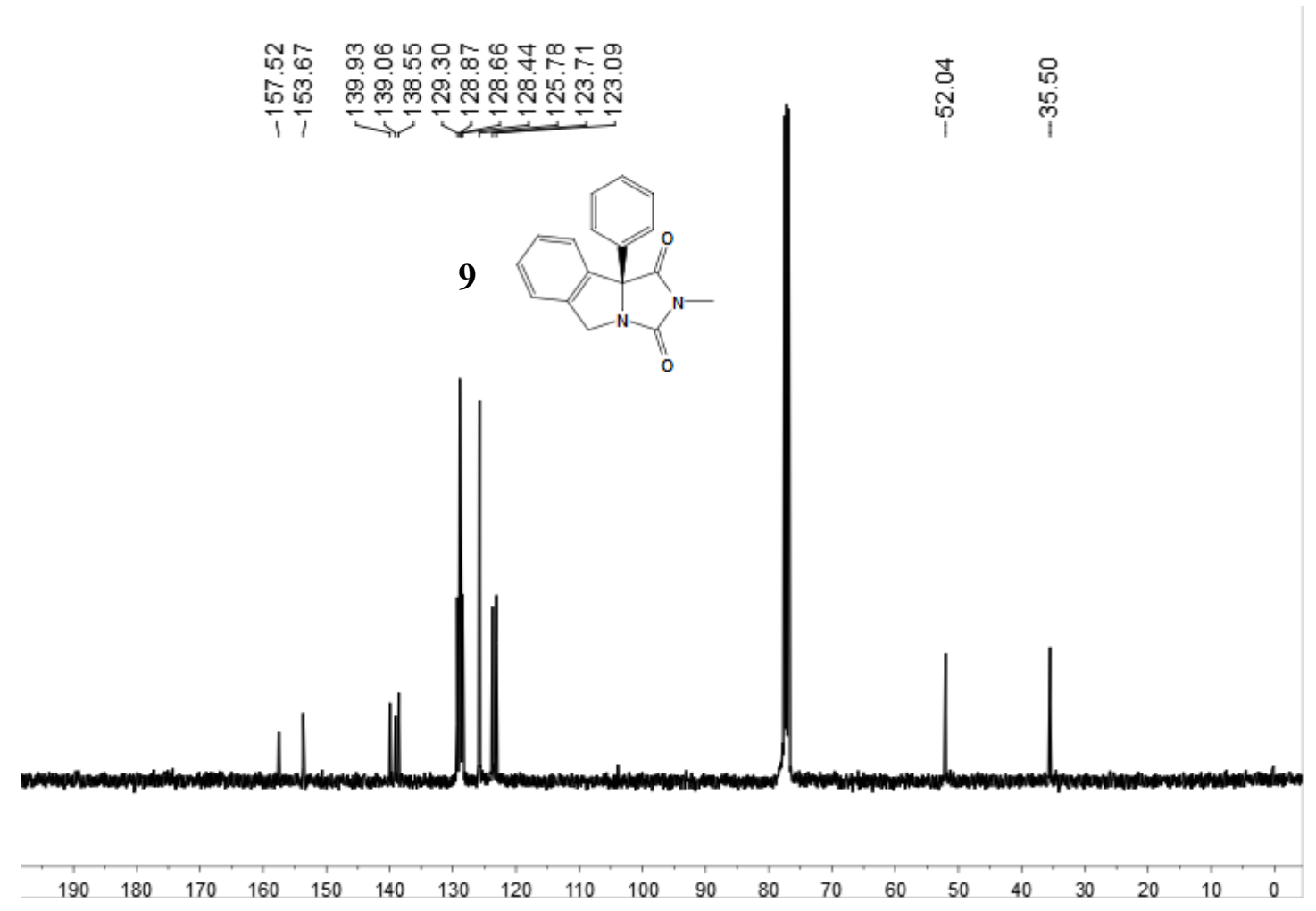

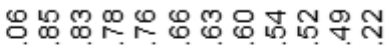

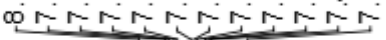

œִ

$\underbrace{\infty}$

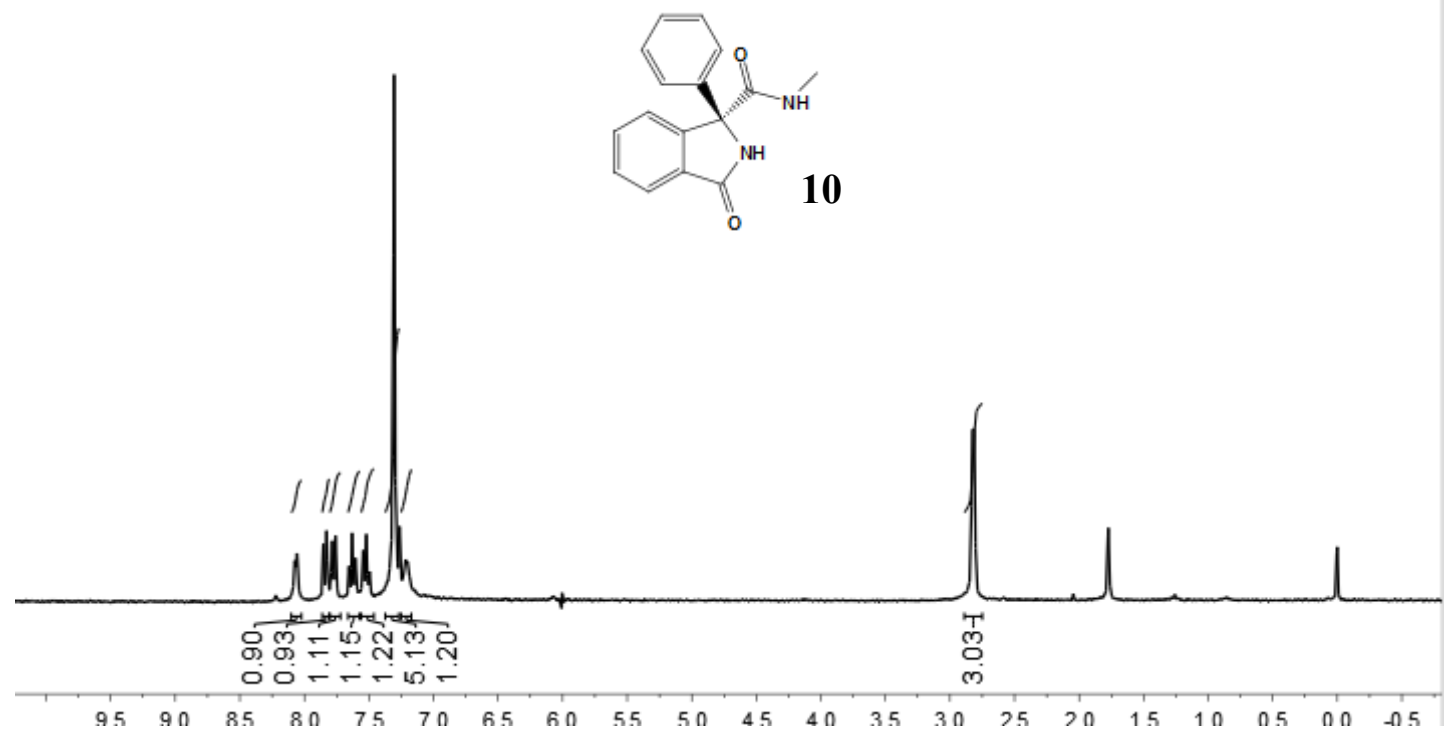




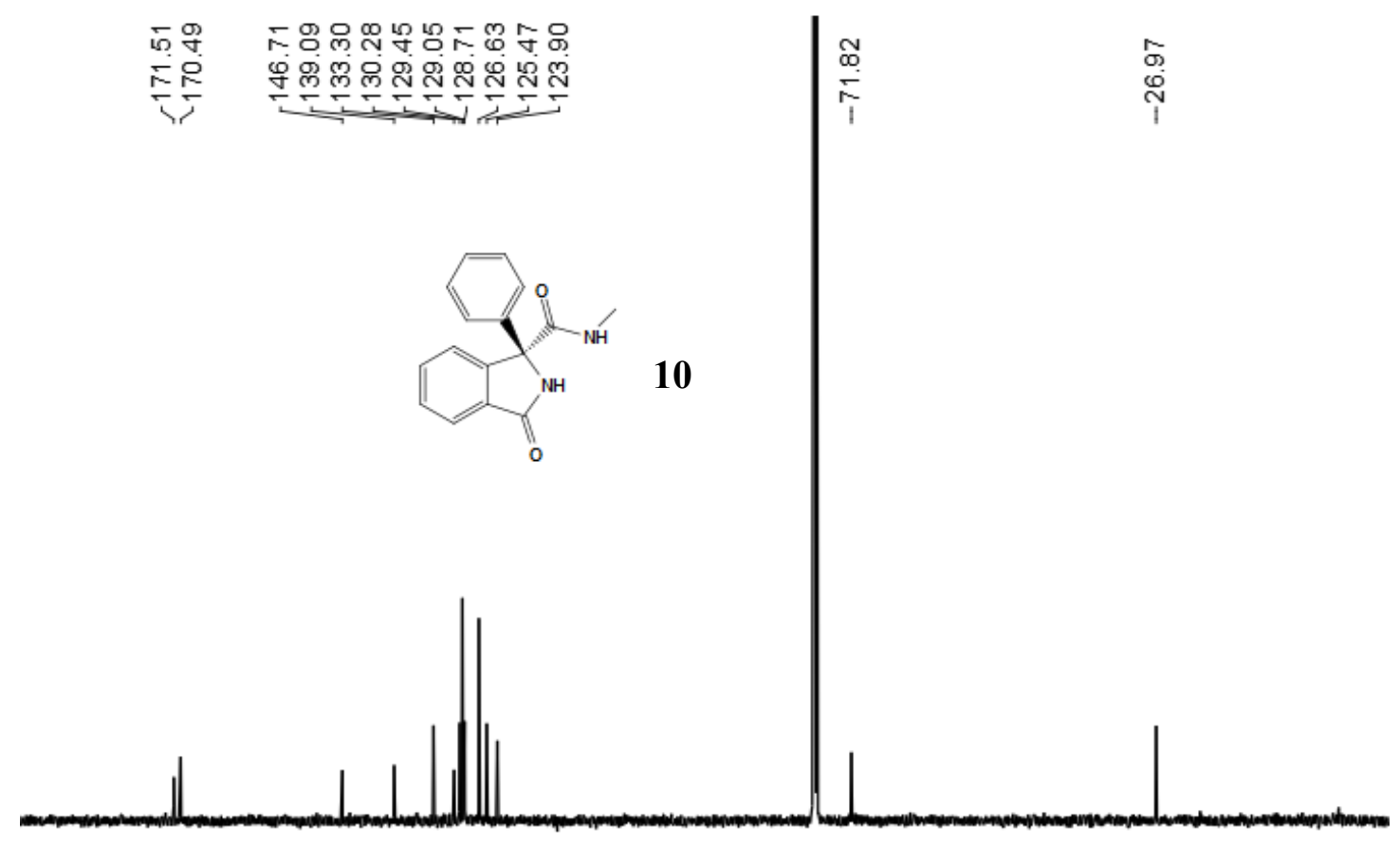

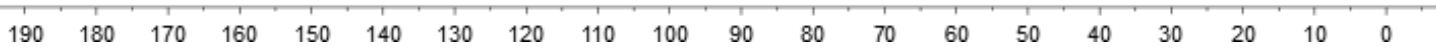

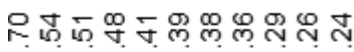

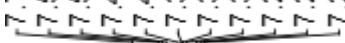
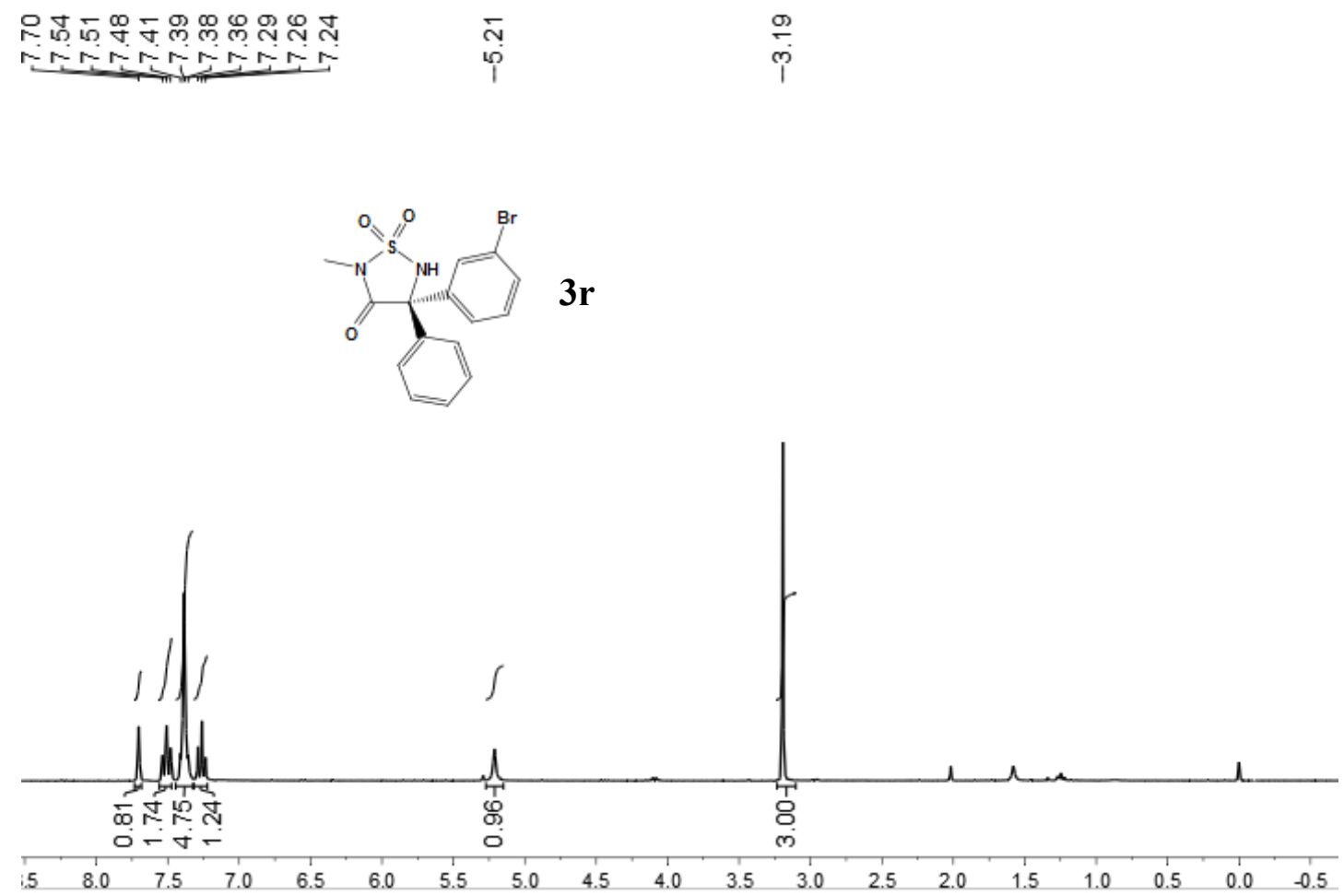


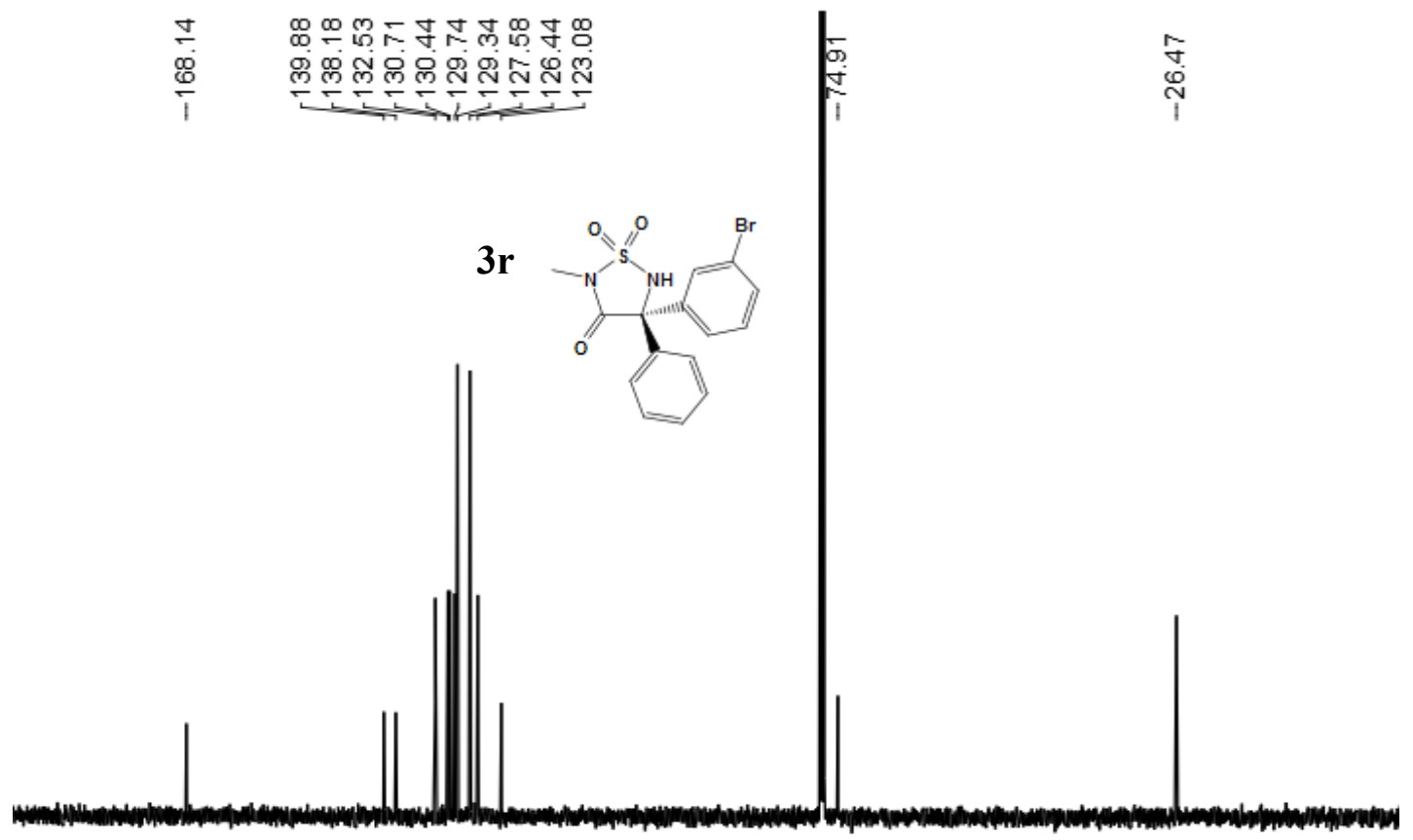

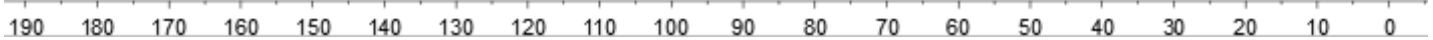

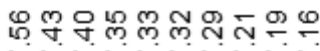

riminiminiminim

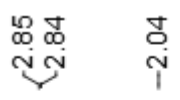

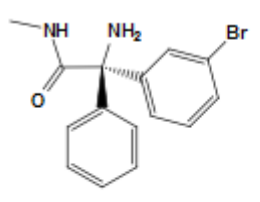

11

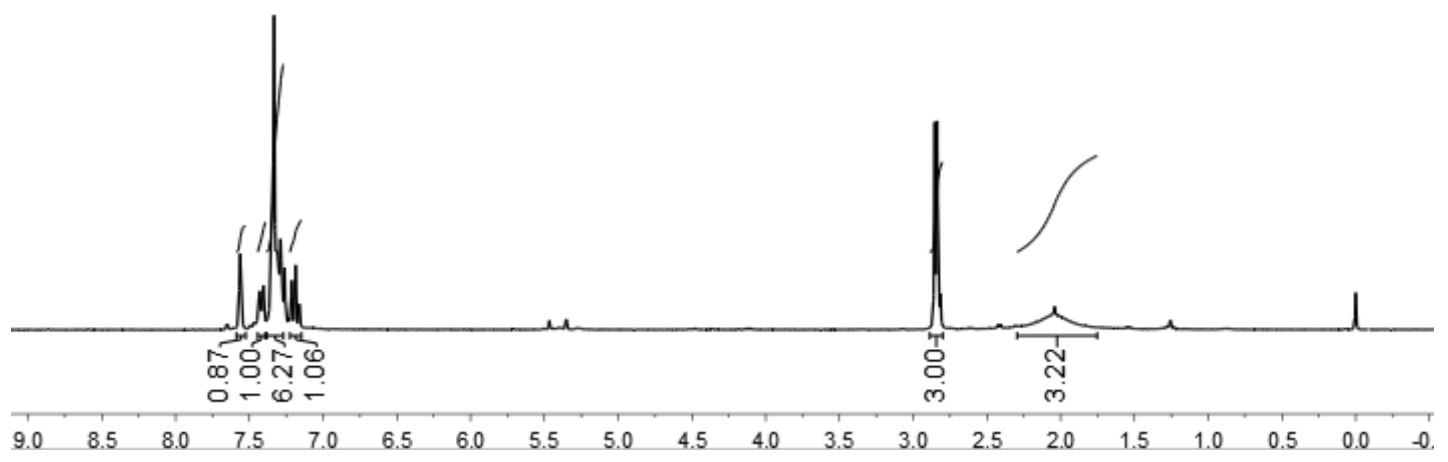




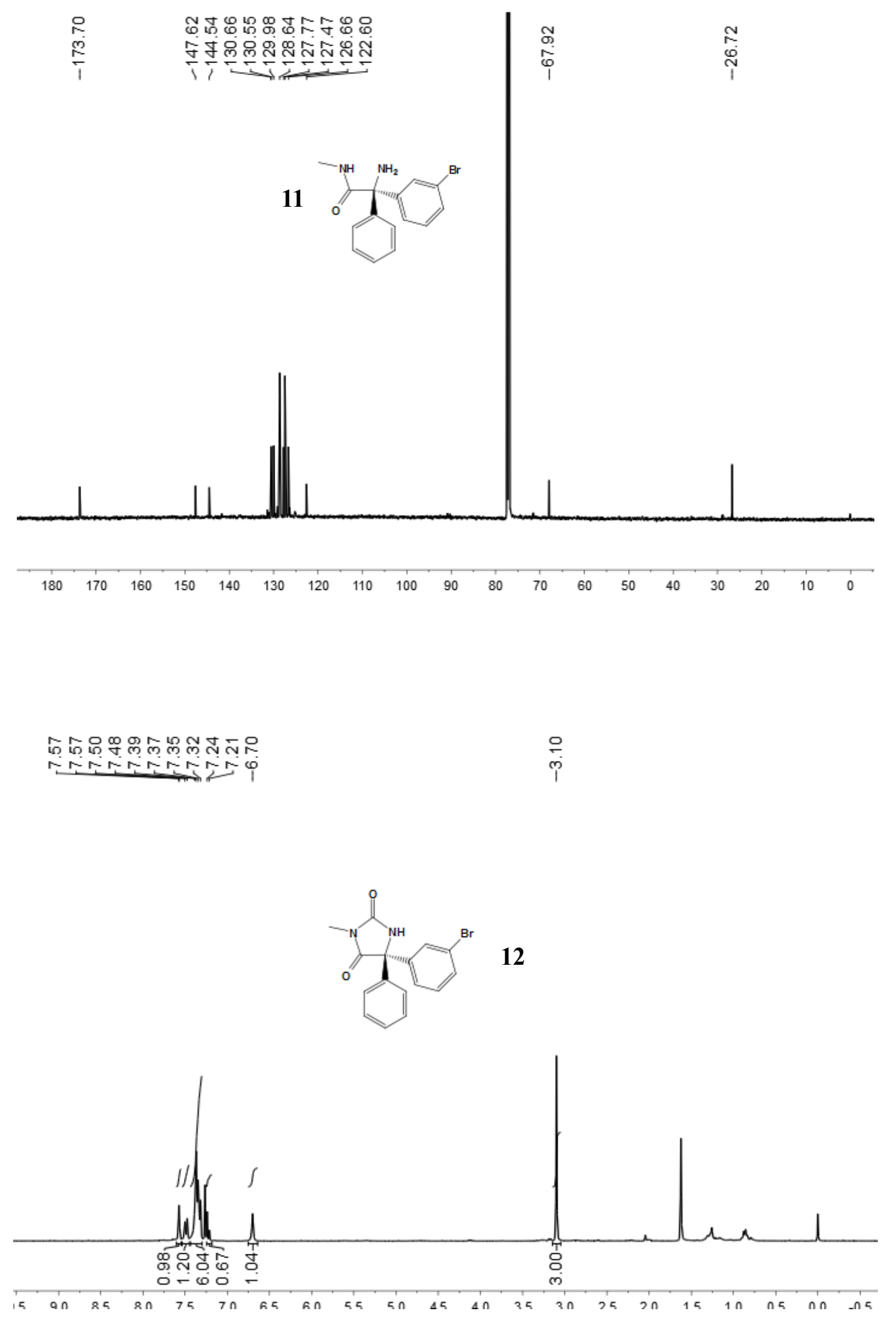




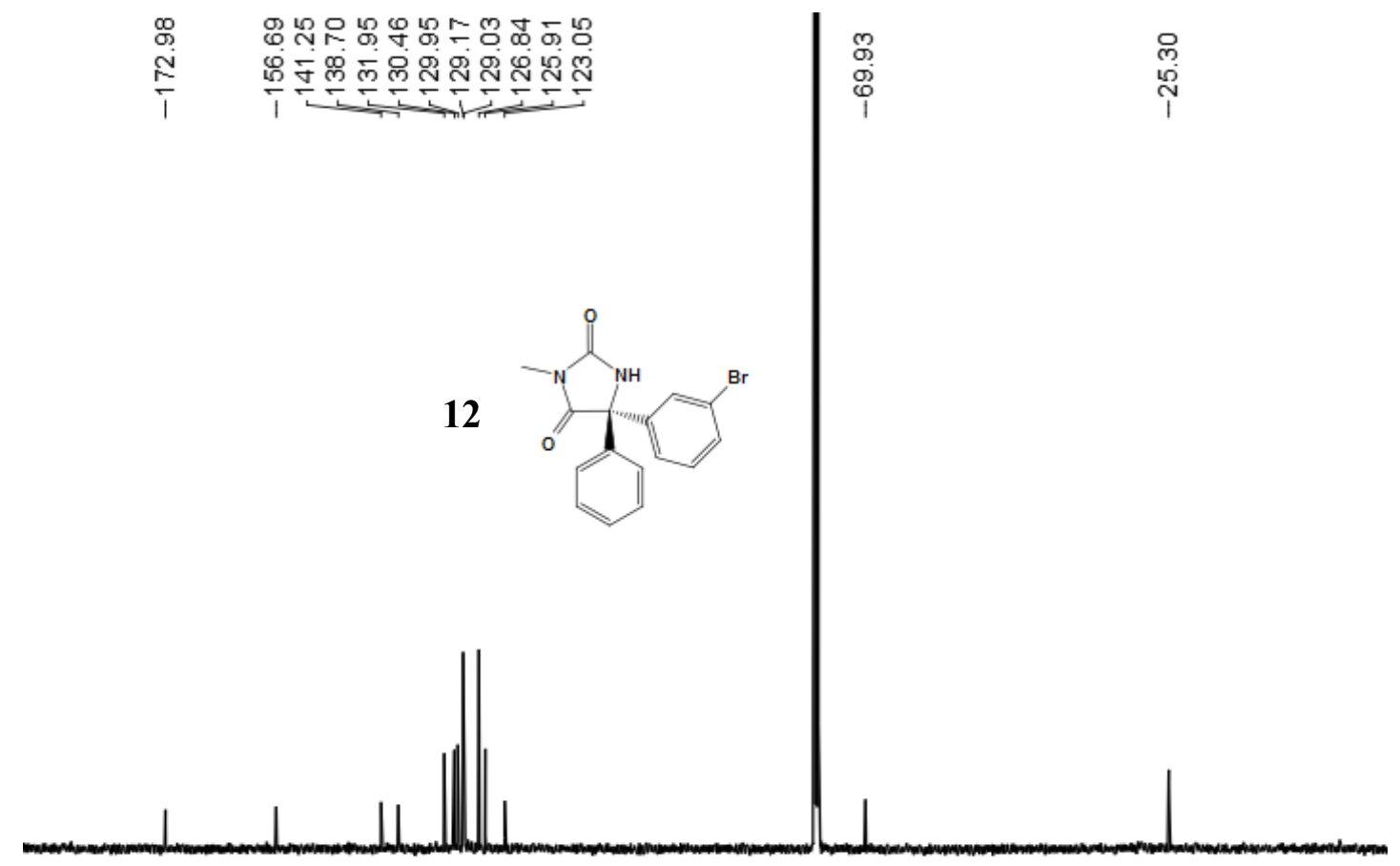

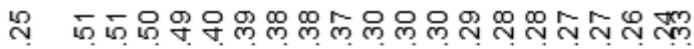

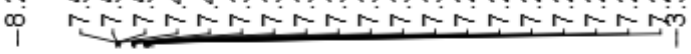

13
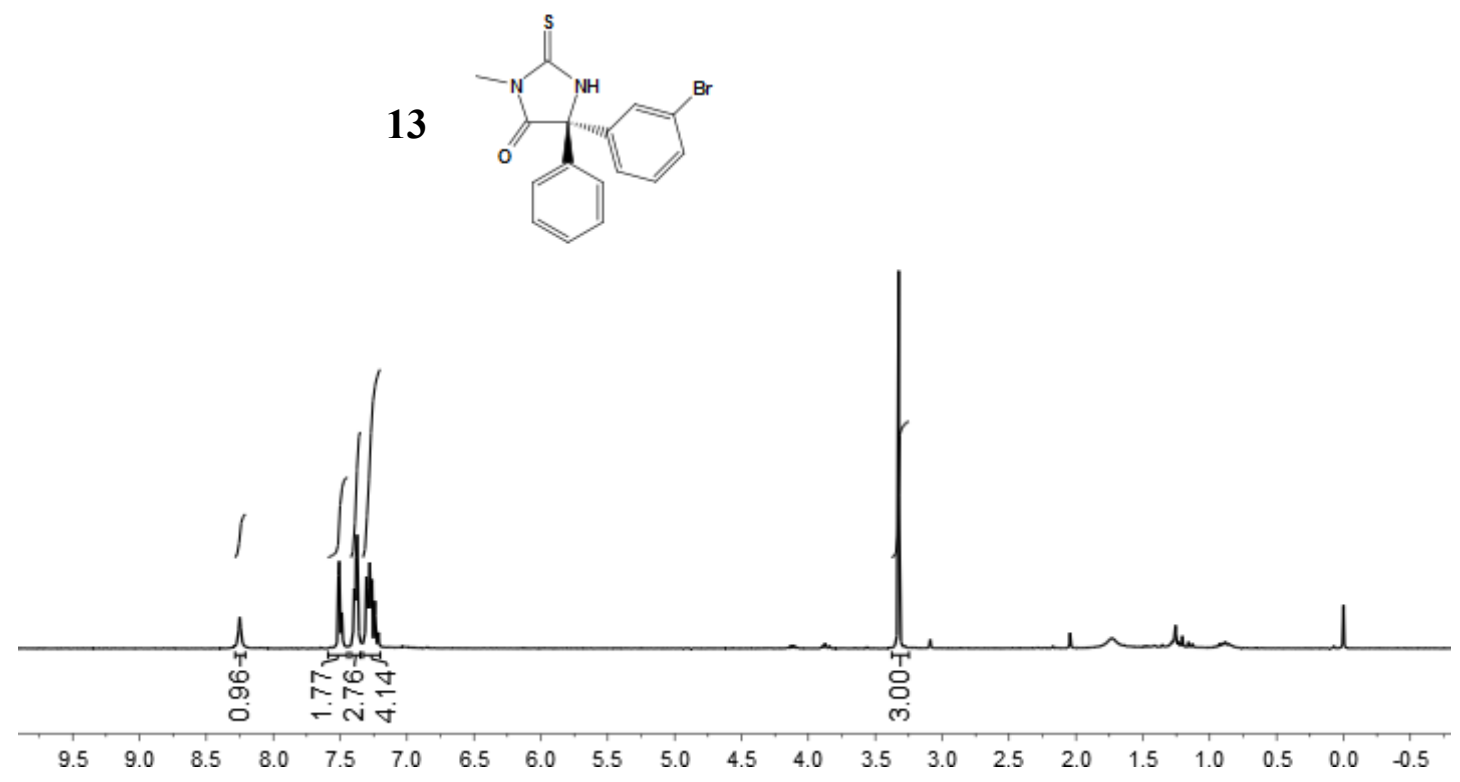


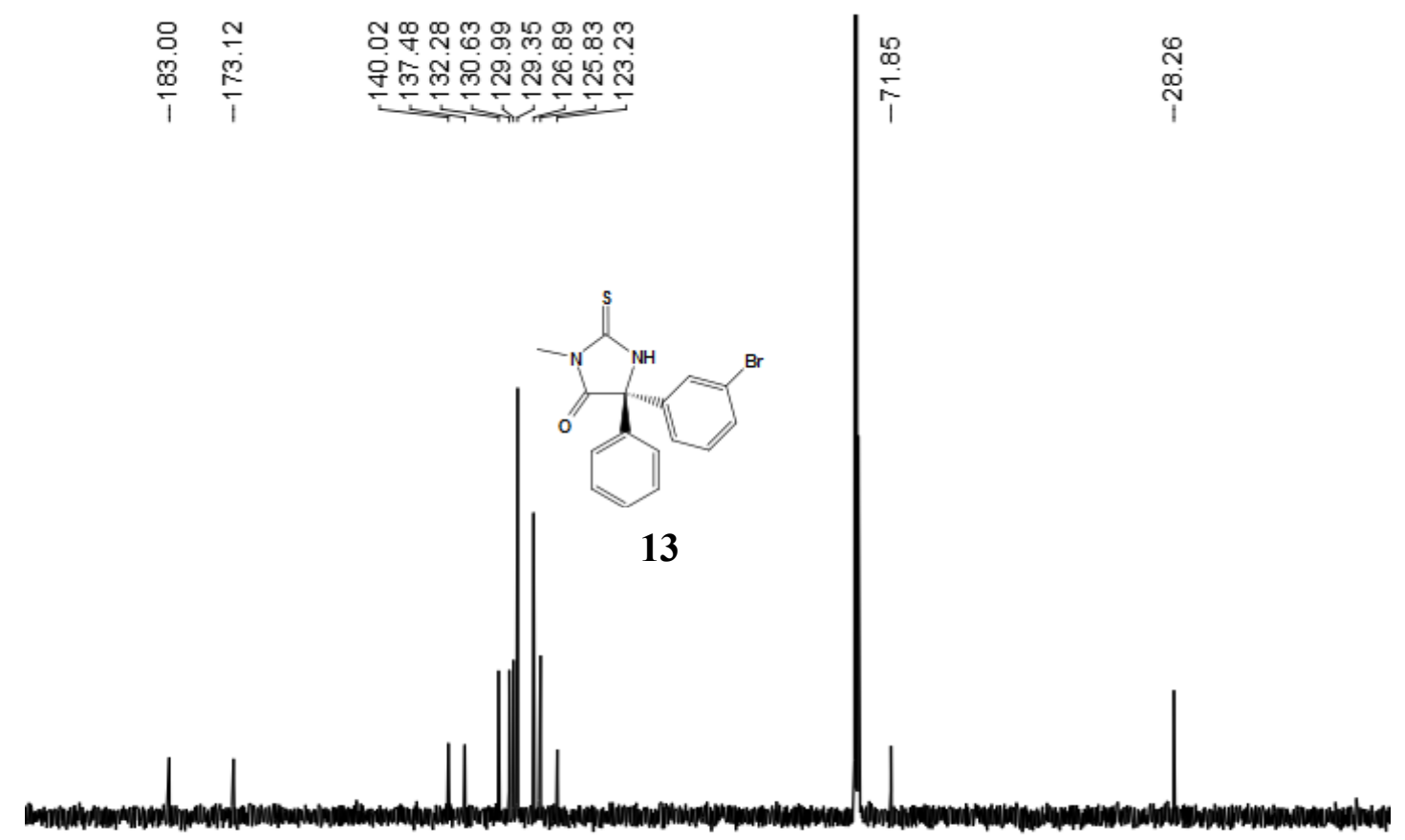

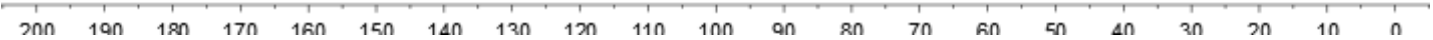

